\title{
WestVirginiaUniversity
}

THE RESEARCH REPOSITORY @ WVU

Graduate Theses, Dissertations, and Problem Reports

2000

\section{Hazardous materials database}

Veena Vadiala

West Virginia University

Follow this and additional works at: https://researchrepository.wvu.edu/etd

\section{Recommended Citation}

Vadiala, Veena, "Hazardous materials database" (2000). Graduate Theses, Dissertations, and Problem Reports. 1091.

https://researchrepository.wvu.edu/etd/1091

This Thesis is protected by copyright and/or related rights. It has been brought to you by the The Research Repository @ WVU with permission from the rights-holder(s). You are free to use this Thesis in any way that is permitted by the copyright and related rights legislation that applies to your use. For other uses you must obtain permission from the rights-holder(s) directly, unless additional rights are indicated by a Creative Commons license in the record and/ or on the work itself. This Thesis has been accepted for inclusion in WVU Graduate Theses, Dissertations, and Problem Reports collection by an authorized administrator of The Research Repository @ WVU. For more information, please contact researchrepository@mail.wvu.edu. 


\title{
HAZARDOUS MATERIALS DATABASE
}

\author{
Veena Vadiala
}

Thesis submitted to the College of Engineering and Mineral Resources at West Virginia University in partial fulfillment of the requirements for the degree of

\author{
Masters of Science \\ in \\ Industrial Engineering
}

\author{
Rashpal S. Ahluwalia, Ph. D., Co-chair \\ David Whaley, Ph. D., Co-chair \\ Robert C. Creese, Ph. D.
}

Department of Industrial and Management Systems Engineering

\author{
Morgantown, West Virginia
}

2000

Keywords: hazardous materials, database, Visual Basic, dismantling 


\section{ABSTRACT \\ HAZARDOUS MATERIALS DATABASE}

\section{Veena Vadiala}

Handling and disposal of a retired object can be a major component of its Life Cycle Cost. Often, during dismantling of a retired object many hazardous materials are released. Disposal of hazardous materials also need to comply with various federal regulations. Agencies like Occupational Safety and Health Administration (OSHA) and the Environmental Protection Agency (EPA) set these safety regulations. It is possible to apply generic exposure and release controls to protect workers from hazardous materials. For the most cost effective hazard controls it is necessary first to identify the materials and their properties of primary concern. There is a need to have an integrated database for properties of Hazardous Materials.

This project developed a database for properties of hazardous materials. The database was implemented in Microsoft Access. Thirty-four chemicals and their categories were identified. These chemicals are encountered during dismantling of a retired object. The database currently contains 60 main fields, which also contain subfields. Information such as its physical properties, chemical properties, health hazards, releases from demolition or various other industrial processes and references to safety, health and environmental regulations can be obtained from this database. A decision support system was developed as a front end to Access. The decision support system was implemented in Visual Basic.

In the future, this database can be expanded to include non-hazardous materials. The database capabilities were demonstrated on the hazardous materials occurring in the ship dismantling industry. It is expected that the database will save significant time and cost in data retrieval. Information retrieval from the database is through an intuitive graphical interface, and suitable for use by a non-computer person. 


\section{Table of Contents}

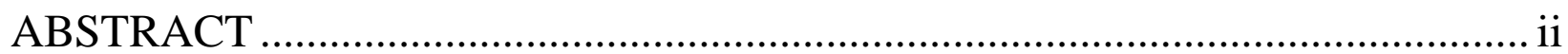

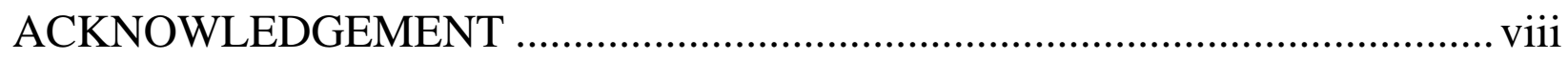

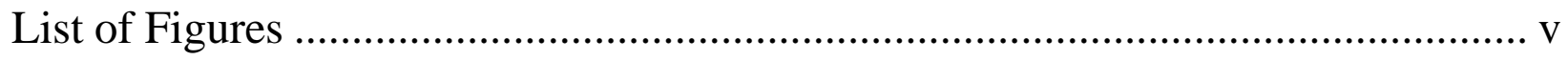

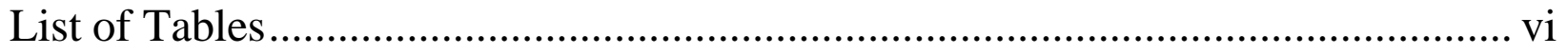

CHAPTER 1: INTRODUCTION ........................................................................... 1

1.1 Databases and Decision Support Systems........................................................................... 1

1.2 Need for an Integrated Database for Hazardous Materials .................................................... 2

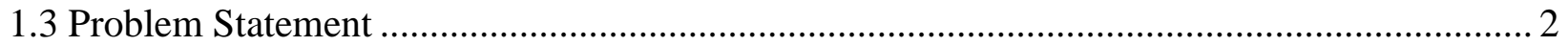

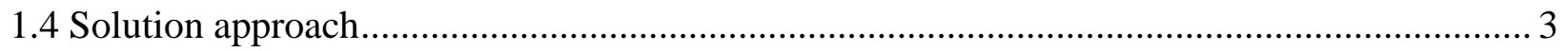

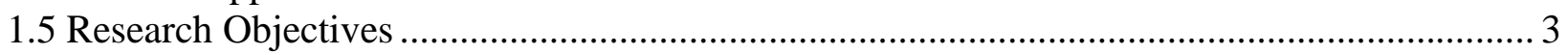

CHAPTER2: LITERATURE REVIEW ............................................................. 5

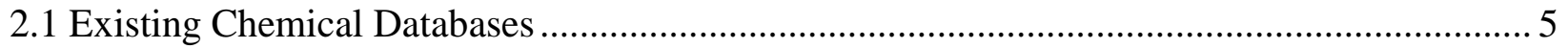

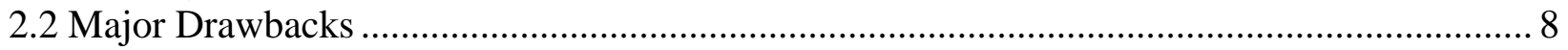

2.3 Sources used to populate the Hazardous Materials Database ……………………................... 9

CHAPTER 3: DATABASE DESIGN............................................................ 11

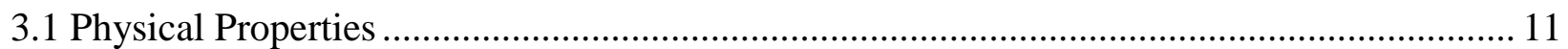

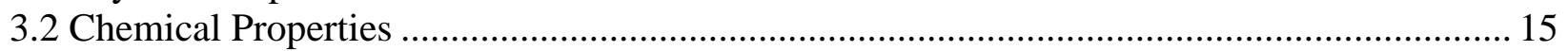

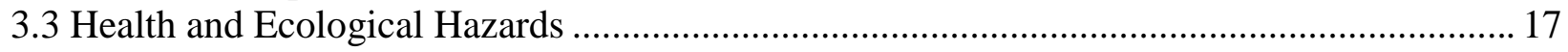

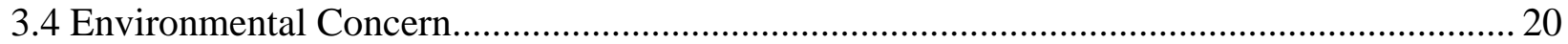

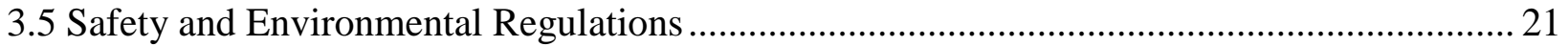

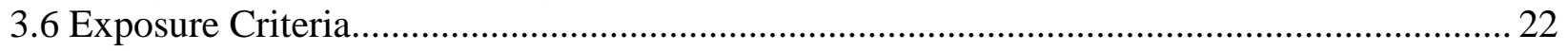

CHAPTER 4: IMPLEMENTATION_........................................................... 25

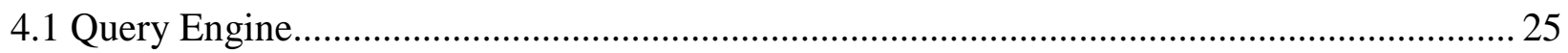

CHAPTER 5: APLLICATION............................................................................ 35

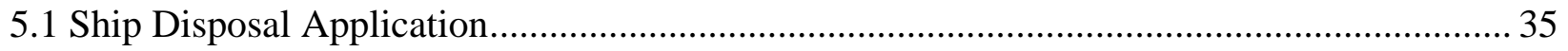

CHAPTER 6: CONCLUSIONS.....................................................................59

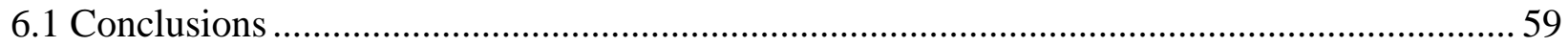

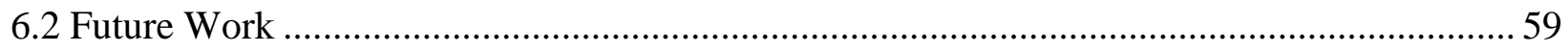

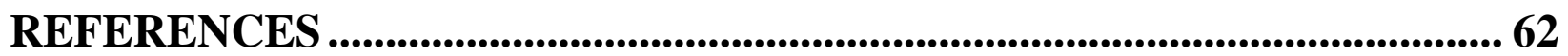




\section{APPENDIX}

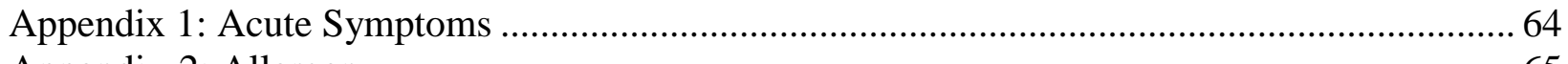

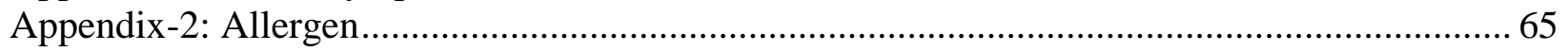

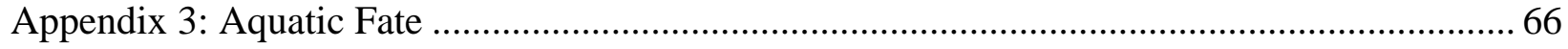

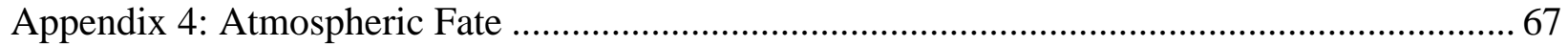

Appendix 5: BOD

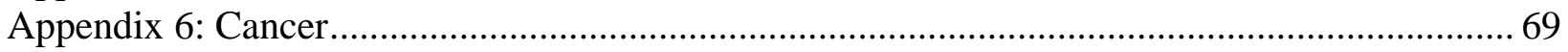

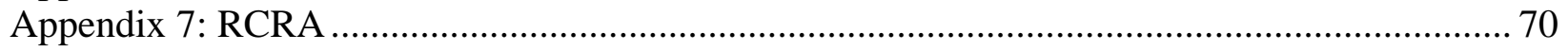

Appendix 8: Chronic Symptoms ................................................................................... 71

Appendix 9: Clean Air Act................................................................................................ 72

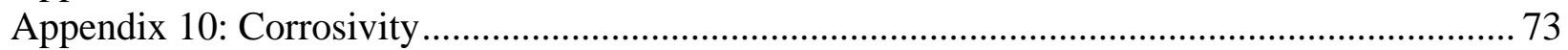

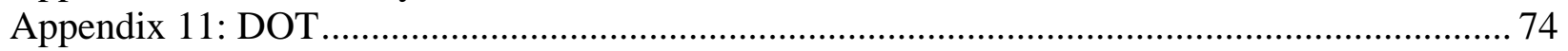

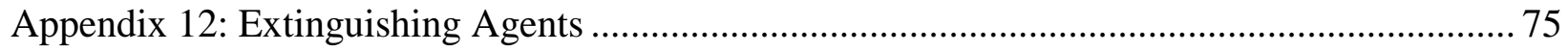

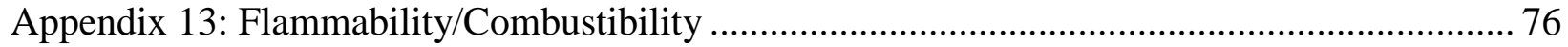

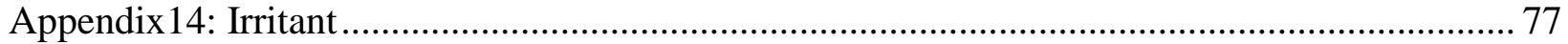

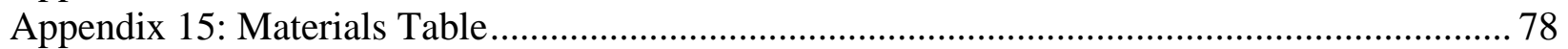

Appendix 16: Oral Acute Aquatic Toxicity ......................................................................... 79

Appendix 17: Percent Dissociated …………………….................................................... 80

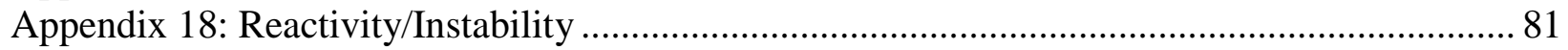

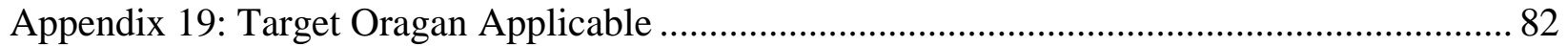

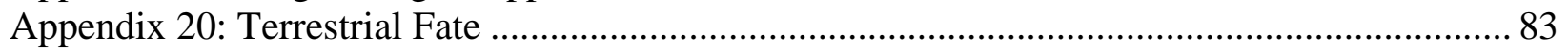

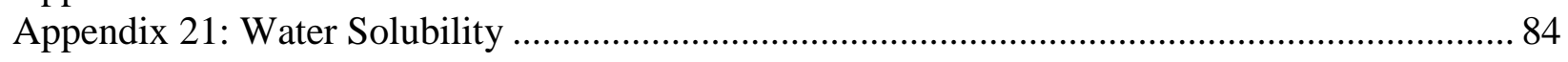

Appendix 22: Worker Exposure Criteria (Chronic-TWA) ………............................................. 85

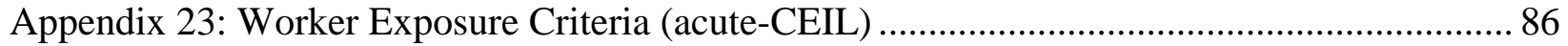

Appendix 24: Worker Exposure Criteria (acute-STEL) …………............................................. 87

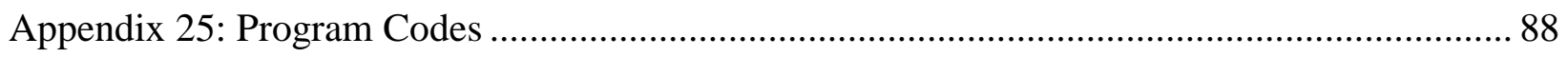

Appendix 25.1: Acute Symptoms ...................................................................................... 88

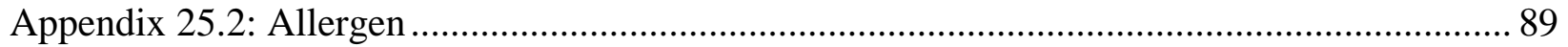

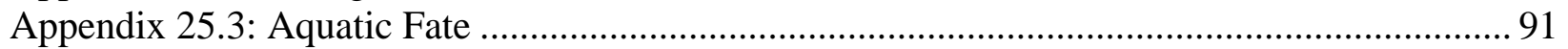

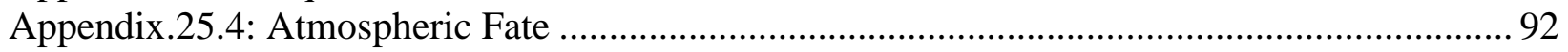

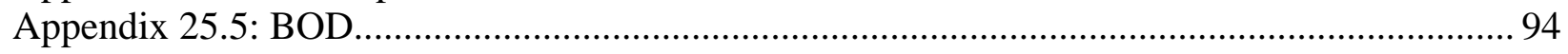

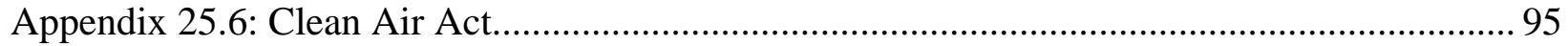

Appendix 25.7: Cancer.......................................................................................................... 97

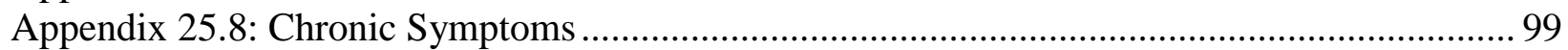

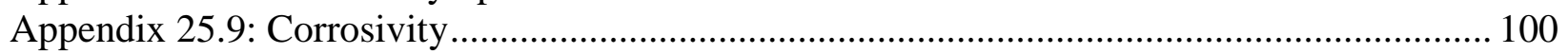

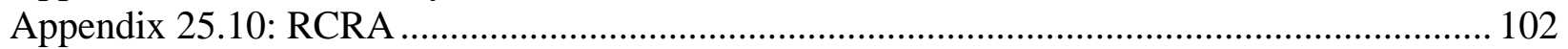

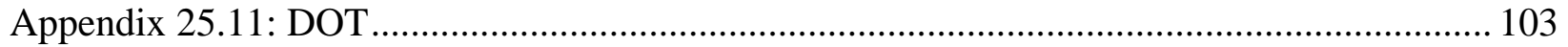

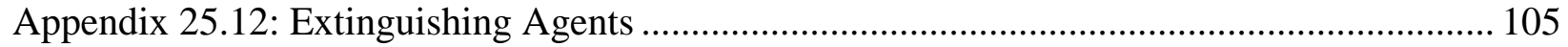

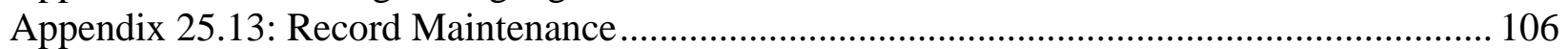

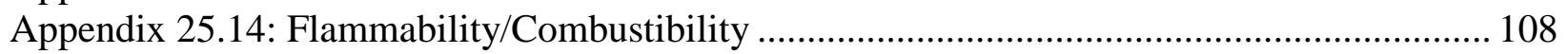

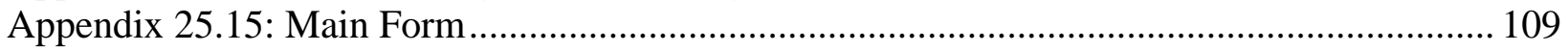

Appendix 25.16: Materials.......................................................................................... 112

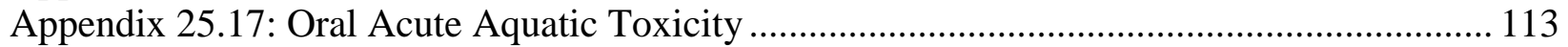

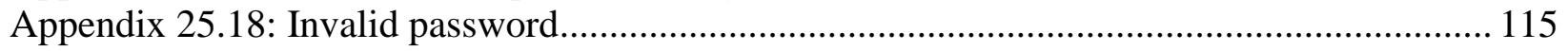

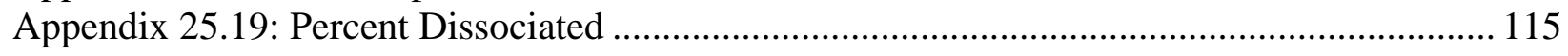




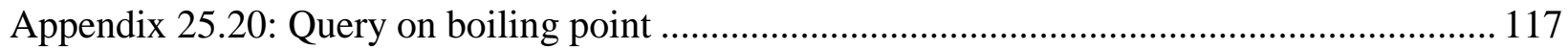

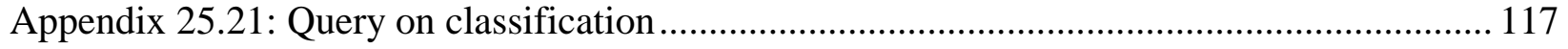

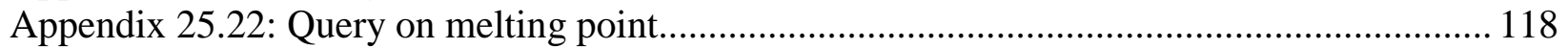

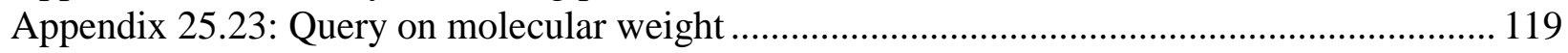

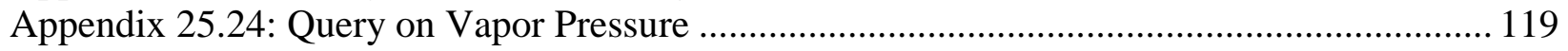

Appendix 25.25: Reactivity/Instability ……………....................................................... 120

Appendix 25.26: Search by Chemical Name ....................................................................... 121

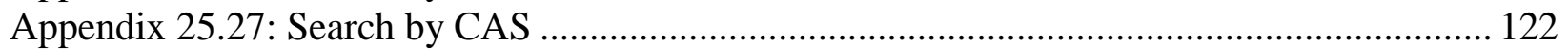

Appendix 25.28: Search by Molecular Formula .................................................................. 123

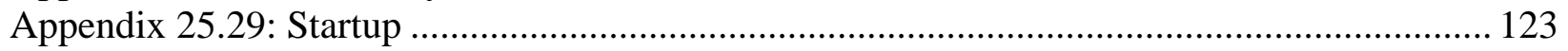

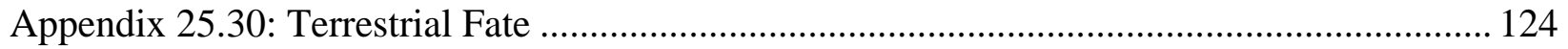

Appendix 25.31: Water Solubility ....................................................................................... 125

Appendix 25.32: Worker Exposure Criteria (acute-CEIL............................................................. 127

Appendix 25.33: Worker Exposure Criteria (acute-STEL...................................................... 128

Appendix 25.34: Worker Exposure Criteria (chronic-TWA ....................................................... 130

Appendix 26: Relationships Diagram of the Tables ............................................................ 132

\section{LIST OF FIGURES}

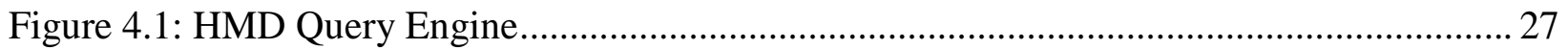

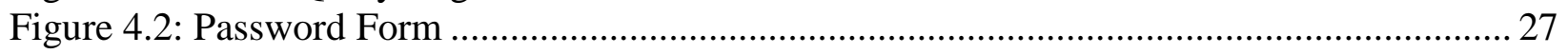

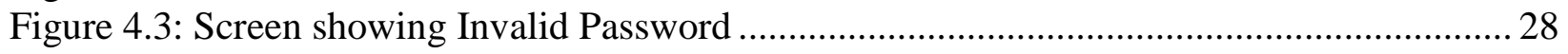

Figure 4.4: Showing Pull Down Menu of File ........................................................................... 28

Figure 4.5: Showing Pull Down Menu of Record Maintenance …….......................................... 29

Figure 4.6: Listing the items in pull down menu for Physical Properties................................... 29

Figure 4.7: Showing Pull Down Menu for chemical Properties ................................................. 30

Figure 4.8: Showing Pull Down Menu for Health Hazards ........................................................... 31

Figure 4.9: Showing the Pull Down Menu for Safety and Environmental Regulations ................ 31

Figure 4.10: Showing the Pull Down Menu for Exposure Criteria.............................................. 32

Figure 4.11: Showing the Pull down Menu for Environmental Concern....................................... 33

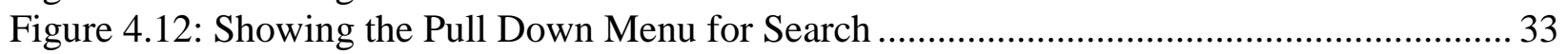

Figure 4.13: Showing the Pull Down Menu for Query .................................................................. 34

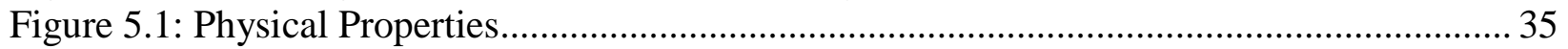

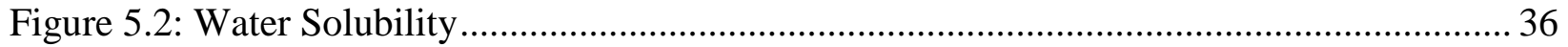

Figure 5.3: Percent Dissociated Table of Physical Properties ....................................................... 36

Figure 5.4: Showing Flammability/Comustibility Table ............................................................ 37

Figure 5.5: Extinguishing Agents Table of Chemical Properties.................................................. 37

Figure 5.6: Reactivity/Instability Table of Chemical Properties................................................... 38

Figure 5.7: Corrosivity Table of Chemical Properties ............................................................. 38

Figure 5.8: Cancer Table of Health and Ecological Hazards .......................................................... 39

Figure 5.9: Showing Aquatic Fate Table ...................................................................................... 39

Figure 5.10: Acute Symptoms Table of Health and Ecological Hazards...................................... 40

Figure 5.11: Chronic Symptoms Table of Health and Ecological Hazards ................................... 40

Figure 5.12: Allergen Table of Health and Ecological Hazards .................................................. 41

Figure 5.13: Oral Acute aquatic Toxicity Table ………......................................................... 41

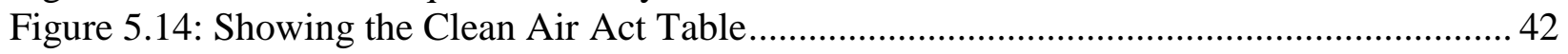

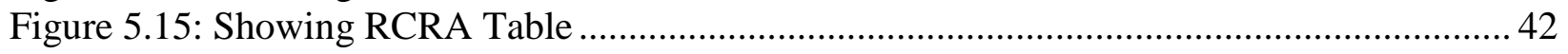


Figure 5.16: DOT Table of Safety and Environmental Regulations..................................... 43

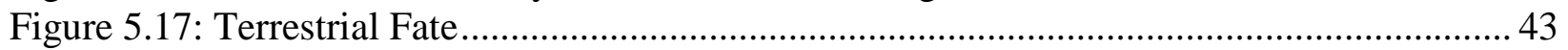

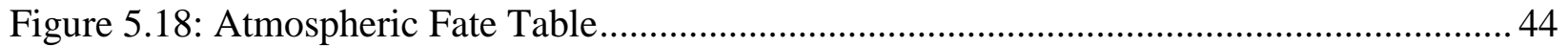

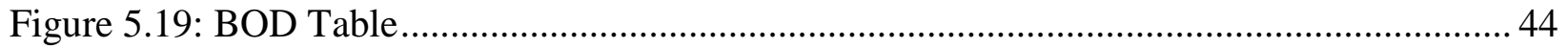

Figure 5.20: Worker Exposure Criteria (acute-STEL) Table .............................................. 45

Figure 5.21: Worker Exposure Criteria (acute-CEIL) Table ................................................. 45

Figure 5.22: Worker Exposure Criteria (chronic-TWA) Table ................................................ 46

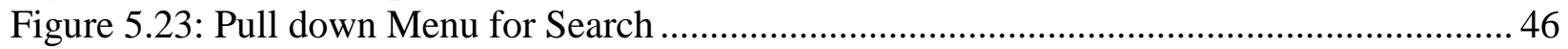

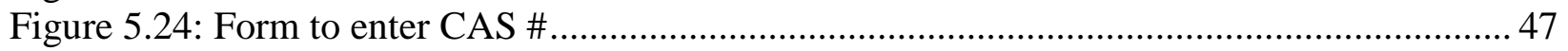

Figure 5.25: Chemical Name Properties ........................................................................ 48

Figure 5.26: Search by Chemical Name........................................................................... 48

Figure 5.27: Form to Search by Molecular Formula............................................................. 49

Figure 5.28: Search by Molecular Formula ..................................................................... 49

Figure 5.29: Search Results Form ................................................................................. 50

Figure 5.30: Pull Down Menu......................................................................................... 51

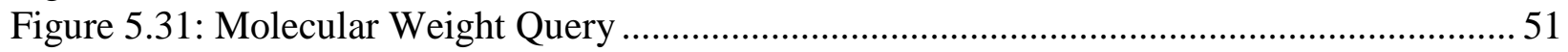

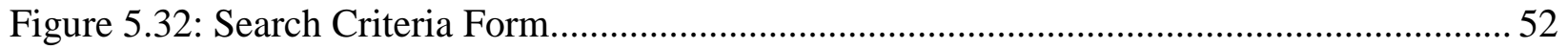

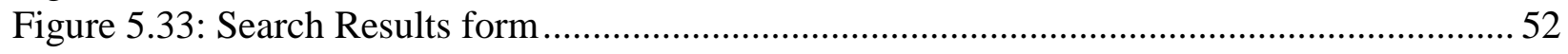

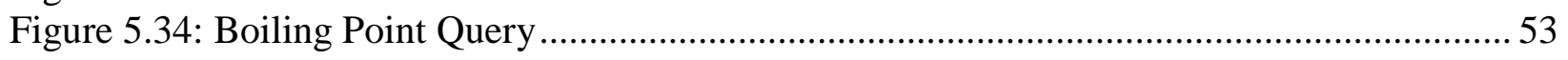

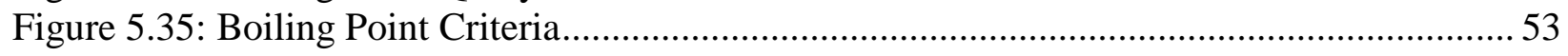

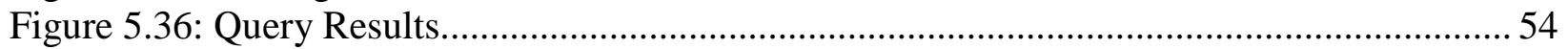

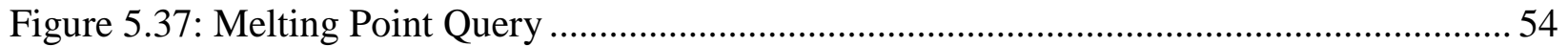

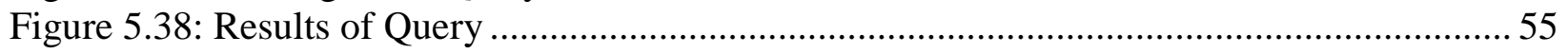

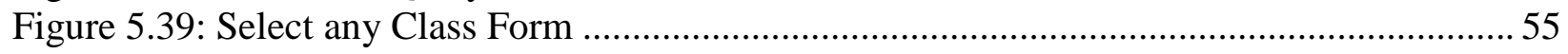

Figure 5.40: Dropdown List of Classes of Compounds ....................................................... 56

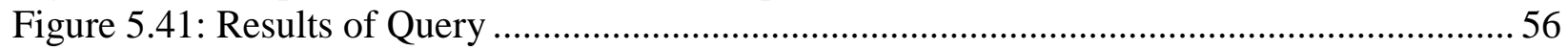

Figure 5.42: Vapor Pressure Query ............................................................................... 57

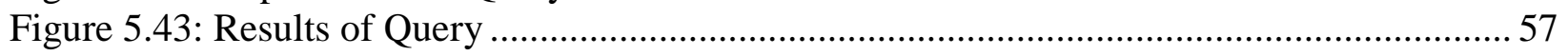

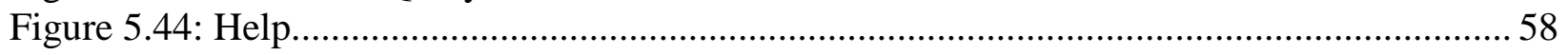

\section{LIST OF TABLES}

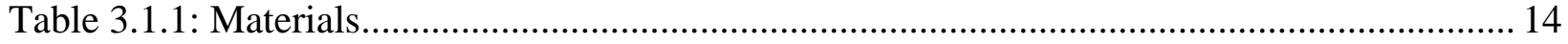

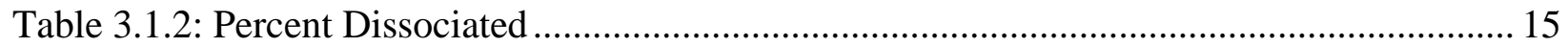

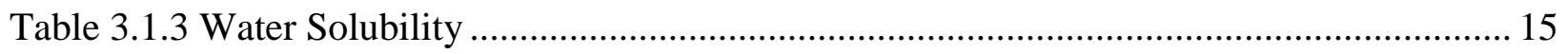

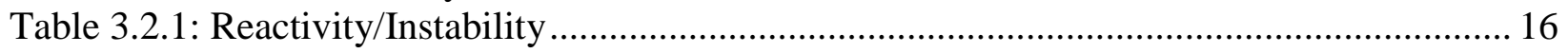

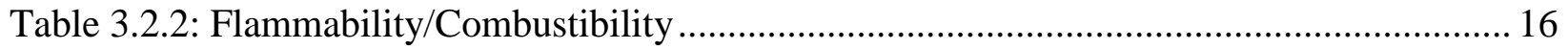

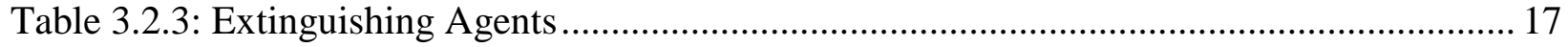

Table 3.2.4: Corrosivity .................................................................................................... 17

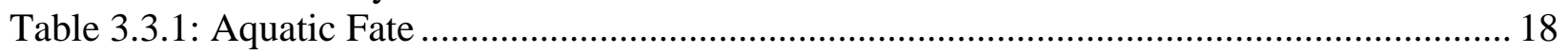

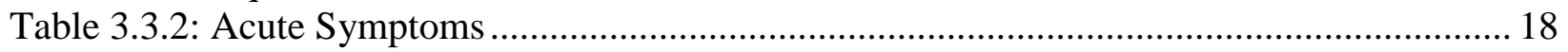

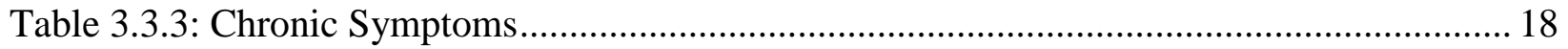

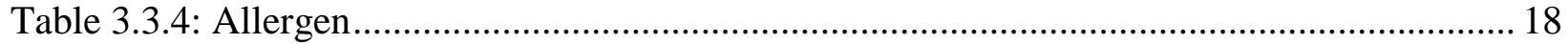

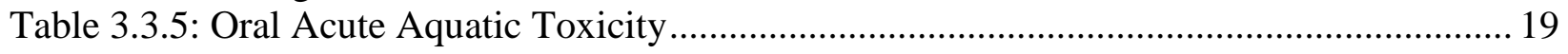

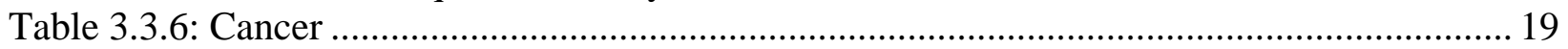

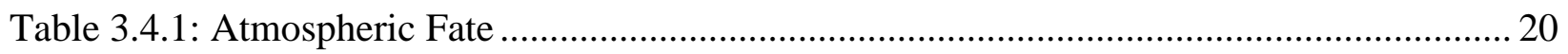

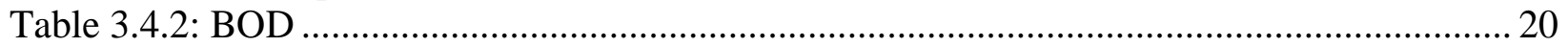




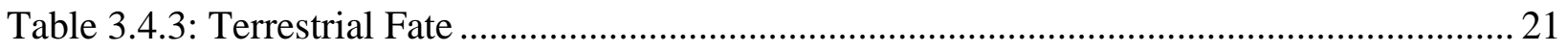

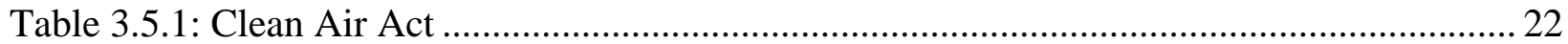

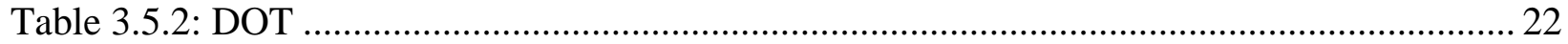

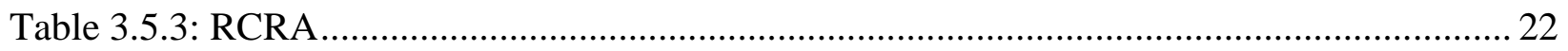

Table 3.6.1: Worker Exposure Criteria (acute-STEL ............................................................ 24

Table 3.6.2: Worker Exposure Criteria (acute-CEIL ............................................................ 24

Table 3.6.3: Worker Exposure Criteria (chronic-TWA) ...................................................... 24

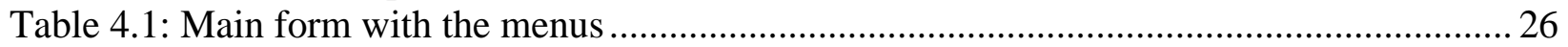

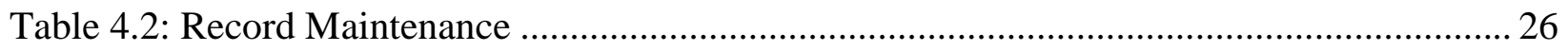

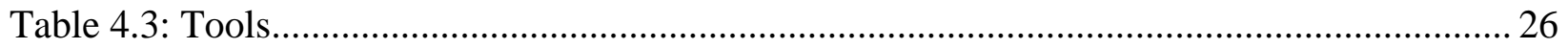




\section{ACKNOWLEDGEMENTS}

I am thankful to my parents for their unyielding support, sacrifices and encouragement throughout my studies. I am greatly indebted to my research advisors, Dr. Rashpal Singh Ahluwalia and Dr. David Whaley for their invaluable guidance throughout this research. The advice and insight provided by Dr. Ahluwalia in all matters pertaining to research have been extremely invaluable. I was fortunate to have him as a teacher in classroom. Dr. Whaley was extremely kind in explaining important aspects of research. His guidance and encouragement have made a difference. I would like to express special thanks to both of them for their generous time and kind co-operation.

I am grateful to Dr. Robert Creese for his valuable comments and suggestions that improved the quality of the report. His suggestions during the initial stages of the research were also valuable. His kindness is very much appreciated.

My heartfelt thanks to Mr. Wiselin Das Mathuram and Mr. Ravi Gottumukkala for helping me solve problems in visual basic during this research. Finally, I thank IMSE department of WVU for their continued financial support, which was of great help in my pursuit for completion of M.S studies. 


\section{CHAPTER 1: INTRODUCTION}

Development of the database structure described in this work was originally motivated by the need for an easily used compendium of the properties of materials encountered in dismantling and recycling or disposal of components of retired marine vessels, here called 'ships'. However, the broader need was recognized to create a database structure which could be useful for many activities, including those where materials encountered could harm people or the environment.

This database structure was developed for use in the United States (US), so it contains referrals to applicable US regulations. However, in principle, similar refernces to the requirement of other jurisdictions could easily be incorporated in the future.

\subsection{DATABASES AND DECISION SUPPORT SYSTEMS}

It is only in recent years that the profound impact of the ongoing explosion of useful information on organizations and individuals has been widely appreciated. Previously, people considered tangible assets whose value could be appraised with reasonable precision [1]. The value of information is harder to define, but nonetheless it can be enormously important. Computers process data to derive and arrange information, and data is often stored in large databases. Complex software called database systems or database management systems have been developed to allow convenient storage and retrieval of data to provide useful information. Until a few years ago, database systems were used almost exclusively in large organizations on mainframes. Today, many people interact with database systems on microcomputers for personal and business applications.

Decision Support Systems (DSS) are computer-based systems that help decision-makers confront ill-structured problems through direct interaction with data and analysis models [3]. In late 1960s and early 1970s, the first DSS began to appear. They were the result of a number of factors such as:

- Emerging computer hardware and software technology

- $\quad$ Research efforts at leading universities 
- A growing awareness of how to support decision making

- A desire for better information

- An increasingly turbulent economic environment and

- Stronger competition pressures.

The principal tenets of a Decision Support System are [2]:

- These systems can be designed specifically to facilitate the decision making processes

- These systems support rather than automate decision making, and

- These systems are able to respond quickly to the changing needs of decision-makers.

Decision Support Systems have to balance three system capabilities, ease of use by non-technical users, access to a wide variety of data, and analysis and modeling in a variety of ways. DSS constitute a significant current frontier in the application of computers. DSS help by expediting access to information that would otherwise not be readily available.

\subsection{NEED FOR AN INTEGRATED DATABASE FOR HAZARDOUS MATERIAL:}

Retirement from use is inevitable for any manufactured or constructed object. Dismantling and disposal are vital parts of Life Cycle Cost (LCC) of a retired object. During dismantling and disposal many things need to be taken into consideration, such as environmental issues and safety regulations. Effects of chemicals released to the environment during dismantling and disposal are a major concern [4], including issues like aquatic fate, terrestrial fate, and atmospheric fate. Within the US, safety regulations set by the Occupational Safety and Health Administration (OSHA), and the Environmental Protection Agency (EPA) have to be strictly followed. A need was recognized for an integrated Hazardous Materials Database (HMD), which has all the above-mentioned characteristics.

\subsection{PROBLEM STATEMENT:}

There is no database which provides broad coverage for the properties of hazardous chemicals, including the applicable safety and environmental regulations, which can assist the user in handling and disposal of a retired object. The HMD is being developed primarily for assisting 
the persons or organizations responsible for handling and disposal of hazardous materials in objects to be dismantled at the end of their useful life. With all of the potential availability among dismantling scenarios, we have recognized the value of assembling a substantial database of recognized properties of materials, hazardous or otherwise, likely to be found on objects to be dismantled and their components recycled or disposed, now and in the future.

During dismantling, and replacing or disposal of components of a retired object the possible effects of chemicals encountered during the process on the people and environment need to be considered. When there is need to determine safe and legal handling and disposal of a particular chemical substance, gathering all its properties (physical, chemical and hazardous), applicable health regulations, and safety and environmental issues is a tedious and a time consuming activity. This led to the idea of developing a database of hazardous chemical substances with all their relevant properties, including health hazards to workers and potential to harm the environment from the chemicals released, and the applicable safety, health and environmental regulations, which would be flexible, easy and rapid for an organization to use, when it is interested in the dismantling and rcycling or disposal of a retired object.

The HMD for chemicals developed here includes metals and nonmetals, as well as organic and inorganic compounds, with access to properties pooled at one place.

\subsection{SOLUTION APPROACH:}

Keeping in mind the above issues the HMD was developed which can hold extensive information about a chemical. The Database was designed by creating tables and linking them with primary keys. There is one main table called the Materials Table, with links to the other tables. The HMD contains a total of 22 tables. Visual Basic (VB) was used as the front-end tool for designing a query engine for the database. VB is flexible, compatible with any database and can be installed on any kind of a computer.

\subsection{OBJECTIVES OF THIS RESEARCH:}

\section{The objectives of this research are to:}

- Design a database for hazardous materials 
- Populate this structure with properties of a sample of chemicals encountered in ship dismantling

- Develop a user interface which facilitates accessing data related to any kind of chemical substance, and

- Apply this database finally to ship dismantling. 


\section{CHAPTER 2: LITERATURE REVIEW}

\subsection{EXISTING CHEMICAL DATA SOURCES}

CHEMICAL INVENTORY DATABASE is a user-friendly tool to help maintain an inventory of chemicals and produce summary documentation [6]. It is designed so that a user can find where a particular chemical is stored. It helps in saving money by not allowing him to purchase a chemical, which is already in use by the company and also in saving time when keeping the inventory up to date and. It makes printing and editing of the listing easy. This database is suitable for small organizations with a moderate level of inventory of several hundred chemicals. It's designed for use, so that everyone authorized in the organization can easily access the inventory information. This database cannot be used by dismantling industry since it is designed only for maintaining inventory of chemicals.

\section{BRETHERICK'S REACTIVE CHEMICAL HAZARDS DATABASE VERSION 2.0 is a} CD ROM, which offers access to information on safe handling and use of reactive chemicals [8]. It includes every chemical for which documented information on reactive hazards has been found. This CD ROM is published by Butterworth-Heinemann, Newton MA.02158 USA. It costs $\$ 554.96$.

SAMPLE CHEMICAL DATABASE is a chemical database consisting of experimental data and correlations of temperature-dependent properties for 1635 pure chemicals [7]. Collected data have been evaluated, correlated, and checked for thermodynamic consistency. From this database we can view data sets, Design Institute for Physical property Data (DIPPR) approved property constants and regressed correlation coefficients for temperature-dependent properties, as well as calculate temperature-dependent properties in any set of units.

\section{CHEMICAL DATABASE DEVELOPED BY UNITED NUCLEAR SCIENTIFIC} EQUIPMENT \& SUPPLIES deals with chemicals that are used in Fireworks, Explosives, Rocket Fuels or explosives in themselves [11]. All of the uses are not given and only the related 
purposes of each are stated. Whenever possible the following information on chemical formula, melting temperature, decomposition temperature, form (liquid, powder, crystal, etc), whether it will explode, whether it is poisonous, and its usage is provided. These chemicals are offered as a guide for information purposes only and cannot be purchased.

\section{LIGAND CHEMICAL DATABASE FOR ENZYMATIC REACTIONS: A LINK BETWEEN ENZYME STRUCTURES AND CHEMICAL REACTIONS: This database is} developed by LIGAND chemical Database for Enzymatic Reactions, which is designed to link enzyme structures with enzyme-catalyzed chemical reactions [10].

GUIDE FOR HANDLING HAZARDOUS MATERIALS: This is designed as a simple guide to Hazardous Materials. United Parcel Service has recognized the need for a manual, which will provide all necessary information, while being simple enough for inexperienced shippers to use [18]. The manual incorporates a large number of products, which do not appear in the official DOT listing. The serial number permits UPS to maintain a record of persons holding copies, with the intent that revision pages will be provided as required.

INDUSTRIAL FIRE HAZARDS HANDBOOK: The purpose of this is to provide a broad yet thorough introduction to major industries and industrial processes, with emphasis on the fire hazards that accompany them. This Handbook, therefore, is intended to complement the NFPA codes and standards, and help the user apply them intelligently. It is meant as a basic reference book, which will serve a broad audience, including fire science students as well as those directly involved in industrial fire protection.

HAZARDOUS MATERIALS HANDBOOK: The design of this handbook enables a firefighter to carry information useful to him primarily during inspections, but also at fires. There are seven headings in the main section of the handbook [19]. These are Name of the material (Description of Flash Point), NFPA 704M ID (The National Fire Prevention Association Classification of the fire hazards of material based on their flash points and boiling points), DOT Classification (The Department of Transportation classification for each hazardous 
substance is noted), Firefighting Procedures (FFP), Explosive Hazards, Water and Air Reactivity, Hazardous Mixtures and Toxicity Hazards

EMERGENCY ACTION GUIDE FOR SELECTED HAZARDOUS MATERIALS: This guide was prepared to help emergency service personnel during the first 30 minutes of an accident involving a spill of a volatile, toxic, gaseous and /or flammable material that is shipped in bulk [20]. General and specific safety procedures to follow are provided in spill guides arranged alphabetically by hazardous material. Each left-hand page identifies a specific hazardous material, outlines its potential hazards and provides immediate action information for fires, spills and first aid. Each right hand page specifies recommended evacuation areas and distances for protecting the public from dangerous concentrations of toxic vapors and explosions. Where applicable, necessary water pollution controls are provided.

HAZARDOUS MATERIALS: This book approaches the study of Hazardous Materials from a frame of reference, which explains why the various materials act and react as they do [21]. This explanation is based on the atomic and molecular structure and the chemical reactivity of the materials. This book communicates through the use of common nomenclature, chemical symbols, and structural formulas. The reader is introduced to the laws and principles governing the behavior of hazardous materials as a background for learning to control the behavior. Frequently encountered materials, which have hazardous properties, are identified, both chemically and practically, rationale for fire fighting is based on both their chemical reactivity and their physical properties. Nationally accepted procedures for identifying hazardous chemicals and methods for crisis -handling of them are summarized.

\section{TOXIC METALS POLLUTION CONTROL AND WORKER PROTECTION: This book} contains those metals and compounds that are most likely to cause poisoning in industry while being processed, and in general environment while the disposal of their process-effluents is being implemented [22]. What is more important and effective in the prevention of toxic metals poisoning is through removal of toxic agents in a manner that positively precludes ingestion or contact by all potential victims, and therein lies the emphasis and main endeavor of the book. In this book are condensed vital data that are scattered and difficult to pull together. Important 
techniques are interpreted and explained by actual case histories. This condensed information will enable the users to establish a sound background for action.

A GUIDE TO WORKING WITH HAZARDOUS MATERIALS - The high degree of concern in the industrial community as well as the public at large, is often needlessly aggravated by unfamiliarity with many of these substances as well as the extent of their presence and potential effects [23]. This book is provided as a resource to help the industrial manager, safety and pollution control engineer establish his/her comprehensive program for information and procedures about hazardous substances in workplace.

\subsection{MAJOR DRAWBACKS OF SOME OF EXISTING DATA SOURCES:}

CHEMICAL STRUCTURES DATABASE: The chemical structures database contains more than 2250 automatically collected chemical structures from the Internet, complete with the information about HTML page addresses. This database is searchable with usual chemical search operations, full structure and sub structure search, formula search name search and so on [5]. This database makes chemical information much more accessible and boosts publication of quality chemical information with attached chemical structures. But it is temporarily disabled.

HAZARDOUS CHEMICAL DATABASE is a resource of approximately 2000 dangerous chemicals [9]. The database contains information about organic chemicals, inorganic chemicals, data, information, laws and regulations, EPA number, name of chemical substances, molecular formula, molecular weight, physical properties, use of chemical, melting point, boiling point, flash point, vapor pressure, water solubility, acute human health effects, safety protection for workers, first aid, spills and emergencies, handling and storage, fire hazards, work place exposure etc. This data can be obtained in the form of a CD ROM. 


\subsection{SOURCES USED TO POPULATE THE HAZARDOUS MATERIALS DATABASE (HMD):}

HAZARDOUS PROPERTIES OF CHEMICAL SUBSTANCES: A wide range of information about hazardous characteristics, toxic properties of chemical substances, target organs and toxicology, cancer causing chemicals, teratogenic substances, flammable and combustile properties of chemical substances, explosive characteristics of chemical substances are obtained [13].

FATE AND EXPOSURE DATA FOR ORGANIC CHEMICALS: For each chemical, the physical properties as well as the environmental fate and monitoring data were identified by conducting searches of the environmental Fate Data Bases of Syracuse Research Corporation (SRC) [14].

CHEMICAL NAMES AND SYNONYMS: This gives a list of CAS Registry Numbers, Simplified Molecular Input Line Entry System (SMILES) notation, chemical names, chemical synonyms and molecular weights of all the chemicals [15].

HAZARDOUS MATERIALS HANDBOOK: Hazardous Materials Handbook covers nearly 1350 hazardous chemicals found in industrial workplace and frequently transported in bulk. It has a record of all the chemical names, their synonyms, physical description, chemical designation, health hazards, fire hazards, chemical reactivity, environmental, shipping information, hazard classification and physical and chemical properties [16].

ENVIRONMENTAL CONTAMINANT REFERENCE DATA BOOK: It is a reference compendium of physical, chemical and biological effects of environmental contaminantsprimarily individual chemicals but also some selected simple and complex mixtures. Each substance is summarized and summaries include information on CAS number, DOT and NIOSH numbers, synonyms various detection limits, environmental transport, fate, and effects (selected narrative information on soil adsorption, volatilization, biodegradation, bioaccumulation, probable exposures and effect types), water hemistry, metabolic pathways, molecular formulas, 
regulatory jurisdictions and authorities, standards, reactions, monitoring methods and international standards [17]. 


\section{CHAPTER 3: DATABASE DESIGN}

There are currently a total of 22 tables in the Hazardous Materials Database (HMD), among which the Materials Table is the main table and all the other tables are sub-tables to it. The user can get a wide range of information regarding the materials, which have been entered such as physical properties, chemical properties, environmental transport and fate properties, toxicity etc. We have initially identified 30 chemicals and categories of chemicals, mostly hazardous, occurring in ship dismantling.

HMD is divided into six major groups:

PHYSICAL PROPERTIES

$>$ CHEMICAL PROPERTIES

$>$ HEALTH AND ECOLOGICAL HAZARDS

$>$ ENVIRONMENTAL CONCERN

$>$ SAFETY AND ENVIRONMENTAL REGULATIONS

$>$ EXPOSURE CRITERIA

\subsection{PHYSICAL PROPERTIES}

At present physical properties are divided into three tables:

1. Materials Table

2. Percent Dissociated Table

3. Water Solubility

\subsubsection{MATERIALS TABLE}

Table 3.1.1 gives the Materials Table and its fields. The various fields are explained below:

CAS \#: It is a unique identifier assigned to each chemical registered with Chemical Abstracts Services (CAS) of the American Chemical Society. This number is used to identify chemicals on the basis of their molecular structure. CAS numbers, in the format $\mathrm{xxx}-\mathrm{xx}-\mathrm{x}$, can be used in 
conjunction with chemical names for positive identification and searching on computerized databases.

Molecular Formula: The formula is in Hill notation, which is given as the number of carbons followed by the number of hydrocarbons followed by any other elements in alphabetical order.

Wiswesser Line Notation: This is a chemical structure representation that can be used for substructure searching, in order to predict a property from that structure.

Smiles Notation: A different chemical structure representation that can be used for substructure searching, in order to predict a property from that structure.

Boiling Point: Boiling Point at 1 atmosphere is the temperature of a liquid when its vapor pressure is 1 atmosphere. It indicates whether a liquid will boil and become gas at any particular temperature and sea-level atmospheric pressure.

Melting Point: The melting/freezing point is the temperature at which a solid changes to a liquid or a liquid changes to a solid.

Molecular Weight: It is the weight of a molecule of the chemical relative to a value of 12 for one atom of carbon.

Henry's constant: The Henry's Law constant $(\mathrm{H})$, is the air/water partition coefficient, which is published in two forms. A non-dimensional $\mathrm{H}$ relates the chemical concentration in the gas phase to its concentration in the water phase. The dimensional $\mathrm{H}$ can be determined by dividing the vapor pressure in atmospheres by the water solubility in mole/m3 to give $\mathrm{H}$ in atm-m3/mole. $\mathrm{H}$ provides an indication of the partition between air and water at equilibrium and also is used to calculate the rate of evaporation from water.

Vapor Pressure: It is defined as the equilibrium pressure of the saturated vapor above the liquid, measured in millimeters of mercury $(760 \mathrm{mmHg}=14.7$ psia) at $20 \mathrm{deg} \mathrm{C}$ unless another 
temperature is specified. The vapor pressure of a chemical provides considerable insight into the transport of a chemical in the environment. The volatility of the pure chemical is dependent upon the vapor pressure, while volatilization from water is dependent upon the vapor pressure and solubility in water.

Log Octanol/Water Partition Coefficient: The octanol water partition coefficient is the ratio of the chemical concentration in Octanol divided by the concentration in water at equilibrium. Occasionally chemical Octanol/water partition coefficients are not calculated because a necessary fragment constant for the chemicals are not available.

Bioconcentration Factor: Certain chemicals due to their hydrophobic nature have a tendency to partition from the water column and bioconcentrate in aquatic organisms. This concentration of chemicals in aquatic organisms is of concern because it can lead to toxic concentrations being reached when the organism is consumed by higher organisms such as wild life and humans. Such bioconcentrations are usually reported as the Bioconcentration factor (BCF), or its $\log$ which is the concentration of chemical in the organism at equilibrium divided by the concentration of the chemical in water.

Oral LD50 rat: LD stands for lethal dose. LD50 is the amount of material given all at once, which causes death of 50\% (one half) of a group of test animals. LD50 is one way to measure short term poisoning potential (acute toxicity) of a material. Here rat is used as the test animal. For example LD50 (oral rat) $5 \mathrm{mg} / \mathrm{kg}$ means that 5 milligrams of that chemical for every onekilogram body weight of the rat, when administered in one dose by mouth causes the death of $50 \%$ of the test group. 
Table 3.1.1- MATERIALS

\begin{tabular}{|l|l|}
\hline Chemical Name & Description \\
\hline CAS \# & Identifier assigned by Chemical Abstaract Society \\
\hline Smiles & Chemical structure representation \\
\hline Classification & Which group the chemical belongs to \\
\hline Wiswesser_Notation & One of chemical structure representation \\
\hline Molecular_Wt & Weight of molecule of chemical \\
\hline Molecular_Formula & Number of carbons and hydrocarbons present \\
\hline Boiling_Point & Temperature at which chemical boils \\
\hline Boiling_Point_Range & Range of temp at which the chemical boils \\
\hline Melting_Point & Temperature at which it melts \\
\hline Melting_Point_Range & Range of temperature at which the chemical melts \\
\hline Log Octanol Water partition coefficient & Conc in octanol/conc in water at equlibrium \\
\hline Density & Density of chemical at 25 deg C \\
\hline VP & Equlibrium pressure of saturated vapor above liquid \\
\hline Henry's constant & It is the air/water partition coefficient \\
\hline Bioconcentration factor & Conc of chemical in organism/conc in water \\
\hline Oral LD50 rat & Dose given causes the death of 50\% of test animals \\
\hline Sources & Sources used to get the information \\
\hline Notes & Notes for the chemical \\
\hline
\end{tabular}

\subsubsection{PERCENT DISSOCIATED}

Table 3.1.2 gives the various fields of Percent Dissociated which are explained below

Dissociation constants: The acid dissociation constant as the negative $\log (\mathrm{pKa})$ is given for chemicals that are likely to dissociate at environmental pHs (between 5 and 9). Chemical classes where dissociation is important include phenols, carboxylic acids, and aliphatic aromatic amines. The degree of dissociation affects such processes as photolysis (absorption spectra of chemicals that dissociate can be considerably affected by $\mathrm{pH}$ ), evaporation from water (ions do not evaporate), soil or sediment adsorption, and bioconcentration. 
Table 3.1.2- PERCENT DISSOCIATED

\begin{tabular}{|l|l|}
\hline Chemical Name & \% Dissociation in aqueous solution \\
\hline $\mathrm{pH} 2$ & \\
\hline $\mathrm{pH} 4$ & \\
\hline $\mathrm{pH} 7$ & \\
\hline $\mathrm{pH} 9$ & \\
\hline $\mathrm{pH} 11$ & \\
\hline
\end{tabular}

\subsubsection{WATER SOLUBILITY}

Table 3.1.3 is Water Solubility, which is explained below

Water Solubility: The water solubility of chemicals provides considerable insight into the fate and transport of a chemical in the environment. High water soluble chemicals, which have a tendency to remain dissolved in the water column and not partition to soil or sediment or bioconcentrate in aquatic organisms, are less likely to volatilize from water and are generally more likely to biodegrade. Low water soluble chemicals are just the opposite; they partition to soil or sediments and bioconcentrate in aquatic organisms, many volatilize more readily from water, and are less likely to be biodegradable. Other fate processes that are or can be affected by water solubility include photolysis, hydrolysis, oxidation, and wash out from the atmosphere by rain or fog. The water solubility Table is shown below.

Table 3.1.3 -WATER SOLUBILITY

\begin{tabular}{|l|l|}
\hline Chemical Name & Description \\
\hline Soluble & If the chemical is soluble (Yes/No) \\
\hline Low & If the solubility of the chemical is low \\
\hline Medium & If the solubility of chemical is medium \\
\hline High & If the solubility of chemical is high \\
\hline
\end{tabular}

\subsection{CHEMICAL PROPERTIES}

Chemical Properties are divided into four tables:

1. Reactivity/Instability

2. Flammability/Combustibility

3. Extinguishing Agents 


\section{Corrosivity}

\subsubsection{REACTIVITY/INSTABILITY}

This Table gives the reactivity of the chemical with oxidizers, titanium, and selenium

Table 3.2.1- REACTIVITY/INSTABILITY

\begin{tabular}{|l|l|}
\hline Chemical Name & Description \\
\hline $\mathrm{Oxy}$ & If the chemical reacts with oxidizers \\
\hline $\mathrm{Ti}$ & If the chemical reacts with titanium \\
\hline $\mathrm{Se}$ & If the chemical reacts with selenium \\
\hline
\end{tabular}

\subsubsection{FLAMMABILITY/COMBUSTIBILITY}

This table gives information about the flash point, auto ignition temperature and the upper and lower exposure limits of the chemical.

Flash Point: It is defined as the lowest temperature at which vapors above a volatile combustile substance will ignite in air when exposed to a flame.

Flammable limits in air: The percent concentration in air (\% by volume) is given for the LEL (lower explosive-flammable limit in air, \% by volume) and UEL (upper explosive-flammable limit in air, \% by volume) at room temperature unless otherwise specified. The values, along with those in Flash Point and Auto ignition temperature give an indication of relative Flammability of the chemical.

Table 3.2.2 -FLAMMABILITY/ COMBUSTIBILITY

\begin{tabular}{|l|l|}
\hline Chemical Name & Description \\
\hline Non Flammable & If chemical is not flammable \\
\hline Flash Point & Lowest temp at which chemical vapor ignites when exposed to flame \\
\hline Auto ignition temp & Lowest temperature at which chemical catches fire \\
\hline LEL percent & Lower exposure limit \\
\hline UEL percent & Upper exposure limit \\
\hline
\end{tabular}




\subsubsection{EXTINGUISHING AGENTS}

Table 3.2.3 gives whether the chemical is used or not used as an extinguishing agent

Table 3.2.3 - EXTINGUISHING AGENTS

\begin{tabular}{|l|l|}
\hline Chemical Name & Description \\
\hline Use & Any extinguishing agent that can be used in the presence of the chemical \\
\hline Not Use & Any extinguishing agent that cannot be used in the presence of the chemical \\
\hline
\end{tabular}

\subsubsection{CORROSIVITY}

This table gives the information about the reactivity of the chemical towards flesh, towards steel and the material, which corrodes that particular chemical.

Table 3.2.4 - CORROSIVITY

\begin{tabular}{|l|l|}
\hline Chemical Name & Description \\
\hline Toward Metal & Corrosivity of the chemical towards metal \\
\hline Toward Flesh & Corrosivity of the chemical towards flesh \\
\hline Towards Glass & Corrosivity of the chemical towards glass \\
\hline
\end{tabular}

\subsection{HEALTH AND ECOLOGICAL HAZARDS}

This group is divided into six tables:

1. Aquatic Fate

2. Acute Symptoms

3. Chronic Symptoms

4. Allergen

5. Oral Acute Aquatic Toxicity

6. Cancer

\subsubsection{AQUATIC FATE}

This section reviews how a chemical will behave if released to fresh, marine, or estuarine surface waters. Field studies or aquatic model ecosystems are used when they provide insight into the overall behavior in water. When field or aquatic ecosystems studies are not available or do not give enough data to make conclusions on the aquatic fate of the chemical, data from appropriate degradation, transport, or monitoring sections will be used to synthesize how a chemical is likely to behave if released to water. 
Table 3.3.1- AQUATIC FATE

\begin{tabular}{|l|l|}
\hline Chemical Name & Description \\
\hline Biodegrade & Breakdown by the action of living things \\
\hline Adsorb to Sediment & Adsorption of chemical to sediment \\
\hline Photolysis & Destruction of materials initiated by reactions by absorption of light \\
\hline Photo -Oxidation & Uses light to initiate reactions that destroy contaminants \\
\hline
\end{tabular}

\subsubsection{ACUTE SYMPTOMS AND TOXICITY}

Acute Symptoms observed when the chemical is taken inside the body either through inhalation or ingestion or adsorption through skin or eye.

Table 3.3.2 - ACUTE SYMPTOMS AND TOXICITY

\begin{tabular}{|l|l|}
\hline Chemical Name & Description \\
\hline Inhalation & Intake into the body through breathing \\
\hline Ingestion & Intake into the body through mouth \\
\hline
\end{tabular}

\subsubsection{CHRONIC SYMPTOMS AND TOXICITY}

These are the chronic symptoms observed when the chemical is inhaled or taken in by ingestion or by adsorption through the skin.

Table 3.3.3 - CHRONIC SYMPTOMS AND TOXICITY

\begin{tabular}{|l|l|}
\hline Chemical Name & Description \\
\hline Inhalation & Intake into the body through breathing \\
\hline Ingestion & Intake into the body through mouth \\
\hline
\end{tabular}

\subsubsection{ALLERGENICITY}

Table 3.3.4 gives the information about whether the chemical is an allergen to skin, respiratory tract or other organs of the body.

Table 3.3.4 - ALLERGENICITY

\begin{tabular}{|l|l|}
\hline Chemical Name & Description \\
\hline Skin & Allergen to skin \\
\hline Respiratory & Allergen to lungs \\
\hline Other & Allergen to other organs \\
\hline
\end{tabular}




\subsubsection{ORAL ACUTE AQUATIC TOXICITY}

Toxicity by ingestion: The $\mathrm{LD}_{50}$ values are those defined by the National Academy of Sciences, committee on Hazardous Materials. $\mathrm{LD}_{50}$ signifies that about $50 \%$ of the animals given the specified dose by mouth will die [14]. The values of Water Fowl $\mathrm{LD}_{50}$ and Fish $\mathrm{LC}_{50}$ for the chemical in table 3.3.5

Table 3.3.5 - ORAL ACUTE AQUATIC TOXICITY

\begin{tabular}{|c|c|}
\hline Chemical Name & Name of the chemical \\
\hline Fish $\mathrm{LC}_{50}$ & Amount when taken will kill the fish \\
\hline Water Fowl LD 50 & When consumed $50 \%$ of the animals will die \\
\hline
\end{tabular}

\subsubsection{CANCER}

A carcinogen is a substance capable of causing cancer. Most cancers are induced by many synthetic or naturally occurring chemicals, which include inorganic substances, organics, hormones, and many solid-state materials.

Mutagens: These cause cell mutations, which may or may not later develop into cancer, birth defects or other health effects.

Genotoxic Carcinogens: Carcinogenic chemicals may alter Deoxyribonucleic acid (DNA) to cause uncontrolled cell replication. DNA is a long macromolecule that carries a genetic code through which genotypic characteristics are inherited. A chemical may interact with DNA through the genetic mechanism, altering the structure or number of the chromosomes, causing gene mutation or duplication. Such substances are known as Genotoxic carcinogens.

Teratogens: These are chemical and physical agents that can cause birth defects and mortality among new born, malformations, growth retardation and functional disorders.

Table 3.3.6 - CANCER

\begin{tabular}{|l|l|}
\hline Chemical Name & Description \\
\hline Mutagen & Causes mutations \\
\hline Genotoxic carcinogen only & Alters genes, can lead to cancer \\
\hline Promoter carcinogen only & Enhances cell proloferation, can lead to cancer \\
\hline
\end{tabular}




\subsection{ENVIRONMENTAL CONCERN:}

This group has three tables under it, which are as follows:

1. Atmospheric Fate

2. BOD

3. Terrestrial Fate

\subsubsection{ATMOSPHERIC FATE}

This section reviews how a chemical will behave if released to atmosphere. The vapor pressure will be used to determine if the chemical is likely to be in the vapor phase or adsorbed to particulate matter. The water solubility will be used to assess the likelihood of washout with rain.

Table 3.4.1 -Atmospheric Fate

\begin{tabular}{|l|l|}
\hline Chemical Name & Description \\
\hline Reactions & Various other chemicals with which it reacts \\
\hline $\mathrm{T}_{1 / 2}$ for $24 \mathrm{hrs}$ & Half life for 24 days \\
\hline $\mathrm{T}_{1 / 2}$ day time & Half life during day \\
\hline $\mathrm{T}_{1 / 2}$ night time & Half life during night \\
\hline $\mathrm{T}_{1 / 2}$ Dry sedimentation & Dry remove half life \\
\hline $\mathrm{T}_{1 / 2}$ Wet deposition & Precipitation washout half life \\
\hline
\end{tabular}

\subsubsection{BOD}

Biological Oxygen Demand (BOD): Also called "biochemical oxygen demand", this is a standard way of describing how much oxygen dissolved in water is consumed by biological oxidation of the chemical during the stated period of time. When given in percent, the values indicate the pounds of chemical during the time stated.

Table 3.4.2 - BOD

\begin{tabular}{|l|l|}
\hline Chemical Name & Description \\
\hline BOD 5 day $\mathrm{mg} / \mathrm{l}$ & Biological oxygen demand for 5day, $\mathrm{mg} / \mathrm{l}$ \\
\hline BOD $_{1 / 2}$ & Biological oxygen demand other formats \\
\hline
\end{tabular}




\subsubsection{TERRESTRIAL FATE}

This section shows how a chemical will behave if released to soil or ground water. Field studies or terrestrial model ecosystem studies are used when they provide insight into overall behavior in soil. Quite often, field or terrestrial ecosystem studies either are not available or do not give enough data to make conclusions on the terrestrial fate of a chemical. In these cases, data from sections on biodegradation, abiotic degradation, soil adsorption/mobility, volatilization from water/soil, and any appropriate monitoring data will be used to synthesize how a chemical is likely to behave if released to soil.

Soil Adsorption/Mobility: For many chemicals experimental soil or sediment partition coefficients are available. These values are measured by determining the concentration in both the solution (water) and solid (soil or sediment) phases after shaking for about 24 to 48 hours and using different initial concentrations.

Table 3.4.3 - TERRESTRIAL FATE

\begin{tabular}{|l|l|}
\hline Chemical Name & Description \\
\hline Aerobic Biodegradation & Biodegradation in the presence of air \\
\hline Anaerobic Biodegradation & Biodegradation in the absence of air \\
\hline Soil adsorption/Mobility & Conc in both solution / solid after shaking for 24 to 48hrs \\
\hline
\end{tabular}

\subsection{SAFETY AND ENVIRONMENTAL REGULATIONS}

This group is divided into three tables:

1. Clean Air Act

2. DOT (U.S Department of Transportation)

3. Resource Conservation and Recovery Act (RCRA, Waste Management)

\subsubsection{CLEAN AIR ACT}

Table 3.5.1 has three fields

HAP stands for Hazardous Air Pollutant

EHS stands for Extremely Hazardous Substance

HRP stands for High Risk Pollutants 
Table 3.5.1- CLEAN AIR ACT

\begin{tabular}{|l|l|}
\hline Chemical Name & Description \\
\hline Criteria Pollutants & Co, $\mathrm{SO}, \mathrm{NO}_{\mathrm{x}}$ etc \\
\hline HAP & Hazardous air pollutants \\
\hline EHS & Extremely hazardous substances \\
\hline HRP & High risk pollutant \\
\hline
\end{tabular}

\subsubsection{DOT}

This is a Hazard identification number that is assigned to the substance by the US Department of Transportation (DOT). This identification number identifies substances regulated by DOT and must appear on shipping documents, the exterior of packages and on specified containers. FIFRA stands for Federal Insecticide Fungicide Rodenticide Act. Table 3.5.2 has following fields

Table 3.5.2 - DOT

\begin{tabular}{|l|l|}
\hline Chemical Name & Description \\
\hline Water pollutant & If it pollutes water \\
\hline Hazard class & Rating given to every chemical \\
\hline Registration pesticide FIFRA & If it can be used as pesticide or insecticide \\
\hline
\end{tabular}

Table 3.5.3 - Resource Conservation and Recovery Act (RCRA, Waste Management)

\begin{tabular}{|l|l|}
\hline Chemical Name & Description \\
\hline Flammability & Flammability of the chemical \\
\hline Reactivity & Reactivity of the chemical \\
\hline Primary valence & Valency of chemical \\
\hline Ion coordination & Ion coordination of chemical \\
\hline Corrosivity & Corrosivity of the chemical \\
\hline Toxicity & Toxicity of the chemical \\
\hline
\end{tabular}

\subsection{EXPOSURE CRITERIA:}

This group is divided into three tables:

1. Worker Exposure Criteria (acute- STEL), Short-Term Exposure Limit

2. Worker Exposure Criteria (acute-CEIL), Ceiling Exposure Limit

3. Worker Exposure Criteria (chronic-TWA), Time-Weighted Average Exposure Limit 


\section{Exposure Limits:}

These are legally enforceable airborne permissible exposure limits (PELs) from OSHA, and recommended airborne exposure limit guidelines called recommended exposure limits (RELs) from National Institute of Ocupational Safety and Health (NIOSH) or called threshold limit values (TLVs) from the American Conference of Government Industrial Hygenists (ACGIH) or called Work Place Environmental Exposure Levels (WEEL) from the American Industrial Hygenists Association.

There are 3 kinds of TLVs. A time weighted average TLV (TLV-TWA) is a concentration in breathing air for a normal 8-hour workday and a 40-hour workweek, to which it is believed that nearly all workers may be exposed, day after day without adverse effects. A ceiling TLV value (TLV-C) is the concentration that should not be exceeded during any part of the working exposure. If instantaneous monitoring is not feasible, then the (TLV-C) can be assessed by sampling over 15-minute periods except for those substances that may cause immediate irritation when exposures are short. A short-term exposure limit (TLV-STEL) is a 15-minute TWA exposure concentration in air, which should not be exceeded at any time in a workday, even if the 8-hour TWA is within the TLV-TWA. Exposures above the 8 hour TLV-TWA up to the TLV-STEL should not last more than 15 minutes and should not occur more than 4 times in a work day. This should be atleast 60 minutes between successive exposures in the range.

There are 3 kinds of PELs. A time weighted average PEL (PEL-TWA) is a concentration that must not be exceeded during any 8-hour shift or 40-hour workweek. An OSHA ceiling concentration must not be exceeded during any part of the workday; if instantaneous monitoring is not feasible, the ceiling must be assessed as a 15 minute TWA exposure. A short-term exposure limit PEL (PEL_STEL) is defined similarly to a TLV STEL.

All WEELS are expressed as either time weighted average (TWA) concentrations or ceiling values, however, different time periods are specified depending on the properties of the agent. An 8-hour TWA indicates a time weighted average concentration for a normal 8-hour workday and a 40-hour workweek. A ceiling limit should not be exceeded at any time during the 
workday. A short-term TWA is a time weighted average concentration of shorter duration (such as 15 minutes) established to limit excursion levels.

All the above-discussed values for a chemical are tabulated which can be seen from tables 3.6.1, 3.6.2 and 3.6.3.

Table 3.6.1 - WORKER EXPOSURE CRITERIA (ACUTE-STEL) mg/m

\begin{tabular}{|l|l|}
\hline Chemical Name & Description \\
\hline TLV & Threshold limit value \\
\hline WEEL & Workplace environmental exposure level \\
\hline
\end{tabular}

Table 3.6.2 - WORKER EXPOSURE CRITERIA (ACUTE-CEIL) mg/m

\begin{tabular}{|l|l|}
\hline Chemical Name & Description \\
\hline PEL & Permissible exposure limit \\
\hline TLV & Threshold limit value \\
\hline WEEL & Workplace environmental exposure level \\
\hline
\end{tabular}

Table 3.6.3 - WORKER EXPOSURE CRITERIA (CHRONIC-TWA) mg/m

\begin{tabular}{|l|l|}
\hline Chemical Name & Description \\
\hline TLV & Threshold limit value \\
\hline WEEL & Workplace environmental exposure level \\
\hline PEL (respirable) & Permissible exposure limit \\
\hline
\end{tabular}




\section{CHAPTER 4: IMPLEMENTATION}

Microsoft Access is a powerful, yet intuitively easy-to-use, database management system, designed to run in the Windows environment. Its primary purpose is to provide an efficient system for storing large amounts of data - a system in which any one piece of information can be quickly located. Additionally, relationships between data can be established allowing one to additionally retrieve the related information of any one piece of information requested. Access includes many features for manipulating the information that is stored. Using the program, one can

- Sort and rearrange information in various ways

- Extract and work with subsets of the information.

The Database contains a main table and several sub tables under it. These are linked with the help of Primary keys and foreign keys. In HMD the chemical name was made the primary key since most chemicals have a unique name. The relationships diagram can be seen from appendix 26 at the end.

\subsection{THE QUERY ENGINE:}

A Query engine was developed to assist the user in creating and retrieving data for HMD. Visual Basic was selected to be the front end for accessing data from HMD. It is flexible and compatible with any kind of database. The Graphical User Interface (GUI) an inherent feature of Visual Basic, uses illustrations for text, which enable users to interact with the application.

TABLES SHOWING THE MAIN MENUS THEIR CORRESPONDING FIELDS AND SUB FIELDS: 
Table 4.1: MAIN FORM WITH MENUS

\begin{tabular}{|l|l|l|}
\hline File & Tools & Help \\
\hline Record Maintenance & Search & Help file \\
\hline Exit & Query & \\
\hline
\end{tabular}

Table 4.2: RECORD MAINTENANCE

\begin{tabular}{|c|c|c|c|c|c|}
\hline $\begin{array}{l}\text { Physical } \\
\text { Properties }\end{array}$ & $\begin{array}{l}\text { Chemical } \\
\text { Properties }\end{array}$ & $\begin{array}{l}\text { Health } \\
\text { Hazards }\end{array}$ & $\begin{array}{l}\text { Safety } \\
\text { Regulations }\end{array}$ & $\begin{array}{l}\text { Environmental } \\
\text { Concern }\end{array}$ & $\begin{array}{l}\text { Exposure } \\
\text { Criteria }\end{array}$ \\
\hline Materials & $\begin{array}{l}\text { Reactivity } \\
\text { /Instability }\end{array}$ & Aquatic Fate & $\begin{array}{l}\text { Clean Air } \\
\text { Act }\end{array}$ & $\begin{array}{l}\text { Atmospheric } \\
\text { Fate }\end{array}$ & $\begin{array}{l}\text { Worker } \\
\text { exposure } \\
\text { criteria } \\
\text { (STEL) }\end{array}$ \\
\hline $\begin{array}{l}\text { Percent } \\
\text { Dissociated }\end{array}$ & $\begin{array}{l}\text { Flammability } \\
\text { /combustibility }\end{array}$ & $\begin{array}{l}\text { Acute } \\
\text { symptoms }\end{array}$ & DOT & $\begin{array}{l}\text { Biological } \\
\text { Oxygen } \\
\text { demand (BOD) }\end{array}$ & $\begin{array}{l}\text { Worker } \\
\text { exposure } \\
\text { criteria } \\
(\text { CEIL) }\end{array}$ \\
\hline \multirow[t]{4}{*}{$\begin{array}{l}\text { Water } \\
\text { Solubility }\end{array}$} & $\begin{array}{l}\text { Extinguishing } \\
\text { Agents }\end{array}$ & $\begin{array}{l}\text { Chronic } \\
\text { symptoms }\end{array}$ & $\begin{array}{l}\text { Characteristic } \\
\text { RCRA }\end{array}$ & Terrestrial Fate & $\begin{array}{l}\text { Worker } \\
\text { exposure } \\
\text { criteria } \\
\text { (TWA) }\end{array}$ \\
\hline & Corrosivity & Allergen & & & \\
\hline & & $\begin{array}{l}\text { Oral Acute } \\
\text { Aquatic Tox }\end{array}$ & & & \\
\hline & & Cancer & & & \\
\hline
\end{tabular}

Table 4.3: TOOLS

\begin{tabular}{|l|l|}
\hline \multicolumn{1}{|c|}{ Search } & \multicolumn{1}{c|}{ Query } \\
\hline By CAS \# & On Molecular weight \\
\hline By Chemical Name & On Boiling Point \\
\hline By Molecular Formula & On Melting Point \\
\hline & On Vapor Pressure (VP) \\
\hline & On classification \\
\hline & Print \\
\hline
\end{tabular}

Help: helps the user in using the database

Exit: exits the program

The first screen of HMD Query Engine is shown in Figure 4.1. There are two buttons 1."ENTER" button, which is to enter into the database, and the "EXIT" Button, which is used to end the program. 
On clicking the "ENTER" button a new form is opened as shown in Figure 4.2

It asks the user to enter a password. This feature is included for security reasons; only authorized users can access the database

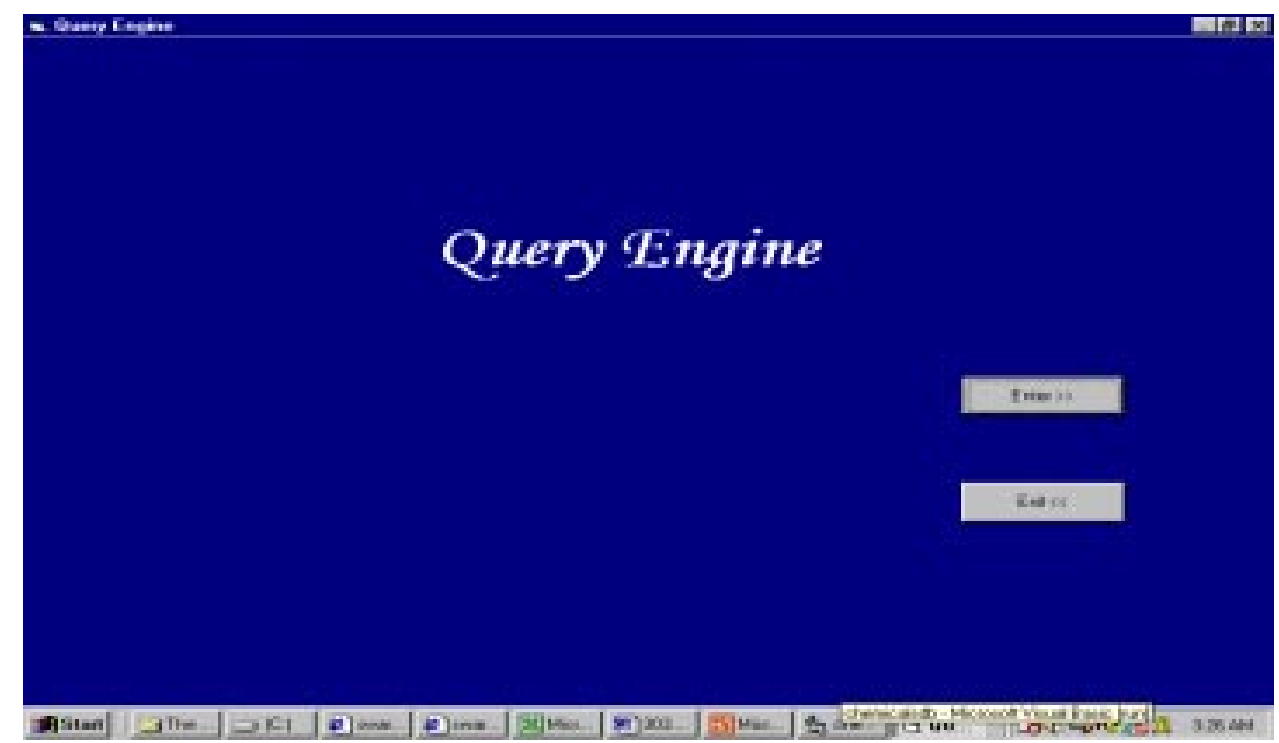

Figure 4.1: HMD Query Engine

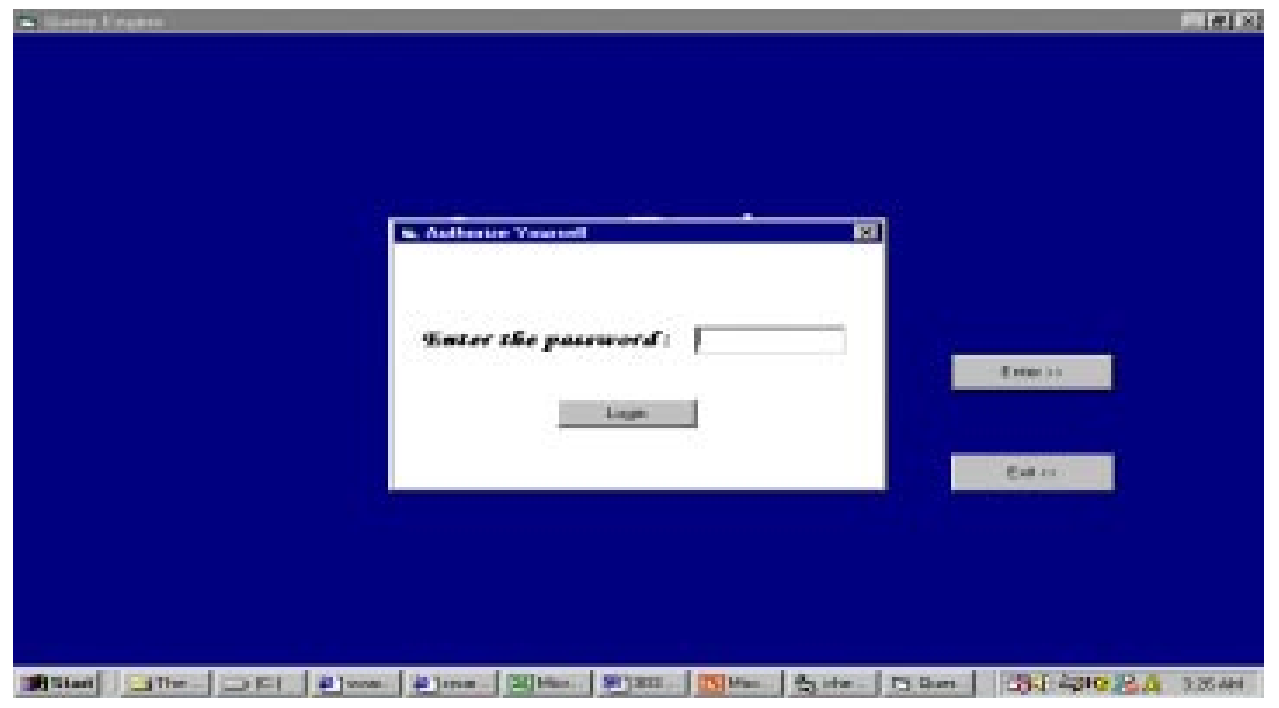

Figure 4.2: Password Form

There is a "LOGIN" button, which should be clicked after typing in the password. If an Invalid Password is entered then a message saying "Invalid Password" appears on the screen as shown in 
Figure 4.3. If correct password is typed, then the form named home is displayed. The menu items of the home form are

- File

- Tools

- Help

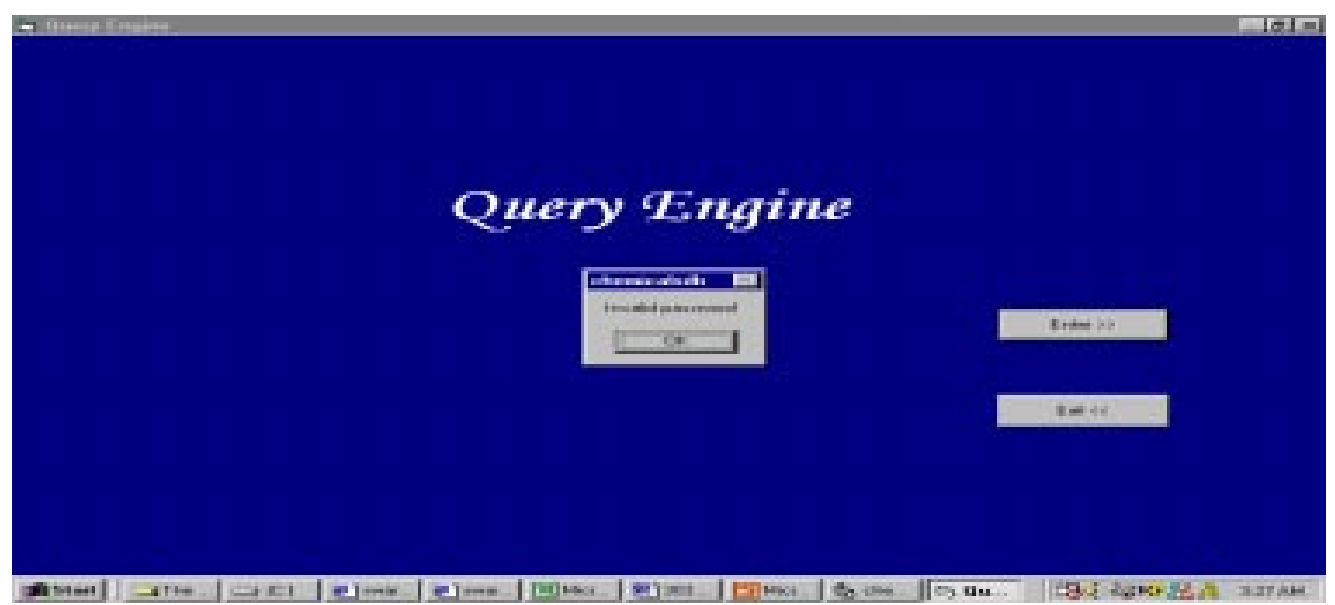

Figure 4.3: Screen showing Invalid Password

When the mouse is placed on top of File, it displays the following pull down menu Figure 4.4

1. Record Maintenance

2. Exit

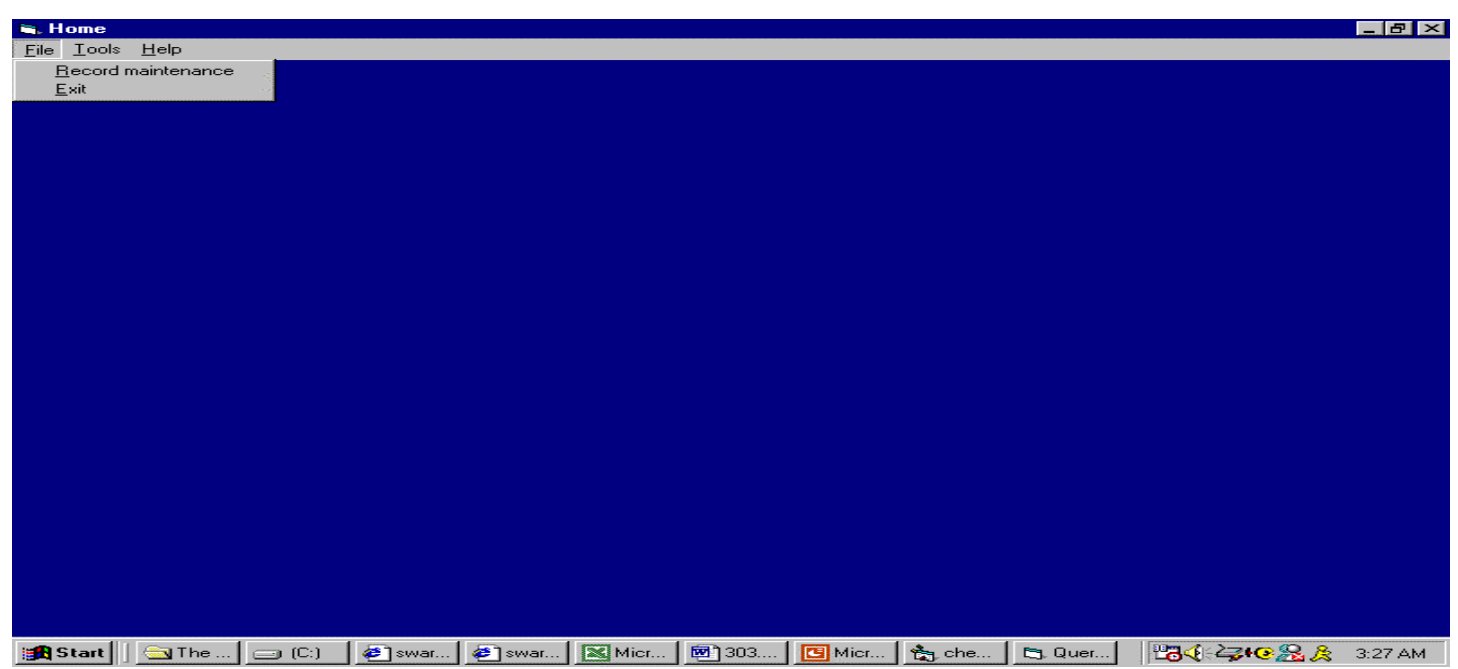

Figure 4.4: Home form with the pull down menu for the file displayed 
On the home form when user clicks on the Record Maintenance of the file pull down menu option, another pull down menu, showing the following properties appears Figure 4.5.
a) Physical properties
b) Chemical Properties
c) Health and Ecological Hazards
d) Exposure Criteria
e) Safety and Environmental Regulations and
f) Environmental concern

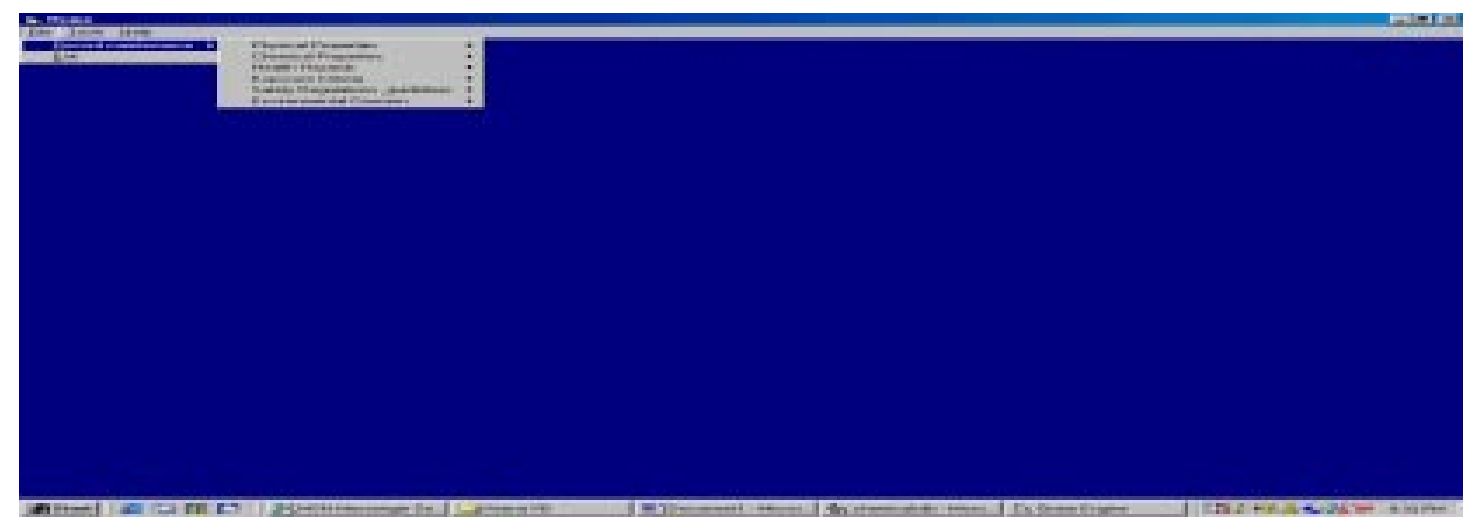

Figure 4.5: Showing the pull down menu of Record Maintenance

Clicking on the physical properties leads to another pull down menu, which has the following:

1) Materials

2) Water solubility and

3) Percent Dissociated tables as shown in the Figure 4.6 below

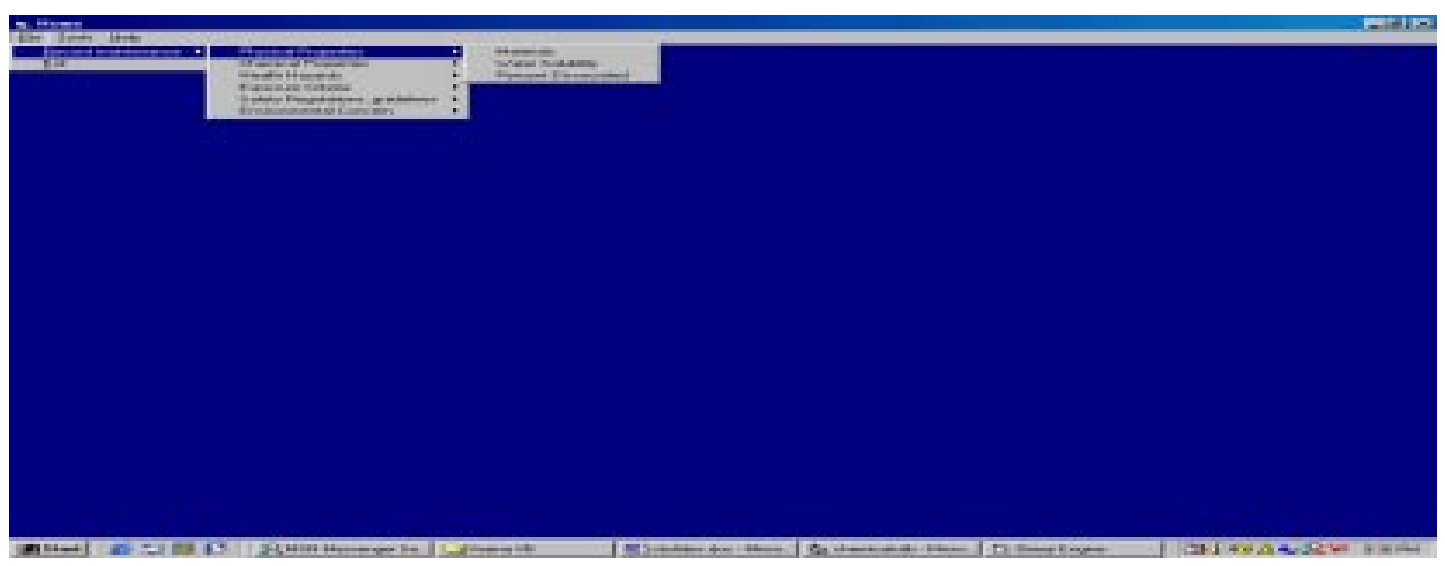

Figure 4.6: Listing the items in pull down menu for Physical Properties 
Clicking on the chemical properties leads to another pull down menu of the following items:

1) Flammability/Combustibility

2) Extinguishing Agents

3) Reactivity/ Instability and

4) Corrosivity

as shown in the Figure 4.7 below

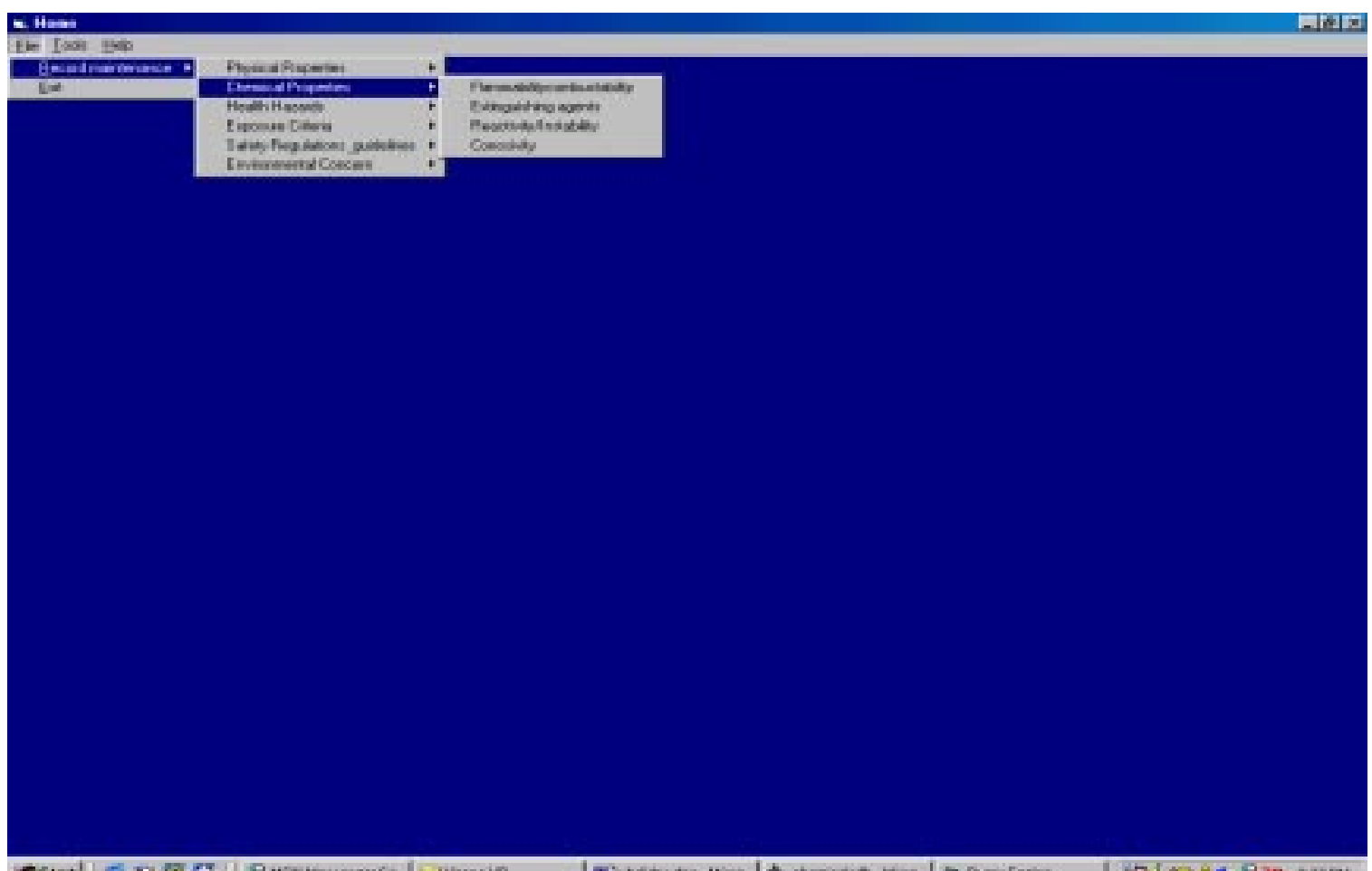

Figure 4.7: Showing the list of items in the pull down menu for Chemical Properties

Clicking on the Health Hazards leads to another pull down menu of the following items:

1) Cancer

2) Aquatic Fate

3) Acute Symptoms and Toxicity

4) Chronic Symptoms and Toxicity

5) Allergenicity and

6) Oral acute aquatic Toxicity

as shown in the Figure 4.8 below 


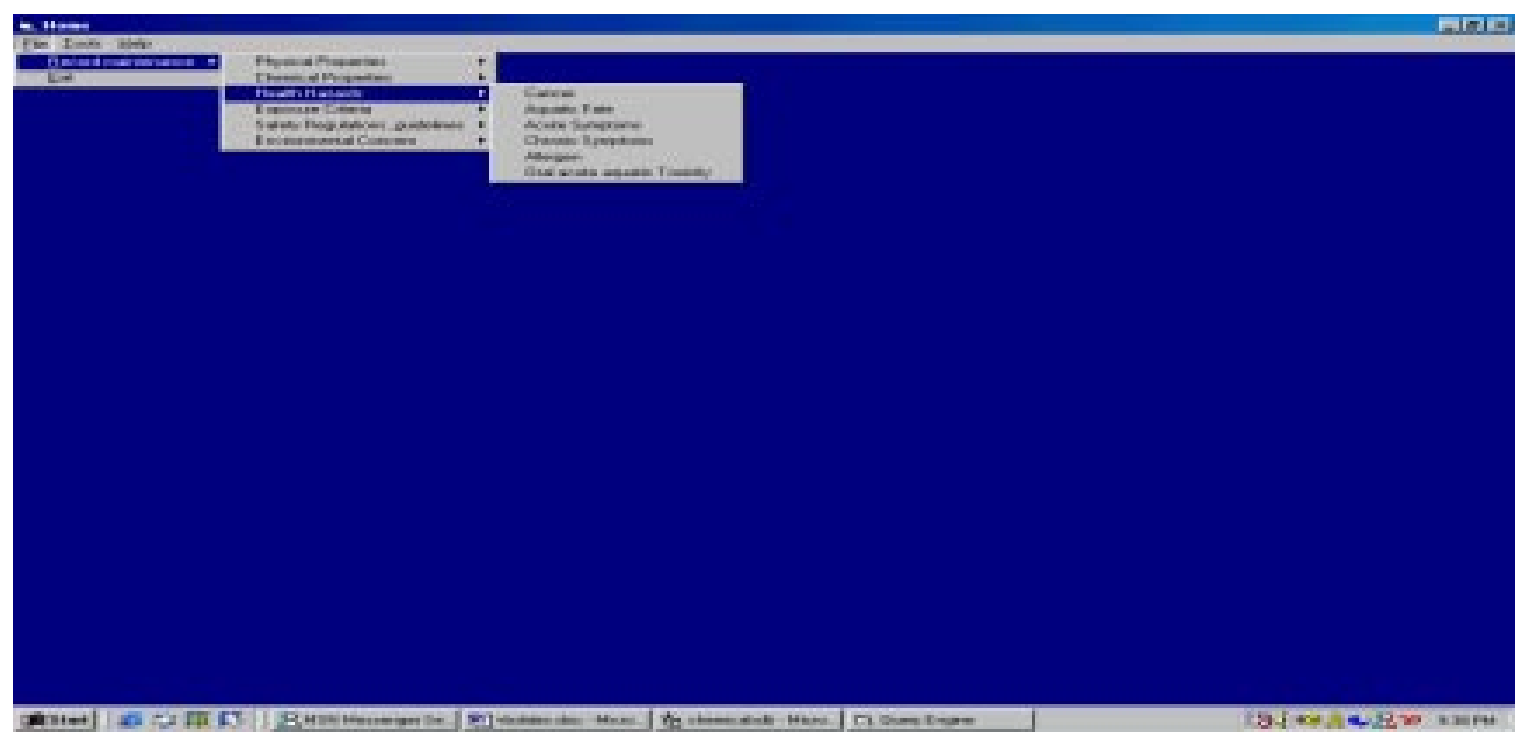

Figure 4.8: showing the pull down menu for Health Hazards

Clicking on the Safety Regulations and guidelines on the Record Maintenance leads to another pull down menu of the following items:

1) $\mathrm{DOT}$

2) Clean Air Act and

3) RCRA

which are shown in the Figure 4.9 below

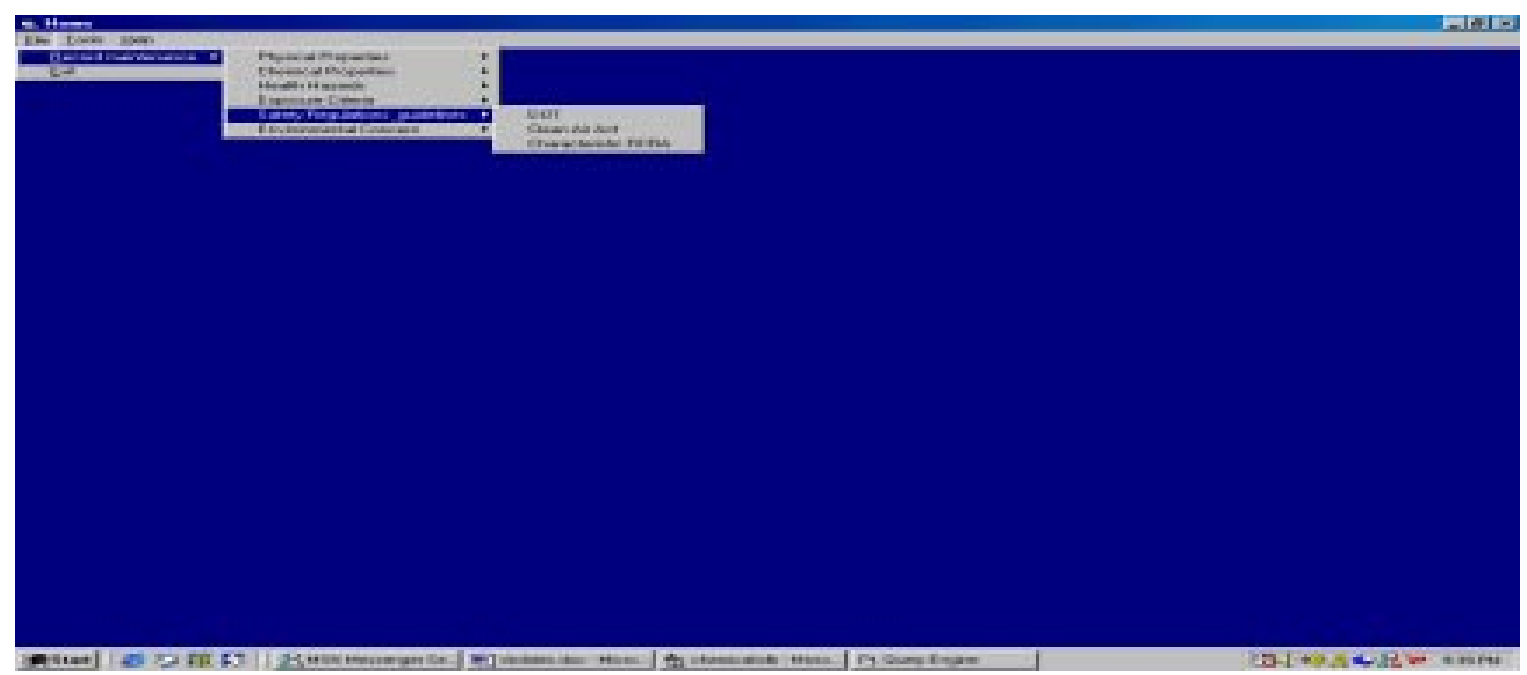

Figure 4.9: Showing the pull down menu for Safety and Environmental Regulations 
Clicking on the Exposure Criteria leads to another pull down menu of the following items:

1) Worker Exposure Criteria (acute-STEL)

2) Worker Exposure Criteria (acute-CEIL) and

3) Worker Exposure Criteria (chronic- TWA)

as shown in the Figure 4.10 below

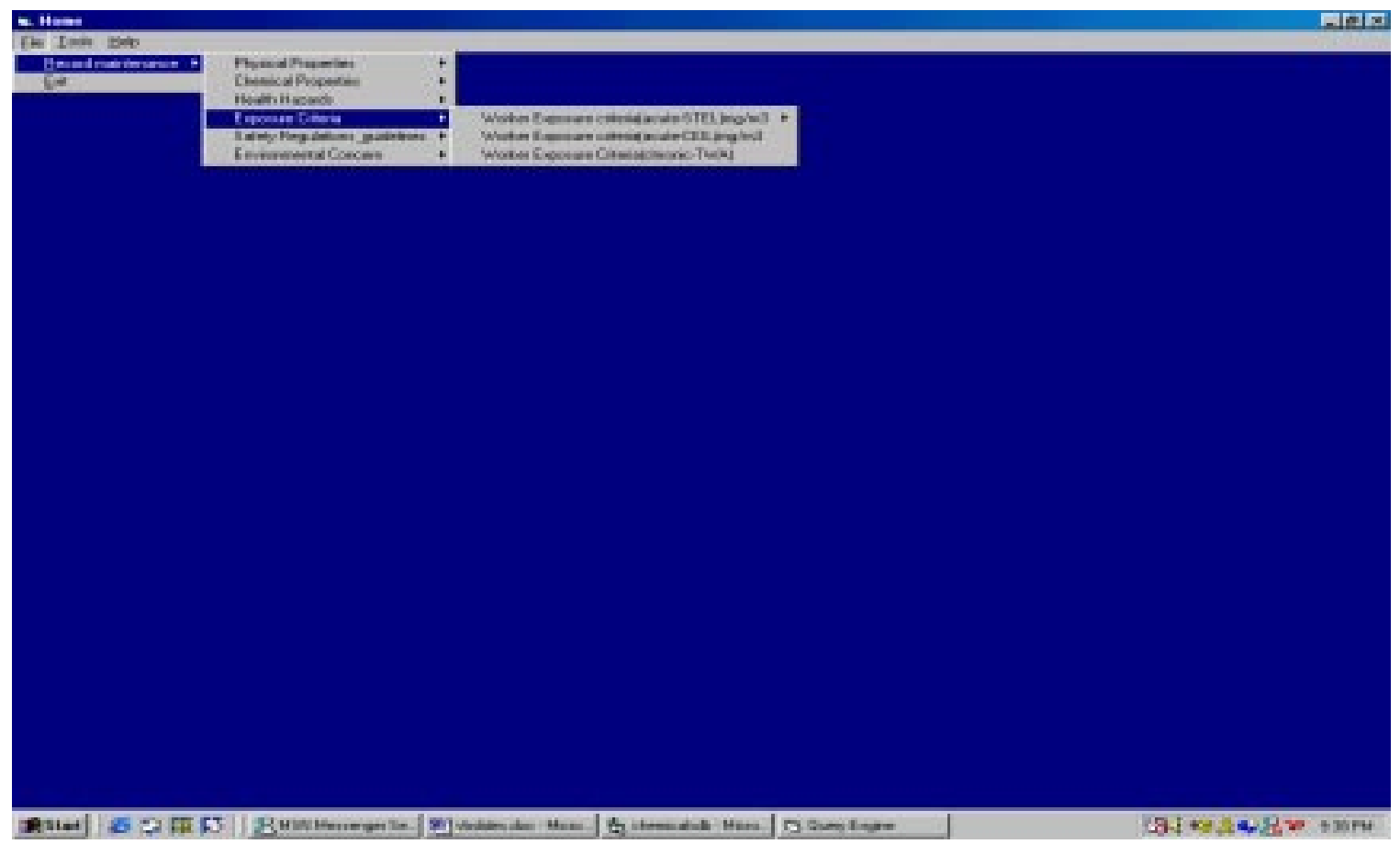

Figure 4.10: Showing the pull down menu for Exposure Criteria

Clicking on the Environmental Concern on the Record Maintenance menu selection leads to another pull down menu of the following:

1) Toxicity

2) Atmospheric Fate

3) Terrestrial Fate and

4) $\mathrm{BOD}$

which is shown in the Figure 4.11 below 


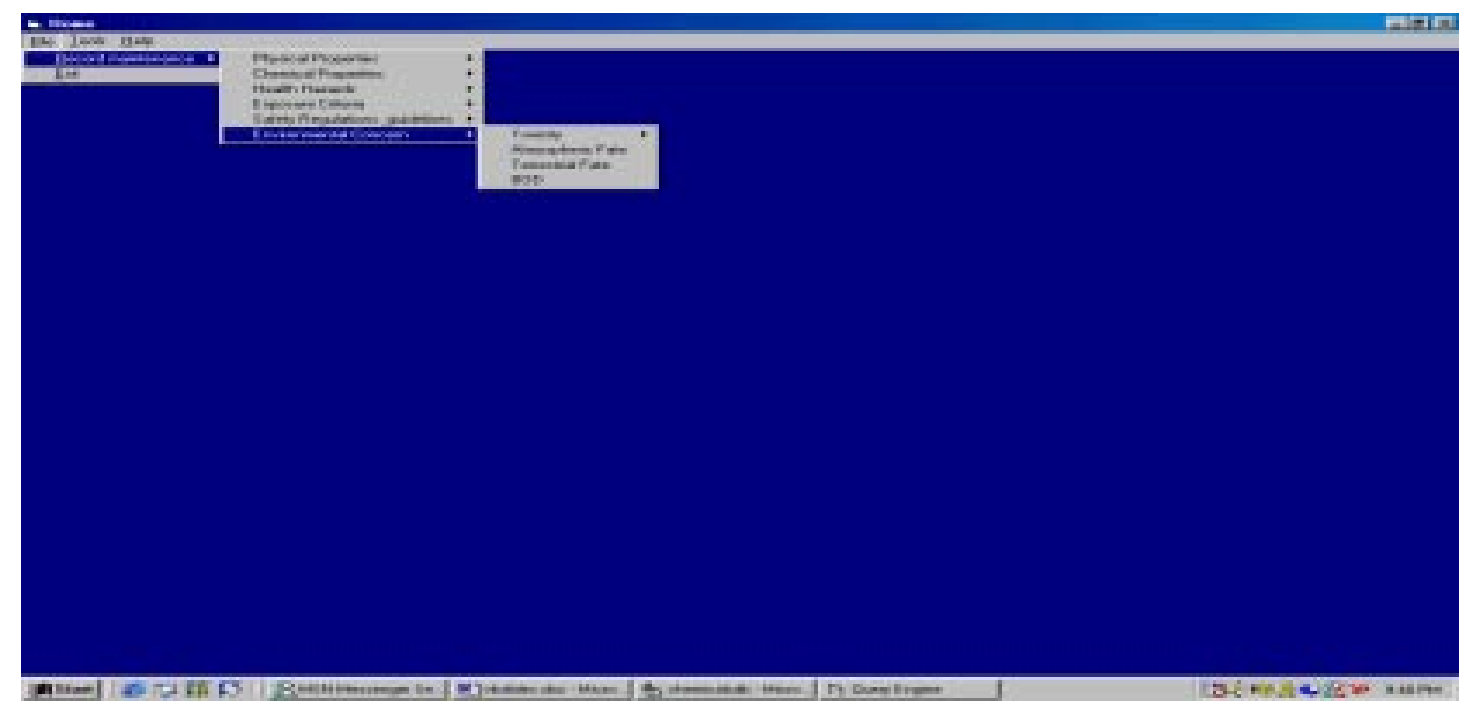

Figure 4.11: Showing the pull down menu for Environmental Concern

When the user clicks on the menu item Search, of the Tools, a form is displayed with the following three options
a) By CAS\#
b) By Chemical Name
c) By Molecular Formula as shown in Figure 4.12 below

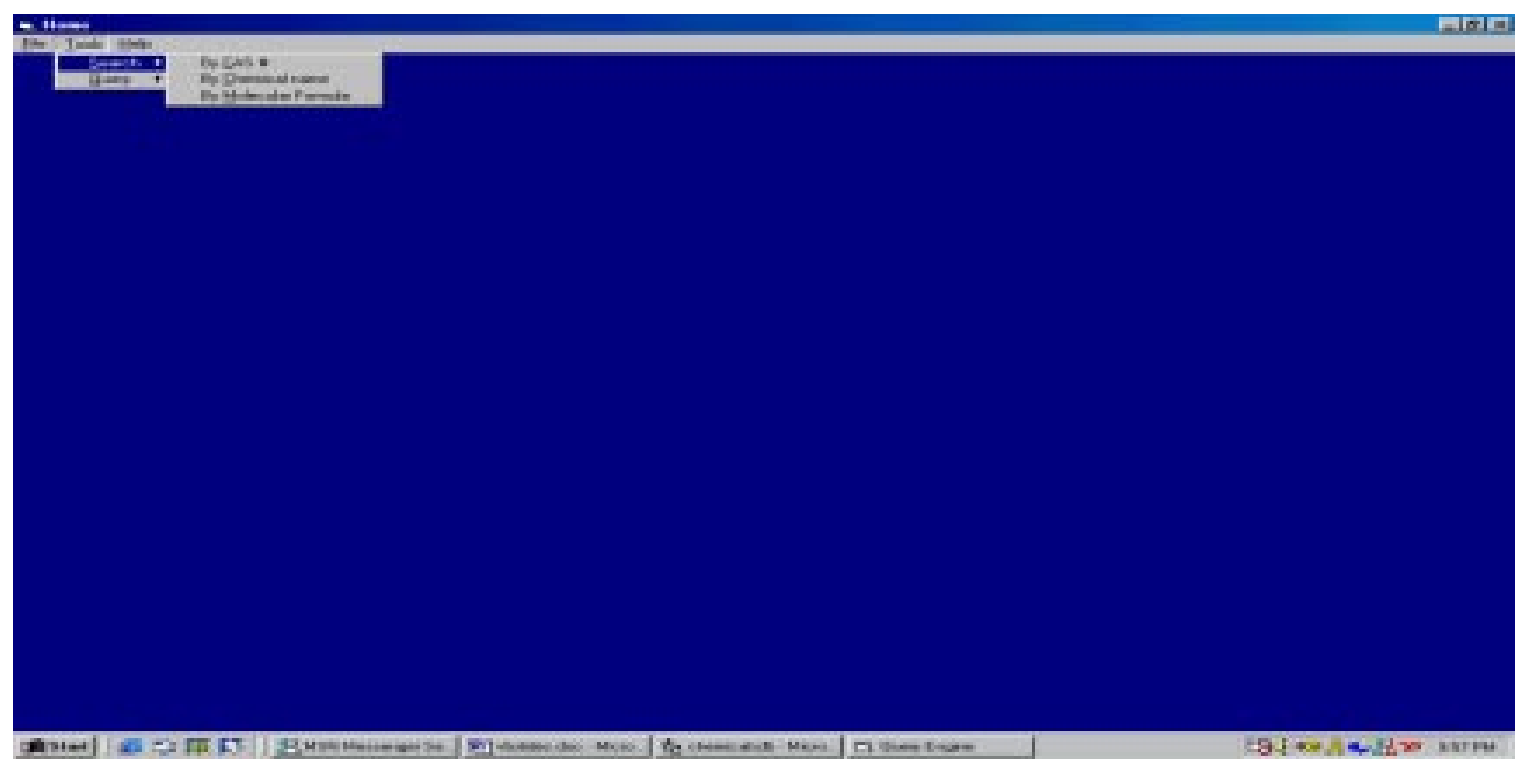

Figure 4.12: Showing the pull down menu for Search 
When the user clicks on the menu item Query of the Tools, the form as shown in Figure 4.13 below is displayed with the following seven options

d) On Molecular Weight

e) On Boiling point

f) On Melting point

g) Molecular formula

h) Save query as and

i) Print

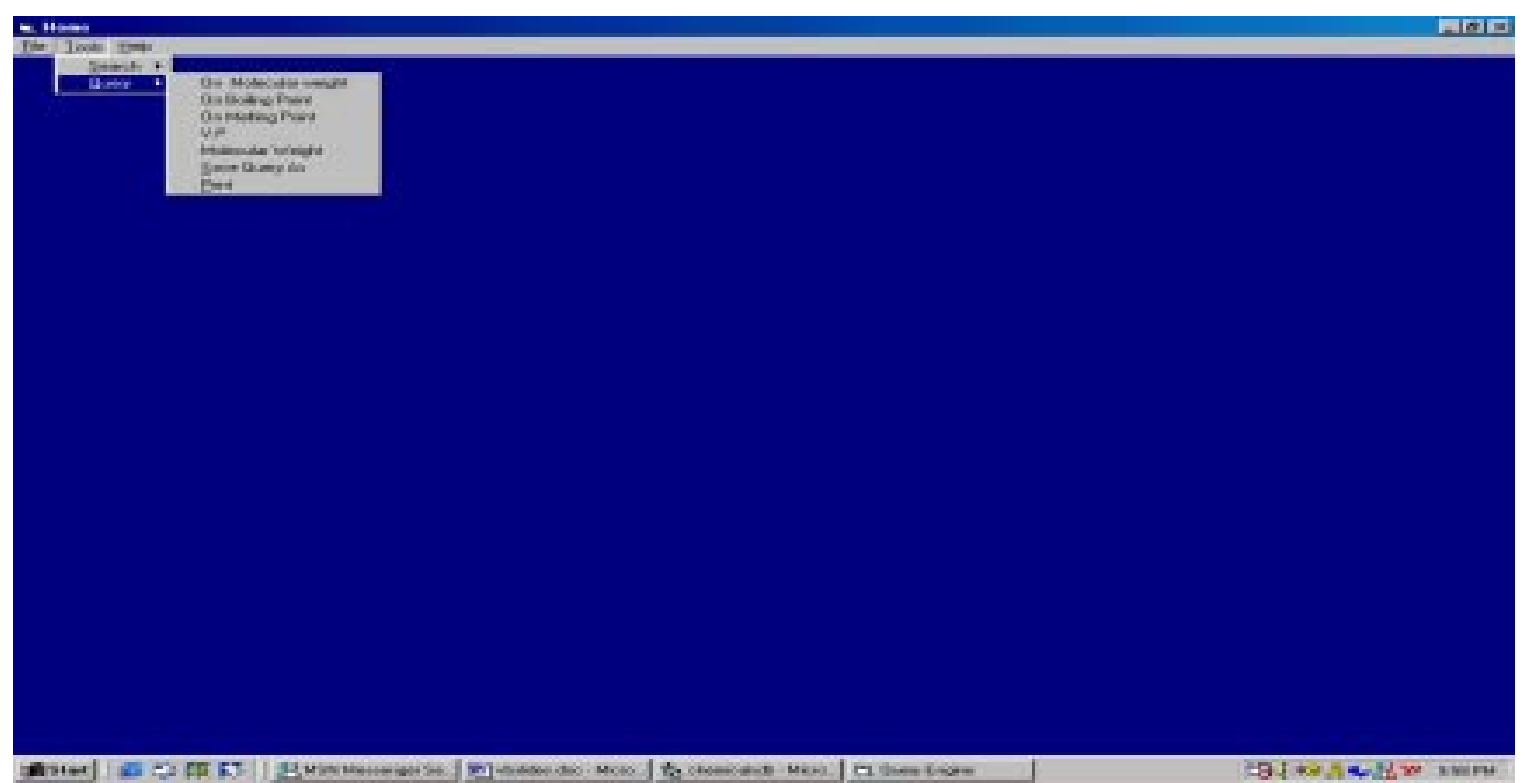

Figure 4.13: Showing the pull down menu for query

HELP: The Help option in the main menu helps the user in using the frontend for the Hazardous Materials Database effectively. It guides the user by providing information about all the forms and how to use them. 


\section{CHAPTER 5: APPLICATION}

\subsection{SHIP DISPOSAL APPLICATION:}

The database can be applied to handling and disposal of many kinds of retired objects. The object may be as simple thing as a pen or as a complex as a ship. During dismantling of a ship many hazardous substances are released. These are of major concern to workers, communities and the environment, so they should be dealt with carefully during disposal. Chemical substances like asbestos, cadmium, lead, and tributyl tin are released. To illustrate how we can utilize the Hazardous Material Database (HMD) we will use as an example a small sample of the above-mentioned chemicals, and apply the HMD to it.

\section{Physical Properties:}

We can get the Physical properties of the chemical from the Materials Table, Percent Dissociated table and the Water Solubility Table. From the HMD database Molecular Weight of Cadmium is found to be 112.4. Its Melting Point $321 \mathrm{deg} \mathrm{C}$ and Boiling Point is $767 \mathrm{deg} \mathrm{C}$. Vapor pressure is $0.12 \mathrm{mg} / \mathrm{m} 3$ at $25 \mathrm{deg} \mathrm{C}$.

When the user clicks on the menu item Materials of the Physical Properties pull down menu the form shown in Figure 5.1 is displayed. All the tables in the database can be accessed through the front end. The user can add, update, delete and refresh the records in the database.

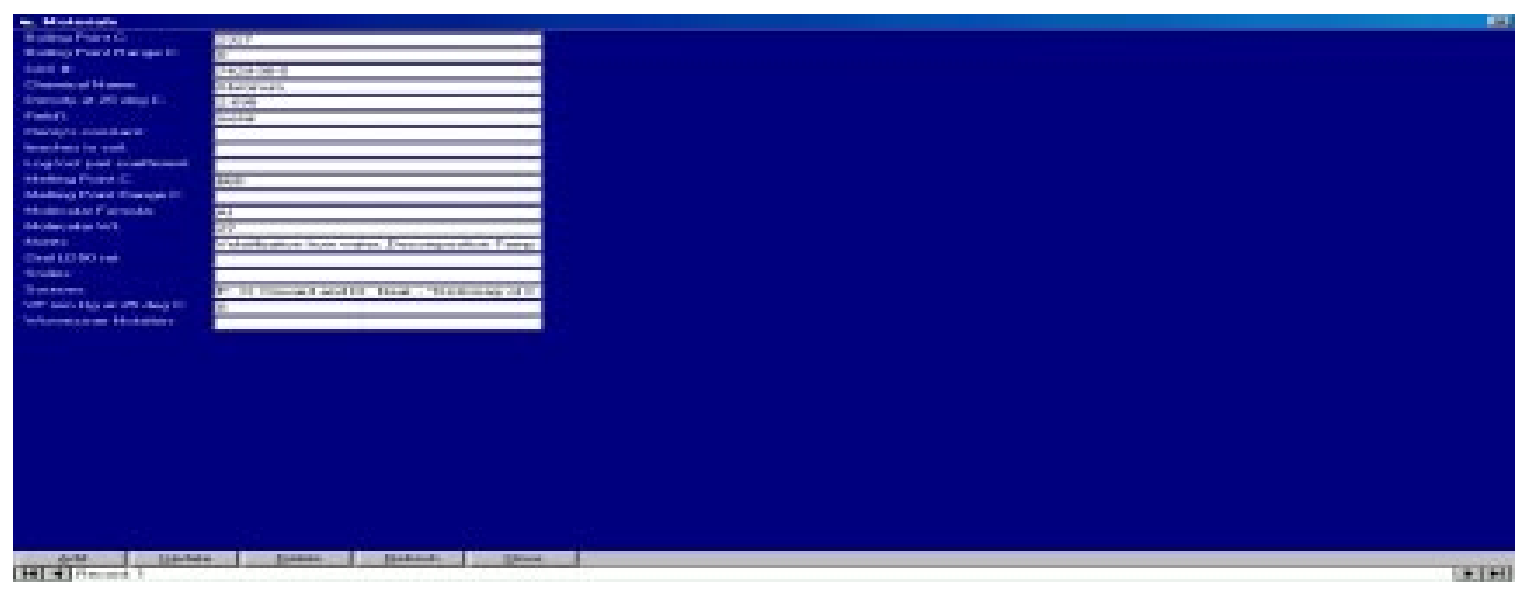

Figure 5.1: Physical Properties 
When the user clicks on the menu item Water Solubility of the Physical Properties, the form shown in Figure 5.2 is displayed

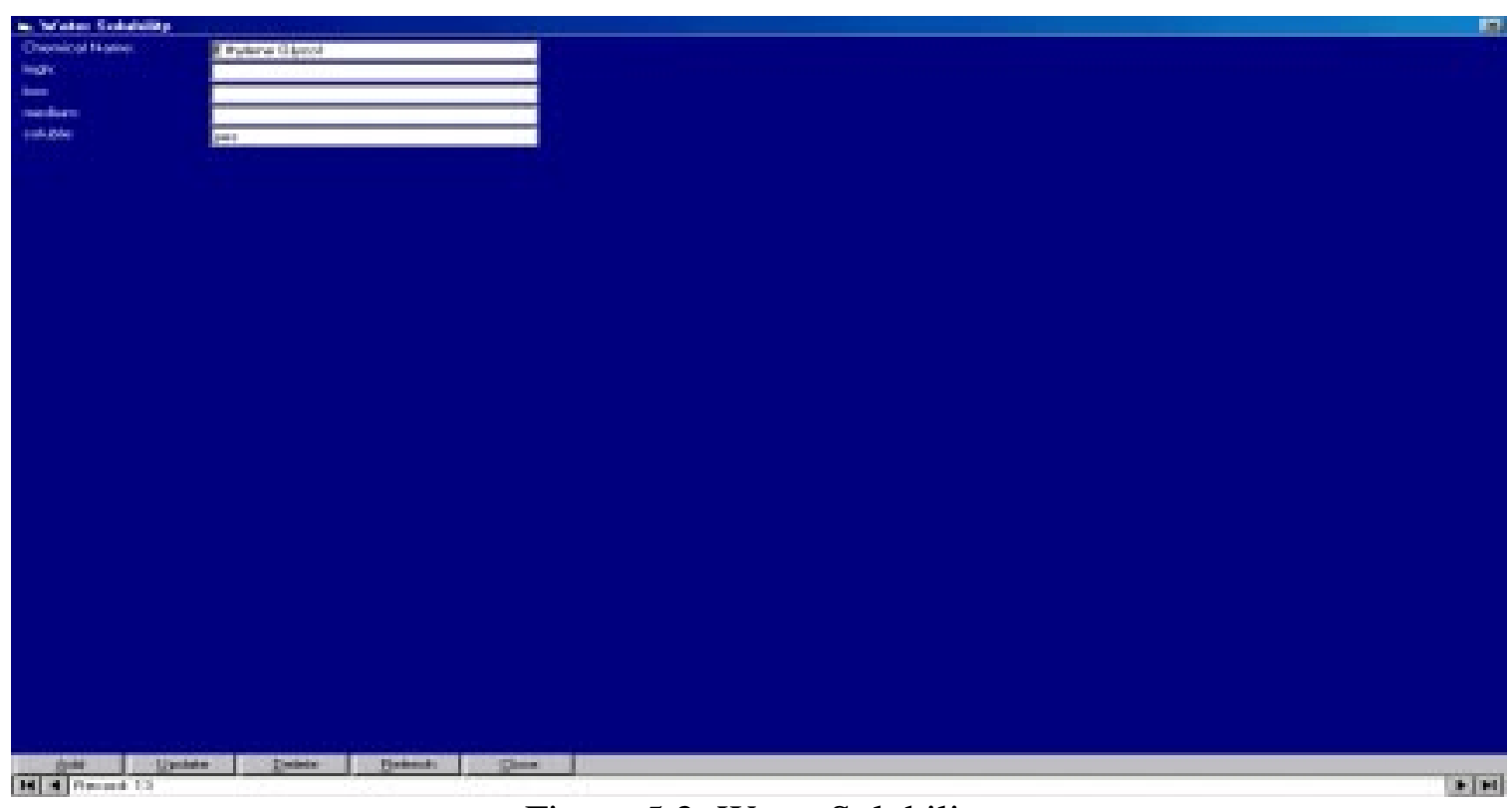

Figure 5.2: Water Solubility

When the user clicks on the menu item Percent Dissociated of the Physical Properties, the form as shown in Figure 5.3 below is displayed

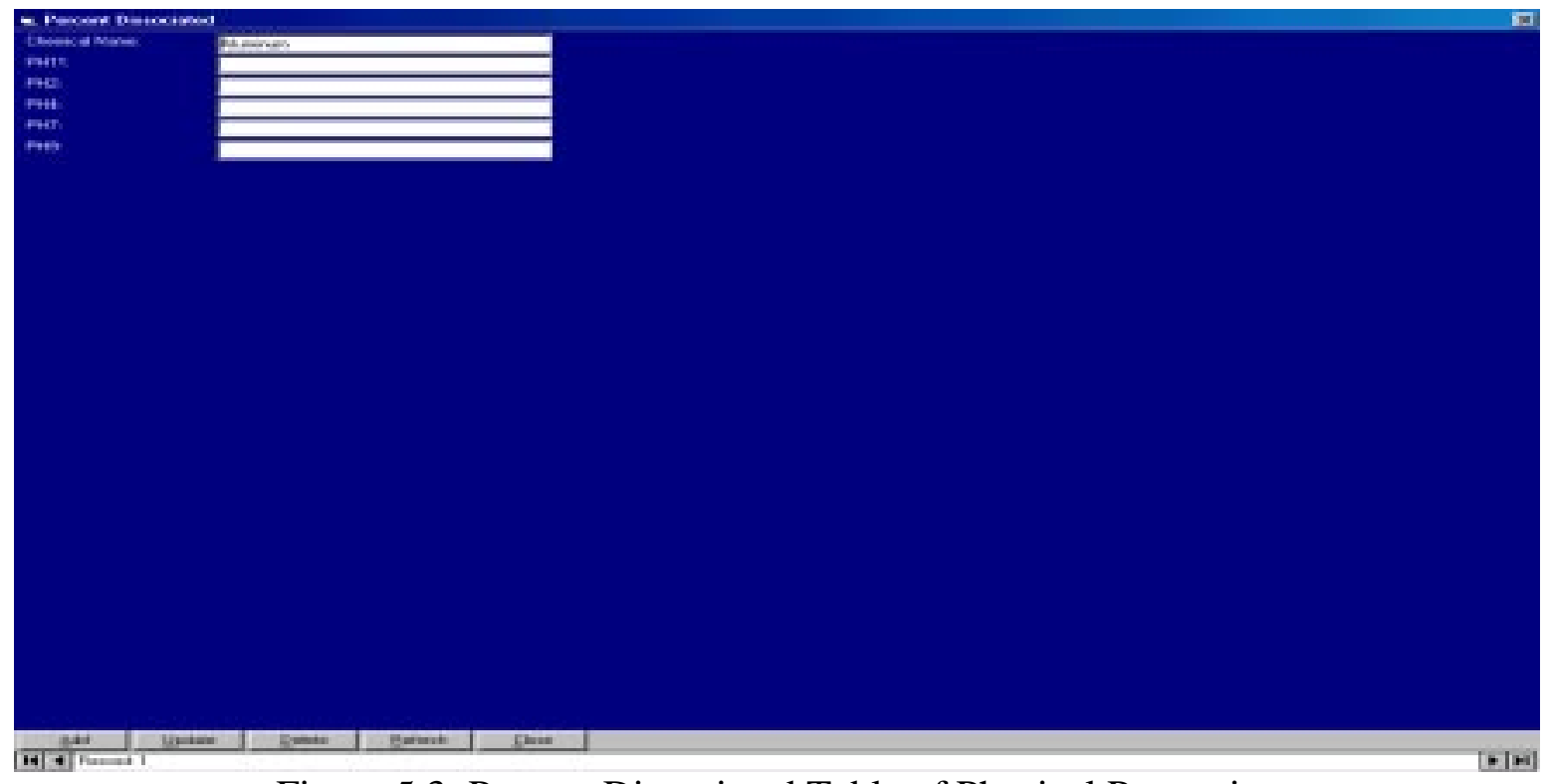

Figure 5.3: Percent Dissociated Table of Physical Properties 
Chemical properties: These can be obtained from the Flammability/Combustibility, Extinguishing Agents, Reactivity/Instability and Corrosivity Tables. Since it is a metal, cadmium doesn't have a flash point, auto ignition temperature and upper and lower exposure limits. It is not corrosive.

When the user clicks on the menu item Flammability/Combustibility of the Physical Properties, the form shown in Figure 5.4 is displayed

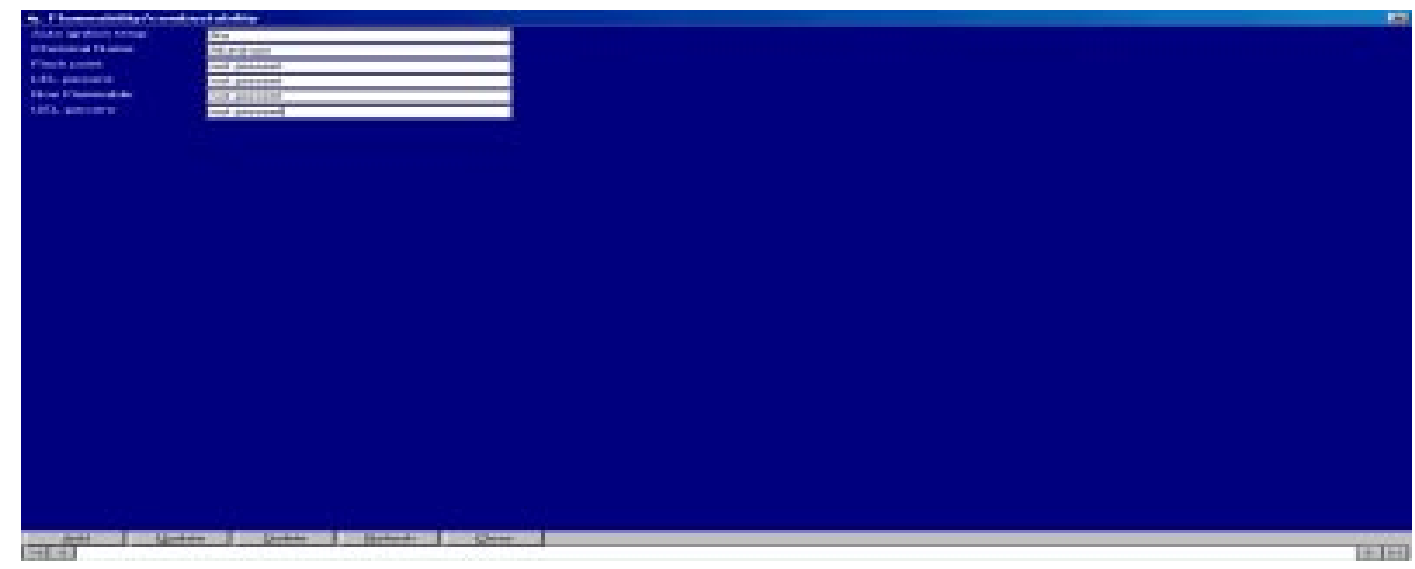

Figure 5.4: showing Flammability/Combustibility Table

When the user clicks on the menu item Extinguishing Agents of the Chemical Properties, a form as shown in Figure 5.5 below is displayed

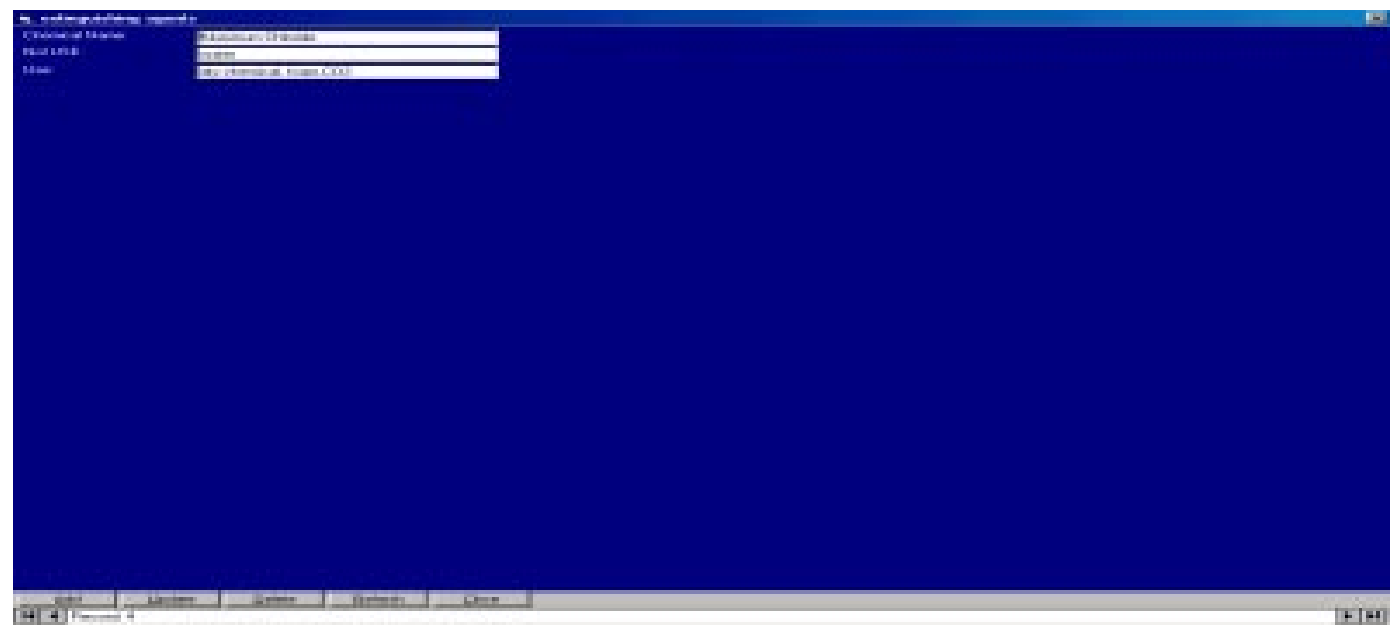

Figure 5.5: Extinguishing Agents Table of Chemical Properties 
When the user clicks on the menu item Reactivity/Instability of the Chemical Properties, form as shown in Figure 5.6 below is displayed

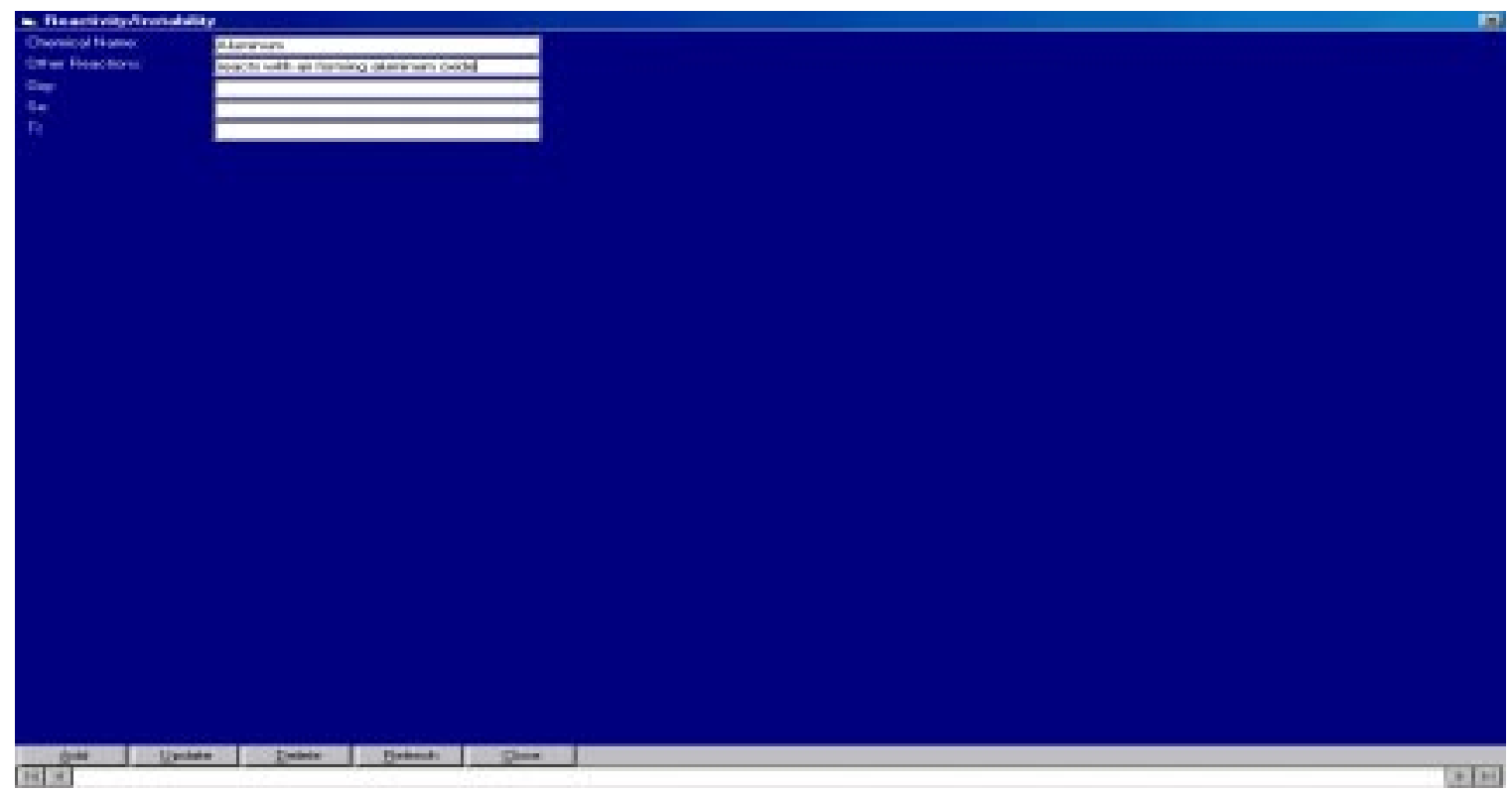

Figure 5.6: Reactivity/Instability Table of Chemical properties

When the user clicks on the menu item Corrosivity of the Chemical Properties, the form as shown in Figure 5.7 below is displayed

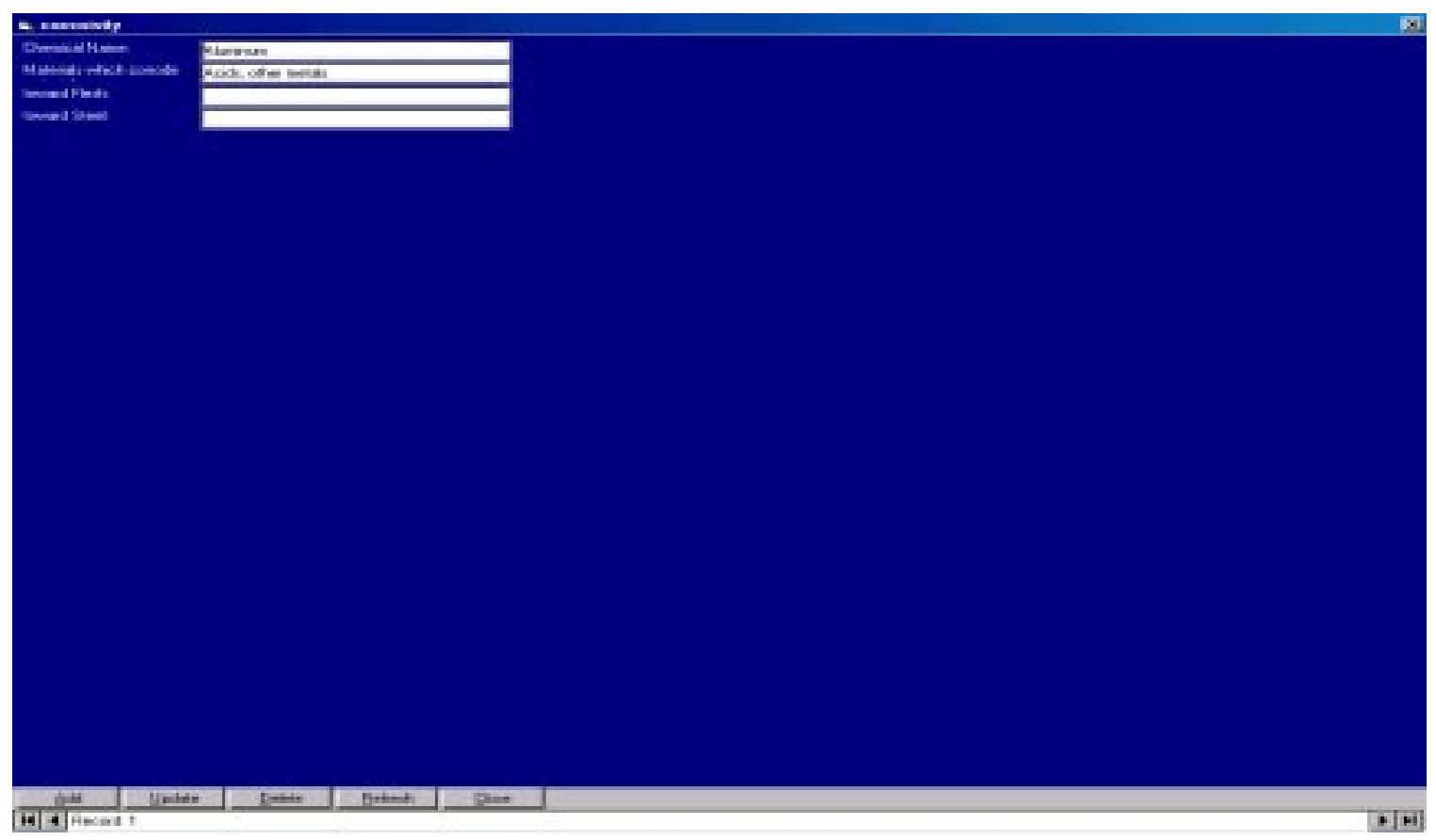

Figure 5.7: Corrosivity Table of chemical Properties 
Health and Ecological Hazards: These can be obtained from Cancer, Aquatic Fate, Acute Symptoms, Chronic Symptoms, Allergen and Oral Acute Aquatic Toxicity tables. Cadmium is genotoxic, has acute symptoms from both inhalation and ingestion, has chronic symptoms both from inhalation and ingestion, and damages the liver badly.

When the user clicks on the menu item Cancer of the Health and Ecological Hazards, the form as shown in Figure 5.8 below is displayed

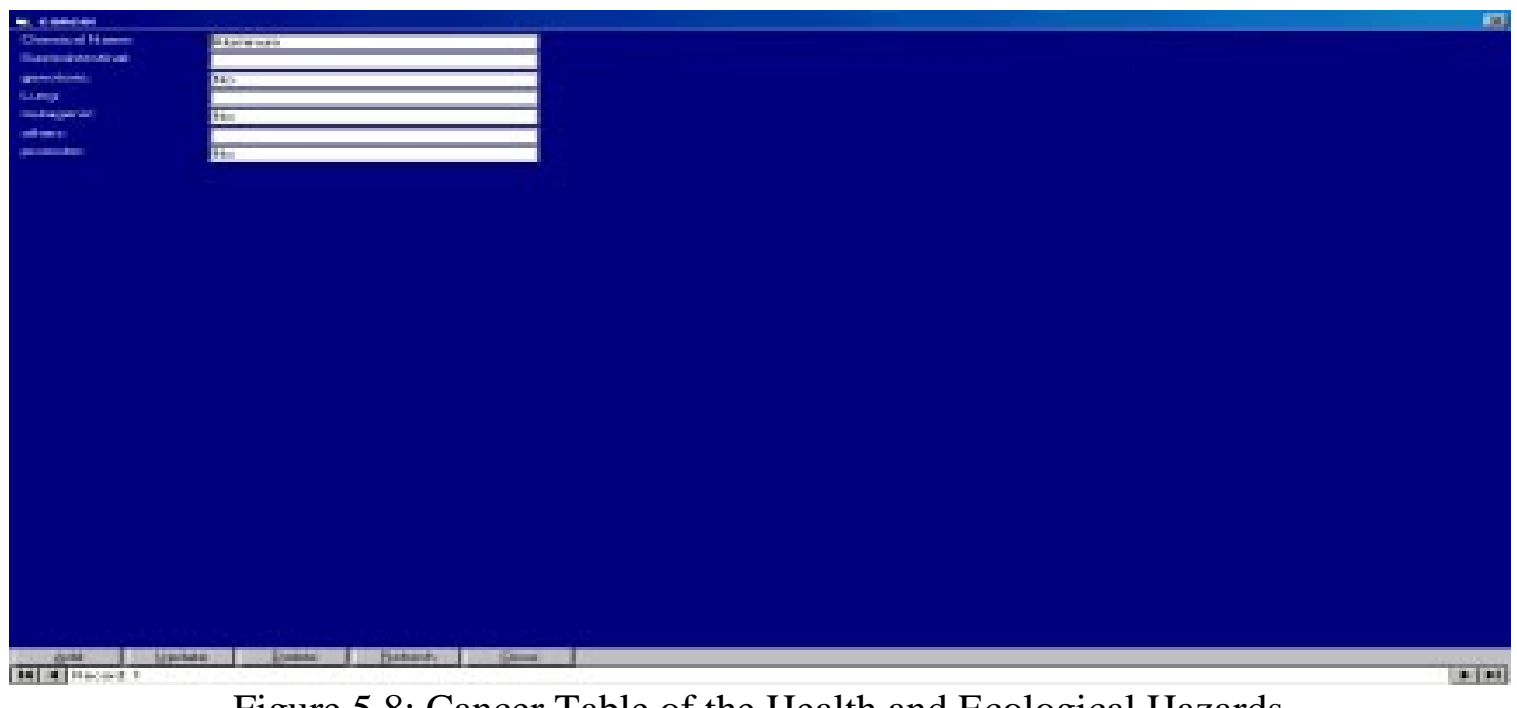

Figure 5.8: Cancer Table of the Health and Ecological Hazards

When the user clicks on the menu item Aquatic Fate of the Health and Ecological Hazards, the form below in Figure 5.9 is displayed

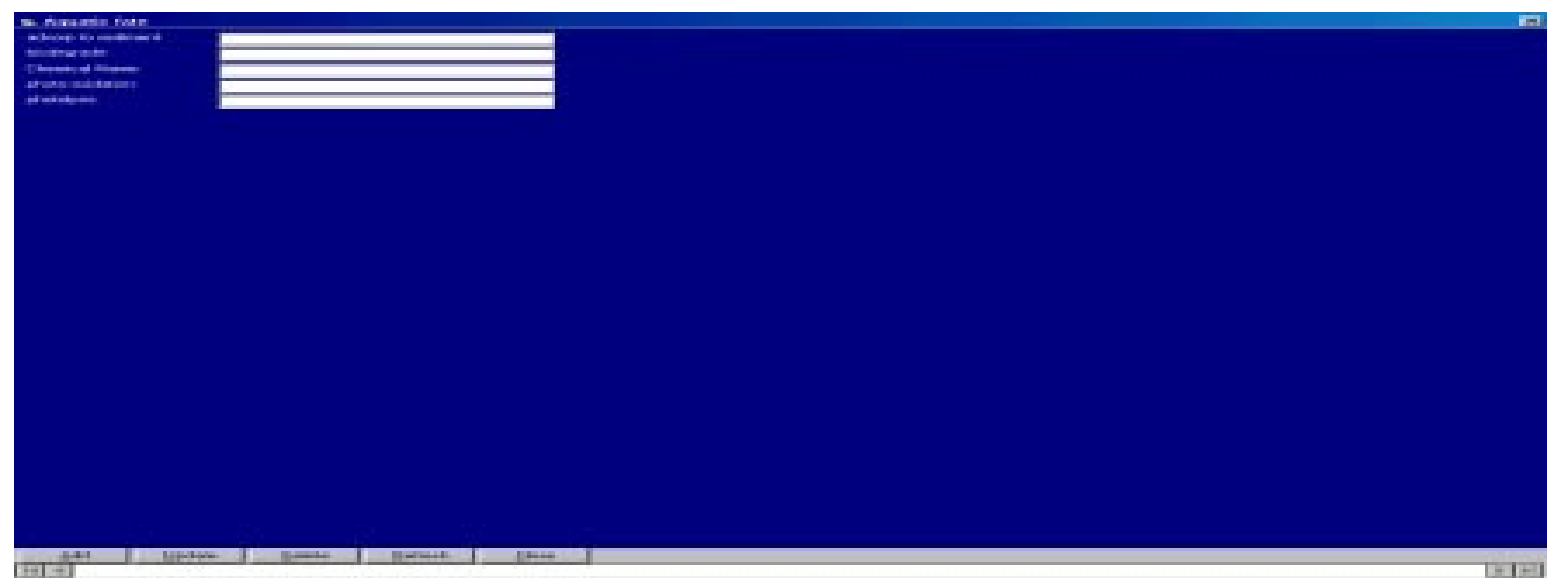

Figure 5.9: Showing the Aquatic Fate Table 
When the user clicks on the menu item acute symptoms of the Health and Ecological Hazards, the form as shown in Figure 5.10 is displayed

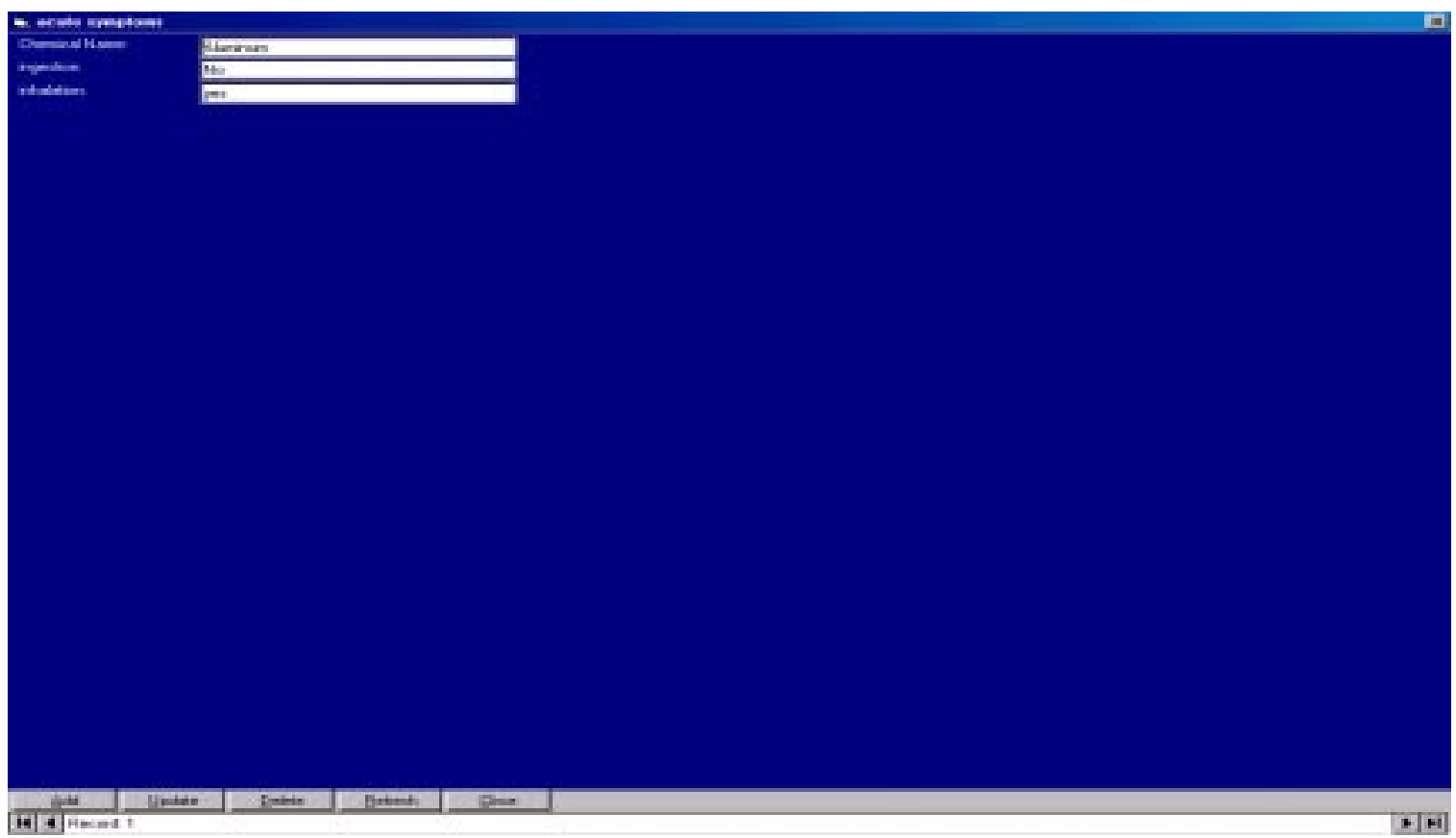

Figure 5.10: Acute Symptoms Table of Health and Ecological Hazards

When the user clicks on the menu item chronic symptoms of the Health and Ecological Hazards, form as shown in Figure 5.11 below is displayed

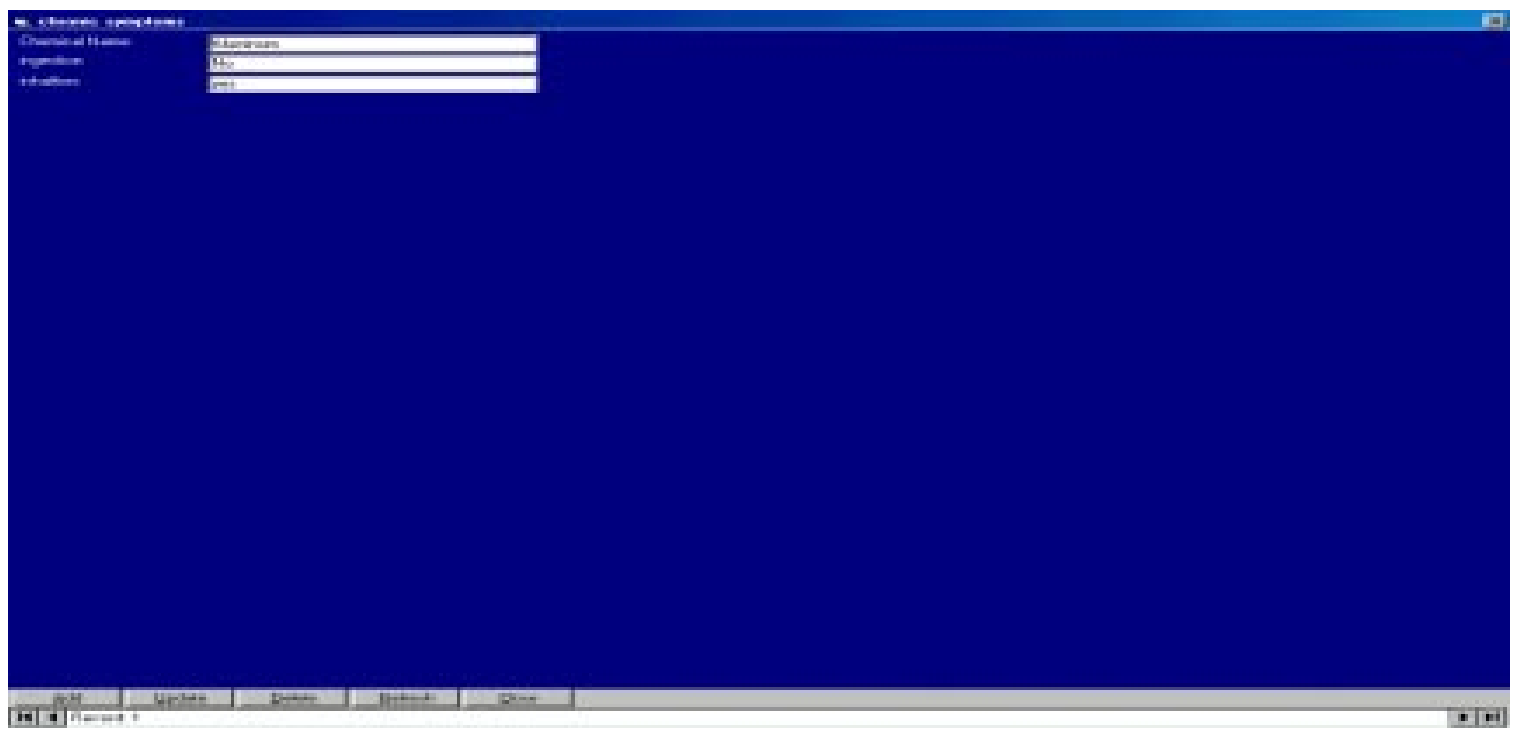

Figure 5.11: Chronic Symptoms Table of Health and Ecological Hazards 
When the user clicks on the menu item Allergen of the Health and Ecological Hazards, the form as shown in Figure 5.12 below is displayed

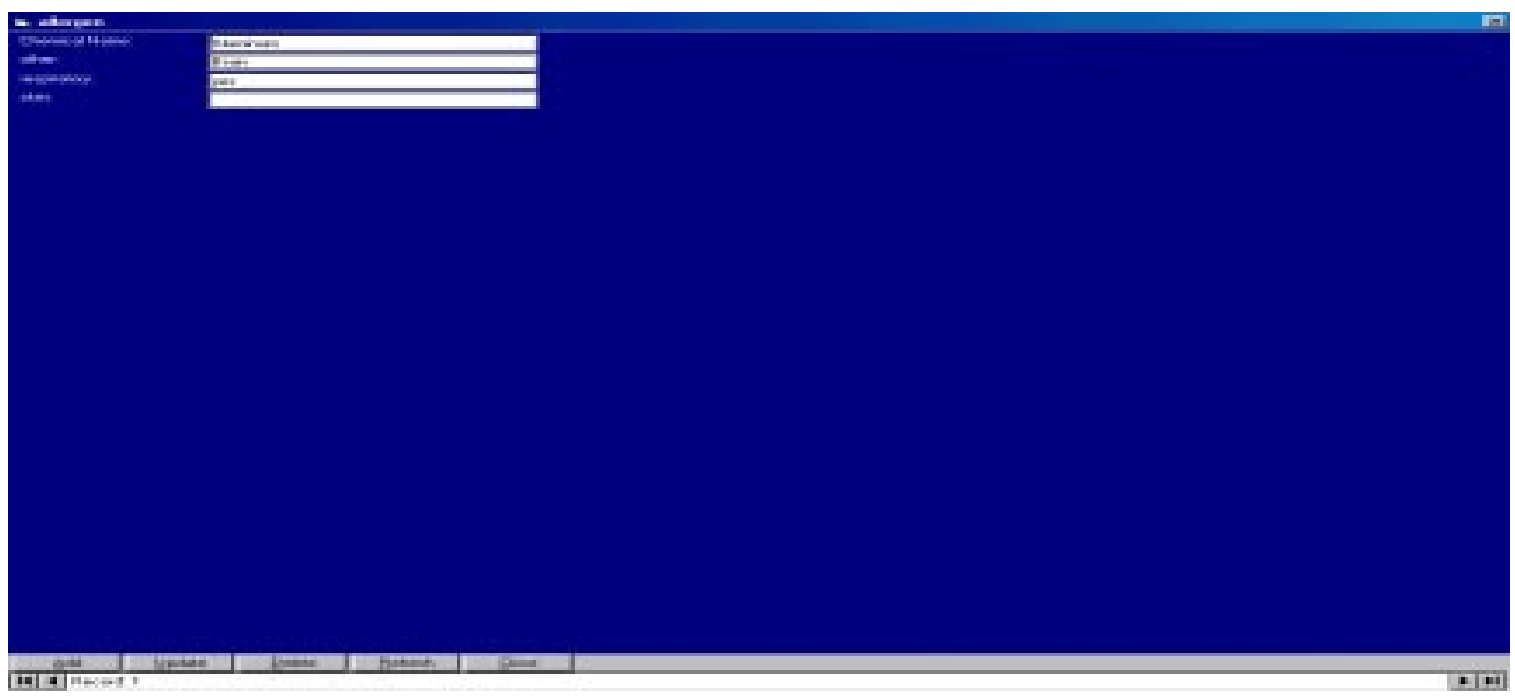

Figure 5.12: Allergen Table of Health and Ecological Hazards

When the user clicks on the menu item Oral Acute Aquatic Toxicity of the Health and Ecological Hazards, the form below in Figure 5.13 is displayed

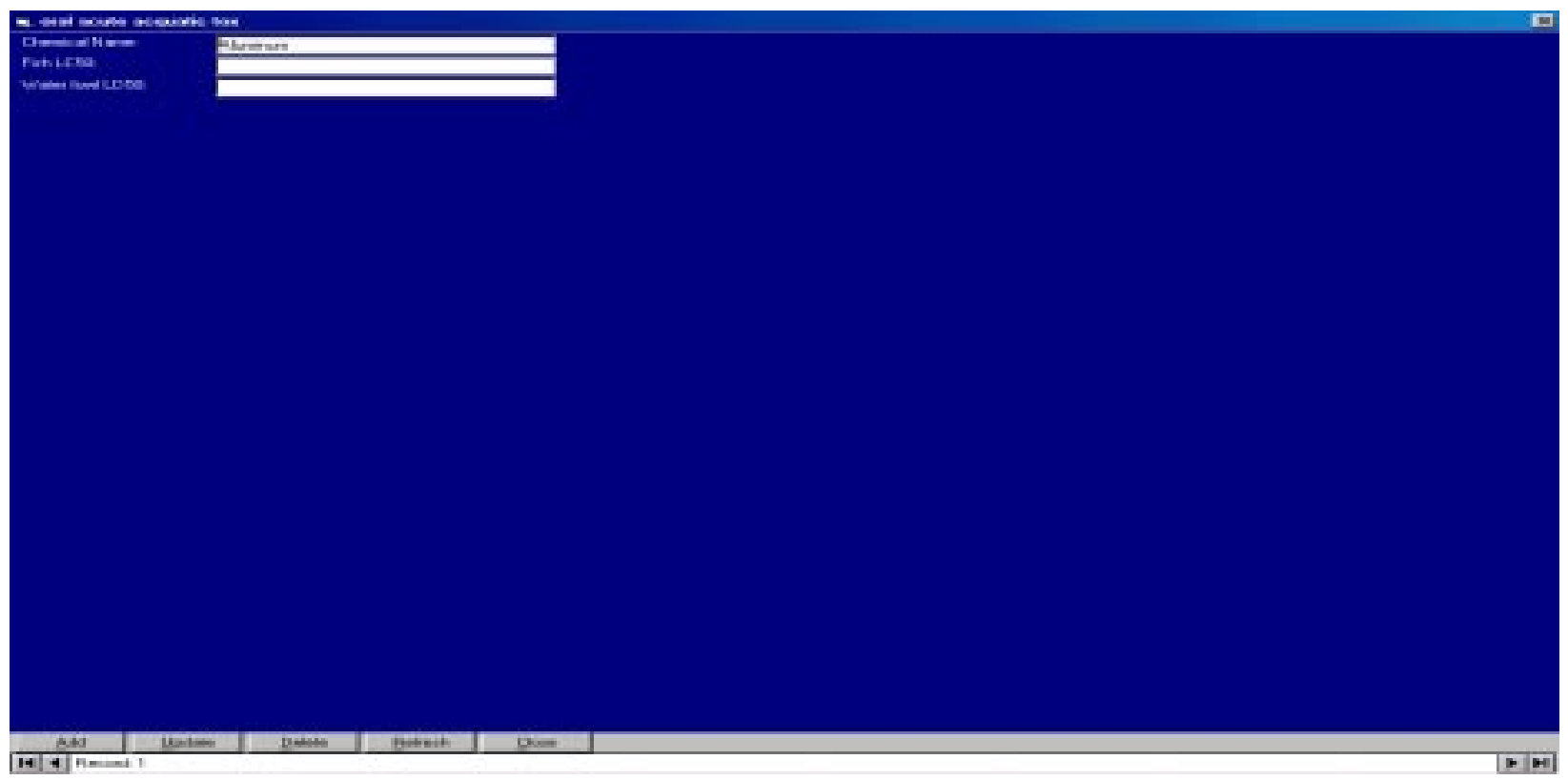

Figure 5.13 - Oral Acute Aquatic Toxicity Table

\section{Safety and Environmental Regulations:}

These can be obtained from Clean Air Act, Clean Water Act, RCRA, and DOT tables. 
When the user clicks on the menu item Clean Air Act of the Safety and Environmental Regulations, the form below in Figure 5.14 is displayed

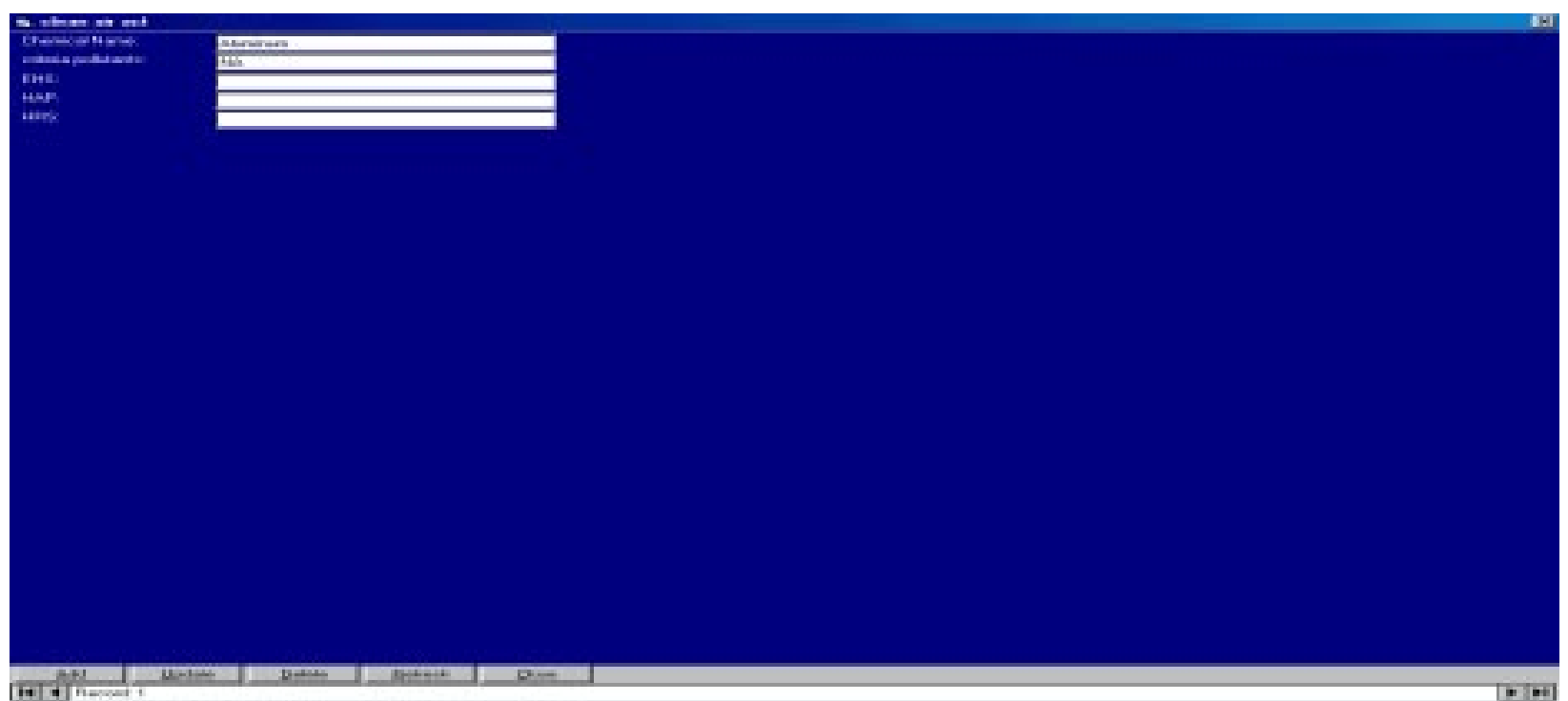

Figure 5.14: Showing the Clean Air Act Table

When the user clicks on the menu item RCRA table of the Safety and Environmental Regulations, the form below in Figure 5.15 is displayed

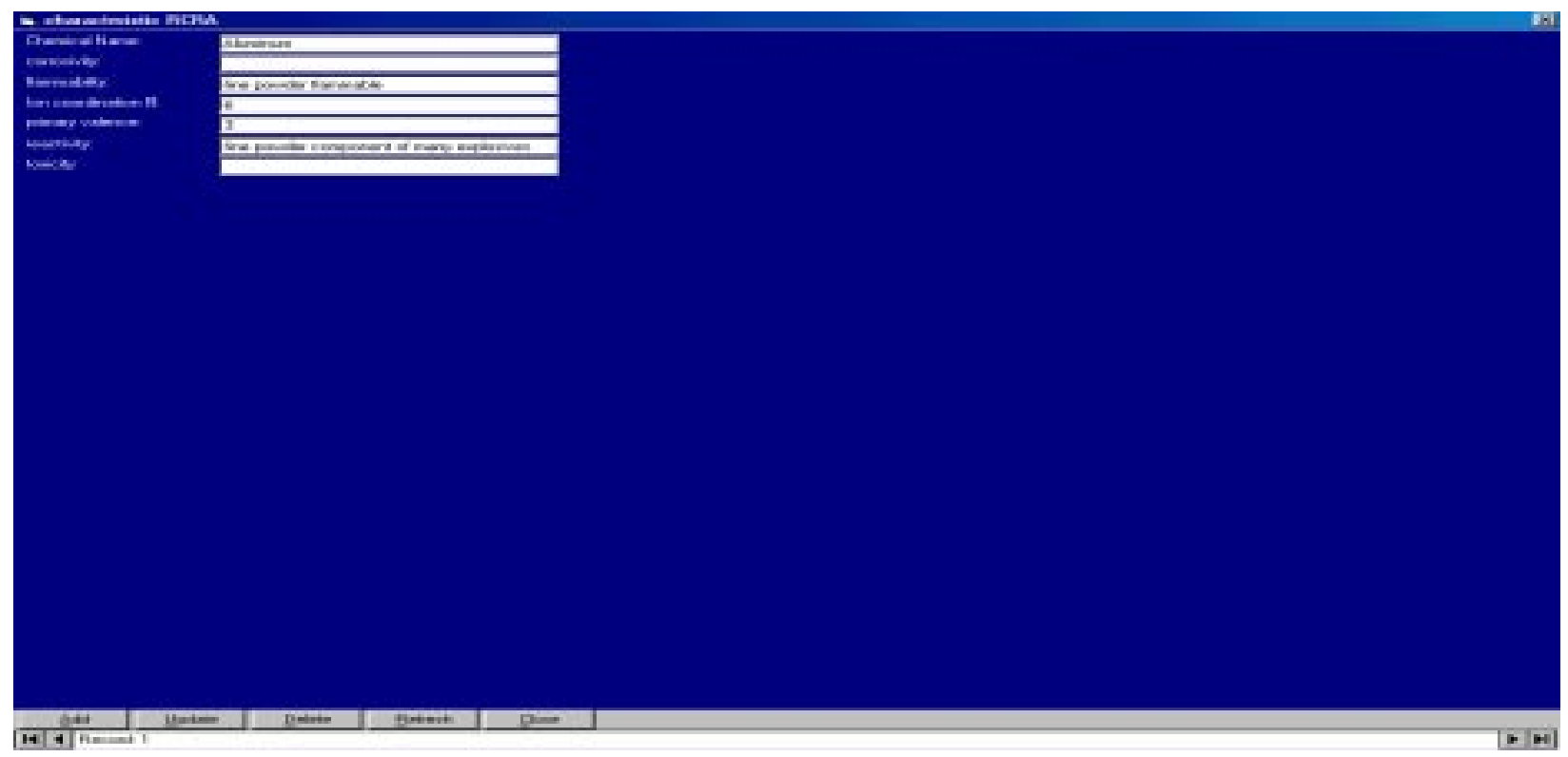

Figure 5.15: Showing the RCRA Table 
When the user clicks on the menu item DOT of the Safety and Environmental Regulations, the form as shown in Figure 5.16 below is displayed

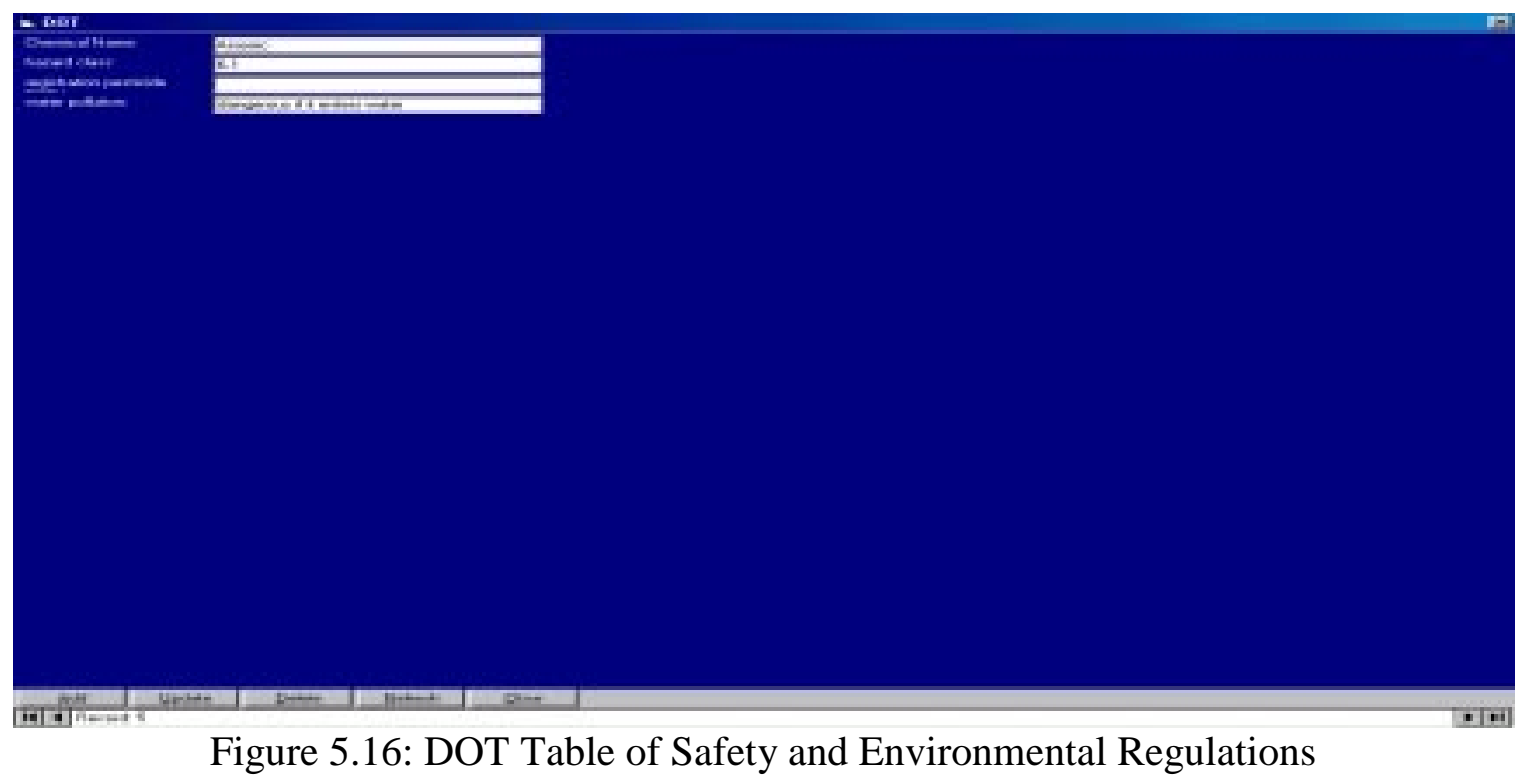

Environmental Concern: These can be obtained from Atmospheric Fate, Terrestrial Fate and BOD tables.

When the user clicks on the menu item Terrestrial Fate of the Environmental Concern, the form as shown in Figure 5.17 below is displayed

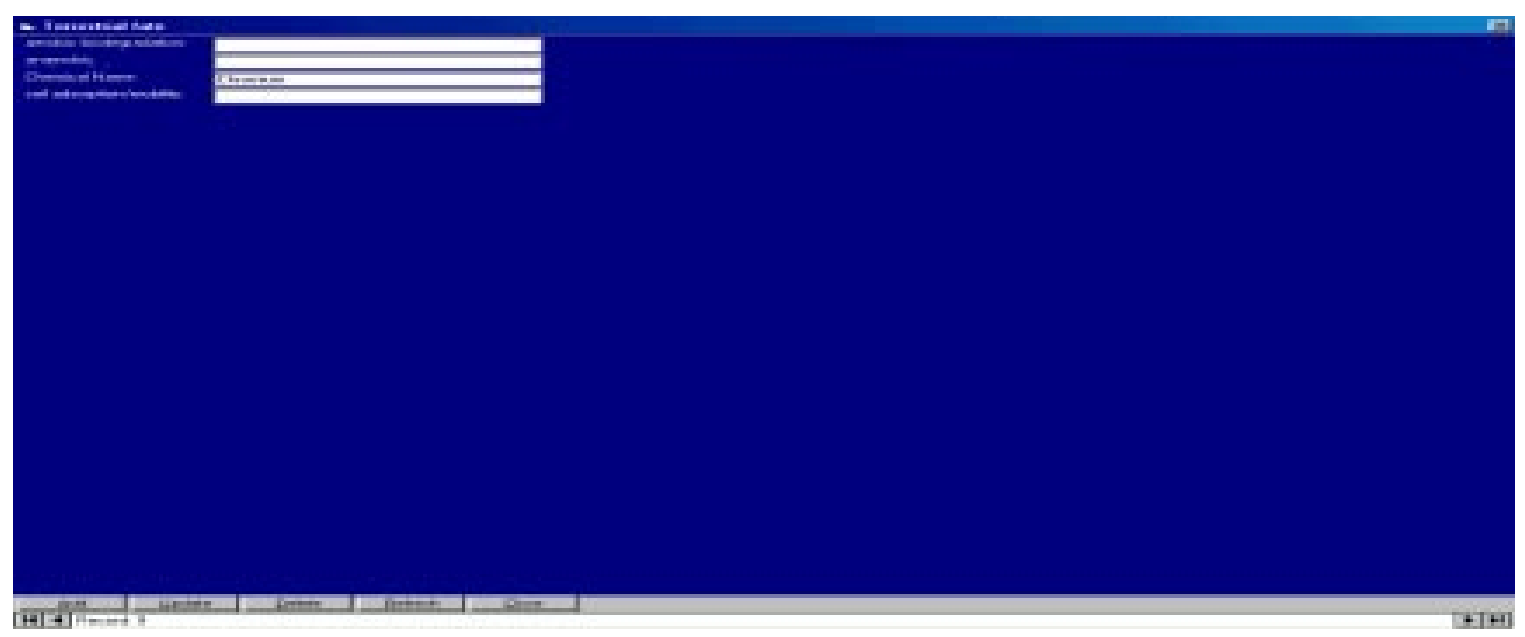

Figure 5.17: Terrestrial Fate Table 
When the user clicks on the menu item Atmospheric Fate of the Environmental Concern menu selection the form as shown in Figure 5.18 below is displayed

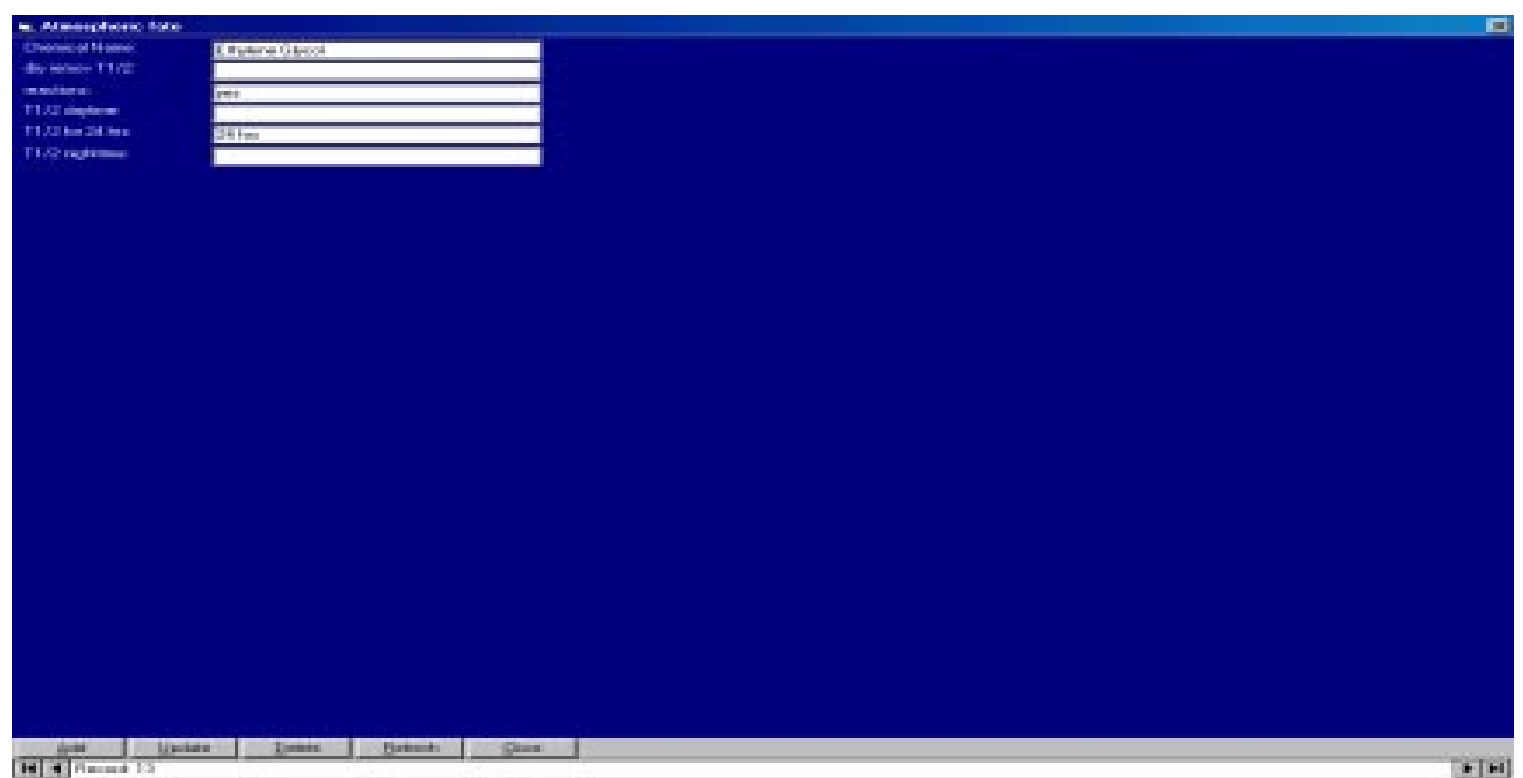

Figure 5.18: Atmospheric Fate Table

When the user clicks on the menu item BOD of the Environmental Concern pull, the form below in Figure 5.19 is displayed

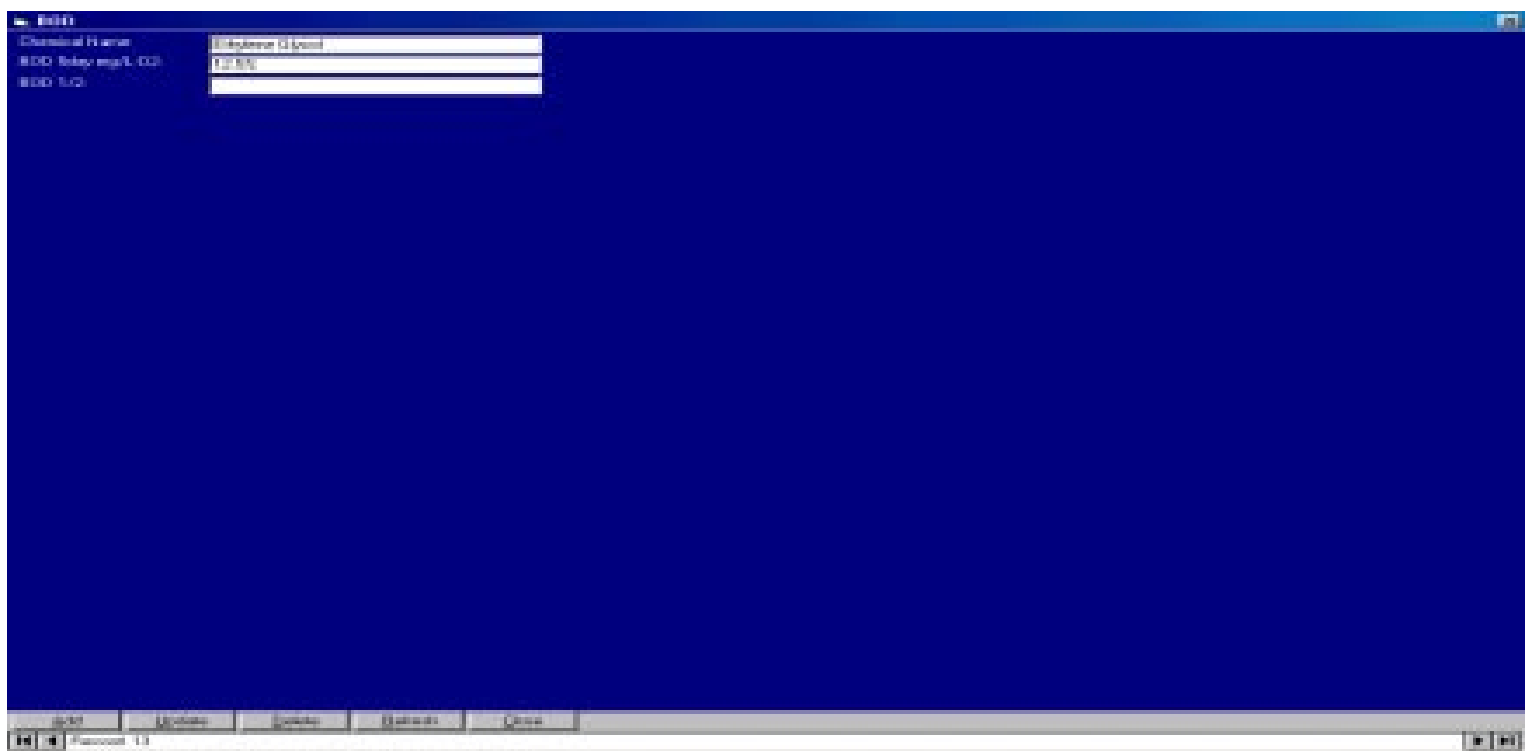

Figure 5.19: BOD Table 
Exposure Criteria: These can be obtained from Worker Exposure Criteria (acute- STEL), Worker Exposure Criteria (acute-CEIL) and Worker Exposure Criteria (chronic-TWA) tables. Cadmium has a TWA TLV of $0.05 \mathrm{mg} / \mathrm{m}^{3}$ and TWA PEL of $0.3 \mathrm{mg} / \mathrm{m}^{3}$.

When the user clicks on the menu item Worker Exposure Criteria (acute-STEL) of the Exposure Criteria, the form as shown in Figure 5.20 below is displayed

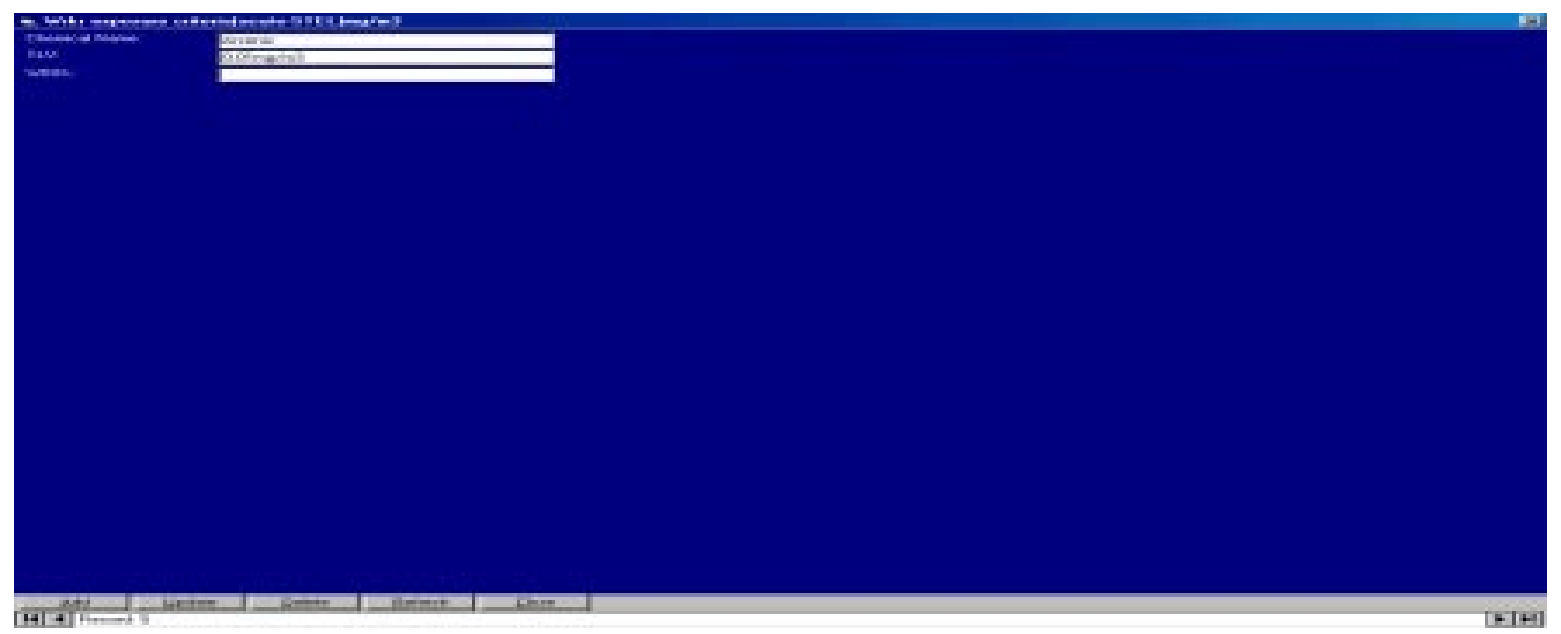

Figure 5.20: Worker Exposure Criteria (acute-STEL) Table

When the user clicks on the menu item Worker Exposure Criteria (acute-CEIL) of the Exposure Criteria, the form below in Figure 5.21 is displayed

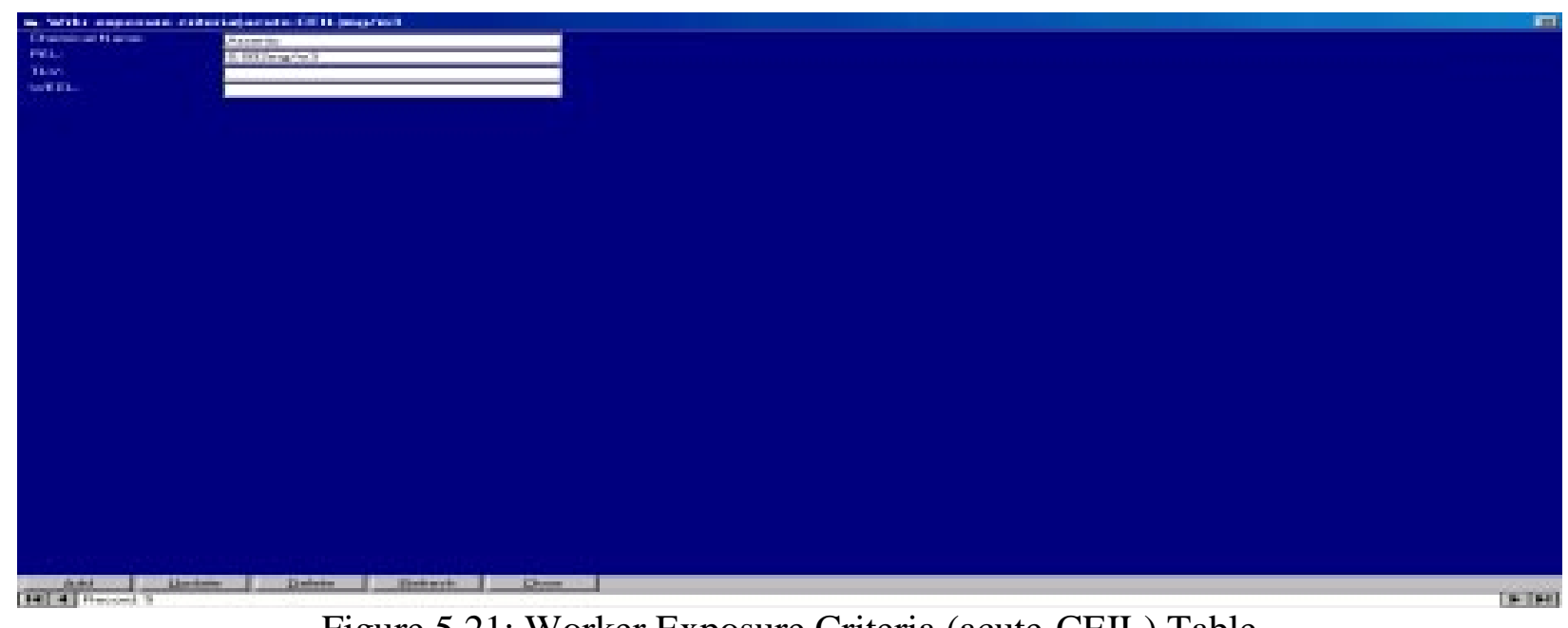

Figure 5.21: Worker Exposure Criteria (acute-CEIL) Table 
When the user clicks on the menu item Worker Exposure Criteria (chronic-TWA) of the Exposure Criteria, the form below in Figure 5.22 is displayed.

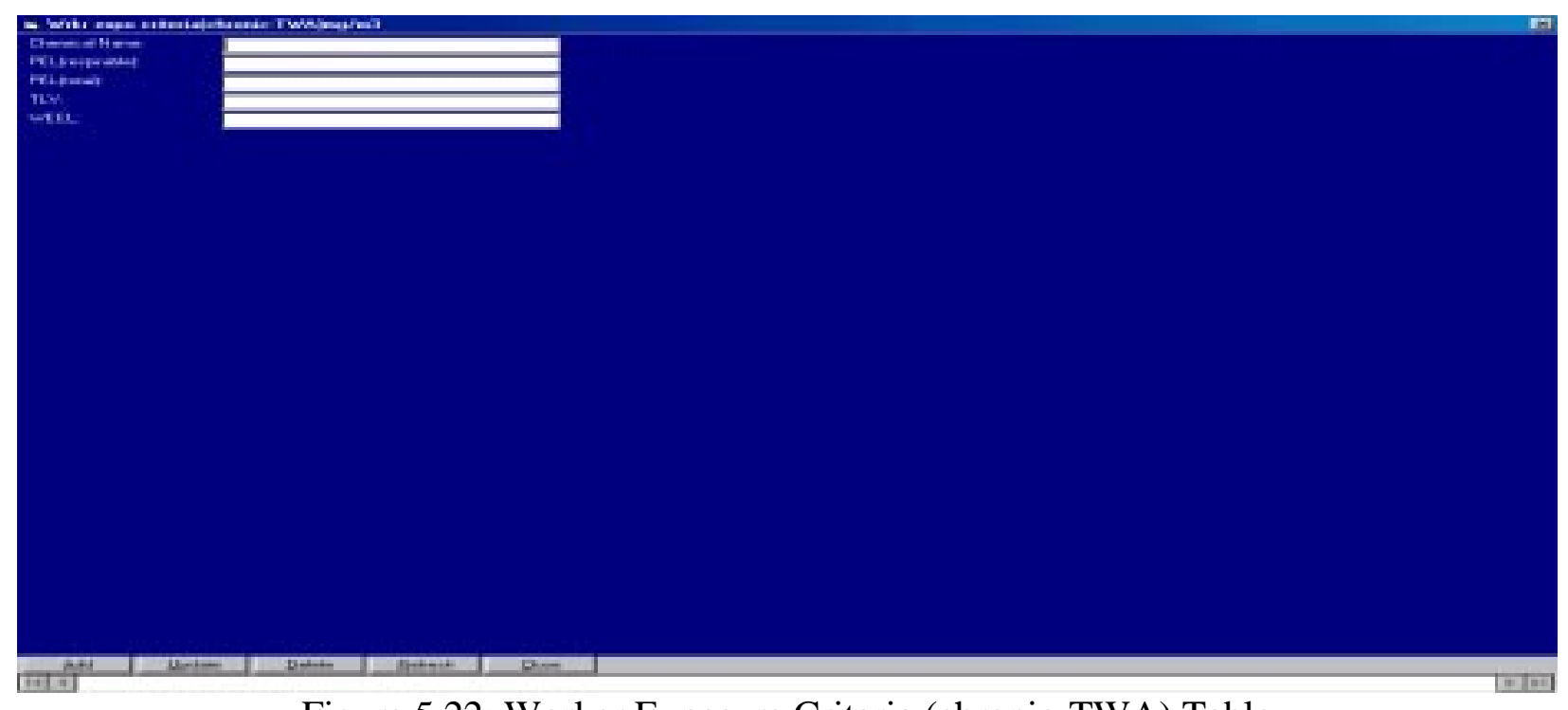

Figure 5.22: Worker Exposure Criteria (chronic-TWA) Table

When the user clicks on the menu item Search, of the Tools a form is displayed with the following three options
j) By CAS\#
k) By Chemical Name
1) By Molecular Formula as shown in Figure 5.23 below

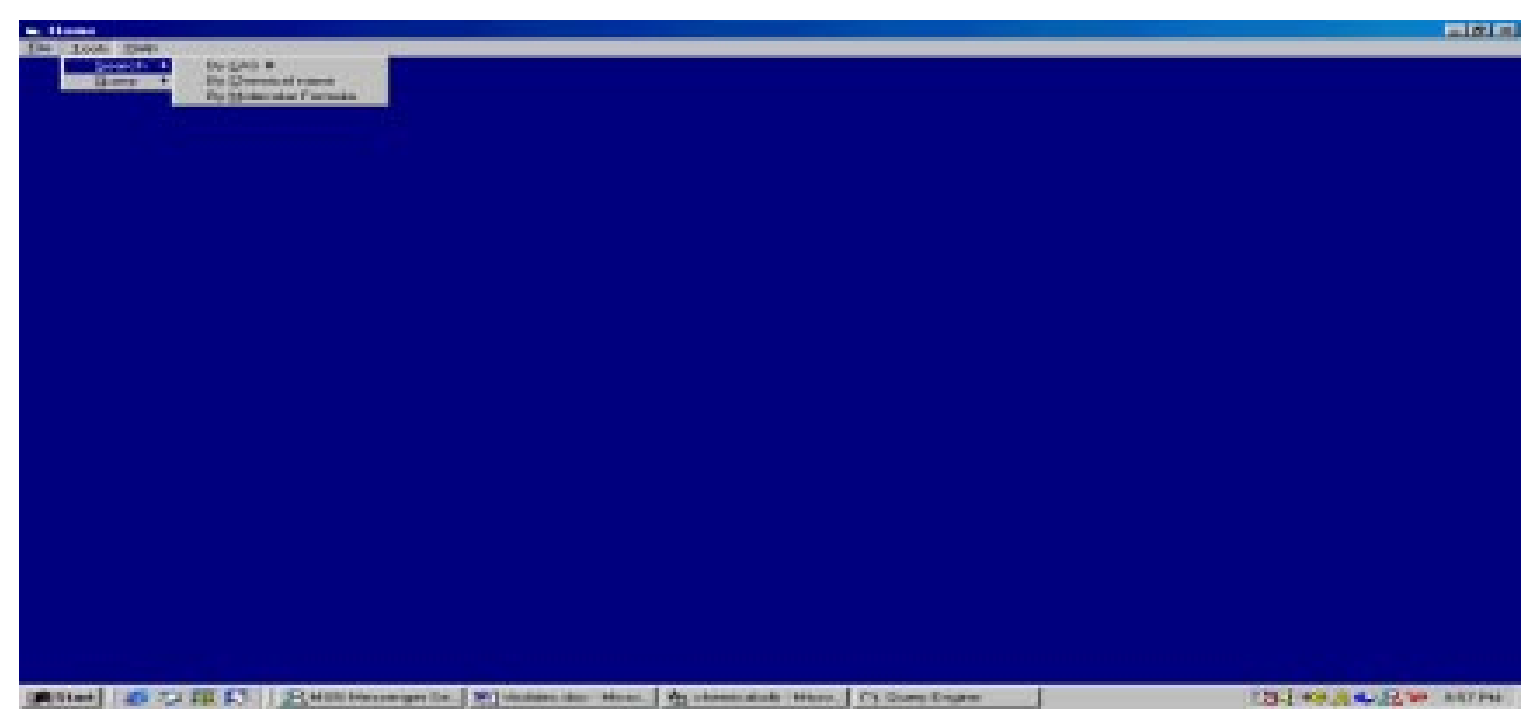

Figure 5.23: Pull down menu for Search 
The user can get specific information desired such as, he can search for a specific Chemical and its properties from the database by inputting the CAS \# or Molecular Formula and vice versa.

When the user clicks on "By CAS\#" in the Search the form shown below opens. A drop down list of all CAS numbers in the database is provided at "Enter the CAS\#" in the form at the top. User can chose any CAS\# from the drop down list and get the corresponding properties of that particular chemical. The form is as shown in Figure 5.24 below

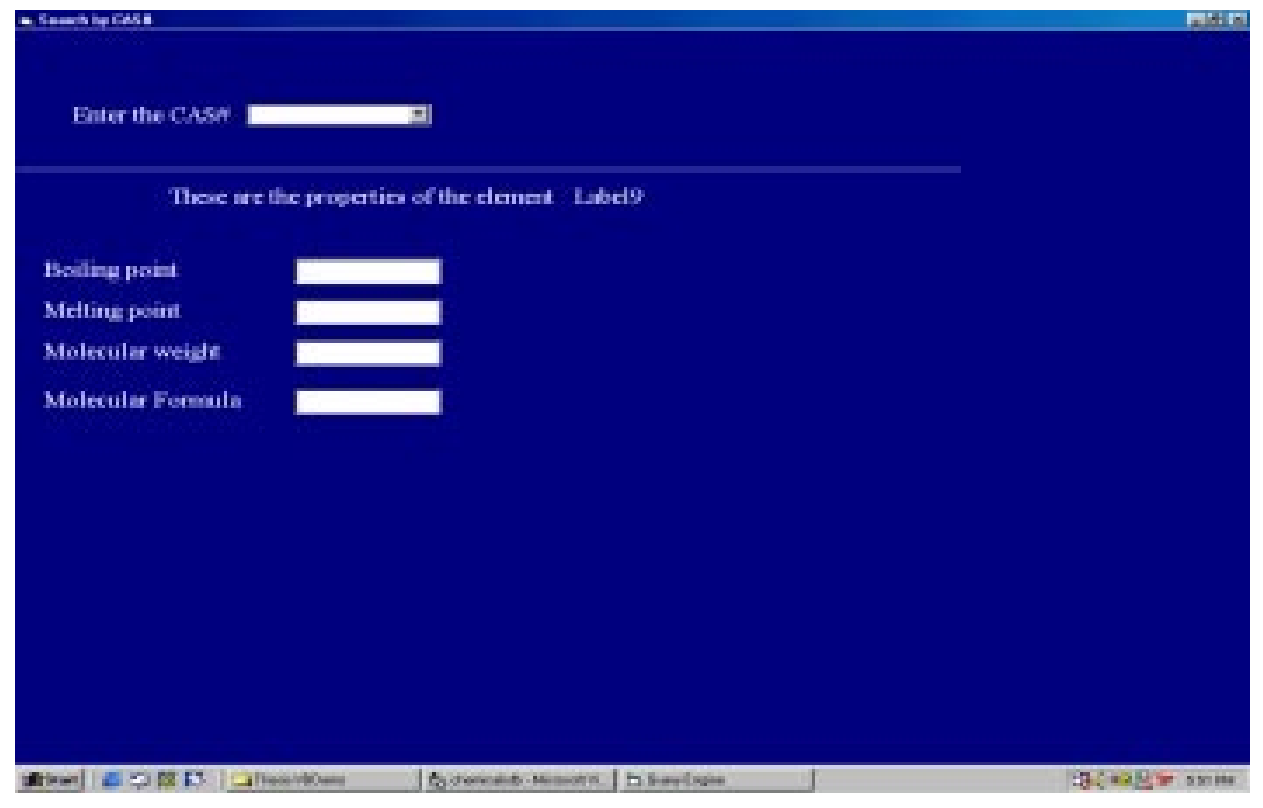

Figure 5.24: Form to enter CAS\#

Let's say the user chose '7429-30-5' from the drop down list of CAS numbers, the search program will search the database and gets the corresponding chemical name and its properties as shown in Figure 5.25 below 


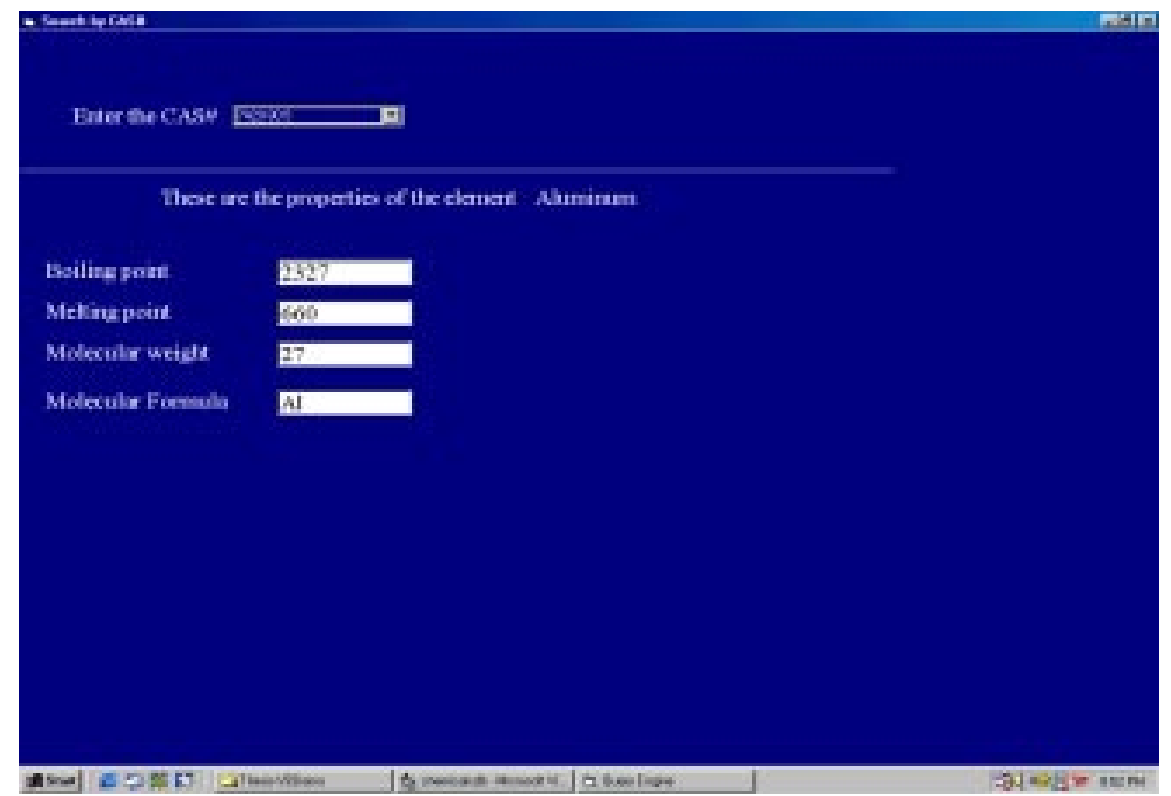

Figure 5.25: Chemical Name and its Properties

When the user clicks on "By Chemical Name" in the Search, a form shown below opens Figure 5.26. A drop down list of all chemical Names in the database is provided at "Enter the Chemical name" in the form at the top. User can chose any Chemical from the drop down list and get the corresponding properties of that particular chemical along with its CAS\#.

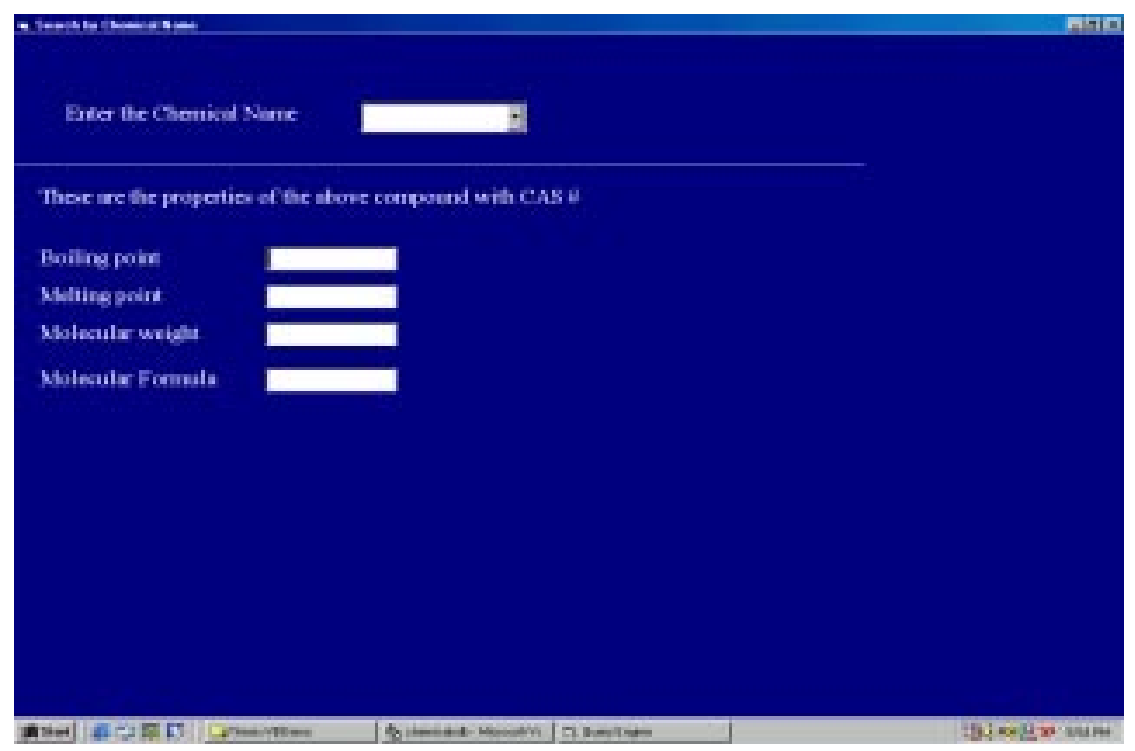

Figure 5.26: Search by Chemical Name 
Let's say the user chose 'Ethylene Glycol', the search program will search the database and gives the corresponding CAS\# and its properties as shown in Figure 5.27 below

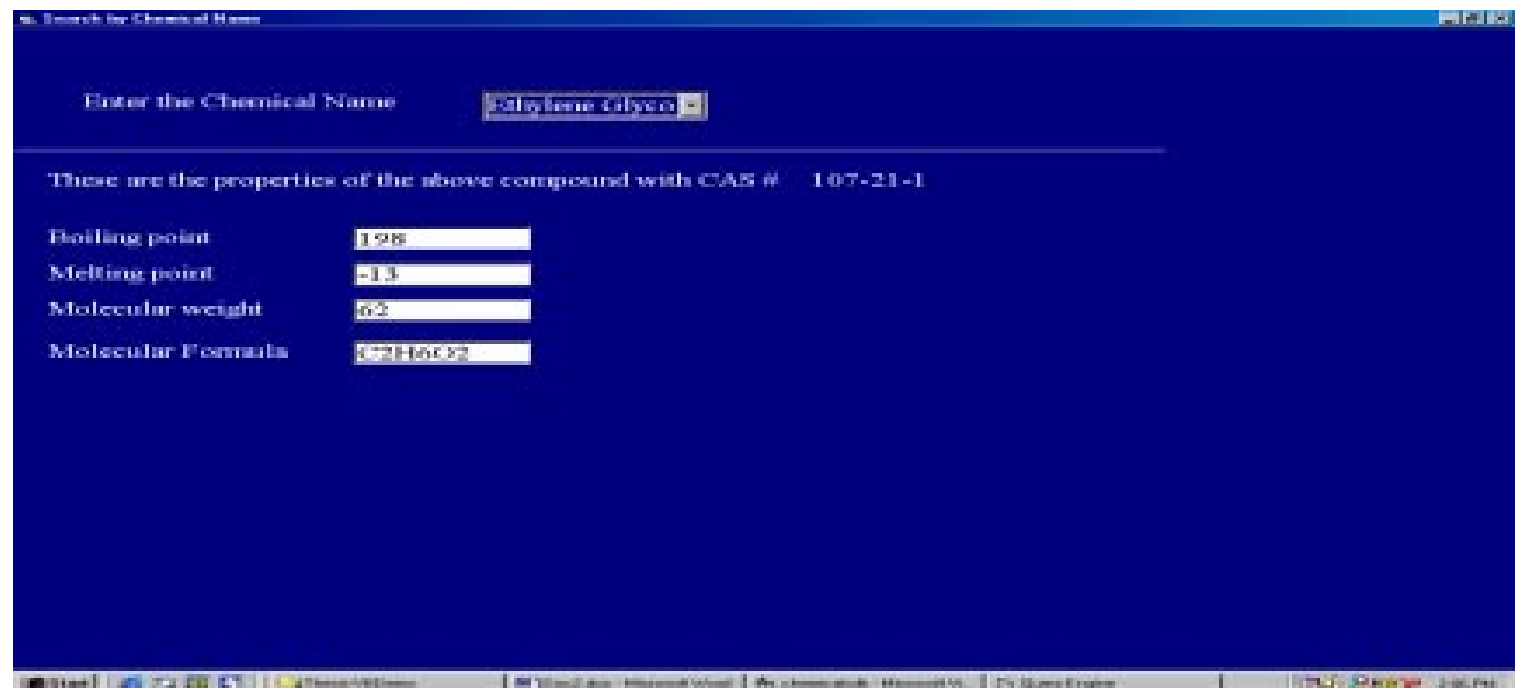

Figure 5.27: Form to choose a Chemical

When the user clicks on "By Molecular Formula" in the Search, the form shown below Figure 5.28 opens. A drop down list of all Molecular Formulas in the database is provided at "Enter the Molecular Formula" in the form at the top. User can chose Molecular Formula of any Chemical from the drop down list and get the corresponding properties of that particular chemical.

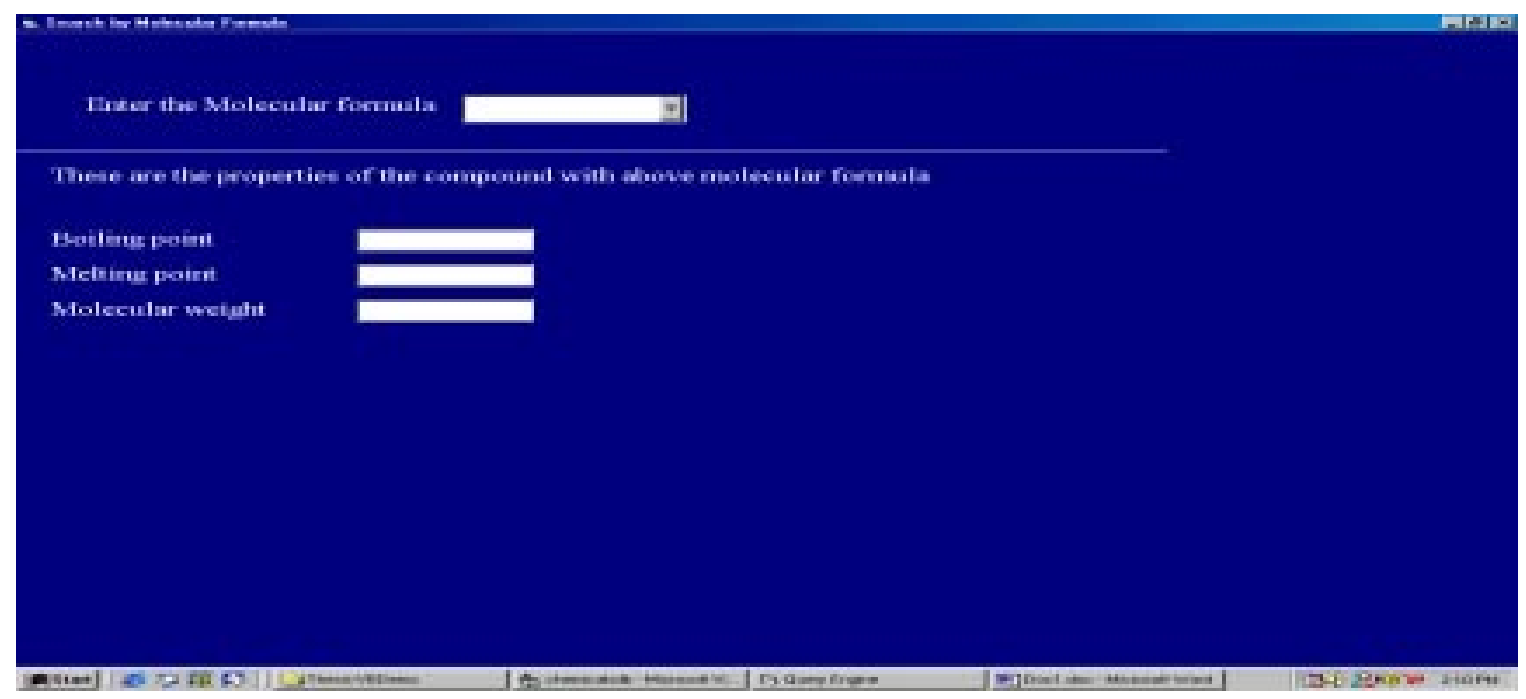

Figure 5.28: Form to Search by Molecular Formula 
Let's say the user chose 'C2H6O2' which is the molecular formula of Ethylene Glycol. The search program will search the database and gives the corresponding chemical name and its properties as shown in Figure 5.29 below

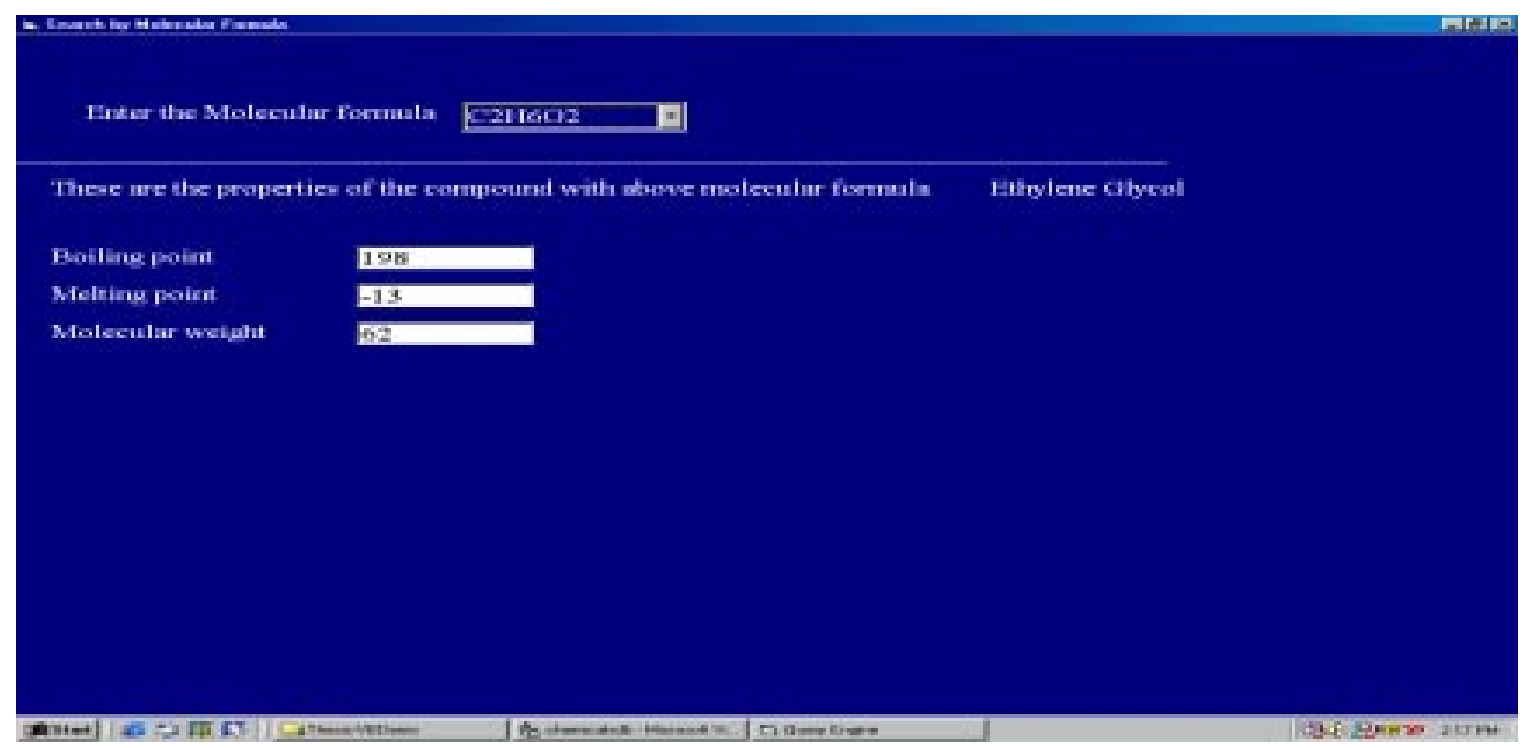

Figure 5.29: Search Results Form

When the user clicks on the menu item Query of the Tools pull down menu the form as in Figure 5.30 shown below is displayed with the following seven options
a) On Molecular Weight
b) On Boiling point
c) On Melting point
d) Molecular formula
e) Save query as and
f) Print 


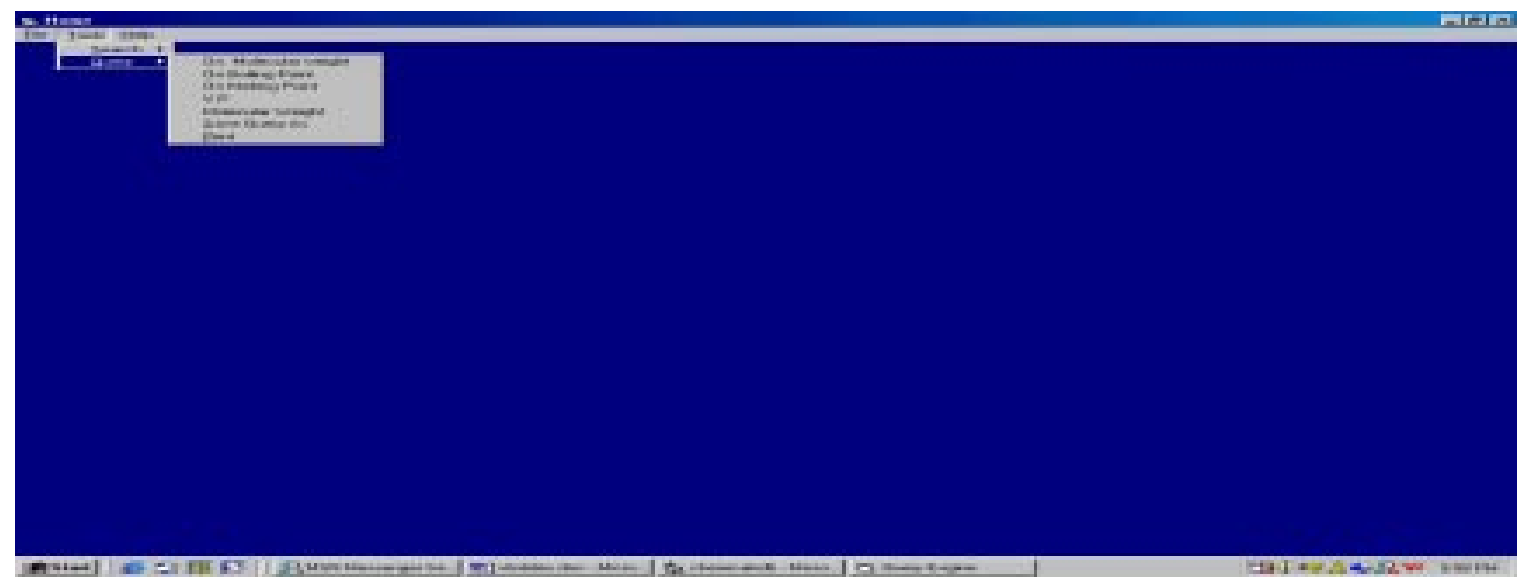

Figure 5.30: Pull Down Menu

Querying with molecular Weight form looks like the Figure 5.31 shown below. This is useful when he wants chemicals and its properties with molecular weight greater or less than certain value.

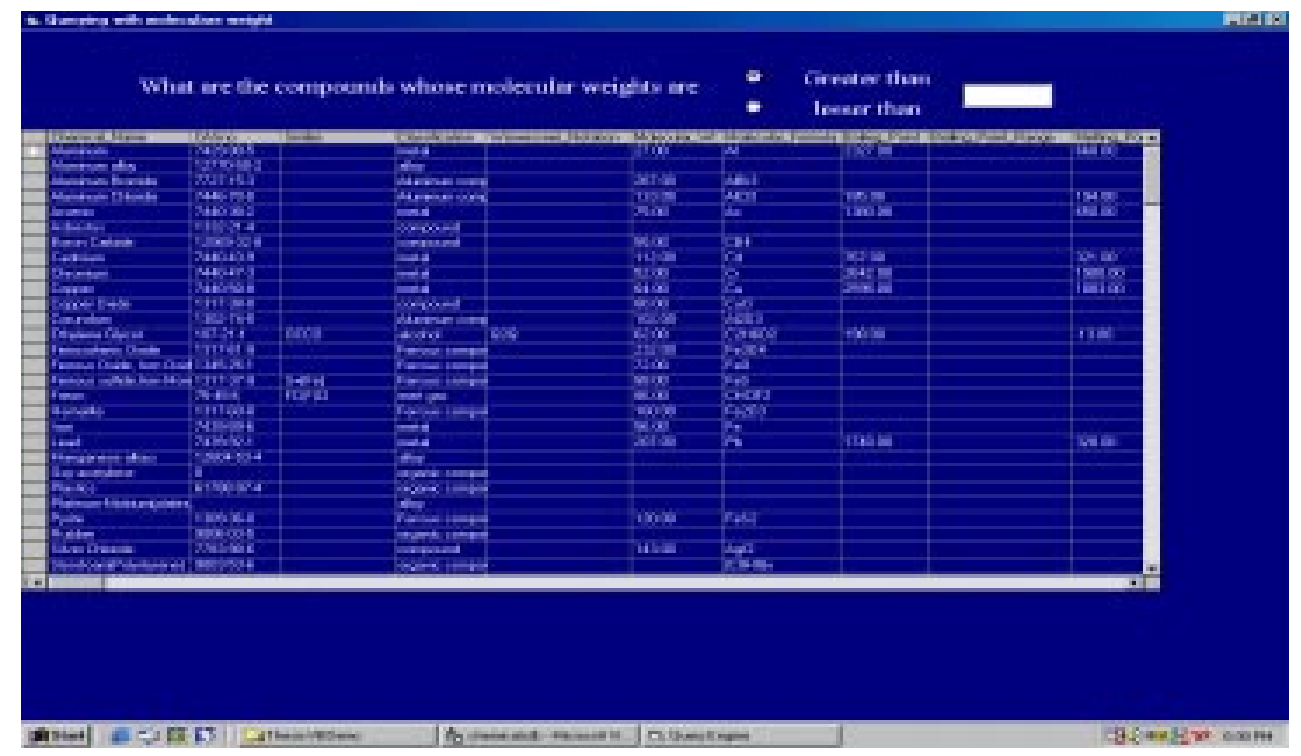

Figure 5.31: Molecular Weight Query

Let's say the user enters a value of 100 and he selects greater than, he can do this as shown below Figure 5.32 


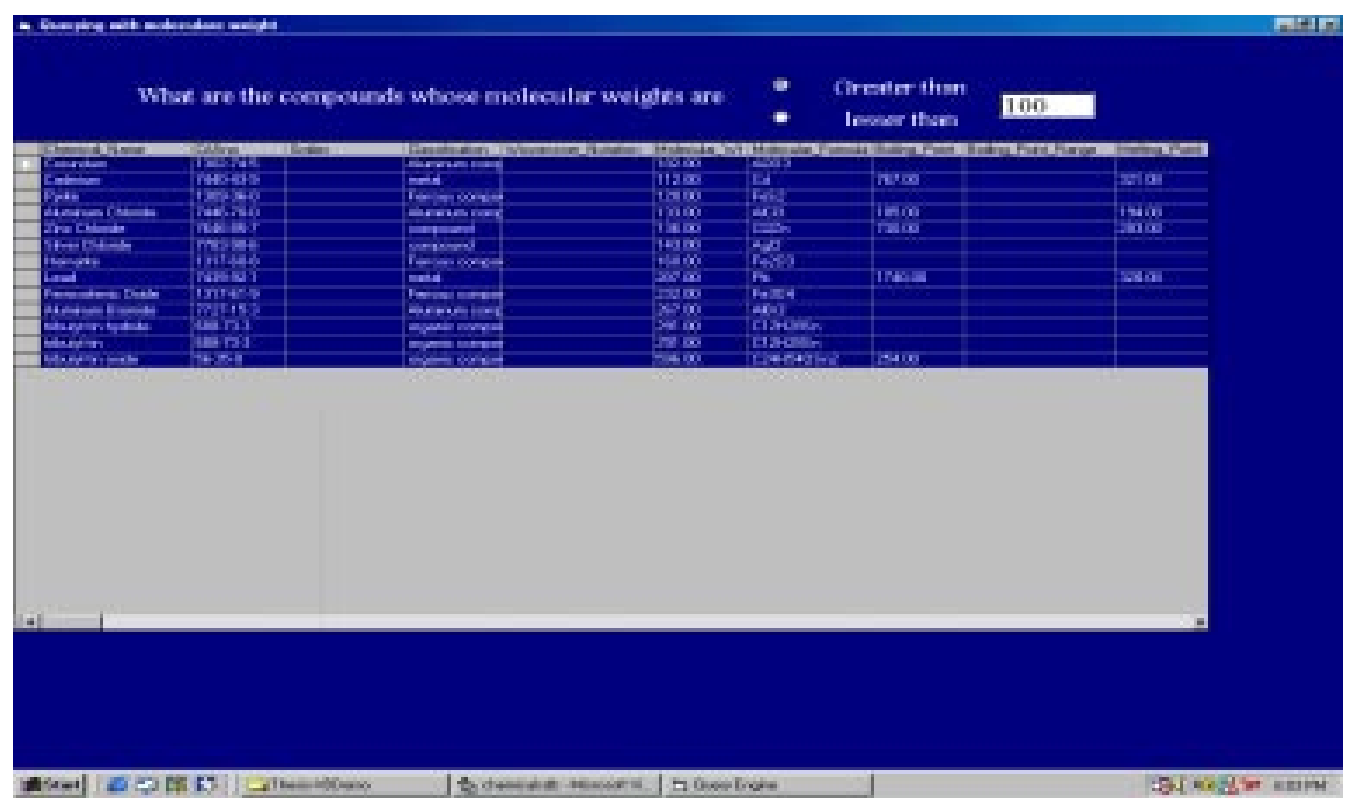

Figure 5.32: Search Criteria Form.

After the selection on clicking Enter all the chemicals with molecular weight greater than 100 and their corresponding properties are displayed. This can be seen from the form below Figure 5.33

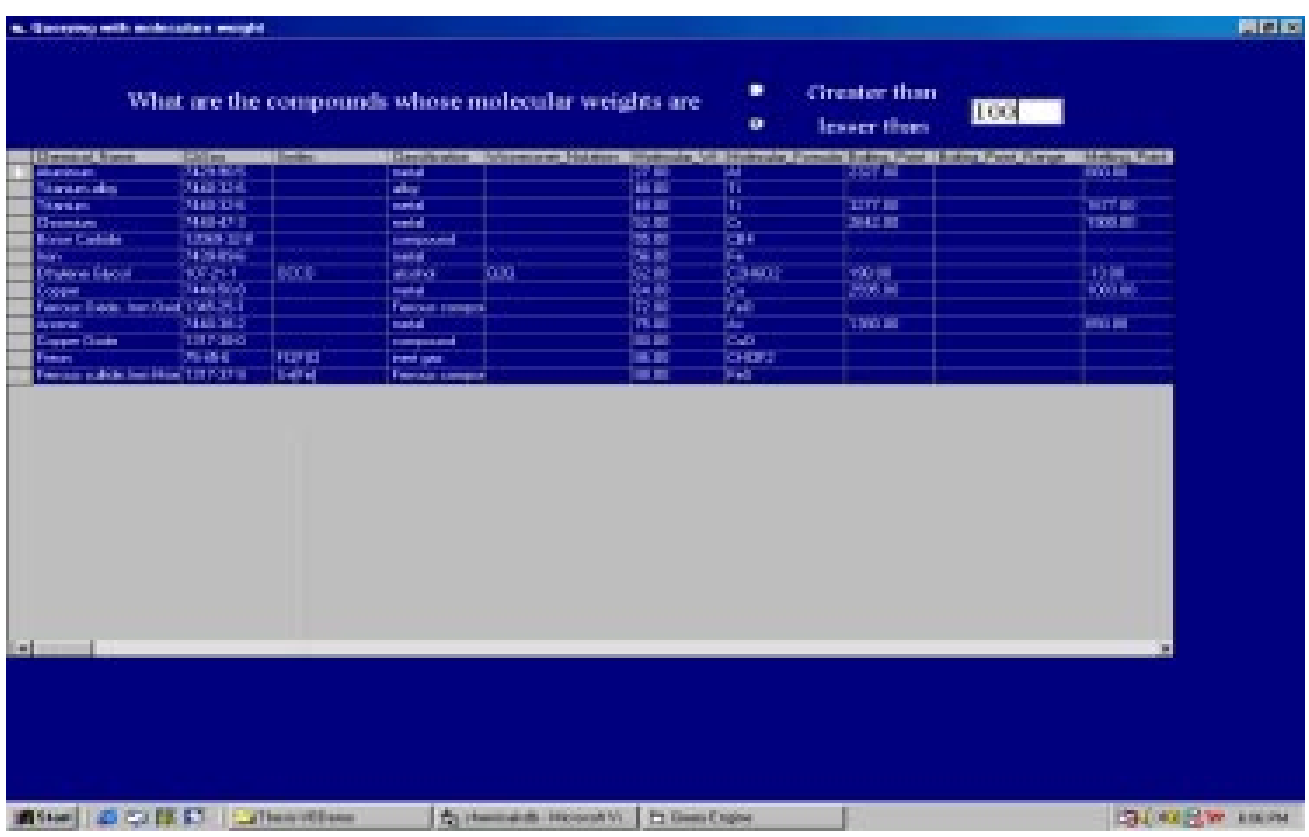

Figure 5.33: Search Results Form 
Note: the query is executed and the results will be displayed only after the user enters the specified value and clicks "Enter"

Querying with Boiling Point form looks like the Figure 5.34 shown below. This is useful when user wants chemicals and its properties with boiling point greater or less than certain value.

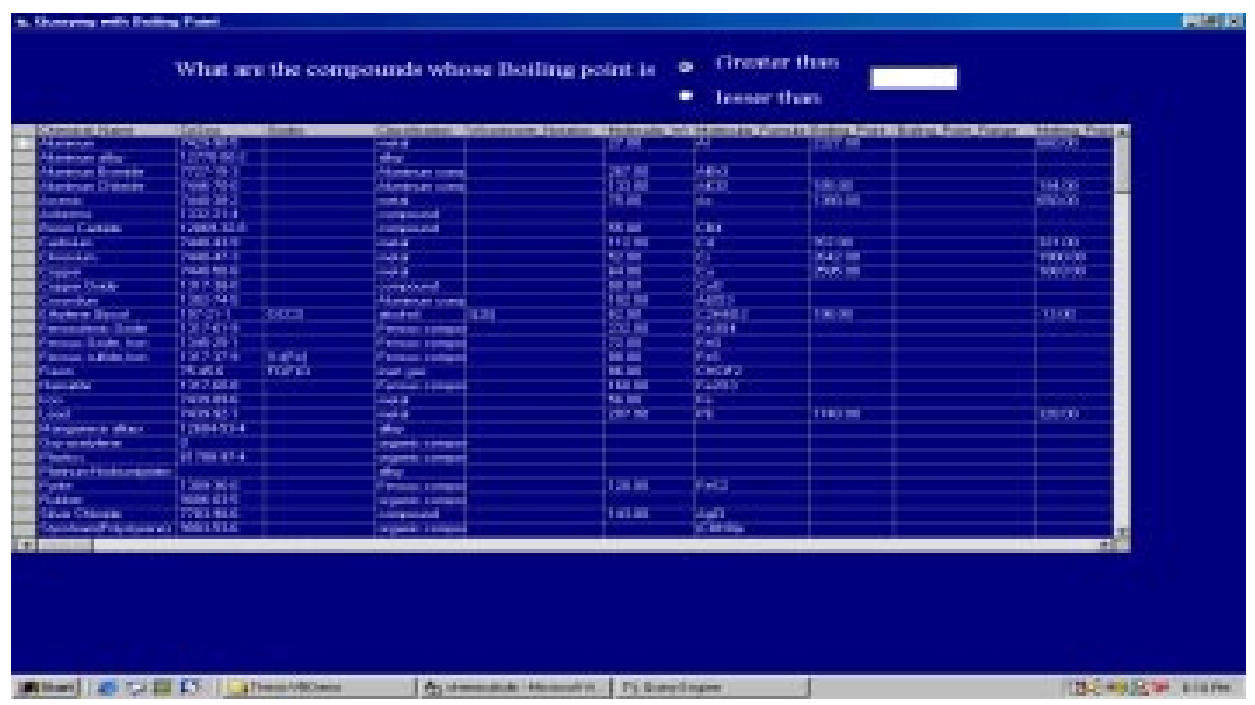

Figure 5.34: Boiling Point Query

Let's say the user enters a value of 1000 and he selects lesser than, as shown below- Figure 5.35

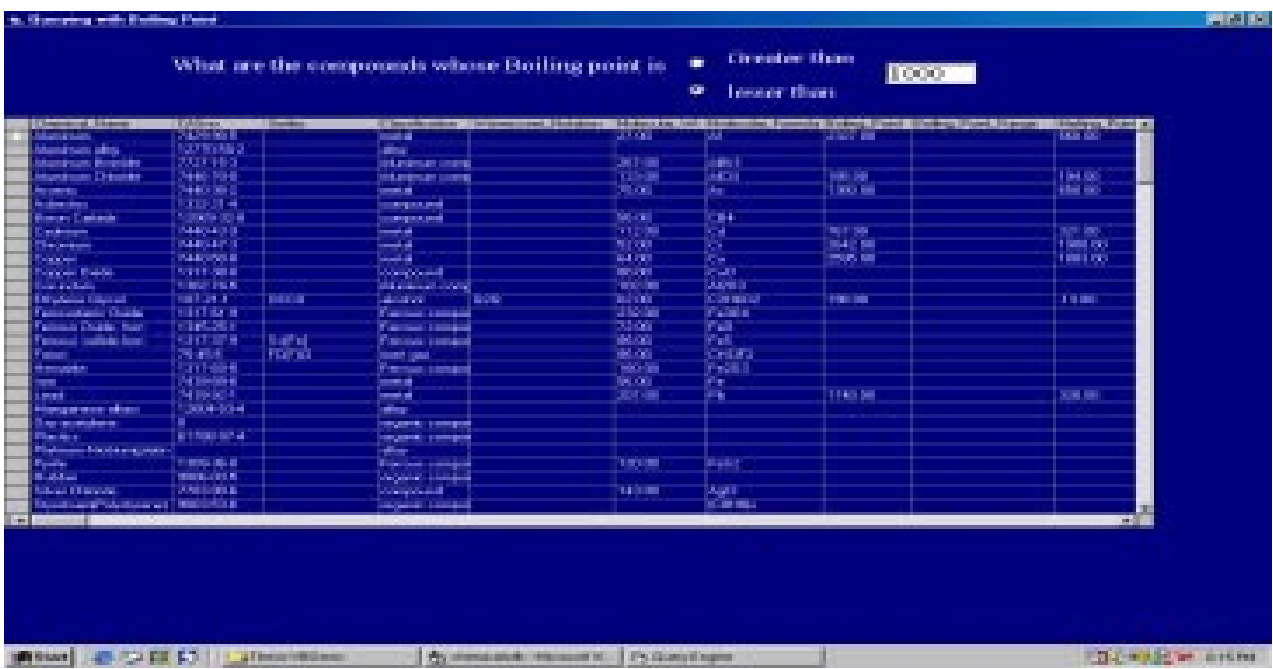

Figure 5.35: Boiling Point Criteria 
The results are as shown below Figure 5.36

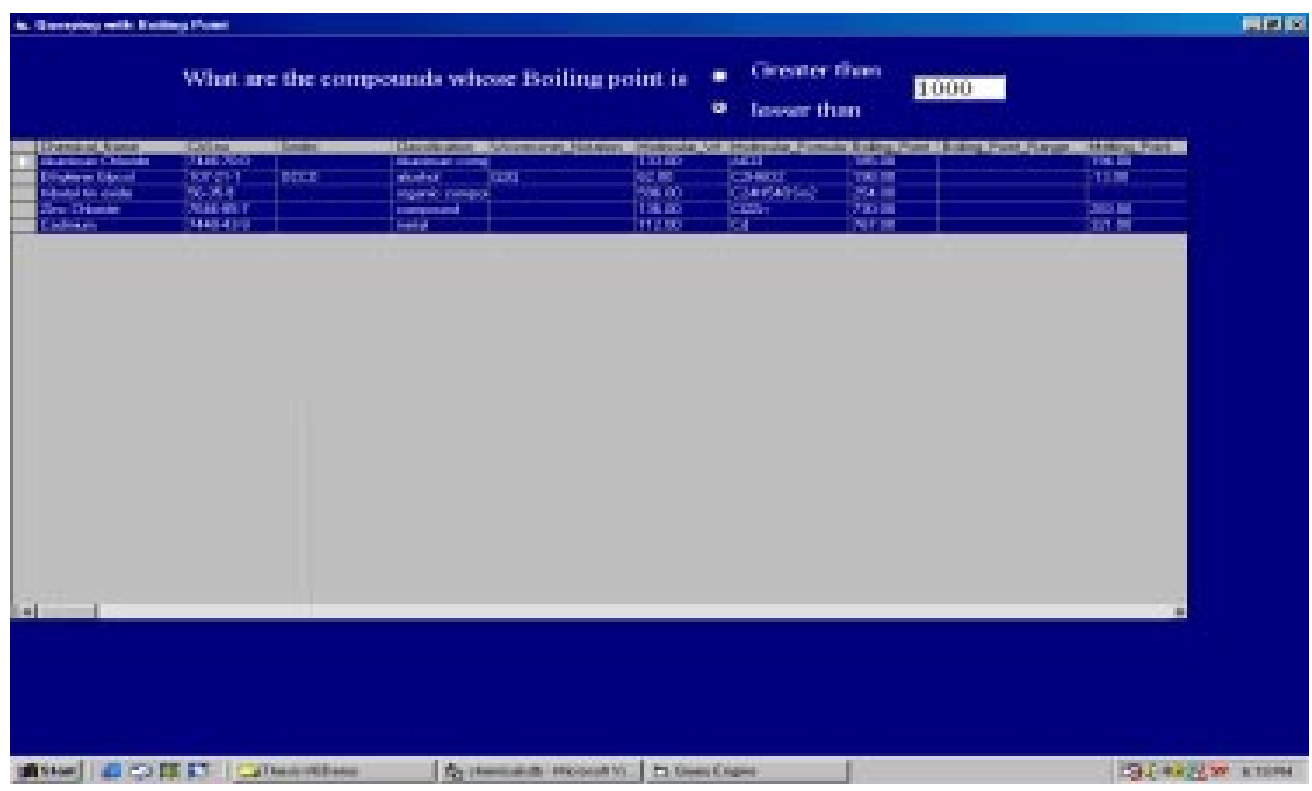

Figure 5.36: Query Results

Querying with Melting Point looks like the Figure 5.37 shown below. This is useful when user wants chemicals and their properties with melting point greater or less than certain value.

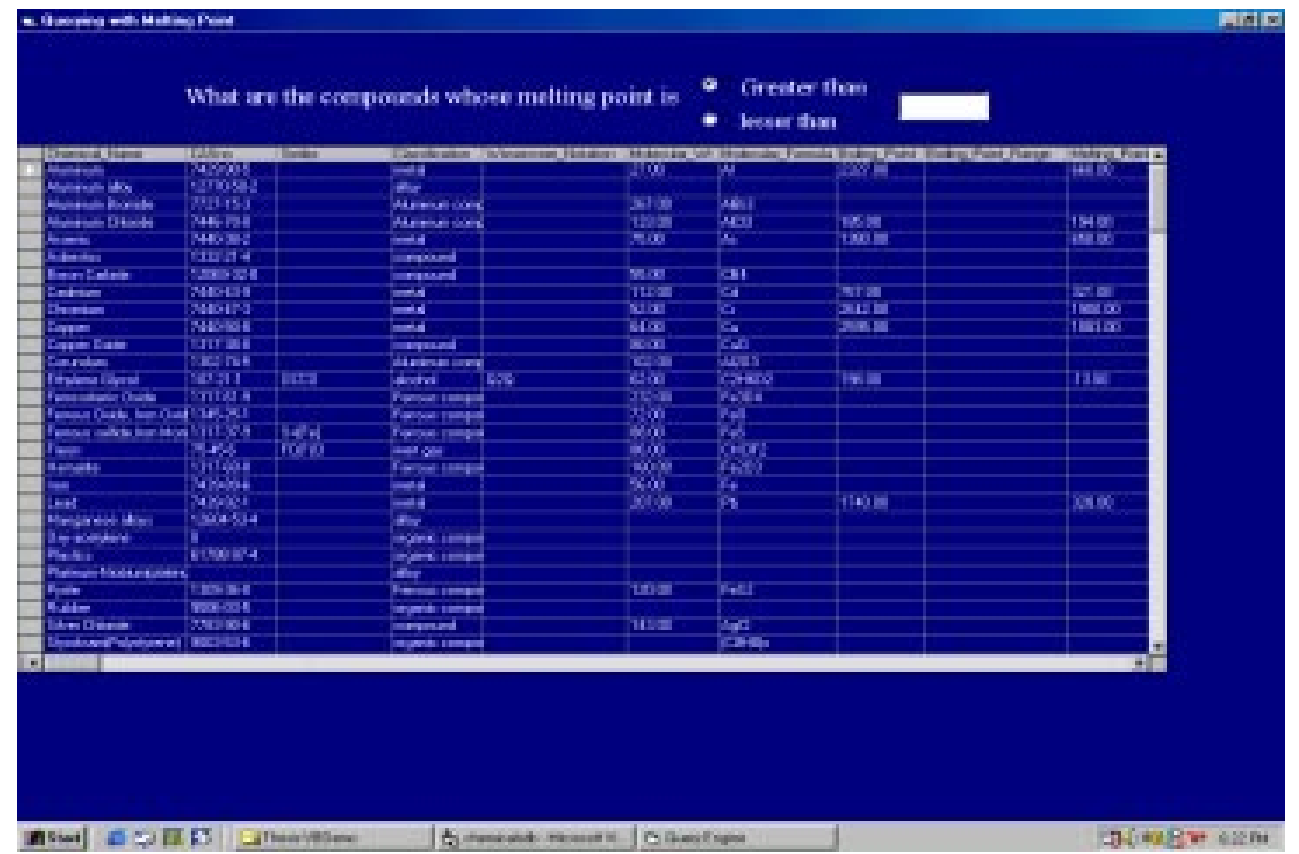

Figure 5.37: Melting Point Query 
Let's say the user enters a value of 500 and he selects greater than, the following results are displayed as shown in Figure 5.38 below

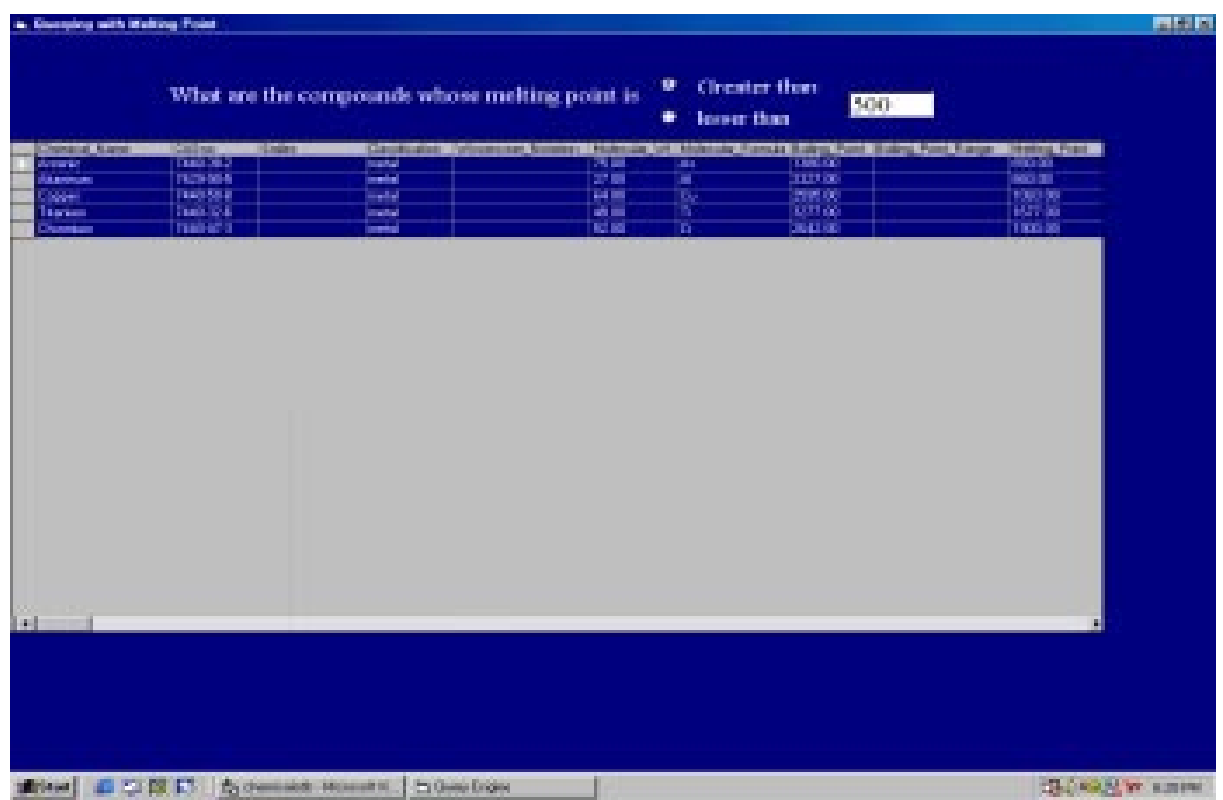

Figure 5.38: Results of Query

The user has an option of finding out what all compounds belonging to a certain family by the following query Figure 5.39

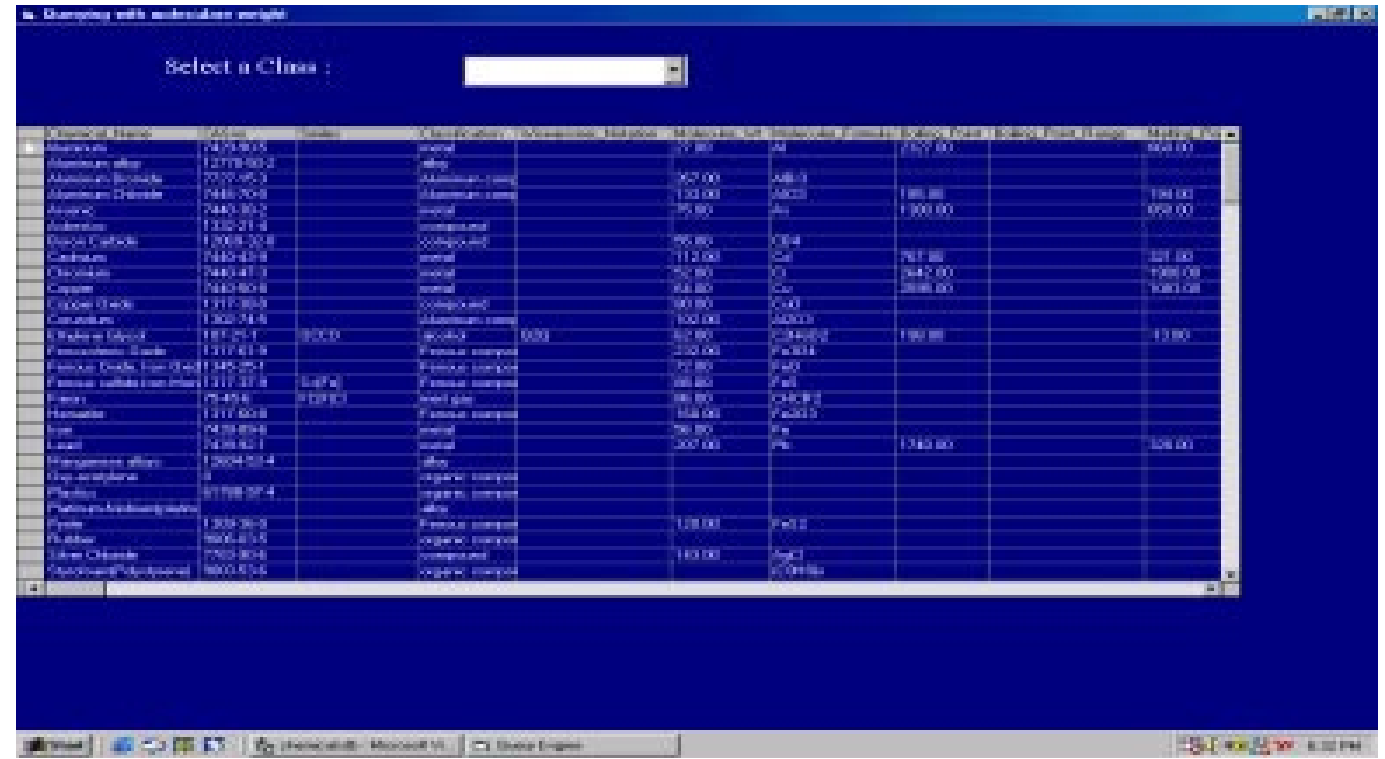

Figure 5.39: Select Any Class Form 
Figure 5.40 below shows the drop down list of various classes of chemicals present

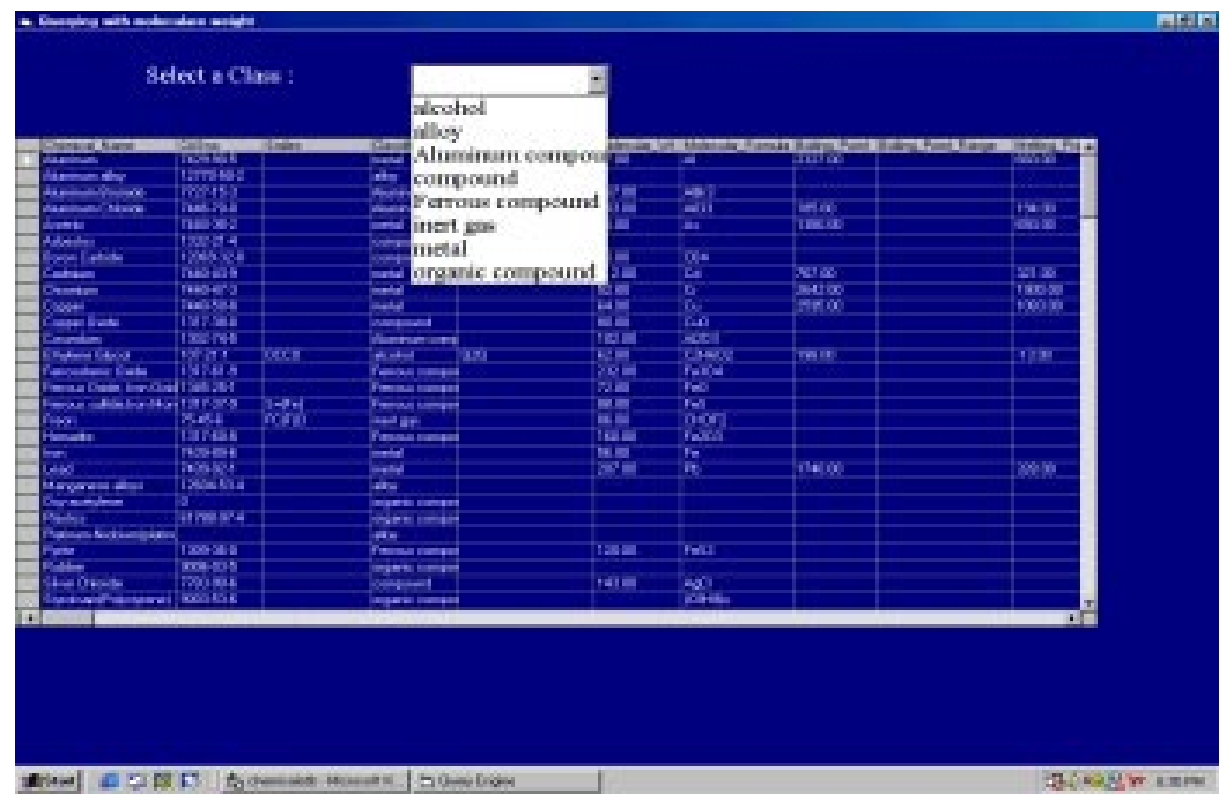

Figure 5.40: Drop down list of various classes of compounds

Lets say the user selected "organic compound", then the following results are displayed Figure 5.41

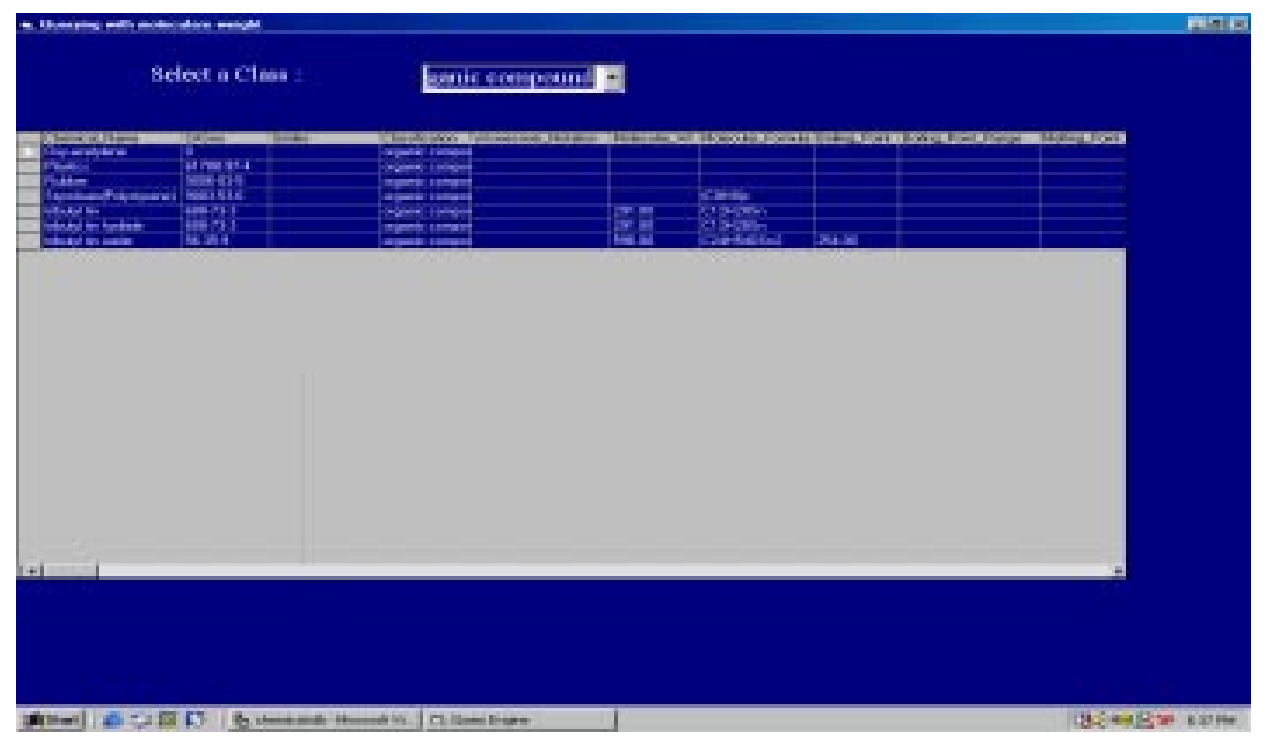

Figure 5.41: Results of Query 
Querying with Vapor pressure form looks like the Figure 5.42 shown below. This is useful when he wants chemicals and their properties with Vapor pressure greater or less than certain value.

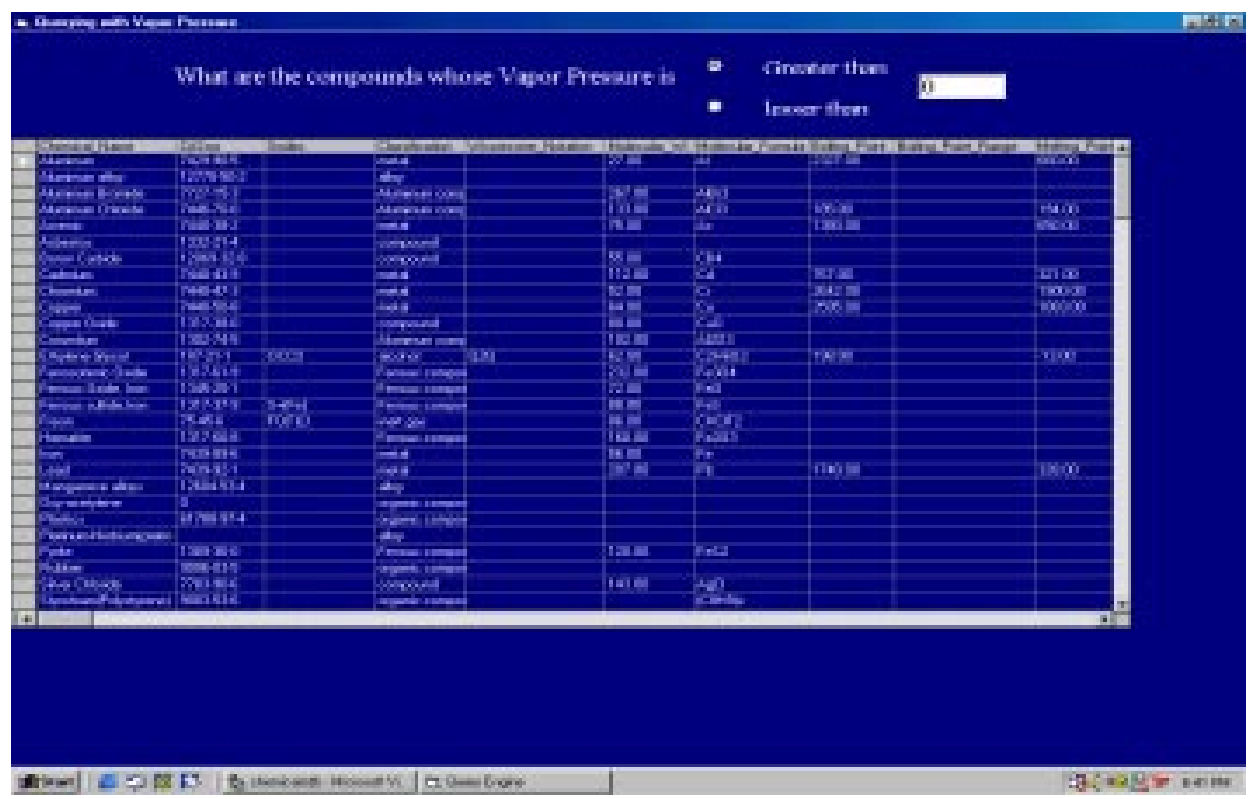

Figure 5.42: Vapor Pressure Query

For example if 0 is entered and greater than is chosen as the option then the following results are displayed Figure 5.43

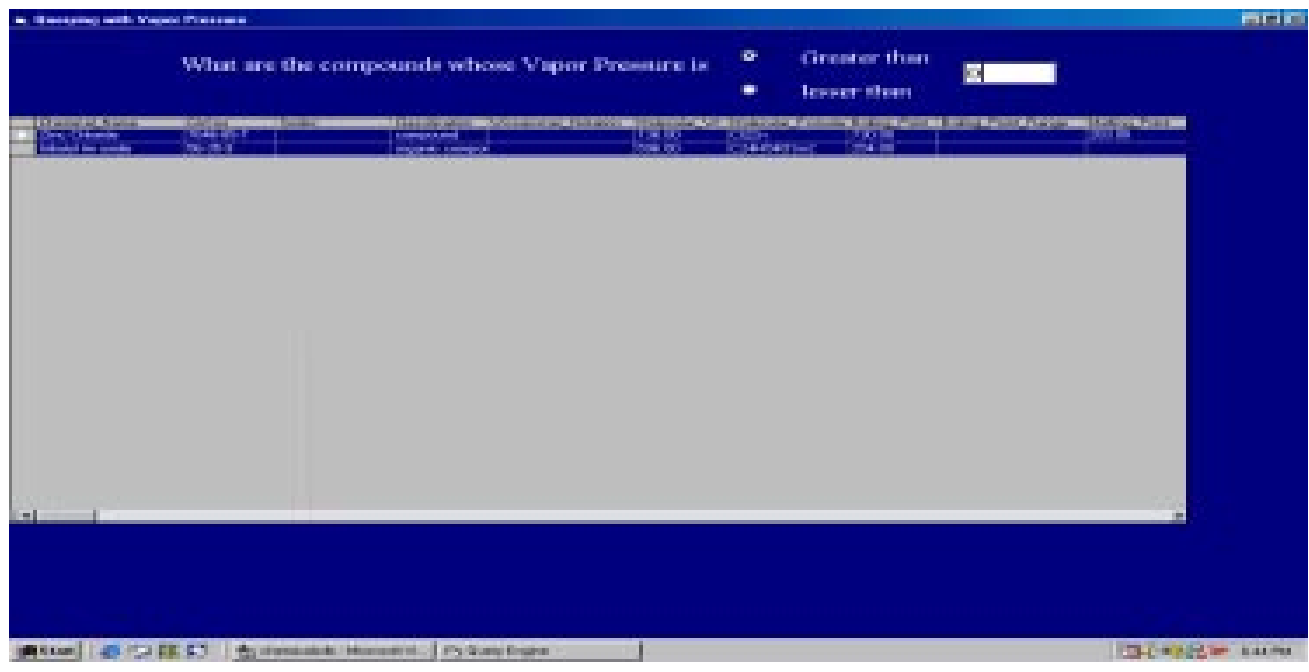

Figure 5.43: Results of Query 
When user clicks on the "help" of the main menu the following screen pops up which helps the user to understand the front end well. Helps him in accessing data using front end

This can be seen from the form below Figure 5.44

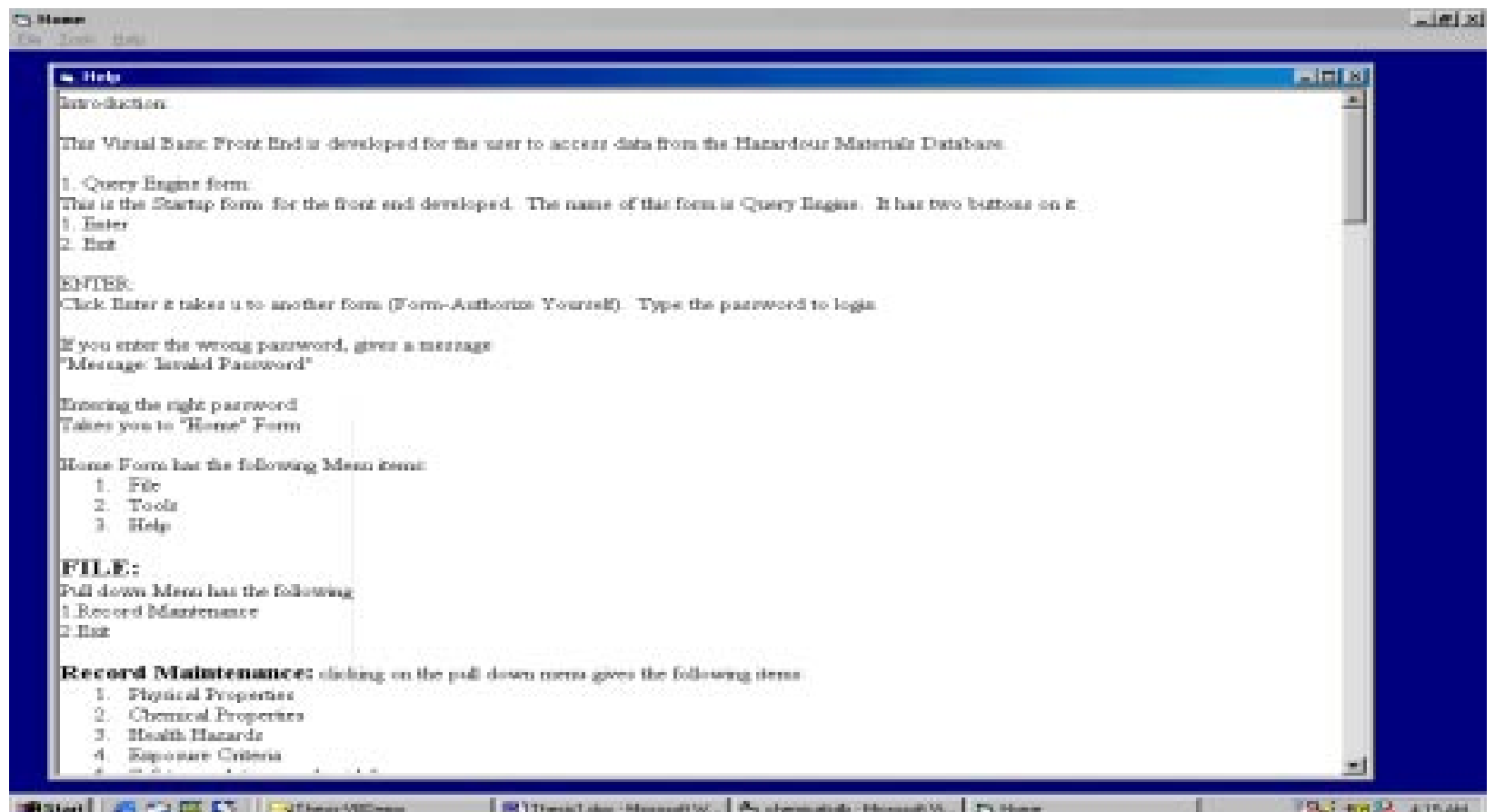

Figure 5.44: Help 


\section{CHAPTER 6: CONCLUSIONS AND FUTURE WORK}

\subsection{Conclusions}

A Hazardous Materials Database (HMD) was developed. HMD stores physical and chemical properties of the chemicals along with their CAS numbers. It also stores data on health hazards, safety regulations and guidelines set by agencies like ACGIH, EPA, and OSHA on exposure criteria and environmental concern. Since this database has all the properties pooled at a single place, it saves look up time. The HMD is made available to a user through a front end developed in Visual Basic. The Graphical User Interface of Visual Basic is easy to use.

The HMD presently has a total of 34 hazardous chemicals with all the above listed properties. The names of the chemicals include aluminum, aluminum alloy, aluminum bromide, aluminum chloride, arsenic, asbestos, boron carbide, cadmium, chromium, copper, copper oxide, corundum, ethylene glycol, ferrosoferric oxide, ferrous oxide, ferrous sulfide, freon, hematite, iron, lead, manganese alloy, oxy acetylene, plastic, platinum-niobium alloy, pyrite, rubber, silver chloride, styrofoam, titanium, titanium alloy, tri butyl tin hydride, tri butyl tin oxide, tri butyl tin, zinc chloride. The Hazardous Materials Database is presently being applied to ship dismantling. By knowing various properties of the chemical the necessary safety measures can be implemented which help in safe handling and disposal of the product, without any detrimental impact on the environment.

\subsection{Future work}

The database can be expanded to Non Hazardous Materials. Apart from the disposal application the HMD database can also be used for recycling of a retired object. This database could be web based so that, it will be easy to access for anyplace and anytime.

The front end of the database is designed only for physical properties. In future the front-end design can be expanded to chemical properties, health hazards, safety regulations and guidelines exposure criteria and environmental concern. 
The database can be used for the entire Life Cycle Design of a Product, from the beginning stages, that is, collection of raw materials to disposal of the product, including recycling.

This is illustrated below.

\section{Expansion of Database to be used over the Life Cycle of a Product:}

Database is designed in such a way that it can be useful over the entire life cycle of a product. To demonstrate this we have added four more tables to the database as shown below, for the example of a ship scrapping.

The different tables added are as follows:

- Main Table

- Ships Table

- Dismantling sites table and

- Cutting technology table

Main Table which has the following fields

- Chemical Name

- Cutting technology

- Ship Type

- Country

Ships table has the following fields:

- Name

- Ship type

- Flag state

- Dead Weight (DWT)

- Build Date

- Retrofit

- Estimated Volume of Hazardous Materials

Dismantling sites Table has the following fields

- Country 
- City

- Capacity

- Safety Record

- Hazardous Material Handling Capability

- Health Hazards

- Labor Cost

Cutting Technology Table has the following fields

- Cutting Technology

- Cutting tool

- Energy Requirement

- Energy Cost

This Database can be used to get many kinds of information regarding a ship, like its location, name of the ship, type of the ship, the different cutting technologies used etc. The whole history of the ship, how it is constructed, what are the hazardous materials that are released during disposal, safety regulations to be followed, health hazards from chemicals released and much more. 


\section{References}

1. J. Grant, 1987-Logical Introduction to Databases Towson State University -Harcourt Brace Jovanovich Publishers

2. S. L. Alter, 1980-DECISION SUPPORT SYSTEMS-Current practices and continuing Challenges.

3. R. H. Sprague, Jr and H. J. Watson, 1986-DECISION SUPPORT SYSTEMS-Putting theory into Practice.

4. US Bureau of Mines - Automobile Disposal- A National Problem-Case studies of factors that influence the accumulation of Automobile Scrap.

5. http://www2.ccc.uni-erlangen.de/services/webmol.html- The WWW Chemical Structures Database.

6. http://www.tus.com.au/default.htm - The Chemical Inventory Database

7. http://dippr.byu.edu/samplesite/chemsearch.asp - Sample chemical Database

8. http://math.cd-rom-di.../482.Brethericks.Reactive.Chemical.Hazards.Databas.shtmBretherick's Reactive Chemical Hazards Database Version 2.0

9. http://www.wiz.uni-kassel.de/dain/ddb/x284.html- Hazardous Chemical Database

10. http://www.genome.ad.jp/manuscripts/GIW95/Poster/GIW95P27.html- LIGAND Chemical Database for Enzymatic Reactions: a link between enzyme structures and chemical reactions.

11. http://www.unitednuclear.com/chemical.html- United Nuclear Chemical Database

12. http://ull.chemistry.uakron.edu/erd - Hazardous chemical Database

13. P. Patnaik, 1999 - A Comprehensive guide to the Hazardous Properties of Chemical substances- second edition, John Wiley \& Sons, Inc., Publication.

14. P. H. Howard, 1990 -Handbook of Environmental Fate and Exposure Data For Organic Chemicals- Lewis Publishers, volume II.

15. P. H. Howard and M. Neal, - Dictionary of Chemical Names and Synonyms

16. R. P. Pohanish and S. A. Greene, 1996- Hazardous Materials Handbook.

17. J. C. Prager, Volume I, 1995-Environmental Contaminant Reference Data book.

18. “Guide For Handling Hazardous Materials”- UPS March 1973 
19. J. H. MEIDL, 1972- "Hazardous Materials Handbook"-Glencoe press.

20. U.S Department of Transportation, 1978- "Emergency Action Guide for Selected Hazardous Materials"

21. Schieler and Pauze, 1976 - "Hazardous Materials"- Van Nostrand Reinhold Company

22. M. Sittig, 1976 - "Toxic Metals Pollution Control and Worker Protection"- Noyes Data Corporation

23. P. N. Cheremisinoff, 1987, Pudvan Publishing Co - "A Guide to Working with Hazardous Materials" 


\section{APPENDIX 1 - ACUTE SYMPTOMS TABLE}

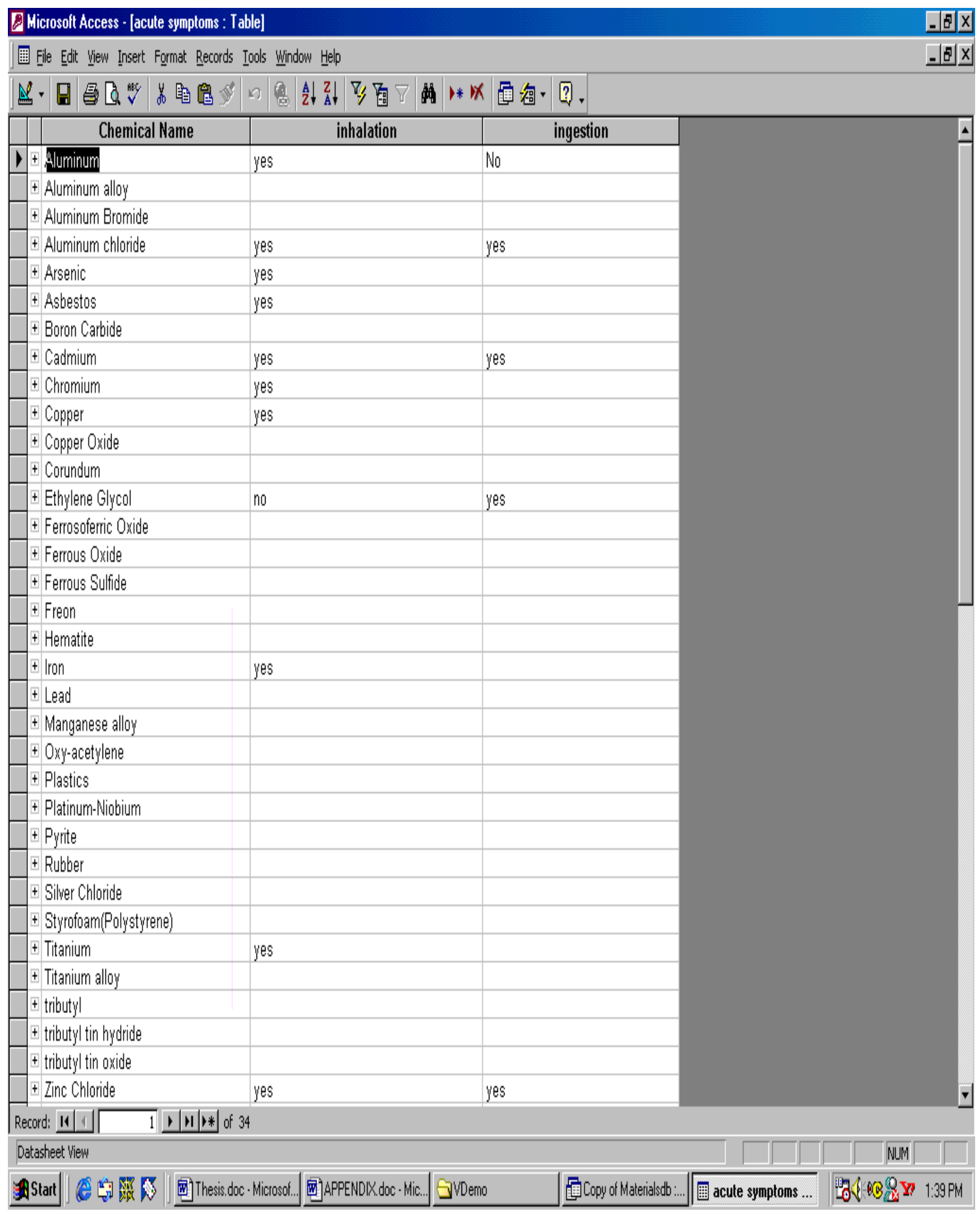




\section{APPENDIX 2 - ALLERGEN TABLE}

\section{Q Microsolt Access - [allergen : Table]}

国 File Edit Wiew Insert Format Records Iools Window Help

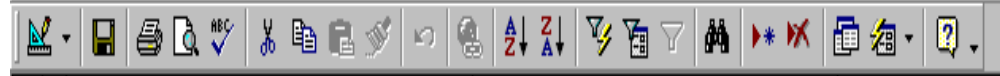

\begin{tabular}{|c|c|c|c|c|c|c|c|}
\hline \multicolumn{2}{|c|}{ Chemical Name } & skin & & respiratory & \multicolumn{3}{|c|}{ other } \\
\hline I Aluminum & & & yes & & Brain & & \\
\hline \multicolumn{8}{|l|}{ + Aluminum alloy } \\
\hline \multicolumn{8}{|l|}{ † Aluminum Bromide } \\
\hline \multicolumn{8}{|l|}{ + Aluminum Chloride } \\
\hline \pm Arsenic & & yes & \multicolumn{2}{|l|}{ yes } & \multicolumn{3}{|l|}{ Liver } \\
\hline \pm Asbestos & & & yes & & \\
\hline \multicolumn{8}{|l|}{ + Bron Carbide } \\
\hline \pm Cadmium & & & yes & & \multicolumn{3}{|l|}{ Liver } \\
\hline † Chromium & & yes & yes & & \multicolumn{3}{|l|}{ Kidneys } \\
\hline \pm Copper & & yes & yes & & & & \\
\hline \multicolumn{8}{|l|}{ \pm Copper Oxide } \\
\hline \multicolumn{8}{|l|}{$\mp$ Corundum } \\
\hline \pm Ethylene Glycol & & yes & no & & \multicolumn{3}{|l|}{ Eyes } \\
\hline \multicolumn{8}{|l|}{ + Ferrosoferric Oxide } \\
\hline \multicolumn{8}{|c|}{ + Ferrous Oxide, Iron Oxide } \\
\hline \multicolumn{8}{|c|}{ † Ferrous sulfide, lron Monosulfide } \\
\hline \multicolumn{8}{|l|}{ \pm Freon } \\
\hline \multicolumn{8}{|l|}{ \pm Hematite } \\
\hline$\mp$ Iron & & & yes & & & & \\
\hline \multicolumn{8}{|l|}{ \pm Lead } \\
\hline \multicolumn{8}{|l|}{ \pm Manganese alloy } \\
\hline \multicolumn{8}{|l|}{ $\pm 0 x y$-acetylene } \\
\hline \pm Plastics & & & \multicolumn{2}{|l|}{ yes } & \multicolumn{3}{|l|}{ eyes, nose } \\
\hline \multicolumn{8}{|l|}{ † Platinum-Niobium } \\
\hline \multicolumn{8}{|l|}{ \pm Pyrite } \\
\hline \pm Rubber & & & & & & & \\
\hline + Silver Chloride & & & & & & & \\
\hline † Styrofoam(Polystyren & & & & & & & \\
\hline \pm Titanium & & & yes & & & & \\
\hline † Titanium alloy & & & & & & & \\
\hline \pm tributyl tin & & & & & & & \\
\hline t tributyl tin hydride & & & & & & & \\
\hline I tributyl tin oxide & & & & & & & \\
\hline \pm Zinc Chloride & & & & & & & $\nabla$ \\
\hline Record: $11 \mid 1 \longdiv { \square }$ & $1|1| 1 \mid * 4$ of 34 & & & & & & \\
\hline Datasheet Yiew & & & & & & NUM & \\
\hline Start $\Leftrightarrow$ & 国 Thesis.doc-Microsof... & GVDemo & 馬Copy of Mateilasodb:. & 国)APPENDIX.doc - Mic.... & 国 allergen : Table & 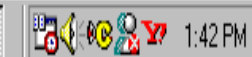 & \\
\hline
\end{tabular}




\section{APPENDIX 3 - AQUATIC FATE TABLE}

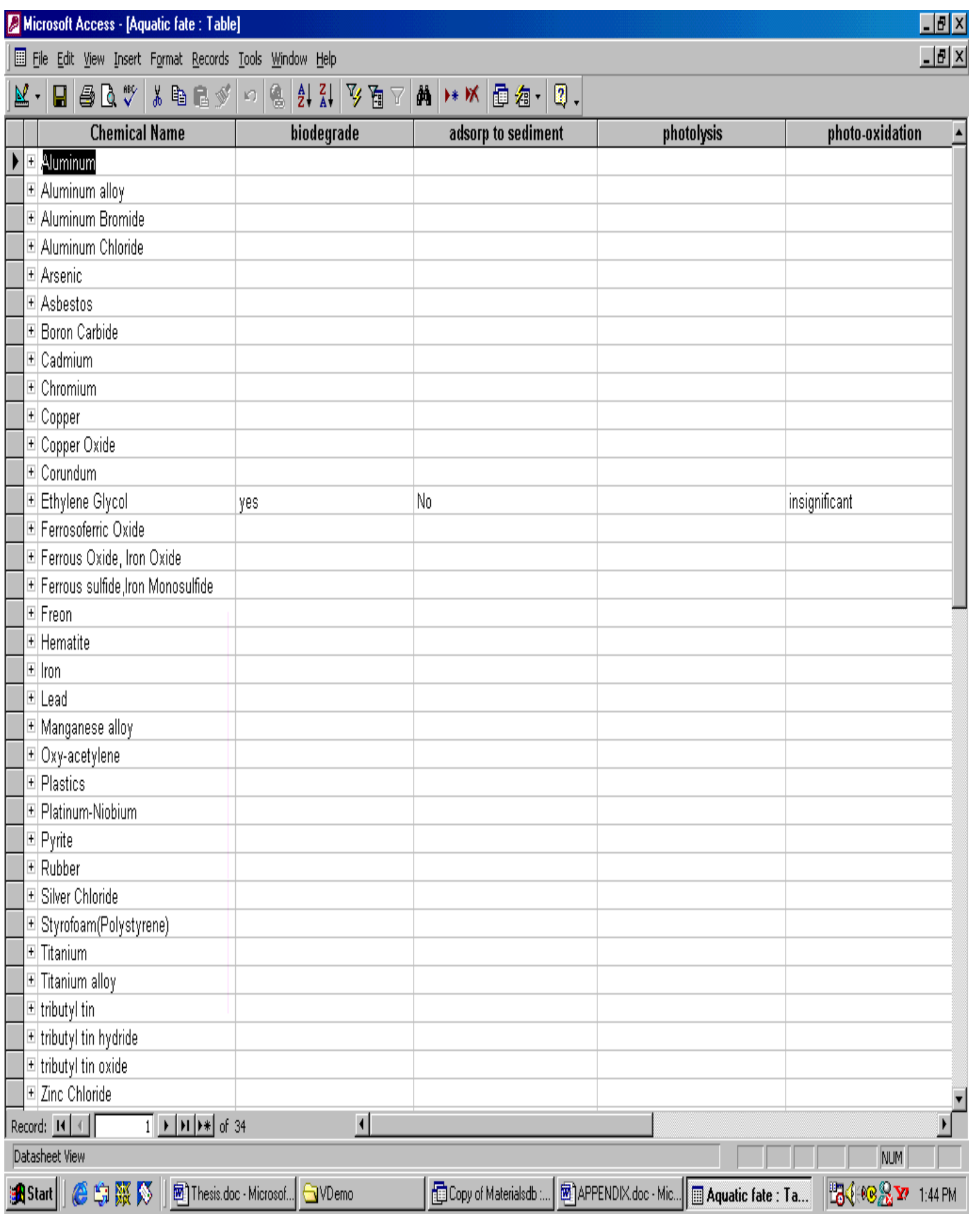




\section{APPENDIX 4 - ATMOSPHERIC FATE TABLE}

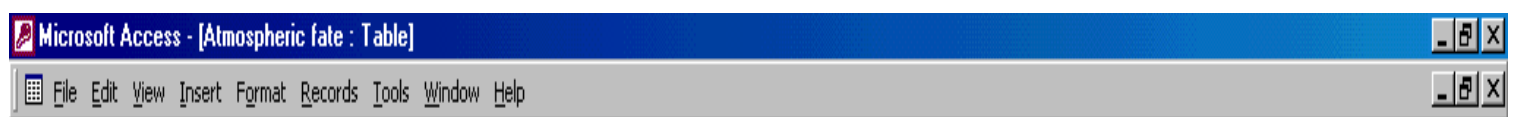

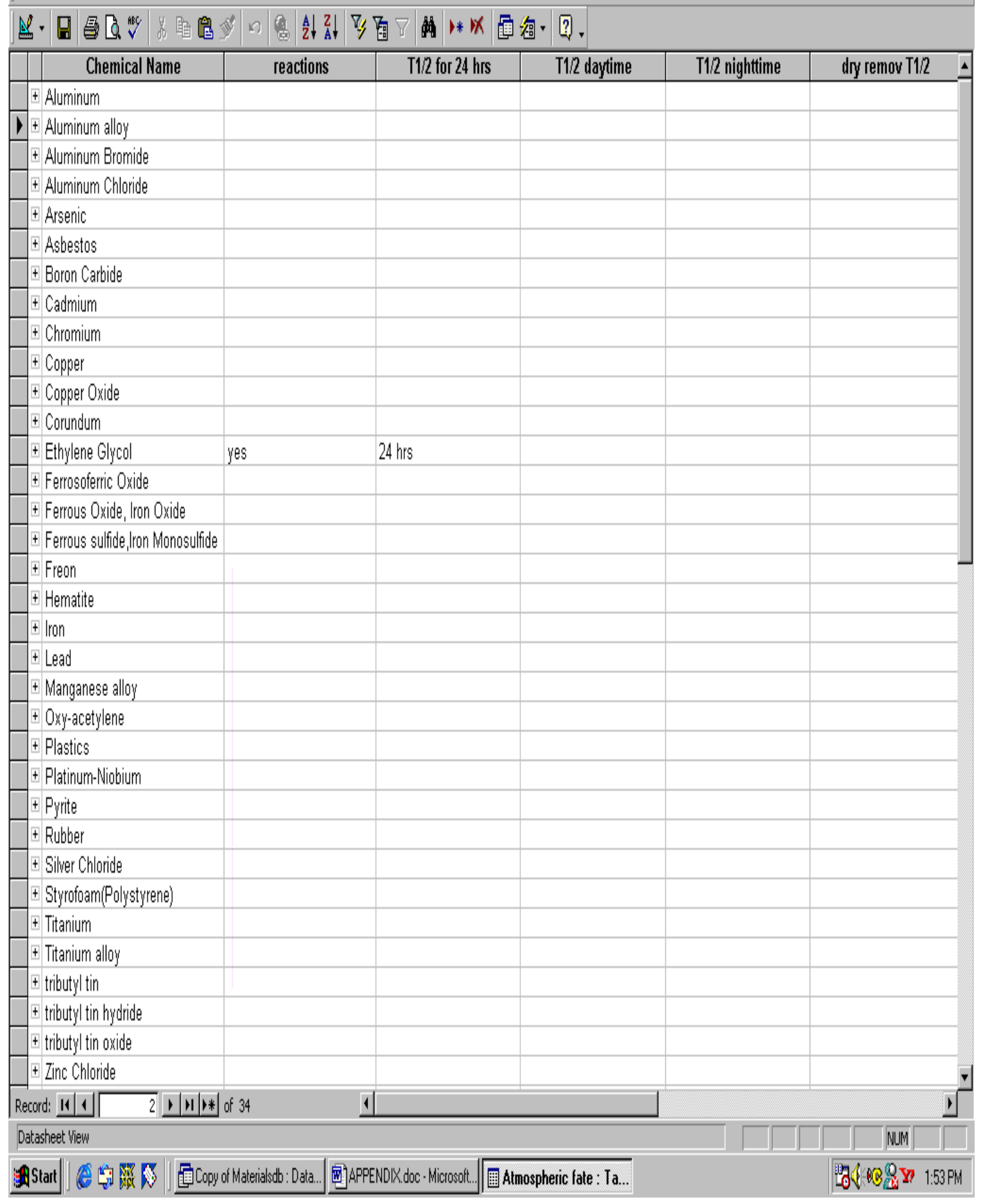




\section{APPENDIX 5 - BOD TABLE}

Q Microsolt Access - [BOD : Table] $\quad$ -

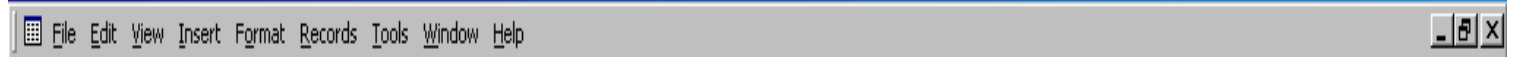

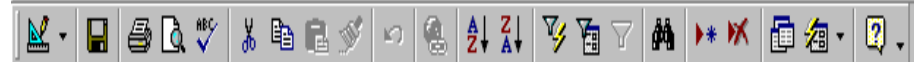

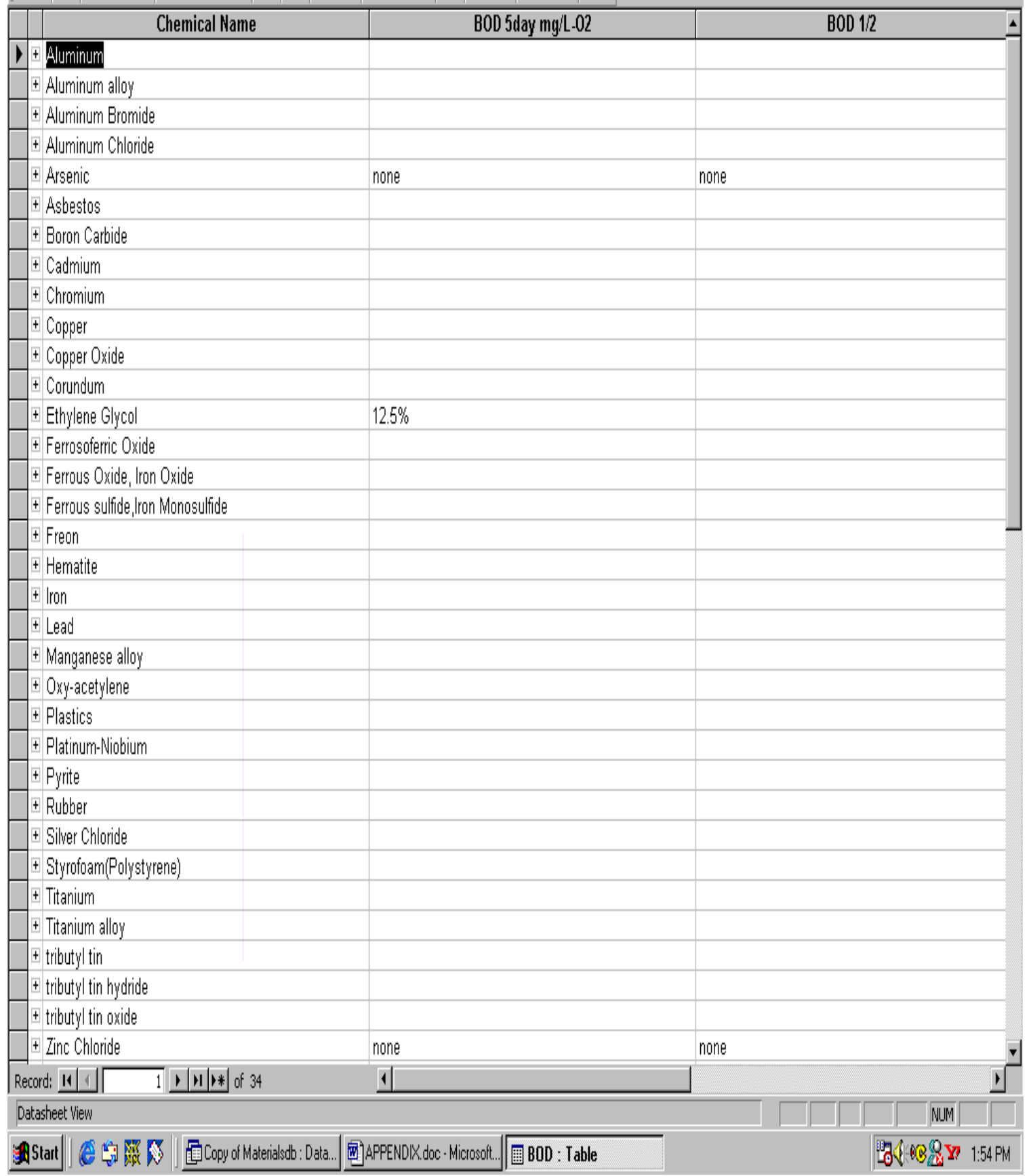




\section{APPENDIX 6 - CANCER TABLE}

Q Microsolt Access - [cancer : Table]

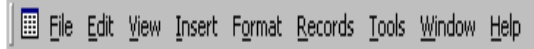

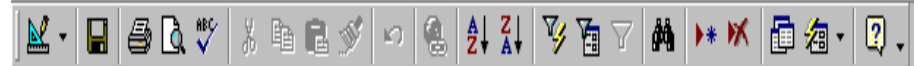

\begin{tabular}{|c|c|c|c|c|c|c|}
\hline Chemical Nan & mutagenic & genotoxic & promoter & Lung & others & Gastrointestinal $\Delta$ \\
\hline † Aluminum & No & No & No & & & \\
\hline \multicolumn{7}{|l|}{ † Aluminum alloy } \\
\hline \multicolumn{7}{|l|}{ \pm Aluminum Bromide } \\
\hline \multicolumn{7}{|l|}{ † Aluminum Chloride } \\
\hline$\mp$ Arsenic & yes & & & yes & kidneys, liver & yes \\
\hline \pm Asbestos & & & & & & yes \\
\hline \multicolumn{7}{|l|}{ \pm Boron Carbide } \\
\hline † Cadmium & & yes & & & & \\
\hline \pm Chromium & & & & yes & & \\
\hline \multicolumn{7}{|l|}{ † Copper } \\
\hline \multicolumn{7}{|l|}{ \pm Copper Oxide } \\
\hline \multicolumn{7}{|l|}{ \pm Corundum } \\
\hline \multicolumn{7}{|l|}{ \pm Ethylene Glycol } \\
\hline \multicolumn{7}{|l|}{ + Ferrosoferric Oxide } \\
\hline \multicolumn{7}{|c|}{ + Ferrous Oxide, lron Oxide } \\
\hline \multicolumn{7}{|c|}{ † Ferrous sulfide, Iron Monosulfide } \\
\hline \multicolumn{7}{|l|}{ t Freon } \\
\hline \multicolumn{7}{|l|}{$\mp$ Hematite } \\
\hline \multicolumn{7}{|l|}{ I lron } \\
\hline \multicolumn{7}{|l|}{ \pm Lead } \\
\hline \multicolumn{7}{|l|}{ † Manganese alloy } \\
\hline \multicolumn{7}{|l|}{ \pm Oxy-acetylene } \\
\hline \multicolumn{7}{|l|}{ † Plastics } \\
\hline \multicolumn{7}{|l|}{ \pm Platinum-Niobium } \\
\hline \multicolumn{7}{|l|}{ † Pyrite } \\
\hline \multicolumn{7}{|l|}{ I Rubber } \\
\hline \multicolumn{7}{|l|}{ + Silver Chloride } \\
\hline \multicolumn{7}{|l|}{ \pm Styrofoam(Polystyrene) } \\
\hline \multicolumn{7}{|l|}{ \pm Titanium } \\
\hline \multicolumn{7}{|l|}{ + Titanium alloy } \\
\hline \multicolumn{7}{|l|}{ \pm tributyl tin } \\
\hline \multicolumn{7}{|l|}{ \pm tributyl tin hydride } \\
\hline I tributyl tin oxide & & & & & & \\
\hline \pm Zinc Chloride & & & & & & 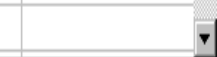 \\
\hline Record: $1 1 1 1 \longdiv { 5 }$ & $1,111 * *$ of 34 & & & & & \\
\hline Dotasheet Yiew & & & & & & NUM \\
\hline Start 04 & 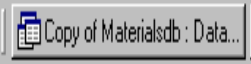 & 国_LPPENDIX_doc- Micrososolt... & $\sqrt{\text { : cancer : Table }}$ & & & 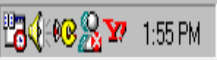 \\
\hline
\end{tabular}




\section{APPENDIX 7 - RCRA TABLE}

Q Microsolt Access - [characterisicic RCRA : Table]

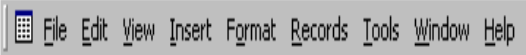

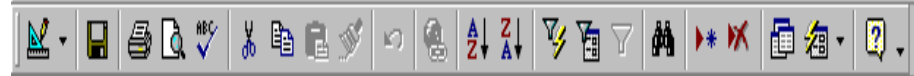

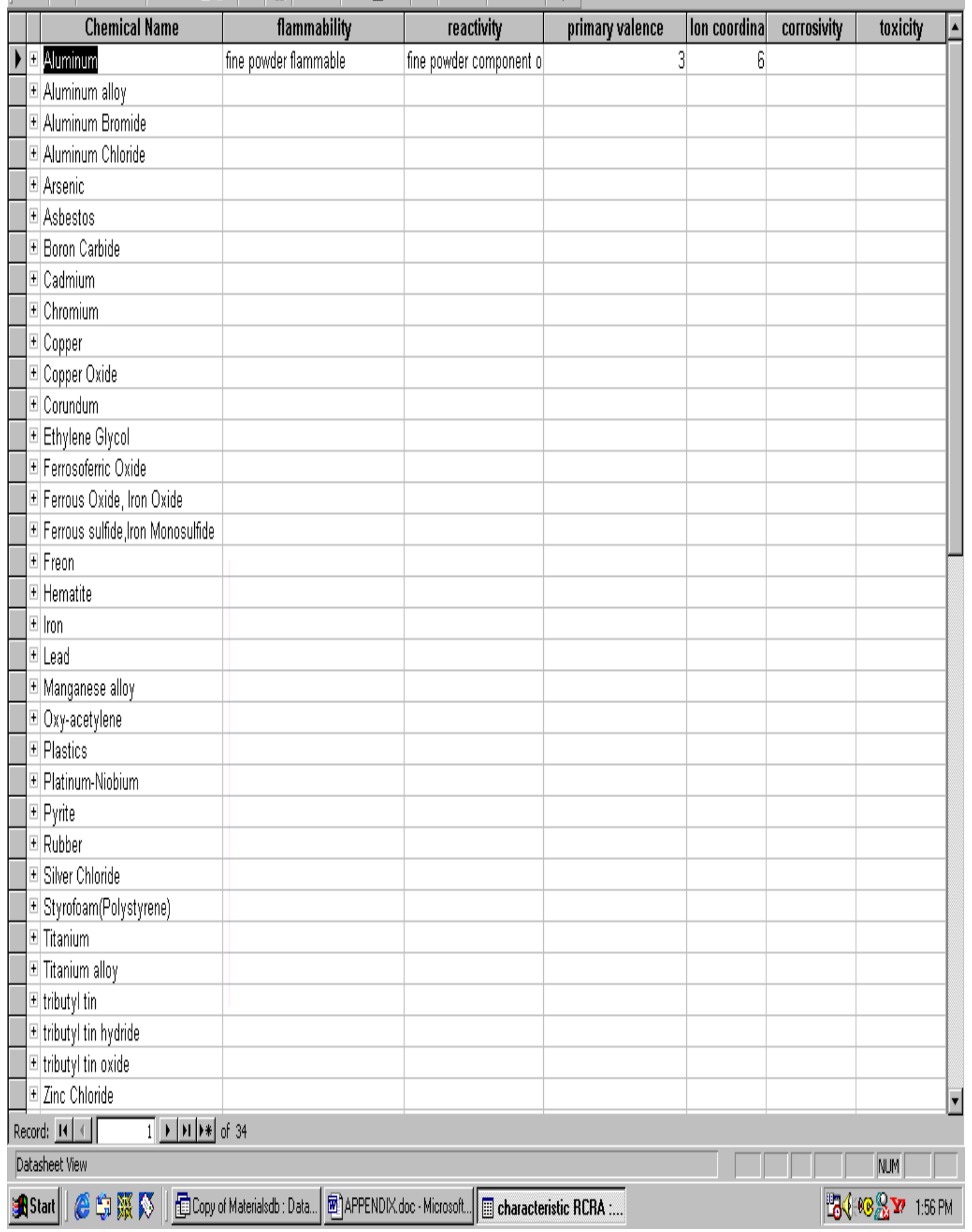




\section{APPENDIX 8 - CHRONIC SYMPTOMS TABLE}

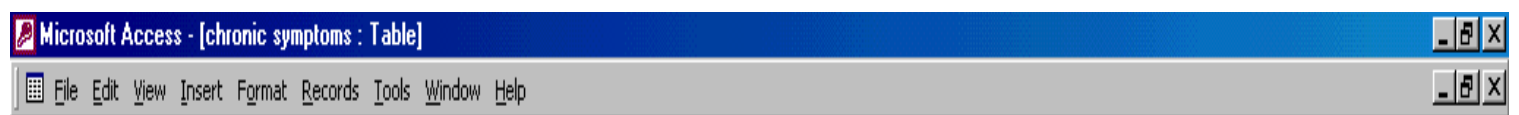

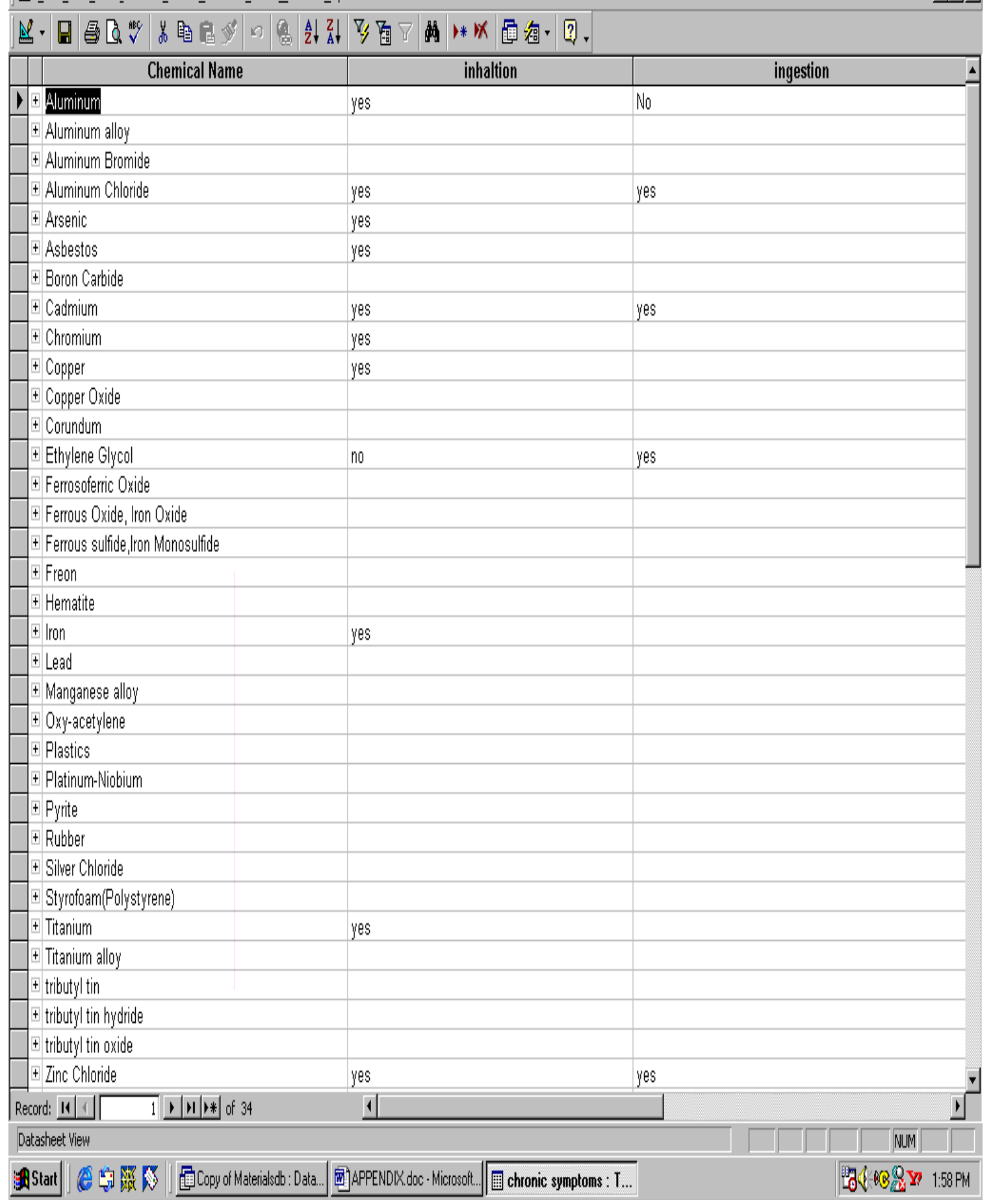




\section{APPENDIX 9 - CLEAN AIR ACT TABLE}

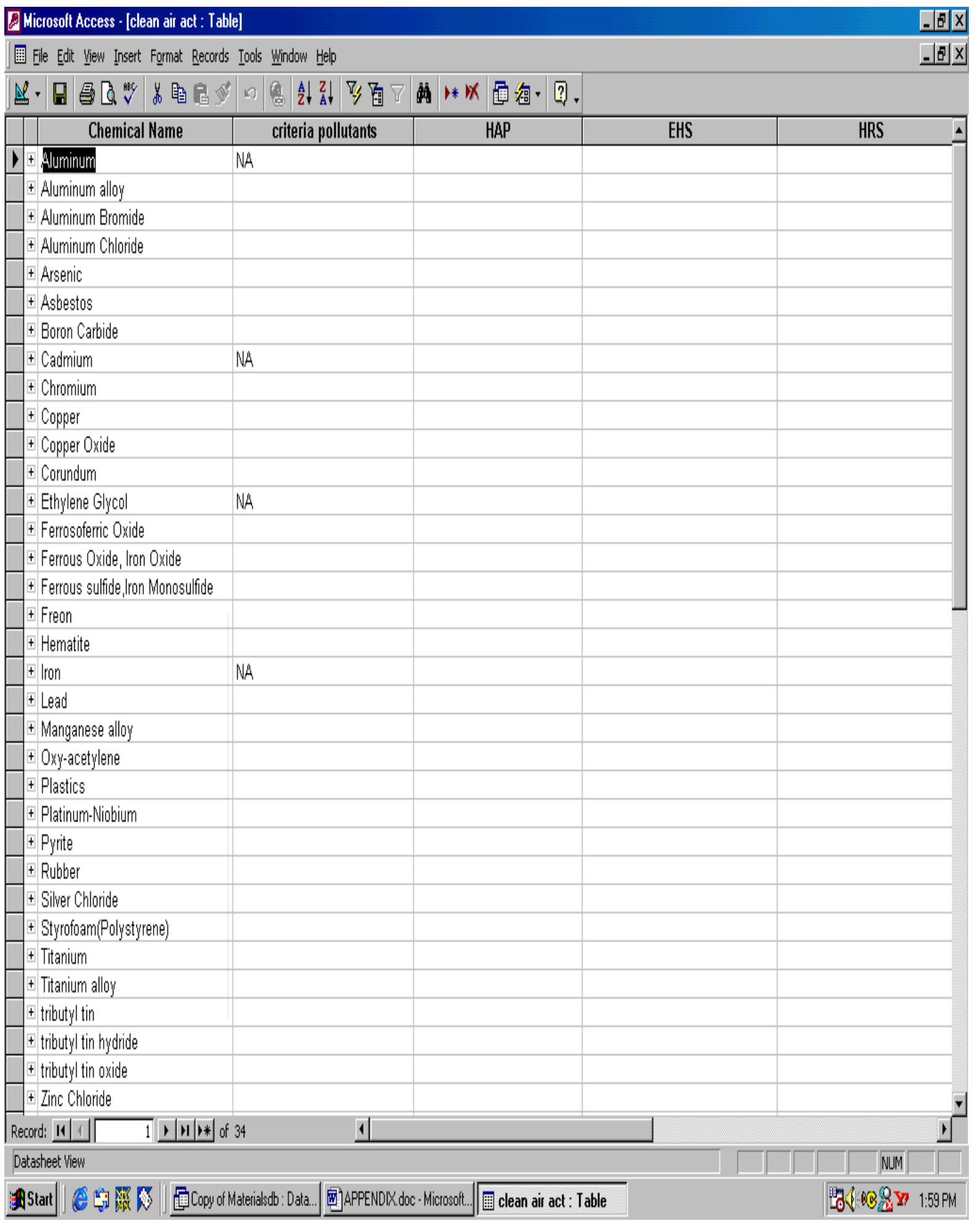




\section{APPENDIX 10 - CORROSIVITY TABLE}

\section{[Ricrosoft Access - [corrosivity: Table]}

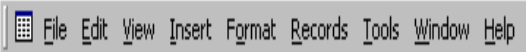

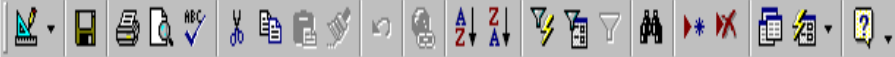

\begin{tabular}{|c|c|c|c|c|c|c|}
\hline Chemica & | Name & toward Steel & toward Flesh & \multicolumn{2}{|c|}{ Materials which corrode material } & $\Delta$ \\
\hline I Aluminum & & & & \multicolumn{2}{|c|}{ Acids, other metals } & \\
\hline † Aluminum alloy & & & & \multicolumn{2}{|l|}{ 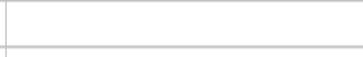 } & \\
\hline + Aluminum Bromide & & & & & \\
\hline † Aluminum Chloride & no & & no & & \\
\hline$\mp$ Arsenic & & & & & \\
\hline \pm Asbestos & & & & & \\
\hline \pm Boron Carbide & & & & & \\
\hline \pm Cadmium & & & & & \\
\hline † Chromium & & & & & \\
\hline † Copper & & & & & \\
\hline \pm Copper Oxide & & & & & \\
\hline$\mp$ Corundum & & & & & \\
\hline ¿ Ethylene Glycol & & & & & \\
\hline t Ferrosoferric Oxide & & & & & \\
\hline + Ferrous Oxide, Iron 0 & & & & & \\
\hline + Ferrous sulfide, fron $M$ & lonosulfide & & & & \\
\hline t Freon & & & & & \\
\hline + Hematite & & & & & \\
\hline Etron & & & & & \\
\hline \pm Lead & & & & & \\
\hline + Manganese alloy & & & & & \\
\hline $\pm 0 x y$-acetylene & & & & & \\
\hline + Plastics & & & & & \\
\hline \pm Platinum-Niobium & & & & & \\
\hline + Pyrite & & & & & \\
\hline † Rubber & & & & & \\
\hline \pm Silver Chloride & & & & & & \\
\hline + Styrofoam(Polystyren & & & & & & \\
\hline + Titanium & & & & & & \\
\hline \pm Titanium alloy & & & & & & \\
\hline \pm tributyl tin & & & & & & \\
\hline \pm tributyl tin hydride & & & & & & \\
\hline † tributyl tin oxide & & & & & & \\
\hline \pm Zinc Chloride & & & & & & $\nabla$ \\
\hline Record: $11 \mid 1 \longdiv { 1 }$ & $1.1110 *$ of 34 & & & & & \\
\hline Datasheet Yiew & & & & & NMM & . \\
\hline Start 640 & 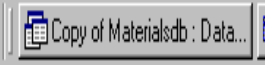 & 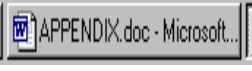 & 娄 corrosivity : Table & & DLOMD & \\
\hline
\end{tabular}




\section{APPENDIX 11 - DOT TABLE}

[QMicrosolt Access - [DOT : Table]

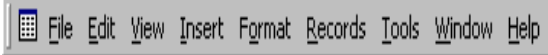

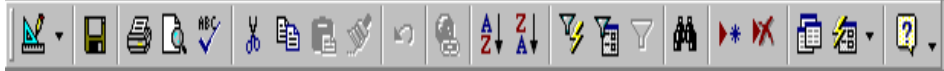

\begin{tabular}{|c|c|c|c|}
\hline Chemical Name & water pollution & hazard class & registration pesticide FIFRA \\
\hline \pm Aluminum & & & \\
\hline \pm Aluminum alloy & & & \\
\hline † Aluminum Bromide & & & \\
\hline † Aluminum Chloride & harmful to acquatic life & 8 & \\
\hline \pm Arsenic & dangerous if it enters water & 6.1 & \\
\hline + Asbestos & & & \\
\hline † Boron Carbide & & & \\
\hline + Cadmium & & & \\
\hline \pm Chromium & & & \\
\hline \pm Copper & & & \\
\hline+ Copper Oxide & & & \\
\hline \pm Corundum & & & \\
\hline \pm Ethylene Glycol & dangerous if enters water,acquatic life $\cdot 9$ & 9 & \\
\hline \pm Ferrosoferric Oxide & & & \\
\hline \pm Ferrous Oxide, Iron Oxide & & & \\
\hline \pm Ferrous sulfide, Kron Monosulfide & & & \\
\hline \pm Freon & & & \\
\hline \pm Hematite & & & \\
\hline \pm lron & & & \\
\hline$\mp$ Lead & & & \\
\hline \pm Manganese alloy & & & \\
\hline $\pm 0 x y$-acetylene & & & \\
\hline \pm Plastics & & & \\
\hline \pm Platinum-Niobium & & & \\
\hline \pm Pyrite & & & \\
\hline \pm Rubber & & & \\
\hline \pm Silver Chloride & & & \\
\hline \pm Styrofoam(Polystyrene) & & & \\
\hline \pm Titanium & & & \\
\hline \pm Titanium alloy & & & \\
\hline \pm tributyl tin & & & \\
\hline \pm tributyl tin hydride & & & \\
\hline \pm tributyl tin oxide & & & \\
\hline \pm Zinc Chloride & harmful to aquatic life, dangerous when 8 & & \\
\hline
\end{tabular}

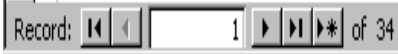

Dotasheet View

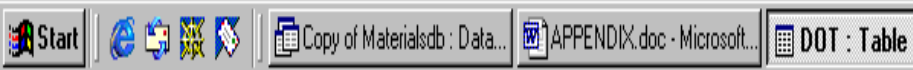




\section{APPENDIX 12 - EXTINGUISHING AGENTS TABLE}

\section{Q Microsolt Access - [extinguishing agents : Table]}

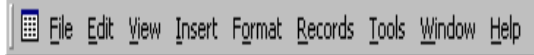

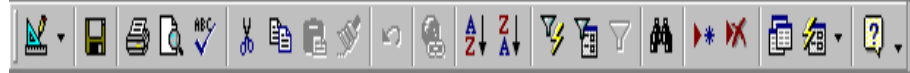

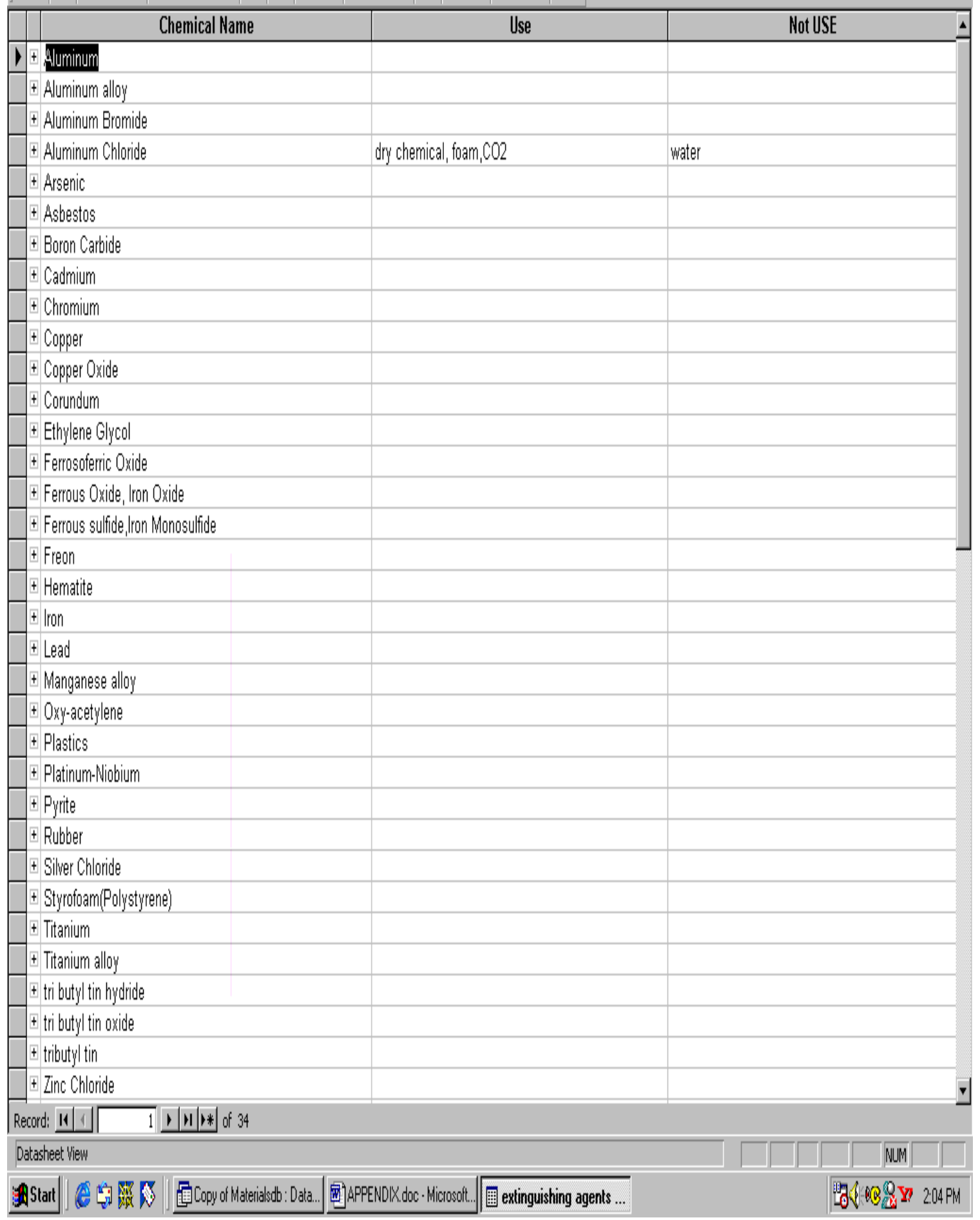




\section{APPENDIX 13 - FLAMMABILITY/COMBUSTIBILITY TABLE}

Q Microsolt Access - [Flammability/combustability : Table]

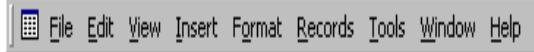

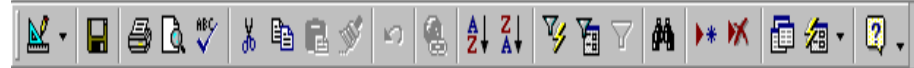

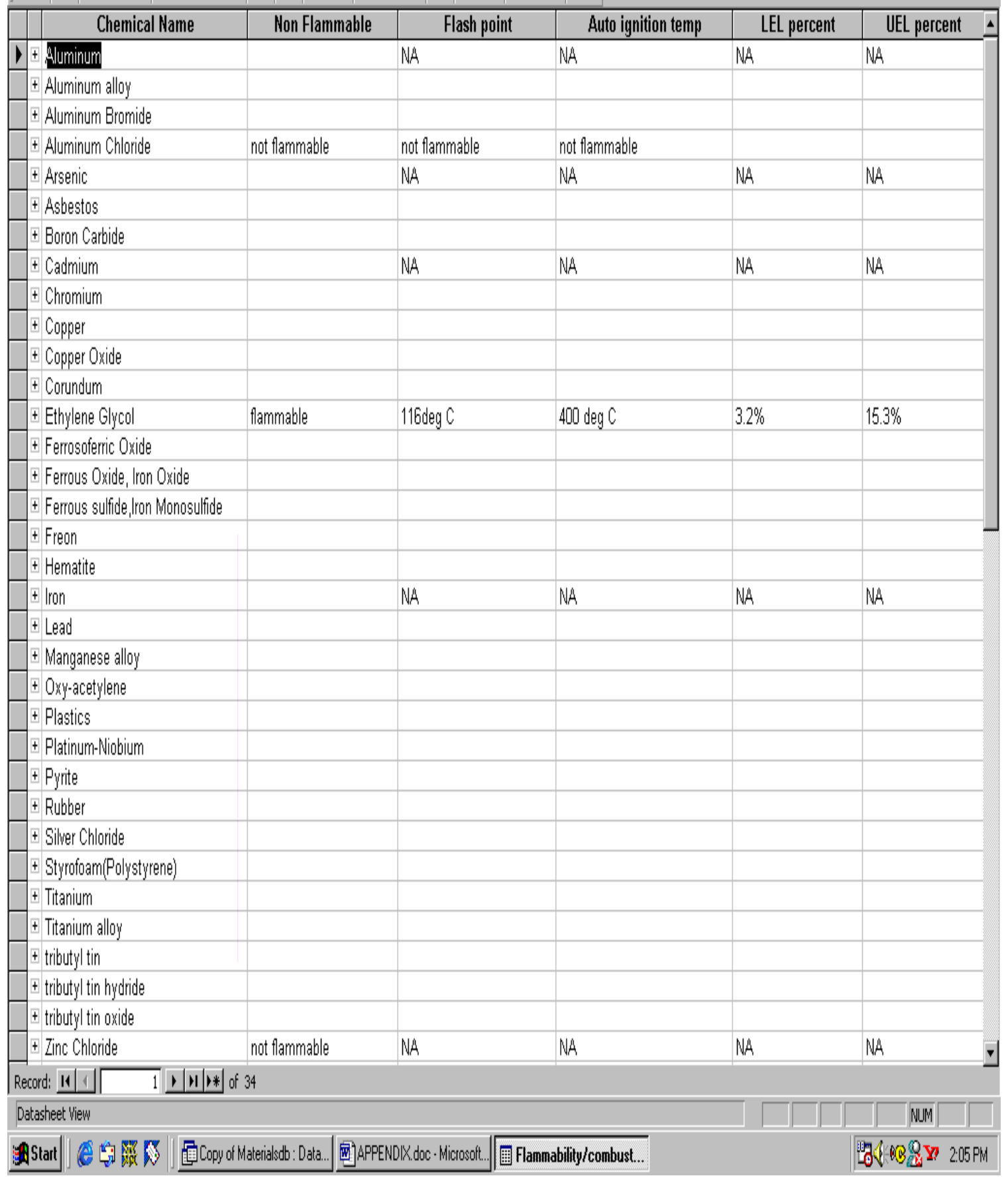




\section{APPENDIX 14 - IRRITANT TABLE}

\section{Q Microsolt Access - [iritant: Table]}

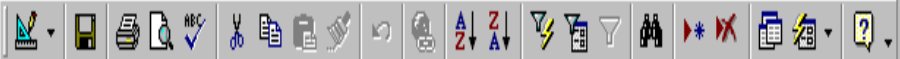

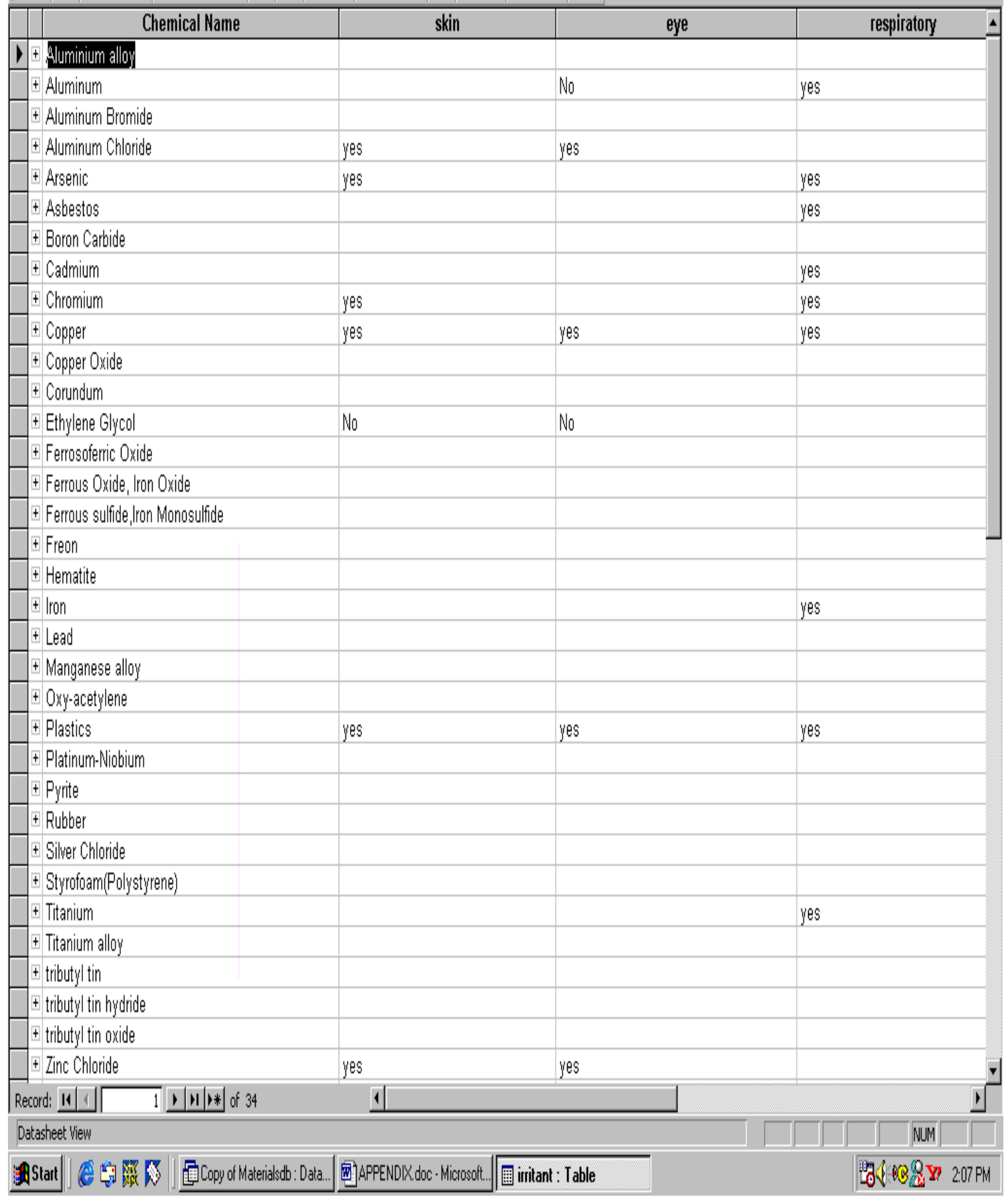




\section{APPENDIX 15 - MATERIALS TABLE}

Q Microsoft Access - [Materials : Table]

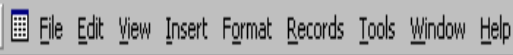

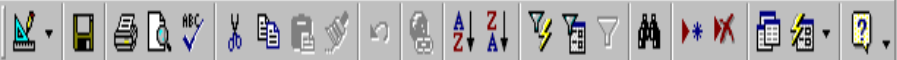

\begin{tabular}{|c|c|c|c|c|c|c|c|c|c|c|c|c|c|}
\hline Chemical_Nar & an CASno & Smiles & \begin{tabular}{|l|} 
Classification \\
\end{tabular} & Wis | & Molecu Molecu B & Boiling | & Melting_Point Log & Ii Densi| & VP H & \begin{tabular}{|l|l|l|} 
Henry's lea & 0 r \\
\end{tabular} & & Sources & $\Delta$ \\
\hline I + Aluminum & 7429.90 .5 & & metal & & $27 \mathrm{Al}$ & 2327 & 660 & 2.698 & & & \multicolumn{3}{|c|}{ P. H. Howard an Volat } \\
\hline \pm Aluminum alloy & 12770.50.-2 & & alloy & & & & & & & & \multicolumn{3}{|c|}{ P. H. Howard an Volat } \\
\hline † Aluminum Brom & orm $7727 \cdot 15 \cdot 3$ & & Aluminum comp & & $267 A B B B$ & & & & & & \multicolumn{3}{|c|}{ P. H. Howard an } \\
\hline ‡ Aluminum Chlor & lot $7446.70-0$ & & Aluminum comp & & $133 \mathrm{AICB}$ & 185 & 194 & 2.44 & & & \multicolumn{3}{|c|}{ R. P. Pohanish a Volat } \\
\hline \pm Arsenic & $7440-30 \cdot 2$ & & metal & & $75 \mathrm{As}$ & 1380 & 650 & & & & \multicolumn{3}{|c|}{ R. P. Pohanish a Volat } \\
\hline$\mp$ Asbestos & 1332-21-4 & & compound & & & & & & & & \multicolumn{3}{|c|}{ P. H. Howard an Volat } \\
\hline \pm Boron Carbide & 120699.32 .8 & & compound & & 55 CB4 & & & & & & \multicolumn{3}{|c|}{ P. H. Howard an Volat } \\
\hline + Cadmium & $7440-43.9$ & & metal & & $112 \mathrm{Cd}$ & 767 & 321 & & & & \multicolumn{3}{|c|}{$500 \mathrm{~m}$ P. H. Howard an Volat } \\
\hline I Chromium & $7440-47 \cdot 3$ & & metal & & $52 \mathrm{Cr}$ & 2642 & 1900 & 7.14 & & & \multicolumn{3}{|c|}{ P. H. Howard an Volat } \\
\hline \pm Copper & $7440-50-8$ & & metal & & $64 \mathrm{Cu}$ & 2595 & 1083 & 8.94 & & & \multicolumn{3}{|c|}{ P. H. Howard an Volat } \\
\hline \pm Copper Oxide & $1317-3800$ & & compound & & $80 \mathrm{CUO}$ & & & & & & \multicolumn{3}{|c|}{ P. H. Howard an Volat } \\
\hline \pm Corundum & $1302.74 \cdot 5$ & & Aluminum comp & & $102 \mathrm{~A} 1203$ & & & & & & \multicolumn{3}{|c|}{ P. H. Howard an } \\
\hline † Ethylene Glycol & 101 107-21-1 & OCCO & alcohol & 026 & $62 \mathrm{C}^{2} \mathrm{HOO}:$ & 198 & $.13 \cdot 1.34$ & & & $6 \times 10 E-8$ yes $5 t$ & \multicolumn{3}{|c|}{ to 1P. H. Howard an Volat } \\
\hline † Ferrosofoeric 0 \% & $0 \times 1317-61 \cdot 9$ & & Ferrous compol & & $232 \mathrm{Fe} 304$ & & & & & & \multicolumn{3}{|c|}{ P. H. Howard an } \\
\hline † Ferrous Oxide, & $1345-25 \cdot 1$ & & Ferrous compol & & $72 \mathrm{FeO}$ & & & & & & \multicolumn{3}{|c|}{ P. H. Howard an } \\
\hline ¿ Ferrous sulfide, & e. 1317.37 .9 & $S=[F e]$ & Ferrous compol & & $88 \mathrm{FeS}$ & & & & & & \multicolumn{3}{|c|}{ P. H. Howard an } \\
\hline t Freon & $75-45-6$ & FC(F)Cl & inert gas & & 86 CHCF2 & & & & & & \multicolumn{3}{|c|}{ P. H. Howard an Volar } \\
\hline I Hematite & $1317-60]-8$ & & Ferrous compol. & & $160 \mathrm{Fe} 203$ & & & & & & \multicolumn{3}{|c|}{ P. H. Howard an } \\
\hline I lron & 7439.89 .6 & & metal & & $56 \mathrm{Fe}$ & & & & & & \multicolumn{3}{|c|}{ P. H. Howard an Volal } \\
\hline \pm Lead & $7439.92 \cdot 1$ & & metal & & $207 \mathrm{~Pb}$ & 1740 & 328 & 11.35 & & & \multicolumn{3}{|c|}{ P. H. Howard an Volat } \\
\hline † Manganese allo & $1012604-53-4$ & & alloy & & & & & & & & \multicolumn{3}{|c|}{ P. H. Howard an Volal } \\
\hline \pm Oxy-acetylene & & & organic compou & & & & & & & & \multicolumn{3}{|c|}{ P. H. Howard an Volat } \\
\hline + Plastics & $61788-97-4$ & & organic compou & & & & & & & & \multicolumn{3}{|c|}{ P. H. Howard an Volal } \\
\hline † Platinum-Niobiu & & & alloy & & & & & & & & & H. Howard an & Volat \\
\hline † Pyite & $1309-36-0$ & & Ferrous compol & & $120 \mathrm{FeS} 2$ & & & & & & & H. Howard an & \\
\hline \pm Rubber & 90060.03 .5 & & organic compou & & & & & & & & & H. Howard an & Volal \\
\hline † Silver Chloride & $7783.90-6$ & & compound & & $143 \mathrm{AgCl}$ & & & & & & & H. Howard an & Volal \\
\hline † Styrofoam(Poly: & y: 9003.53 .6 & & organic compou & & (COHB):x & & & & & & & H. Howard an & Volal \\
\hline \pm Titanium & $7440-32 \cdot 6$ & & metal & & $48 \mathrm{Ti}$ & 3277 & 1677 & & & & & H. Howard an & \\
\hline \pm Titanium alloy & $7440-32 \cdot 6$ & & alloy & & $48 \mathrm{Ti}$ & & & & & & & H. Howard an & Volat \\
\hline \pm tributyl tin & $6808-73-3$ & & organic compou & & $291 \mathrm{C} 12 \mathrm{H} 28$ & & & & & & & H. Howard an & \\
\hline I tributyl tin hydrile & ril 6808-73-3 & & organic compou & & $291 \mathrm{C} 12 \mathrm{H} 28$ & & & & & & & H. Howard an & \\
\hline + tributyl tin oxides & e 56.35 .9 & & organic compou & & $596 \mathrm{C2} 24554$ & 254 & & 1.17 & 1 & & $94 m P .1$ & H. Howard an & Volat \\
\hline \pm Zinc Chloride 7 & $7646.85-7$ & & compound & & $136 \mathrm{C} 122 \mathrm{n}$ & 730 & 283 & 2.91 & 1 & & & P. Pohanish a & Volat \\
\hline Record: $11|1|$ & $1,1.1$ & 1* of 34 & & 11 & & & & & & & & & 1 \\
\hline Dotasheet Yiew & & & & & & & & & & & & NUM & \\
\hline Start (c) & 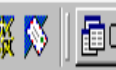 & Copy of Mateiria & 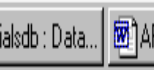 & APPENDI & IX_doc-Microsoft... & 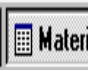 & erials: Table & & & & & SOQBD & :O9PM \\
\hline
\end{tabular}




\section{APPENDIX 16 - ORAL ACUTE AQUATIC TOXICITY TABLE}

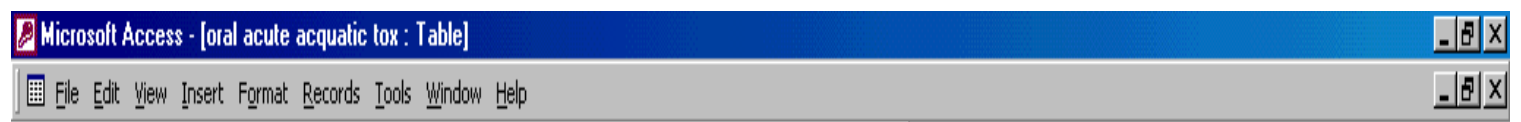

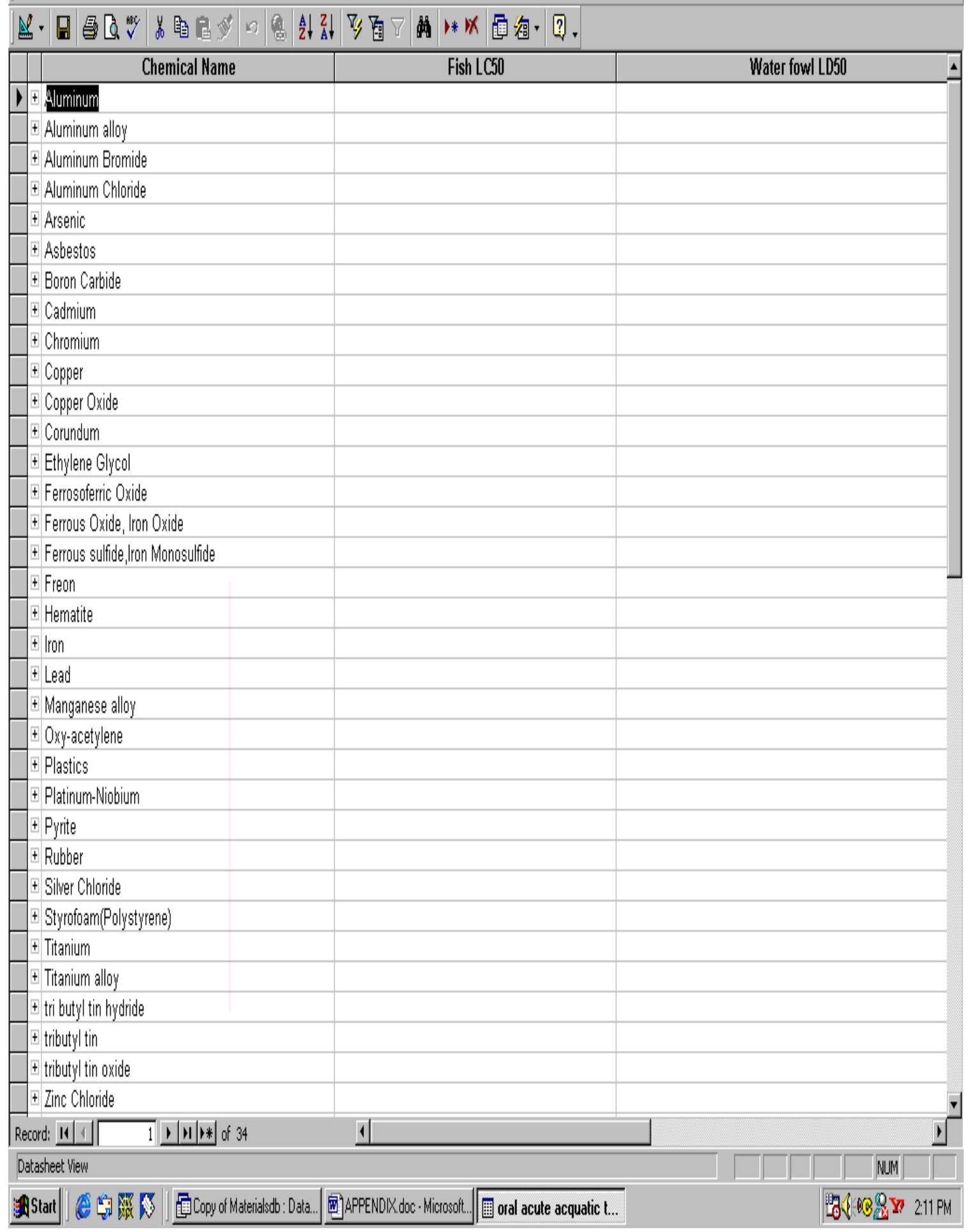




\section{APPENDIX 17 - PERCENT DISSOCIATED TABLE}

\section{Q Microsoft Access - [Percent Dissociated : Table]}

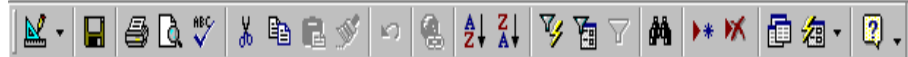

\begin{tabular}{|c|c|c|c|c|c|}
\hline Chemical & Name & $\mathrm{PH} 4$ & $\mathrm{PH7}$ & PH9 & PH11 \\
\hline \multicolumn{6}{|l|}{ 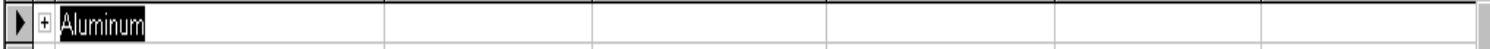 } \\
\hline \multicolumn{6}{|l|}{ + Aluminum alloy } \\
\hline \multicolumn{6}{|l|}{ + Aluminum Bromide } \\
\hline \multicolumn{6}{|l|}{ † Aluminum Chloride } \\
\hline \multicolumn{6}{|l|}{ \pm Arsenic } \\
\hline \multicolumn{6}{|l|}{+ Asbestos } \\
\hline \multicolumn{6}{|l|}{ \pm Boron Carbide } \\
\hline \multicolumn{6}{|l|}{ + Cadmium } \\
\hline \multicolumn{6}{|l|}{ \pm Chromium } \\
\hline \multicolumn{6}{|l|}{ \pm Copper } \\
\hline \multicolumn{6}{|l|}{ \pm Copper Oxide } \\
\hline \multicolumn{6}{|l|}{+ Corundum } \\
\hline \multicolumn{6}{|l|}{ + Ethylene Glycol } \\
\hline \multicolumn{6}{|l|}{ + Ferrosoferric Oxide } \\
\hline \multicolumn{6}{|c|}{ + Ferrous Oxide, Iron Oxide } \\
\hline \multicolumn{6}{|c|}{ † Ferrous sulfide, lron Monosulfide } \\
\hline \multicolumn{6}{|l|}{ \pm Freon } \\
\hline \multicolumn{6}{|l|}{ \pm Hematite } \\
\hline \multicolumn{6}{|l|}{ \pm Iron } \\
\hline \multicolumn{6}{|l|}{+ Lead } \\
\hline \multicolumn{6}{|l|}{ + Manganese alloy } \\
\hline \multicolumn{6}{|l|}{ $\pm 0 x y$-acetylene } \\
\hline \multicolumn{6}{|l|}{ \pm Plastics } \\
\hline \multicolumn{6}{|l|}{ + Platinum-Niobium } \\
\hline \multicolumn{6}{|l|}{ \pm Pyrite } \\
\hline \multicolumn{6}{|l|}{ \pm Rubber } \\
\hline \multicolumn{6}{|l|}{ \pm Silver Chloride } \\
\hline + Styrofoam(Polystyrer & & & & & \\
\hline \pm Titanium & & & & & \\
\hline \pm Titanium alloy & & & & & \\
\hline \pm tributyl tin & & & & & \\
\hline + tributyl tin hydride & & & & & \\
\hline \pm tributyl tin oxide & & & & & \\
\hline \pm Zinc Chloride & & & & & \\
\hline Record: $11 \mid 4 \Gamma$ & D|D|P* of 34 & 1 & & & I] \\
\hline Datasheet View & & & & & NUM $\square$ \\
\hline 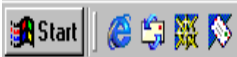 & 需Copy of Materialsdb : Data. & a. & 国Percent Dissociated:... & & 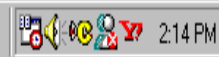 \\
\hline
\end{tabular}




\section{APPENDIX 18 - REACTIVITY/INSTABILITY TABLE}

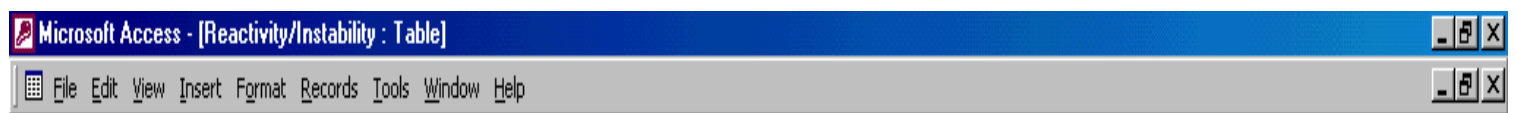

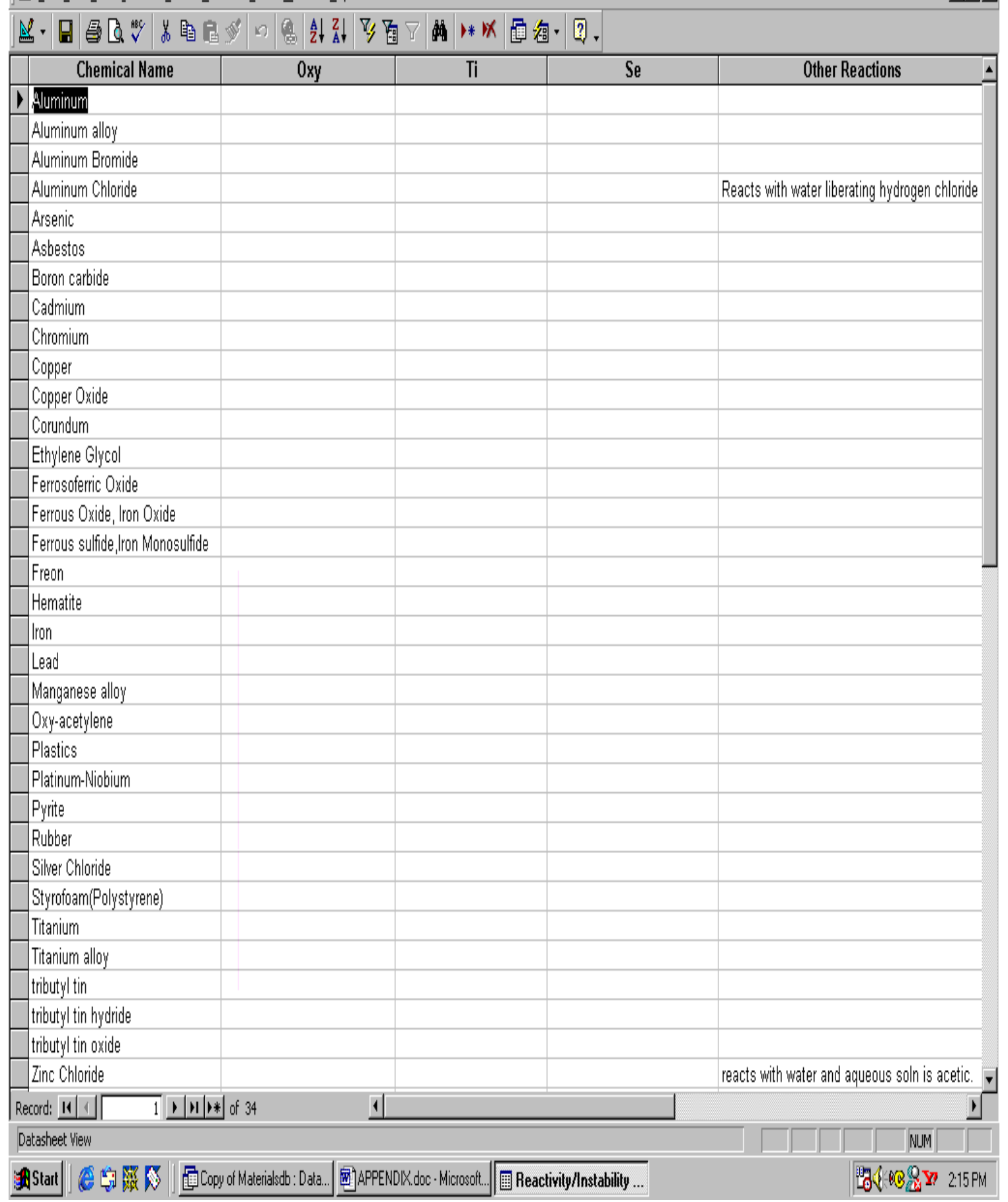




\section{APPENDIX 19 - TARGET ORAN APPLICABLE TABLE}

\section{Q Microsolt Access - [larget organ applicable : Table]}

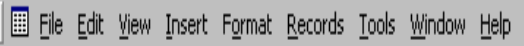

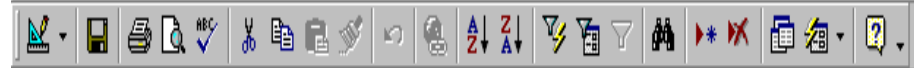

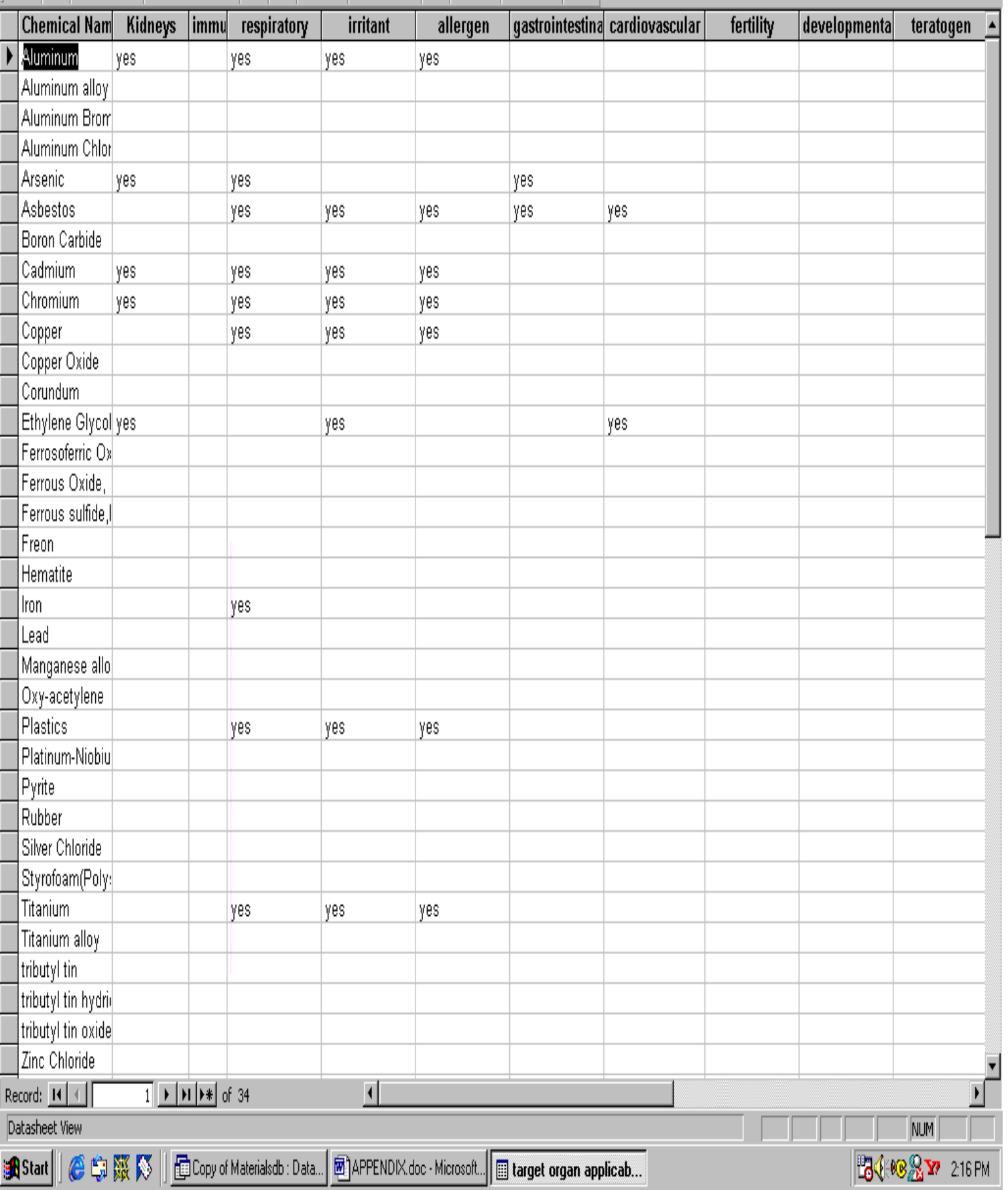




\section{APPENDIX 20 - TERRESTRIAL FATE TABLE}

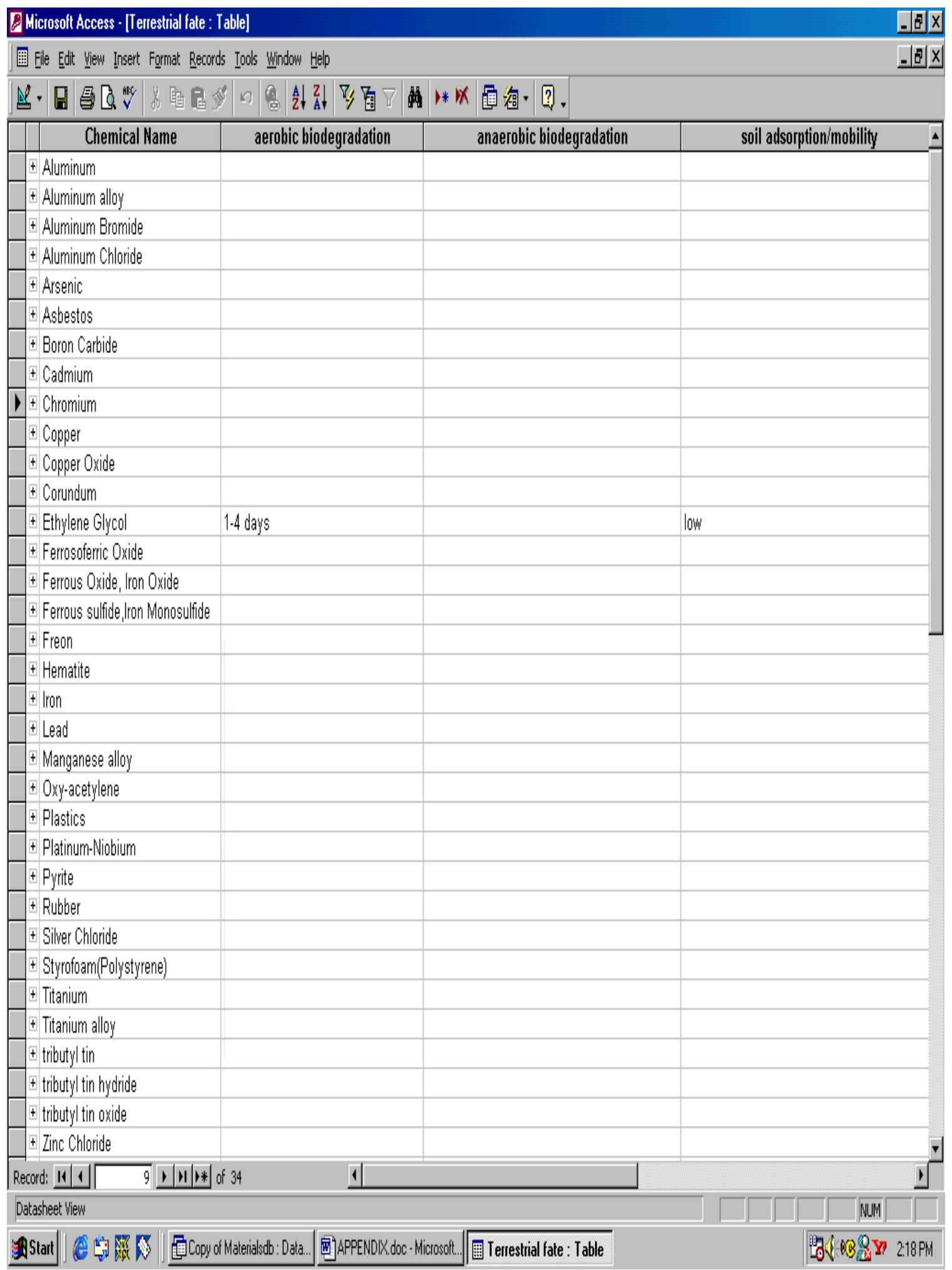




\section{APPENDIX 21 - WATER SOLUBILITY TABLE}

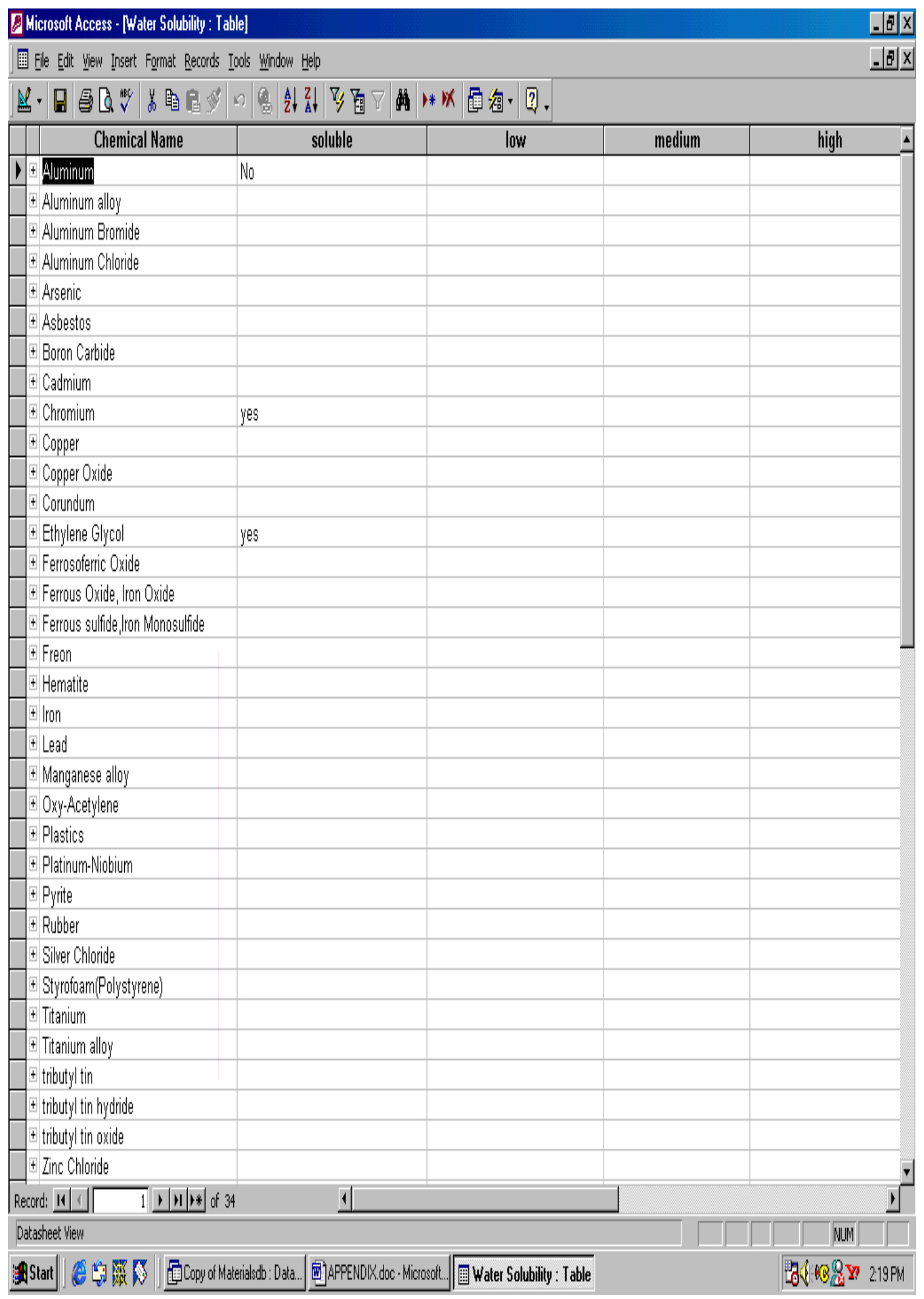




\section{APPENDIX 22 - WORKER EXPOSURE CRITERIA (CHRONIC- TWA) TABLE}

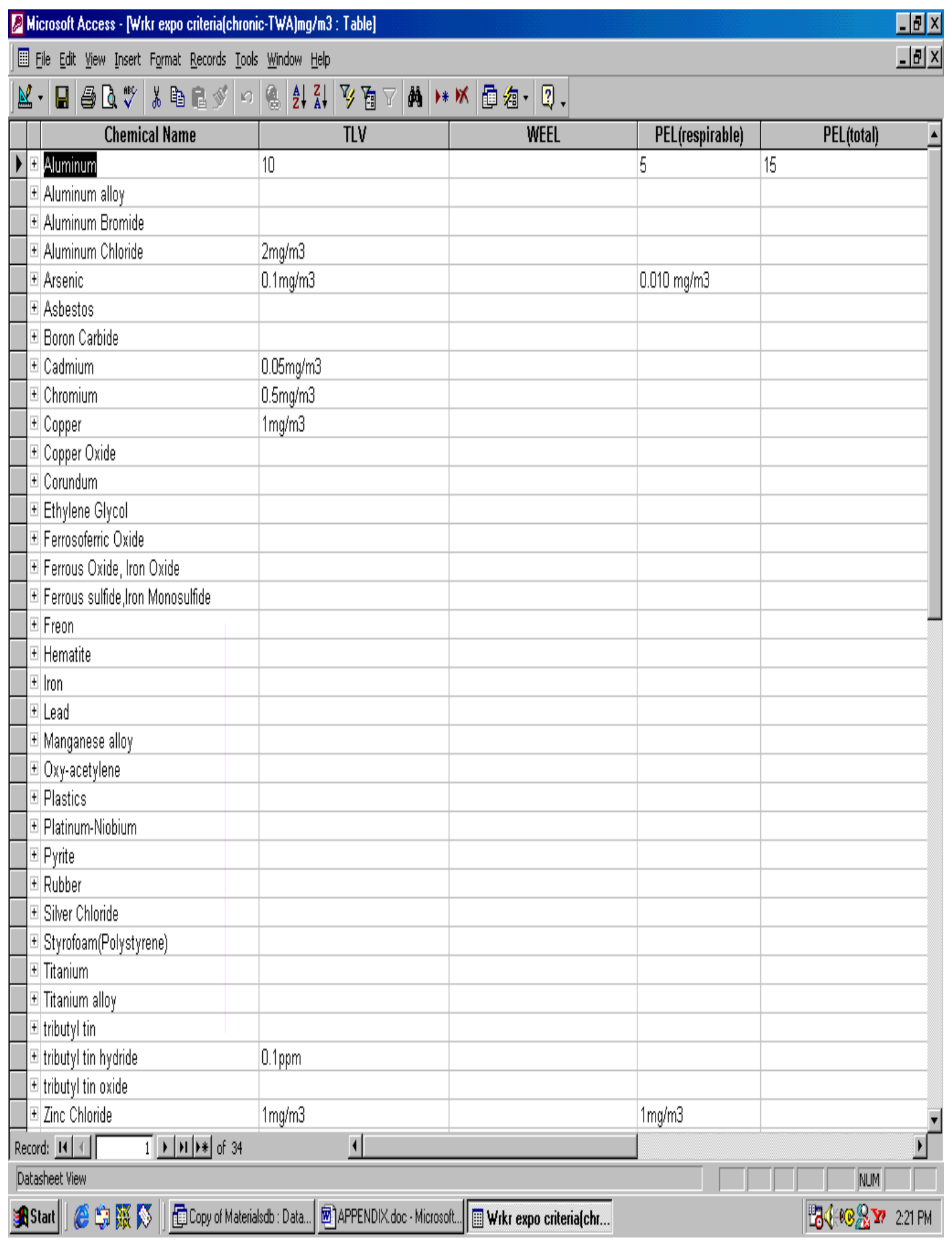




\section{APPENDIX 23 - WORKER EXPOSURE CRITERIA (ACUTE- CEIL) TABLE}

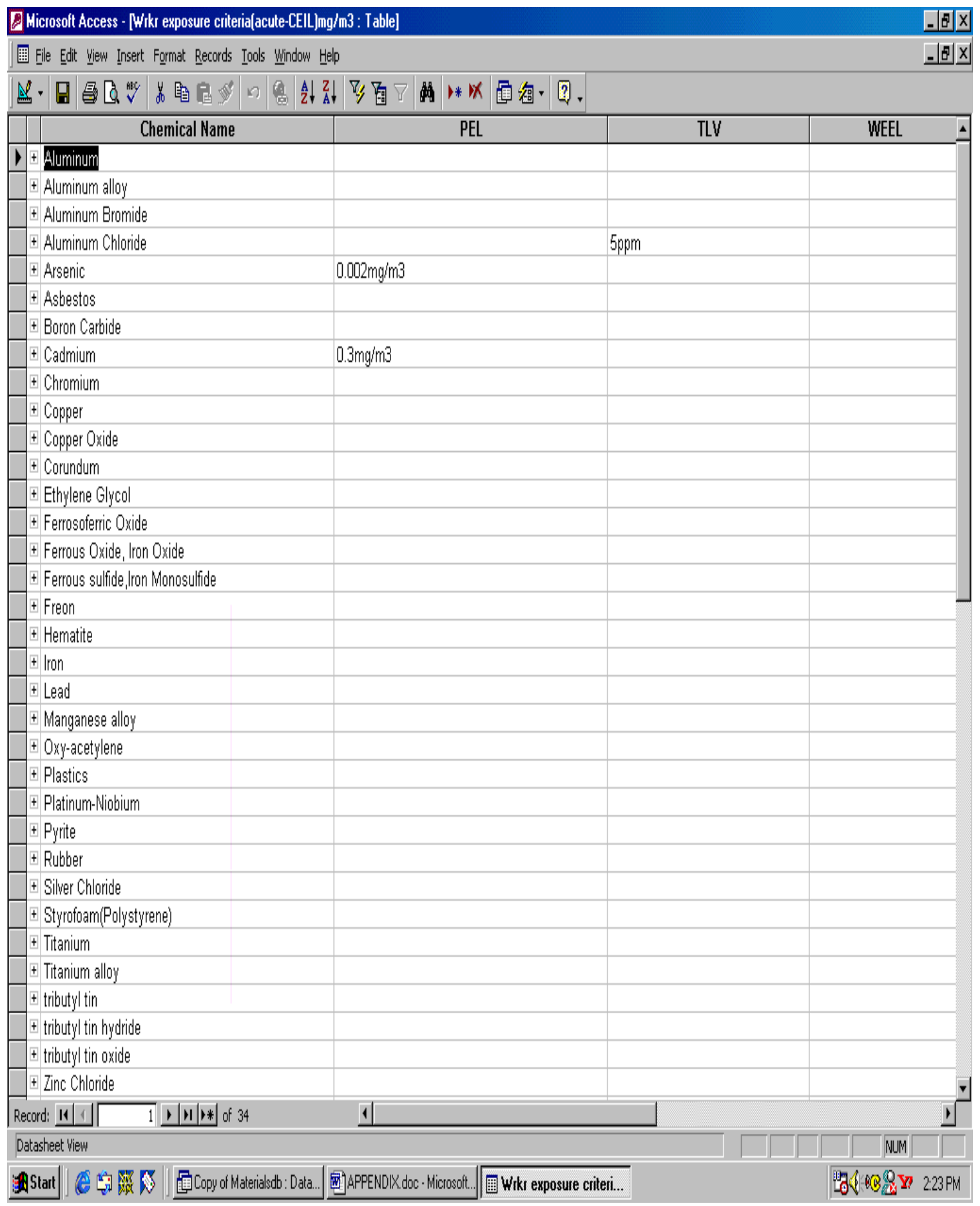




\section{APPENDIX 24 - WORKER EXPOSURE CRITERIA(ACUTE- STEL) TABLE}

\begin{tabular}{|c|c|c|}
\hline \multicolumn{3}{|c|}{ Q Microsolt Access - [Wikr exposure crileria(acule-STEL)mg/m3 : Table] } \\
\hline \multicolumn{3}{|l|}{ 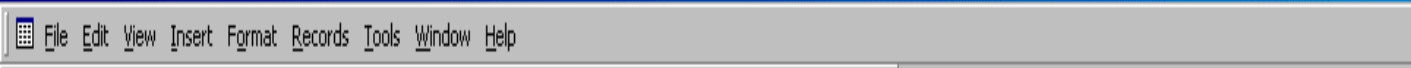 } \\
\hline \multicolumn{3}{|c|}{ 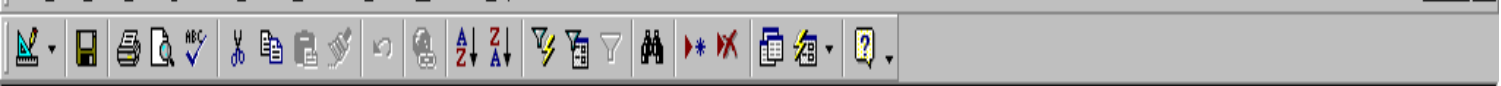 } \\
\hline \begin{tabular}{c|c|} 
& Chemical Name \\
\end{tabular} & TLV & WEEL \\
\hline \multicolumn{3}{|l|}{ I Aluminum } \\
\hline \multicolumn{3}{|l|}{$\mp$ Aluminum alloy } \\
\hline \multicolumn{3}{|l|}{ † Aluminum Bromide } \\
\hline \multicolumn{3}{|l|}{ † Aluminum Chloride } \\
\hline \pm Arsenic & $0.01 \mathrm{mg} / \mathrm{m} 3$ & \\
\hline \multicolumn{3}{|l|}{ \pm Asbestos } \\
\hline \multicolumn{3}{|l|}{ † Boron Carbide } \\
\hline \pm Cadmium & $0.1 \mathrm{mg} / \mathrm{m} 3$ & \\
\hline \multicolumn{3}{|l|}{ \pm Chromium } \\
\hline \multicolumn{3}{|l|}{ \pm Copper } \\
\hline \multicolumn{3}{|l|}{ + Copper Oxide } \\
\hline \multicolumn{3}{|l|}{ \pm Corundum } \\
\hline \multicolumn{3}{|l|}{ \pm Ethylene Glycol } \\
\hline \multicolumn{3}{|l|}{ † Ferrosoferric Oxide } \\
\hline \multicolumn{3}{|l|}{ † Ferrous Oxide, lron Oxide } \\
\hline \multicolumn{3}{|l|}{ † Ferrous sulfide, lron Monosulfide } \\
\hline \multicolumn{3}{|l|}{ t Freon } \\
\hline \multicolumn{3}{|l|}{ \pm † Hematite } \\
\hline \multicolumn{3}{|l|}{ † Iron } \\
\hline \multicolumn{3}{|l|}{ \pm t Lead } \\
\hline \multicolumn{3}{|l|}{ † Manganese alloy } \\
\hline \multicolumn{3}{|l|}{ \pm Oxy-acetylene } \\
\hline \multicolumn{3}{|l|}{ + Plastics } \\
\hline \multicolumn{3}{|l|}{ + Platinum-Niobium } \\
\hline \multicolumn{3}{|l|}{ † Pyyite } \\
\hline \multicolumn{3}{|l|}{ + Rubber } \\
\hline \pm Silver Chloride & & \\
\hline + Styrofoam(Polystyrene) & & \\
\hline \pm Titanium & & \\
\hline \pm Titanium alloy & & \\
\hline \pm tributyl tin & & \\
\hline t tributyl tin hydride & 0.2 & \\
\hline + tributyl tin oxide & & \\
\hline \pm Zinc Chloride & $2 \mathrm{mg} / \mathrm{m} 3$ & 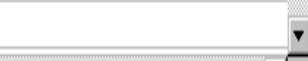 \\
\hline Record: $11|4 \longdiv { 1 }, 1, \operatorname{Ml}| * \mid$ of 34 & 1 & $1]$ \\
\hline Dotasheet Yiew & & $\square$ NMM $\square$ \\
\hline 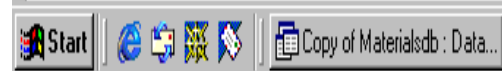 & 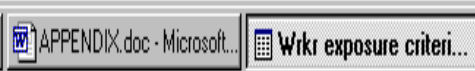 & 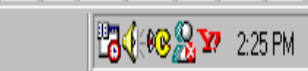 \\
\hline
\end{tabular}




\title{
APPENDIX - 25
}

\subsection{Acute Symptoms Code}

\author{
Private Sub Form_Unload(Cancel As Integer) \\ Screen. MousePointer $=$ vbDefault \\ End Sub
}

Private Sub datPrimaryRS_Error(ByVal ErrorNumber As Long, Description As String, ByVal Scode As Long, ByVal Source As String, ByVal HelpFile As String, ByVal HelpContext As Long, fCancelDisplay As Boolean)

'This is where you would put error handling code

'If you want to ignore errors, comment out the next line

'If you want to trap them, add code here to handle them

MsgBox "Data error event hit err:" \& Description

End Sub

Private Sub datPrimaryRS_MoveComplete(ByVal adReason As ADODB.EventReasonEnum, ByVal pError As

ADODB.Error, adStatus As ADODB.EventStatusEnum, ByVal pRecordset As ADODB.Recordset)

'This will display the current record position for this recordset

datPrimaryRS.Caption = "Record: " \& CStr(datPrimaryRS.Recordset.AbsolutePosition)

End Sub

Private Sub datPrimaryRS_WillChangeRecord(ByVal adReason As ADODB.EventReasonEnum, ByVal cRecords As Long, adStatus As ADODB.EventStatusEnum, ByVal pRecordset As ADODB.Recordset)

'This is where you put validation code

'This event gets called when the following actions occur

Dim bCancel As Boolean

Select Case adReason

Case adRsnAddNew

Case adRsnClose

Case adRsnDelete

Case adRsnFirstChange

Case adRsnMove

Case adRsnRequery

Case adRsnResynch

Case adRsnUndoAddNew

Case adRsnUndoDelete

Case adRsnUndoUpdate

Case adRsnUpdate

End Select

If bCancel Then adStatus $=$ adStatusCancel

End Sub

Private Sub cmdAdd_Click()

On Error GoTo AddErr

datPrimaryRS.Recordset.AddNew

Exit Sub

AddErr:

MsgBox Err.Description

End Sub 


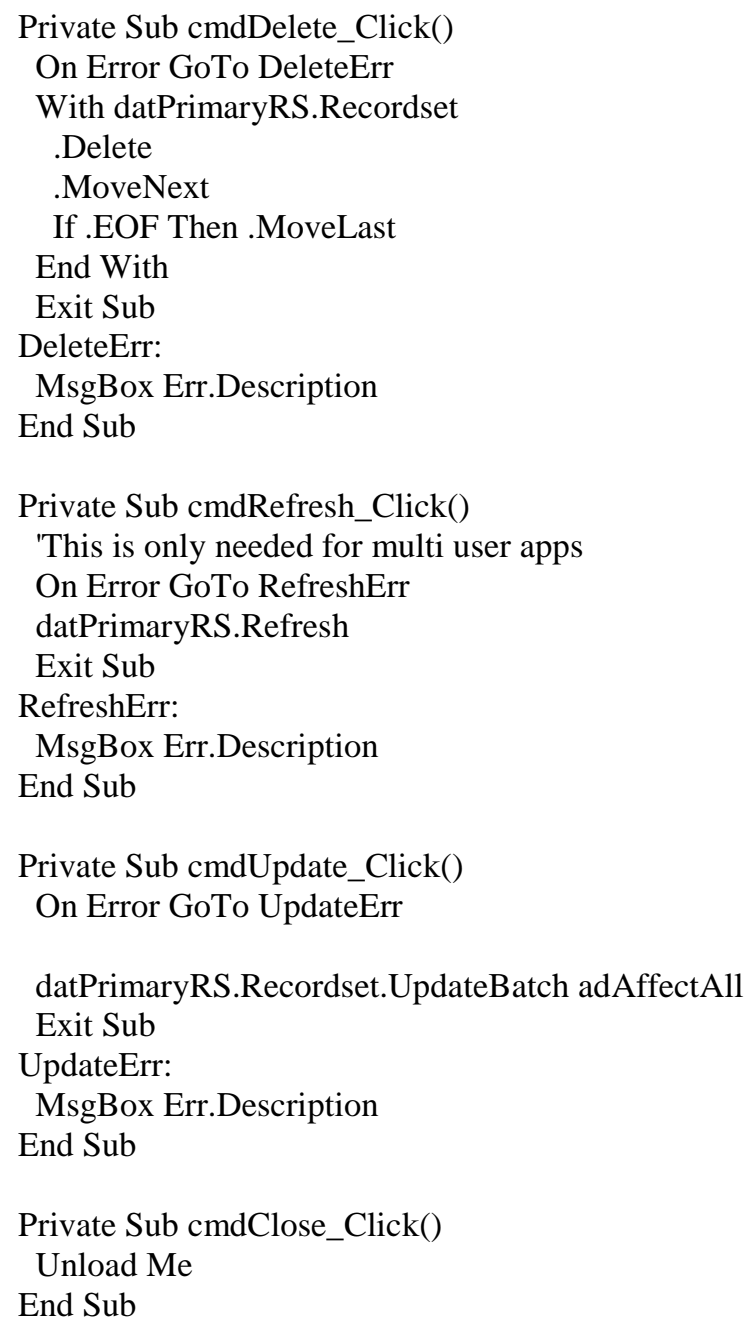

\subsection{Allergen form Code}

Private Sub Form_Unload(Cancel As Integer)

Screen. MousePointer $=$ vbDefault

End Sub

Private Sub datPrimaryRS_Error(ByVal ErrorNumber As Long, Description As String, ByVal Scode As Long, ByVal Source As String, ByVal HelpFile As String, ByVal HelpContext As Long, fCancelDisplay As Boolean) 'This is where you would put error handling code

'If you want to ignore errors, comment out the next line

'If you want to trap them, add code here to handle them

MsgBox "Data error event hit err:" \& Description

End Sub

Private Sub datPrimaryRS_MoveComplete(ByVal adReason As ADODB.EventReasonEnum, ByVal pError As ADODB.Error, adStatus As ADODB.EventStatusEnum, ByVal pRecordset As ADODB.Recordset)

'This will display the current record position for this recordset datPrimaryRS.Caption = "Record: " \& CStr(datPrimaryRS.Recordset.AbsolutePosition) End Sub 
Private Sub datPrimaryRS_WillChangeRecord(ByVal adReason As ADODB.EventReasonEnum, ByVal cRecords As Long, adStatus As ADODB.EventStatusEnum, ByVal pRecordset As ADODB.Recordset)

'This is where you put validation code

'This event gets called when the following actions occur

Dim bCancel As Boolean

Select Case adReason

Case adRsnAddNew

Case adRsnClose

Case adRsnDelete

Case adRsnFirstChange

Case adRsnMove

Case adRsnRequery

Case adRsnResynch

Case adRsnUndoAddNew

Case adRsnUndoDelete

Case adRsnUndoUpdate

Case adRsnUpdate

End Select

If bCancel Then adStatus = adStatusCancel

End Sub

Private Sub cmdAdd_Click()

On Error GoTo AddErr

datPrimaryRS.Recordset.AddNew

Exit Sub

AddErr:

MsgBox Err.Description

End Sub

Private Sub cmdDelete_Click()

On Error GoTo DeleteErr

With datPrimaryRS.Recordset

.Delete

.MoveNext

If .EOF Then .MoveLast

End With

Exit Sub

DeleteErr:

MsgBox Err.Description

End Sub

Private Sub cmdRefresh_Click()

'This is only needed for multi user apps

On Error GoTo RefreshErr

datPrimaryRS.Refresh

Exit Sub

RefreshErr:

MsgBox Err.Description

End Sub

Private Sub cmdUpdate_Click()

On Error GoTo UpdateErr 
datPrimaryRS.Recordset.UpdateBatch adAffectAll

Exit Sub

UpdateErr:

MsgBox Err.Description

End Sub

Private Sub cmdClose_Click()

Unload Me

End Sub

\subsection{Aquatic Fate form Code}

Private Sub Form_Unload(Cancel As Integer)

Screen.MousePointer $=$ vbDefault

End Sub

Private Sub datPrimaryRS_Error(ByVal ErrorNumber As Long, Description As String, ByVal Scode As Long, ByVal Source As String, ByVal HelpFile As String, ByVal HelpContext As Long, fCancelDisplay As Boolean)

'This is where you would put error handling code

'If you want to ignore errors, comment out the next line

'If you want to trap them, add code here to handle them

MsgBox "Data error event hit err:" \& Description

End Sub

Private Sub datPrimaryRS_MoveComplete(ByVal adReason As ADODB.EventReasonEnum, ByVal pError As ADODB.Error, adStatus As ADODB.EventStatusEnum, ByVal pRecordset As ADODB.Recordset)

'This will display the current record position for this recordset

datPrimaryRS.Caption = "Record: " \& CStr(datPrimaryRS.Recordset.AbsolutePosition)

End Sub

Private Sub datPrimaryRS_WillChangeRecord(ByVal adReason As ADODB.EventReasonEnum, ByVal cRecords As Long, adStatus As ADODB.EventStatusEnum, ByVal pRecordset As ADODB.Recordset)

'This is where you put validation code

'This event gets called when the following actions occur

Dim bCancel As Boolean

Select Case adReason

Case adRsnAddNew

Case adRsnClose

Case adRsnDelete

Case adRsnFirstChange

Case adRsnMove

Case adRsnRequery

Case adRsnResynch

Case adRsnUndoAddNew

Case adRsnUndoDelete

Case adRsnUndoUpdate

Case adRsnUpdate

End Select

If bCancel Then adStatus $=$ adStatusCancel

End Sub 


\title{
25.4 Atmospheric Fate form Code
}

\author{
Private Sub cmdAdd_Click() \\ On Error GoTo AddErr \\ datPrimaryRS.Recordset.AddNew \\ Exit Sub \\ AddErr: \\ MsgBox Err.Description \\ End Sub \\ Private Sub cmdDelete_Click() \\ On Error GoTo DeleteErr \\ With datPrimaryRS.Recordset \\ .Delete \\ .MoveNext \\ If .EOF Then .MoveLast \\ End With \\ Exit Sub \\ DeleteErr: \\ MsgBox Err.Description \\ End Sub \\ Private Sub cmdRefresh_Click() \\ 'This is only needed for multi user apps \\ On Error GoTo RefreshErr \\ datPrimaryRS.Refresh \\ Exit Sub \\ RefreshErr: \\ MsgBox Err.Description \\ End Sub \\ Private Sub cmdUpdate_Click() \\ On Error GoTo UpdateErr \\ datPrimaryRS.Recordset.UpdateBatch adAffectAll \\ Exit Sub \\ UpdateErr: \\ MsgBox Err.Description \\ End Sub \\ Private Sub cmdClose_Click() \\ Unload Me \\ End Sub
}

Private Sub Form_Unload(Cancel As Integer)

Screen.MousePointer $=$ vbDefault

End Sub

Private Sub datPrimaryRS_Error(ByVal ErrorNumber As Long, Description As String, ByVal Scode As Long, ByVal Source As String, ByVal HelpFile As String, ByVal HelpContext As Long, fCancelDisplay As Boolean)

'This is where you would put error handling code

'If you want to ignore errors, comment out the next line 
'If you want to trap them, add code here to handle them

MsgBox "Data error event hit err:" \& Description

End Sub

Private Sub datPrimaryRS_MoveComplete(ByVal adReason As ADODB.EventReasonEnum, ByVal pError As ADODB.Error, adStatus As ADODB.EventStatusEnum, ByVal pRecordset As ADODB.Recordset)

'This will display the current record position for this recordset

datPrimaryRS.Caption = "Record: " \& CStr(datPrimaryRS.Recordset.AbsolutePosition)

End Sub

Private Sub datPrimaryRS_WillChangeRecord(ByVal adReason As ADODB.EventReasonEnum, ByVal cRecords As Long, adStatus As ADODB.EventStatusEnum, ByVal pRecordset As ADODB.Recordset)

'This is where you put validation code

'This event gets called when the following actions occur

Dim bCancel As Boolean

Select Case adReason

Case adRsnAddNew

Case adRsnClose

Case adRsnDelete

Case adRsnFirstChange

Case adRsnMove

Case adRsnRequery

Case adRsnResynch

Case adRsnUndoAddNew

Case adRsnUndoDelete

Case adRsnUndoUpdate

Case adRsnUpdate

End Select

If $b$ Cancel Then adStatus $=$ adStatusCancel

End Sub

Private Sub cmdAdd_Click()

On Error GoTo AddErr

datPrimaryRS.Recordset.AddNew

Exit Sub

AddErr:

MsgBox Err.Description

End Sub

Private Sub cmdDelete_Click()

On Error GoTo DeleteErr

With datPrimaryRS.Recordset

.Delete

.MoveNext

If .EOF Then .MoveLast

End With

Exit Sub

DeleteErr:

MsgBox Err.Description

End Sub

Private Sub cmdRefresh_Click()

'This is only needed for multi user apps 


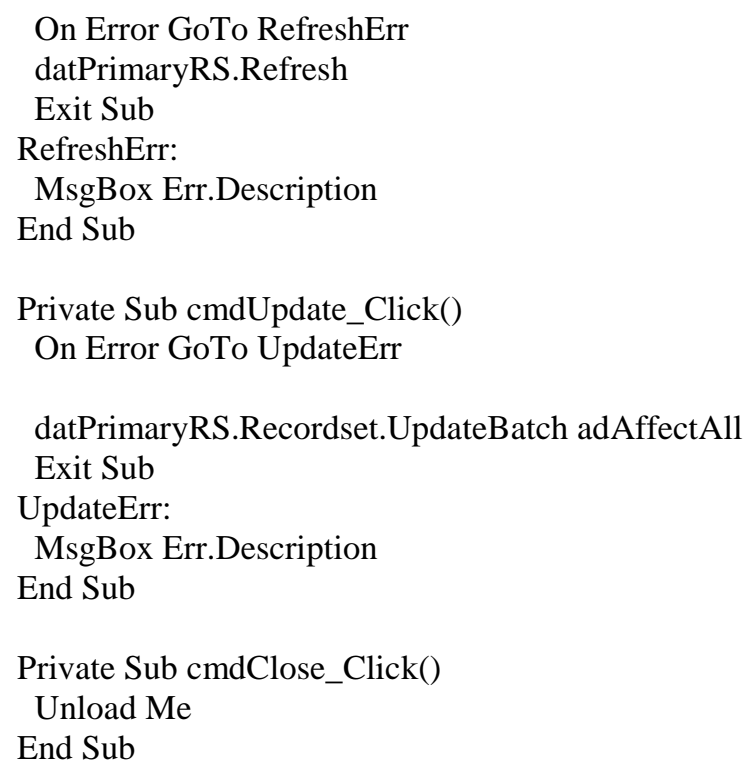

\subsection{BOD form Code :}

Private Sub Form_Unload(Cancel As Integer)

Screen.MousePointer $=$ vbDefault

End Sub

Private Sub datPrimaryRS_Error(ByVal ErrorNumber As Long, Description As String, ByVal Scode As Long, ByVal Source As String, ByVal HelpFile As String, ByVal HelpContext As Long, fCancelDisplay As Boolean)

'This is where you would put error handling code

'If you want to ignore errors, comment out the next line 'If you want to trap them, add code here to handle them

MsgBox "Data error event hit err:" \& Description

End Sub

Private Sub datPrimaryRS_MoveComplete(ByVal adReason As ADODB.EventReasonEnum, ByVal pError As ADODB.Error, adStatus As ADODB.EventStatusEnum, ByVal pRecordset As ADODB.Recordset)

'This will display the current record position for this recordset

datPrimaryRS.Caption = "Record: " \& CStr(datPrimaryRS.Recordset.AbsolutePosition)

End Sub

Private Sub datPrimaryRS_WillChangeRecord(ByVal adReason As ADODB.EventReasonEnum, ByVal cRecords As Long, adStatus As ADODB.EventStatusEnum, ByVal pRecordset As ADODB.Recordset)

'This is where you put validation code

'This event gets called when the following actions occur

Dim bCancel As Boolean

Select Case adReason

Case adRsnAddNew

Case adRsnClose

Case adRsnDelete

Case adRsnFirstChange

Case adRsnMove

Case adRsnRequery

Case adRsnResynch

Case adRsnUndoAddNew 


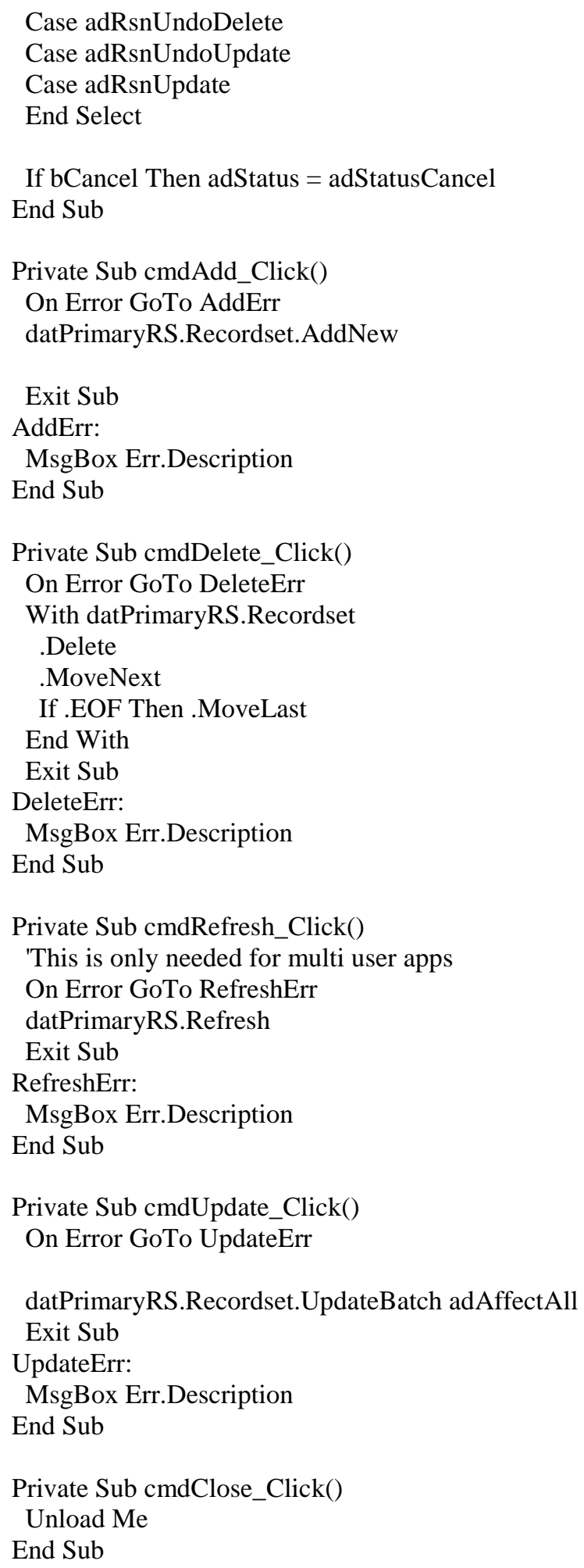

\subsection{Clean Air Act Code:}

rivate Sub Form_Unload(Cancel As Integer)

Screen.MousePointer $=$ vbDefault 


\section{End Sub}

Private Sub datPrimaryRS_Error(ByVal ErrorNumber As Long, Description As String, ByVal Scode As Long, ByVal Source As String, ByVal HelpFile As String, ByVal HelpContext As Long, fCancelDisplay As Boolean)

'This is where you would put error handling code

'If you want to ignore errors, comment out the next line

'If you want to trap them, add code here to handle them

MsgBox "Data error event hit err:" \& Description

End Sub

Private Sub datPrimaryRS_MoveComplete(ByVal adReason As ADODB.EventReasonEnum, ByVal pError As ADODB.Error, adStatus As ADODB.EventStatusEnum, ByVal pRecordset As ADODB.Recordset)

'This will display the current record position for this recordset

datPrimaryRS.Caption = "Record: " \& CStr(datPrimaryRS.Recordset.AbsolutePosition)

End Sub

Private Sub datPrimaryRS_WillChangeRecord(ByVal adReason As ADODB.EventReasonEnum, ByVal cRecords As Long, adStatus As ADODB.EventStatusEnum, ByVal pRecordset As ADODB.Recordset)

'This is where you put validation code

'This event gets called when the following actions occur

Dim bCancel As Boolean

Select Case adReason

Case adRsnAddNew

Case adRsnClose

Case adRsnDelete

Case adRsnFirstChange

Case adRsnMove

Case adRsnRequery

Case adRsnResynch

Case adRsnUndoAddNew

Case adRsnUndoDelete

Case adRsnUndoUpdate

Case adRsnUpdate

End Select

If $\mathrm{bCancel}$ Then adStatus $=$ adStatusCancel

End Sub

Private Sub cmdAdd_Click()

On Error GoTo AddErr

datPrimaryRS.Recordset.AddNew

Exit Sub

AddErr:

MsgBox Err.Description

End Sub

Private Sub cmdDelete_Click()

On Error GoTo DeleteErr

With datPrimaryRS.Recordset

.Delete

.MoveNext

If .EOF Then .MoveLast

End With

Exit Sub 


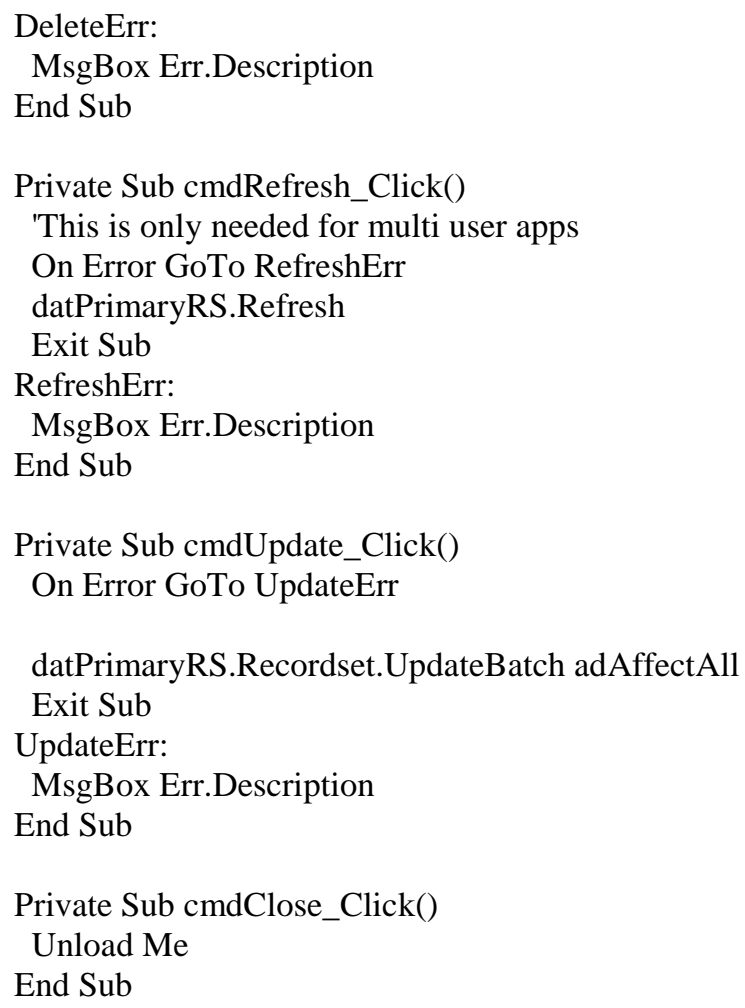

\subsection{Cancer form Code :}

Private Sub Form_Unload(Cancel As Integer)

Screen.MousePointer $=$ vbDefault

End Sub

Private Sub datPrimaryRS_Error(ByVal ErrorNumber As Long, Description As String, ByVal Scode As Long, ByVal Source As String, ByVal HelpFile As String, ByVal HelpContext As Long, fCancelDisplay As Boolean)

'This is where you would put error handling code

'If you want to ignore errors, comment out the next line

'If you want to trap them, add code here to handle them

MsgBox "Data error event hit err:" \& Description

End Sub

Private Sub datPrimaryRS_MoveComplete(ByVal adReason As ADODB.EventReasonEnum, ByVal pError As ADODB.Error, adStatus As ADODB.EventStatusEnum, ByVal pRecordset As ADODB.Recordset)

'This will display the current record position for this recordset

datPrimaryRS.Caption = "Record: " \& CStr(datPrimaryRS.Recordset.AbsolutePosition)

End Sub

Private Sub datPrimaryRS_WillChangeRecord(ByVal adReason As ADODB.EventReasonEnum, ByVal cRecords As Long, adStatus As ADODB.EventStatusEnum, ByVal pRecordset As ADODB.Recordset)

'This is where you put validation code

'This event gets called when the following actions occur

Dim bCancel As Boolean

Select Case adReason

Case adRsnAddNew

Case adRsnClose 
Case adRsnDelete

Case adRsnFirstChange

Case adRsnMove

Case adRsnRequery

Case adRsnResynch

Case adRsnUndoAddNew

Case adRsnUndoDelete

Case adRsnUndoUpdate

Case adRsnUpdate

End Select

If $b$ Cancel Then adStatus $=$ adStatusCancel

End Sub

Private Sub cmdAdd_Click()

On Error GoTo AddErr

datPrimaryRS.Recordset.AddNew

Exit Sub

AddErr:

MsgBox Err.Description

End Sub

Private Sub cmdDelete_Click()

On Error GoTo DeleteErr

With datPrimaryRS.Recordset

.Delete

.MoveNext

If .EOF Then .MoveLast

End With

Exit Sub

DeleteErr:

MsgBox Err.Description

End Sub

Private Sub cmdRefresh_Click()

'This is only needed for multi user apps

On Error GoTo RefreshErr

datPrimaryRS.Refresh

Exit Sub

RefreshErr:

MsgBox Err.Description

End Sub

Private Sub cmdUpdate_Click()

On Error GoTo UpdateErr

datPrimaryRS.Recordset.UpdateBatch adAffectAll

Exit Sub

UpdateErr:

MsgBox Err.Description

End Sub

Private Sub cmdClose_Click()

Unload Me

End Sub 


\subsection{Chronic symptoms form Code:}

Private Sub Form_Unload(Cancel As Integer)

Screen.MousePointer $=$ vbDefault

End Sub

Private Sub datPrimaryRS_Error(ByVal ErrorNumber As Long, Description As String, ByVal Scode As Long, ByVal Source As String, ByVal HelpFile As String, ByVal HelpContext As Long, fCancelDisplay As Boolean)

'This is where you would put error handling code

'If you want to ignore errors, comment out the next line

'If you want to trap them, add code here to handle them

MsgBox "Data error event hit err:" \& Description

End Sub

Private Sub datPrimaryRS_MoveComplete(ByVal adReason As ADODB.EventReasonEnum, ByVal pError As ADODB.Error, adStatus As ADODB.EventStatusEnum, ByVal pRecordset As ADODB.Recordset)

'This will display the current record position for this recordset

datPrimaryRS.Caption = "Record: " \& CStr(datPrimaryRS.Recordset.AbsolutePosition)

End Sub

Private Sub datPrimaryRS_WillChangeRecord(ByVal adReason As ADODB.EventReasonEnum, ByVal cRecords As Long, adStatus As ADODB.EventStatusEnum, ByVal pRecordset As ADODB.Recordset)

'This is where you put validation code

'This event gets called when the following actions occur

Dim bCancel As Boolean

Select Case adReason

Case adRsnAddNew

Case adRsnClose

Case adRsnDelete

Case adRsnFirstChange

Case adRsnMove

Case adRsnRequery

Case adRsnResynch

Case adRsnUndoAddNew

Case adRsnUndoDelete

Case adRsnUndoUpdate

Case adRsnUpdate

End Select

If $b$ Cancel Then adStatus $=$ adStatusCancel

End Sub

Private Sub cmdAdd_Click()

On Error GoTo AddErr

datPrimaryRS.Recordset.AddNew

Exit Sub

AddErr:

MsgBox Err.Description

End Sub

Private Sub cmdDelete_Click()

On Error GoTo DeleteErr

With datPrimaryRS.Recordset

.Delete 


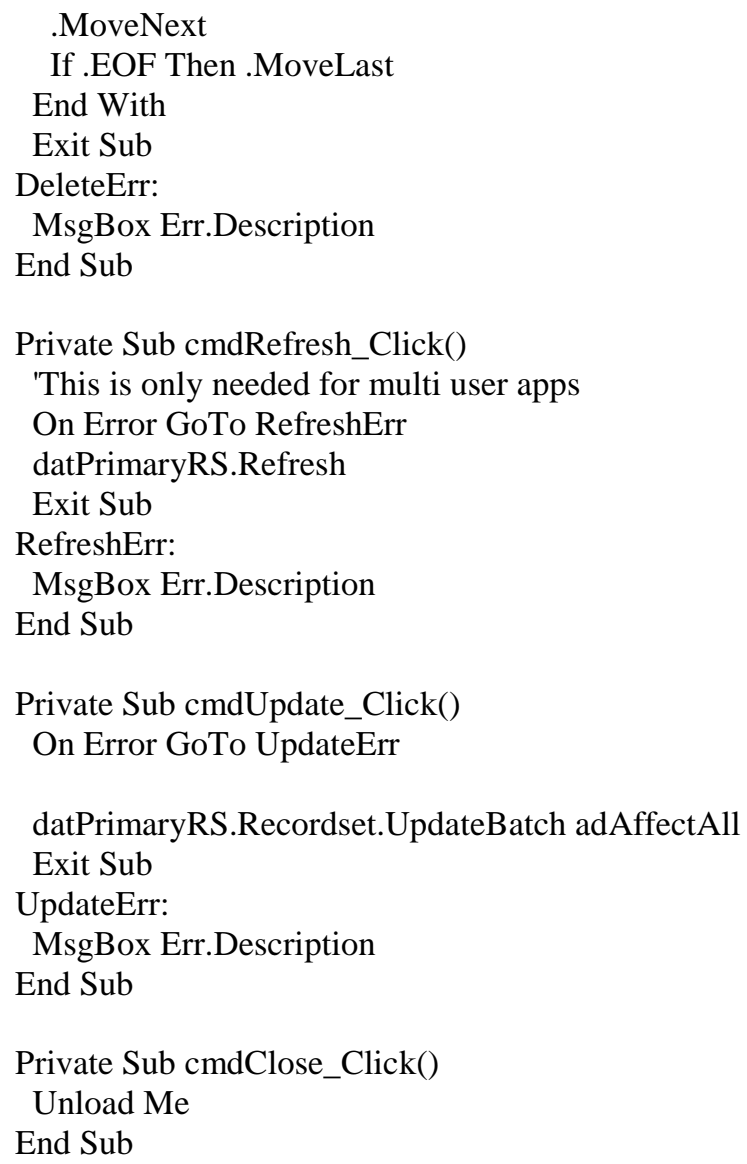

\subsection{Corrosivity form Code}

Private Sub Form_Unload(Cancel As Integer)

Screen.MousePointer $=$ vbDefault

End Sub

Private Sub datPrimaryRS_Error(ByVal ErrorNumber As Long, Description As String, ByVal Scode As Long, ByVal Source As String, ByVal HelpFile As String, ByVal HelpContext As Long, fCancelDisplay As Boolean)

'This is where you would put error handling code

'If you want to ignore errors, comment out the next line

'If you want to trap them, add code here to handle them

MsgBox "Data error event hit err:" \& Description

End Sub

Private Sub datPrimaryRS_MoveComplete(ByVal adReason As ADODB.EventReasonEnum, ByVal pError As ADODB.Error, adStatus As ADODB.EventStatusEnum, ByVal pRecordset As ADODB.Recordset)

'This will display the current record position for this recordset

datPrimaryRS.Caption = "Record: " \& CStr(datPrimaryRS.Recordset.AbsolutePosition)

End Sub

Private Sub datPrimaryRS_WillChangeRecord(ByVal adReason As ADODB.EventReasonEnum, ByVal cRecords As Long, adStatus As ADODB.EventStatusEnum, ByVal pRecordset As ADODB.Recordset)

'This is where you put validation code

'This event gets called when the following actions occur

Dim bCancel As Boolean 
Select Case adReason

Case adRsnAddNew

Case adRsnClose

Case adRsnDelete

Case adRsnFirstChange

Case adRsnMove

Case adRsnRequery

Case adRsnResynch

Case adRsnUndoAddNew

Case adRsnUndoDelete

Case adRsnUndoUpdate

Case adRsnUpdate

End Select

If bCancel Then adStatus $=$ adStatusCancel

End Sub

Private Sub cmdAdd_Click()

On Error GoTo AddErr

datPrimaryRS.Recordset.AddNew

Exit Sub

AddErr:

MsgBox Err.Description

End Sub

Private Sub cmdDelete_Click()

On Error GoTo DeleteErr

With datPrimaryRS.Recordset

.Delete

MoveNext

If .EOF Then .MoveLast

End With

Exit Sub

DeleteErr:

MsgBox Err.Description

End Sub

Private Sub cmdRefresh_Click()

'This is only needed for multi user apps

On Error GoTo RefreshErr

datPrimaryRS.Refresh

Exit Sub

RefreshErr:

MsgBox Err.Description

End Sub

Private Sub cmdUpdate_Click()

On Error GoTo UpdateErr

datPrimaryRS.Recordset.UpdateBatch adAffectAll

Exit Sub

UpdateErr:

MsgBox Err.Description

End Sub 
Private Sub cmdClose_Click()

Unload Me

End Sub

\subsection{Characteristic RCRA form Code :}

Private Sub Form_Unload(Cancel As Integer)

Screen.MousePointer $=$ vbDefault

End Sub

Private Sub datPrimaryRS_Error(ByVal ErrorNumber As Long, Description As String, ByVal Scode As Long, ByVal Source As String, ByVal HelpFile As String, ByVal HelpContext As Long, fCancelDisplay As Boolean)

'This is where you would put error handling code

'If you want to ignore errors, comment out the next line

'If you want to trap them, add code here to handle them

MsgBox "Data error event hit err:" \& Description

End Sub

Private Sub datPrimaryRS_MoveComplete(ByVal adReason As ADODB.EventReasonEnum, ByVal pError As

ADODB.Error, adStatus As ADODB.EventStatusEnum, ByVal pRecordset As ADODB.Recordset)

'This will display the current record position for this recordset

datPrimaryRS.Caption = "Record: " \& CStr(datPrimaryRS.Recordset.AbsolutePosition)

End Sub

Private Sub datPrimaryRS_WillChangeRecord(ByVal adReason As ADODB.EventReasonEnum, ByVal cRecords As Long, adStatus As ADODB.EventStatusEnum, ByVal pRecordset As ADODB.Recordset)

'This is where you put validation code

'This event gets called when the following actions occur

Dim bCancel As Boolean

Select Case adReason

Case adRsnAddNew

Case adRsnClose

Case adRsnDelete

Case adRsnFirstChange

Case adRsnMove

Case adRsnRequery

Case adRsnResynch

Case adRsnUndoAddNew

Case adRsnUndoDelete

Case adRsnUndoUpdate

Case adRsnUpdate

End Select

If $b$ Cancel Then adStatus $=$ adStatusCancel

End Sub

Private Sub cmdAdd_Click()

On Error GoTo AddErr

datPrimaryRS.Recordset.AddNew

Exit Sub

AddErr:

MsgBox Err.Description 


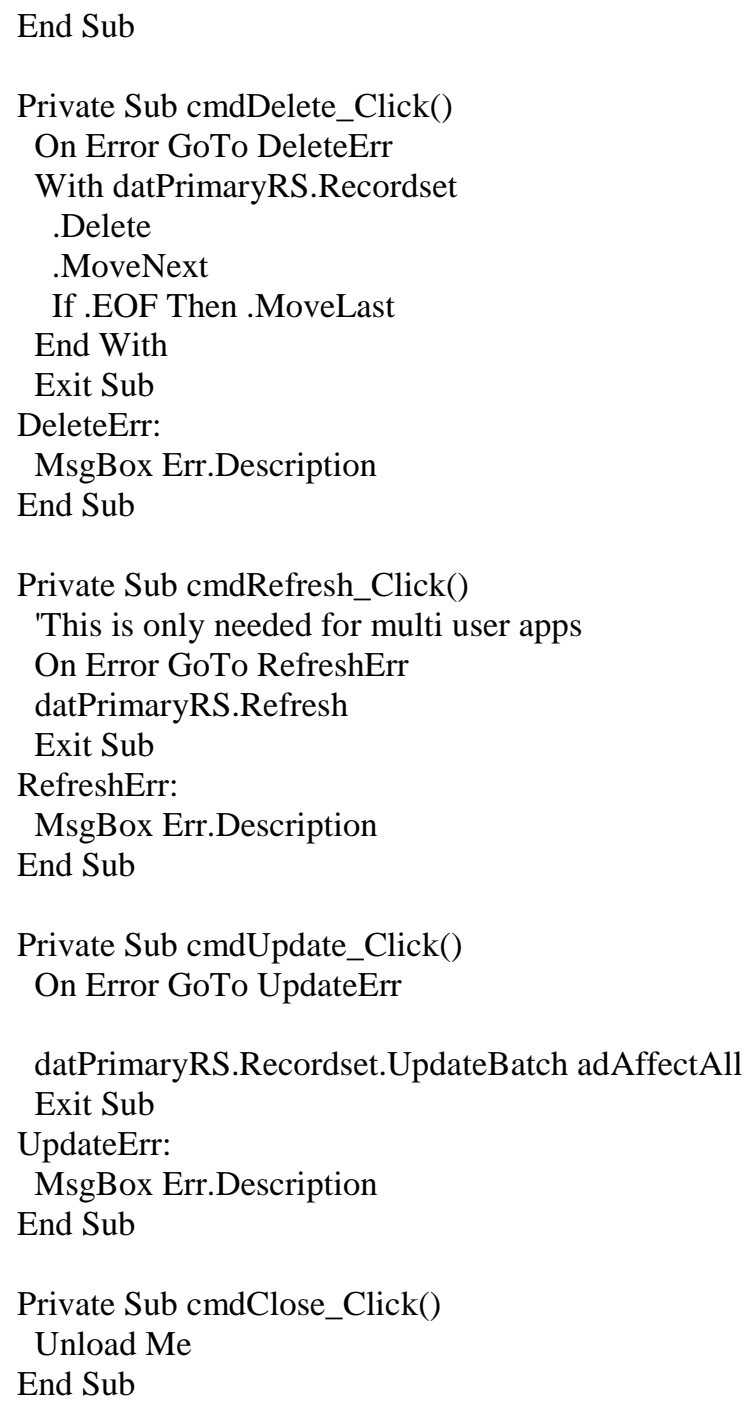

\subsection{DOT Code}

Private Sub Form_Unload(Cancel As Integer)

Screen.MousePointer $=$ vbDefault

End Sub

Private Sub datPrimaryRS_Error(ByVal ErrorNumber As Long, Description As String, ByVal Scode As Long, ByVal Source As String, ByVal HelpFile As String, ByVal HelpContext As Long, fCancelDisplay As Boolean)

'This is where you would put error handling code

'If you want to ignore errors, comment out the next line

'If you want to trap them, add code here to handle them

MsgBox "Data error event hit err:" \& Description

End Sub

Private Sub datPrimaryRS_MoveComplete(ByVal adReason As ADODB.EventReasonEnum, ByVal pError As ADODB.Error, adStatus As ADODB.EventStatusEnum, ByVal pRecordset As ADODB.Recordset)

'This will display the current record position for this recordset datPrimaryRS.Caption = "Record: " \& CStr(datPrimaryRS.Recordset.AbsolutePosition)

End Sub 
Private Sub datPrimaryRS_WillChangeRecord(ByVal adReason As ADODB.EventReasonEnum, ByVal cRecords As Long, adStatus As ADODB.EventStatusEnum, ByVal pRecordset As ADODB.Recordset)

'This is where you put validation code

'This event gets called when the following actions occur

Dim bCancel As Boolean

Select Case adReason

Case adRsnAddNew

Case adRsnClose

Case adRsnDelete

Case adRsnFirstChange

Case adRsnMove

Case adRsnRequery

Case adRsnResynch

Case adRsnUndoAddNew

Case adRsnUndoDelete

Case adRsnUndoUpdate

Case adRsnUpdate

End Select

If bCancel Then adStatus = adStatusCancel

End Sub

Private Sub cmdAdd_Click()

On Error GoTo AddErr

datPrimaryRS.Recordset.AddNew

Exit Sub

AddErr:

MsgBox Err.Description

End Sub

Private Sub cmdDelete_Click()

On Error GoTo DeleteErr

With datPrimaryRS.Recordset

.Delete

.MoveNext

If .EOF Then .MoveLast

End With

Exit Sub

DeleteErr:

MsgBox Err.Description

End Sub

Private Sub cmdRefresh_Click()

'This is only needed for multi user apps

On Error GoTo RefreshErr

datPrimaryRS.Refresh

Exit Sub

RefreshErr:

MsgBox Err.Description

End Sub

Private Sub cmdUpdate_Click()

On Error GoTo UpdateErr 
datPrimaryRS.Recordset.UpdateBatch adAffectAll

Exit Sub

UpdateErr:

MsgBox Err.Description

End Sub

Private Sub cmdClose_Click()

Unload Me

End Sub

\subsection{Extinguishing Agents form Code}

Private Sub Form_Unload(Cancel As Integer)

Screen.MousePointer $=$ vbDefault

End Sub

Private Sub datPrimaryRS_Error(ByVal ErrorNumber As Long, Description As String, ByVal Scode As Long, ByVal Source As String, ByVal HelpFile As String, ByVal HelpContext As Long, fCancelDisplay As Boolean)

'This is where you would put error handling code

'If you want to ignore errors, comment out the next line

'If you want to trap them, add code here to handle them

MsgBox "Data error event hit err:" \& Description

End Sub

Private Sub datPrimaryRS_MoveComplete(ByVal adReason As ADODB.EventReasonEnum, ByVal pError As ADODB.Error, adStatus As ADODB.EventStatusEnum, ByVal pRecordset As ADODB.Recordset)

'This will display the current record position for this recordset

datPrimaryRS.Caption = "Record: " \& CStr(datPrimaryRS.Recordset.AbsolutePosition)

End Sub

Private Sub datPrimaryRS_WillChangeRecord(ByVal adReason As ADODB.EventReasonEnum, ByVal cRecords As Long, adStatus As ADODB.EventStatusEnum, ByVal pRecordset As ADODB.Recordset)

'This is where you put validation code

'This event gets called when the following actions occur

Dim bCancel As Boolean

Select Case adReason

Case adRsnAddNew

Case adRsnClose

Case adRsnDelete

Case adRsnFirstChange

Case adRsnMove

Case adRsnRequery

Case adRsnResynch

Case adRsnUndoAddNew

Case adRsnUndoDelete

Case adRsnUndoUpdate

Case adRsnUpdate

End Select

If $b$ Cancel Then adStatus $=$ adStatusCancel

End Sub

Private Sub cmdAdd_Click() 


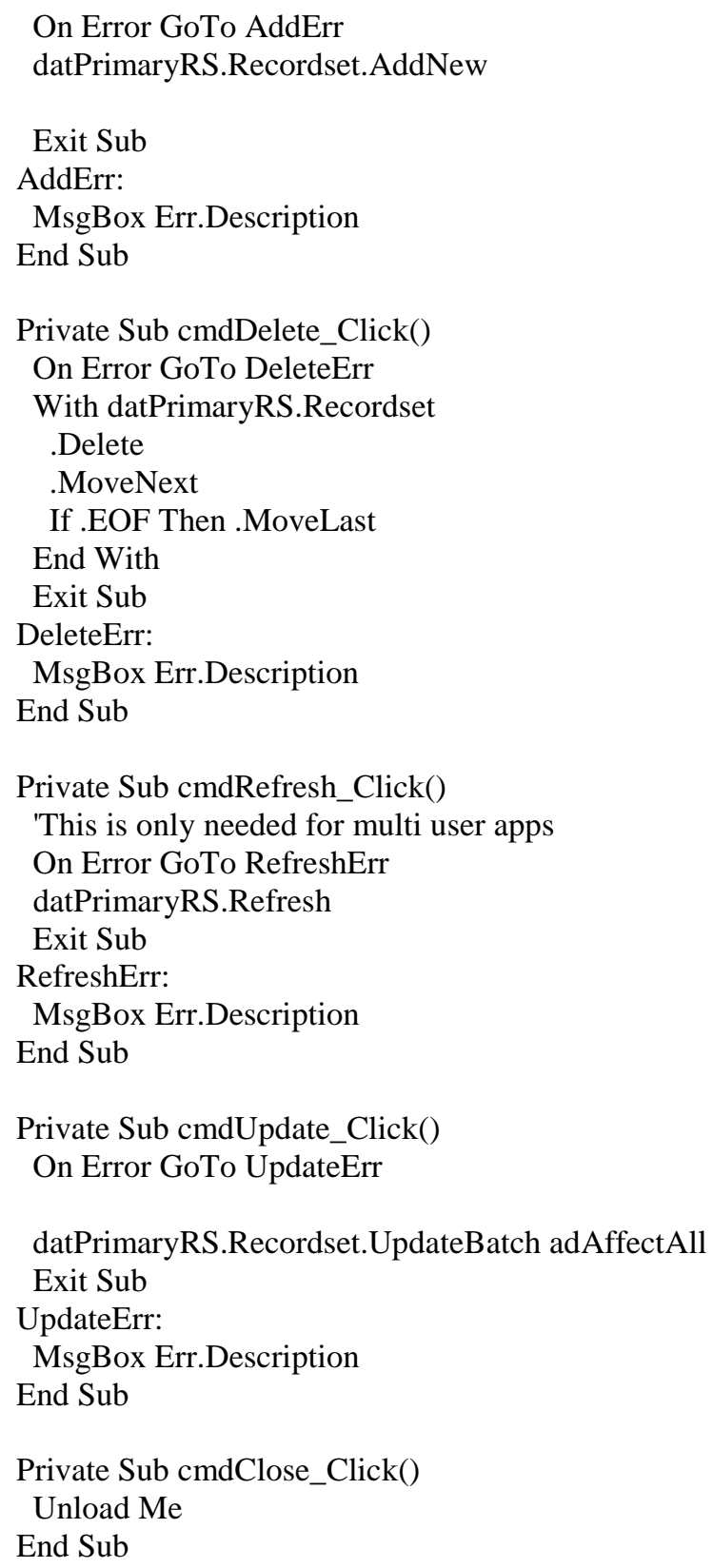

\subsection{File Maintenance form Code:}

Private Sub Form_Unload(Cancel As Integer)

Screen.MousePointer $=$ vbDefault

End Sub

Private Sub datPrimaryRS_Error(ByVal ErrorNumber As Long, Description As String, ByVal Scode As Long, ByVal Source As String, ByVal HelpFile As String, ByVal HelpContext As Long, fCancelDisplay As Boolean)

'This is where you would put error handling code

'If you want to ignore errors, comment out the next line

'If you want to trap them, add code here to handle them

MsgBox "Data error event hit err:" \& Description

End Sub 
Private Sub datPrimaryRS_MoveComplete(ByVal adReason As ADODB.EventReasonEnum, ByVal pError As ADODB.Error, adStatus As ADODB.EventStatusEnum, ByVal pRecordset As ADODB.Recordset)

'This will display the current record position for this recordset

datPrimaryRS.Caption = "Record: " \& CStr(datPrimaryRS.Recordset.AbsolutePosition)

End Sub

Private Sub datPrimaryRS_WillChangeRecord(ByVal adReason As ADODB.EventReasonEnum, ByVal cRecords As Long, adStatus As ADODB.EventStatusEnum, ByVal pRecordset As ADODB.Recordset)

'This is where you put validation code

'This event gets called when the following actions occur

Dim bCancel As Boolean

Select Case adReason

Case adRsnAddNew

Case adRsnClose

Case adRsnDelete

Case adRsnFirstChange

Case adRsnMove

Case adRsnRequery

Case adRsnResynch

Case adRsnUndoAddNew

Case adRsnUndoDelete

Case adRsnUndoUpdate

Case adRsnUpdate

End Select

If bCancel Then adStatus = adStatusCancel

End Sub

Private Sub cmdAdd_Click()

On Error GoTo AddErr

datPrimaryRS.Recordset.AddNew

Exit Sub

AddErr:

MsgBox Err.Description

End Sub

Private Sub cmdDelete_Click()

On Error GoTo DeleteErr

With datPrimaryRS.Recordset

.Delete

.MoveNext

If .EOF Then .MoveLast

End With

Exit Sub

DeleteErr:

MsgBox Err.Description

End Sub

Private Sub cmdRefresh_Click()

'This is only needed for multi user apps

On Error GoTo RefreshErr

datPrimaryRS.Refresh

Exit Sub 


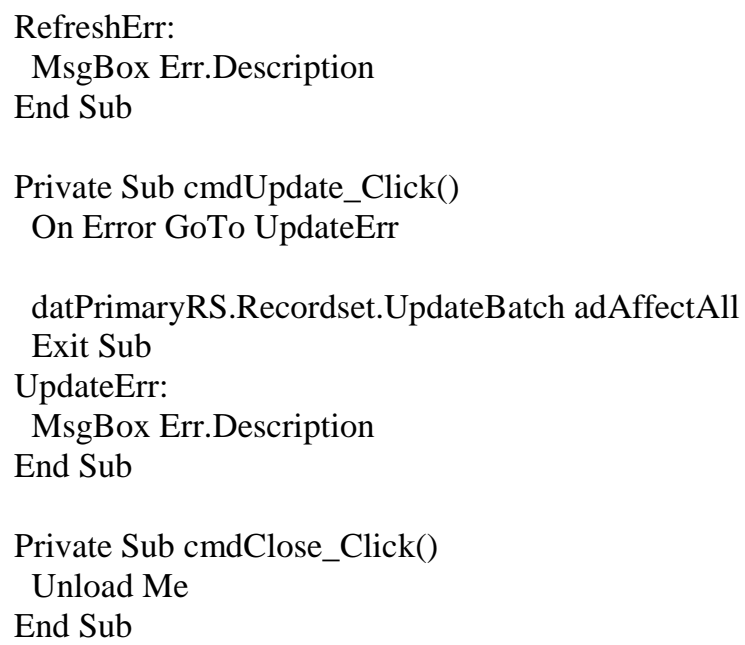

\subsection{Flammability/Combustibility form Code}

Private Sub Form_Unload(Cancel As Integer)

Screen.MousePointer $=$ vbDefault

End Sub

Private Sub datPrimaryRS_Error(ByVal ErrorNumber As Long, Description As String, ByVal Scode As Long, ByVal Source As String, ByVal HelpFile As String, ByVal HelpContext As Long, fCancelDisplay As Boolean)

'This is where you would put error handling code

'If you want to ignore errors, comment out the next line

'If you want to trap them, add code here to handle them

MsgBox "Data error event hit err:" \& Description

End Sub

Private Sub datPrimaryRS_MoveComplete(ByVal adReason As ADODB.EventReasonEnum, ByVal pError As ADODB.Error, adStatus As ADODB.EventStatusEnum, ByVal pRecordset As ADODB.Recordset)

'This will display the current record position for this recordset datPrimaryRS.Caption = "Record: " \& CStr(datPrimaryRS.Recordset.AbsolutePosition)

End Sub

Private Sub datPrimaryRS_WillChangeRecord(ByVal adReason As ADODB.EventReasonEnum, ByVal cRecords As Long, adStatus As ADODB.EventStatusEnum, ByVal pRecordset As ADODB.Recordset)

'This is where you put validation code

'This event gets called when the following actions occur

Dim bCancel As Boolean

Select Case adReason

Case adRsnAddNew

Case adRsnClose

Case adRsnDelete

Case adRsnFirstChange

Case adRsnMove

Case adRsnRequery

Case adRsnResynch

Case adRsnUndoAddNew

Case adRsnUndoDelete

Case adRsnUndoUpdate

Case adRsnUpdate 


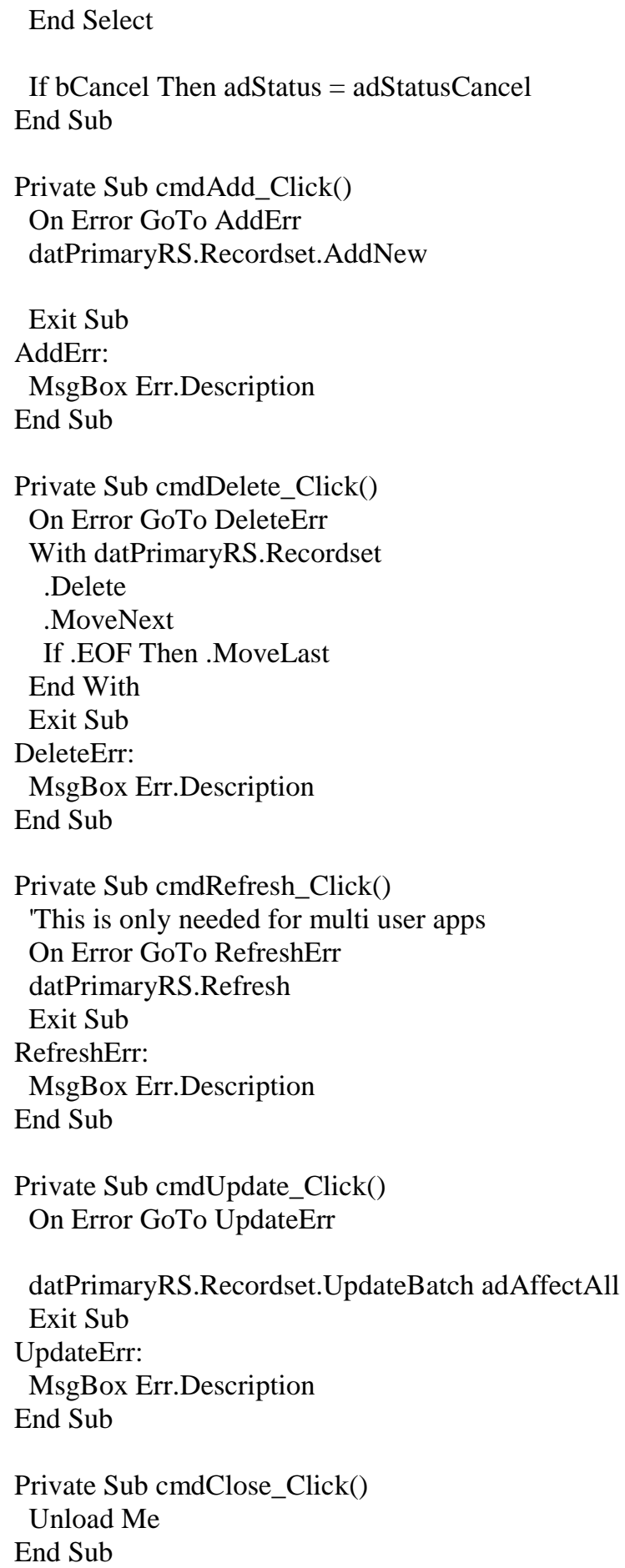

\subsection{Main Form Code:}

Private Sub AC_Click()

frmacutesymptoms. Show 1

End Sub

Private Sub AF_Click() 
frmaquaticfate. Show 1

End Sub

Private Sub allergen_Click()

frmallergen.Show 1

End Sub

Private Sub ATF_Click()

frmatmfate. Show 1

End Sub

Private Sub bod_Click()

frmbod. Show 1

End Sub

Private Sub Boilingpoint_Click()

frmquerybp. Show 1

End Sub

Private Sub CAA_Click()

frmcaa.Show 1

End Sub

Private Sub cancer_Click()

frmcancer.Show 1

End Sub

Private Sub casno_Click()

frmsearchcsno. Show 1

End Sub

Private Sub chemicalname_Click()

frmsearchchname. Show 1

End Sub

Private Sub Co_Click()

frmcorrosivity. Show 1

End Sub

Private Sub CRCRA_Click()

frmcrcra.Show 1

End Sub

Private Sub CS_Click()

frmchronicsymptoms. Show 1

End Sub

Private Sub DOt_Click()

frmdot.Show 1

End Sub

Private Sub Ea_Click()

frmextinguishingagents. Show 1

End Sub

Private Sub exit_Click() 


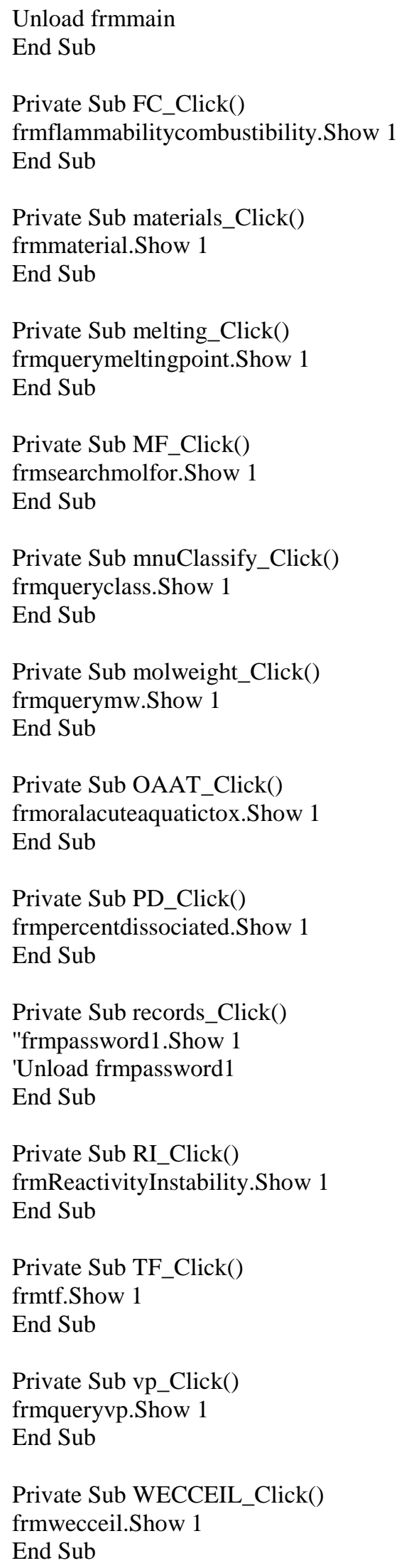


Private Sub WECSTEL_Click()

frmwecstel.Show 1

End Sub

Private Sub WECTWA_Click()

frmwectwa. Show 1

End Sub

Private Sub WL_Click()

End Sub

Private Sub WS_Click()

frmwatersolubility.Show 1

End Sub

\subsection{Materials form Code}

Private Sub Form_Unload(Cancel As Integer)

Screen .MousePointer $=$ vbDefault

End Sub

Private Sub datPrimaryRS_Error(ByVal ErrorNumber As Long, Description As String, ByVal Scode As Long, ByVal Source As String, ByVal HelpFile As String, ByVal HelpContext As Long, fCancelDisplay As Boolean)

This is where you would put error handling code

'If you want to ignore errors, comment out the next line

'If you want to trap them, add code here to handle them

MsgBox "Data error event hit err:" \& Description

End Sub

Private Sub datPrimaryRS_MoveComplete(ByVal adReason As ADODB.EventReasonEnum, ByVal pError As

ADODB.Error, adStatus As ADODB.EventStatusEnum, ByVal pRecordset As ADODB.Recordset)

'This will display the current record position for this recordset

datPrimaryRS.Caption = "Record: " \& CStr(datPrimaryRS.Recordset.AbsolutePosition)

End Sub

Private Sub datPrimaryRS_WillChangeRecord(ByVal adReason As ADODB.EventReasonEnum, ByVal cRecords As Long, adStatus As ADODB.EventStatusEnum, ByVal pRecordset As ADODB.Recordset)

'This is where you put validation code

'This event gets called when the following actions occur

Dim bCancel As Boolean

Select Case adReason

Case adRsnAddNew

Case adRsnClose

Case adRsnDelete

Case adRsnFirstChange

Case adRsnMove

Case adRsnRequery

Case adRsnResynch

Case adRsnUndoAddNew

Case adRsnUndoDelete

Case adRsnUndoUpdate

Case adRsnUpdate

End Select 
If bCancel Then adStatus $=$ adStatusCancel

End Sub

Private Sub cmdAdd_Click()

On Error GoTo AddErr

datPrimaryRS.Recordset.AddNew

Exit Sub

AddErr:

MsgBox Err.Description

End Sub

Private Sub cmdDelete_Click()

On Error GoTo DeleteErr

With datPrimaryRS.Recordset

.Delete

.MoveNext

If .EOF Then .MoveLast

End With

Exit Sub

DeleteErr:

MsgBox Err.Description

End Sub

Private Sub cmdRefresh_Click()

'This is only needed for multi user apps

On Error GoTo RefreshErr

datPrimaryRS.Refresh

Exit Sub

RefreshErr:

MsgBox Err.Description

End Sub

Private Sub cmdUpdate_Click()

'On Error GoTo UpdateErr

datPrimaryRS.Recordset.UpdateBatch adAffectAll

' Exit Sub

'UpdateErr:

'MsgBox Err.Description

End Sub

Private Sub cmdClose_Click()

Unload Me

End Sub

\subsection{Oral Acute aquatic Tox Form - code}

Private Sub Form_Unload(Cancel As Integer)

Screen.MousePointer $=$ vbDefault

End Sub 
Private Sub datPrimaryRS_Error(ByVal ErrorNumber As Long, Description As String, ByVal Scode As Long, ByVal Source As String, ByVal HelpFile As String, ByVal HelpContext As Long, fCancelDisplay As Boolean)

'This is where you would put error handling code

'If you want to ignore errors, comment out the next line

'If you want to trap them, add code here to handle them

MsgBox "Data error event hit err:" \& Description

End Sub

Private Sub datPrimaryRS_MoveComplete(ByVal adReason As ADODB.EventReasonEnum, ByVal pError As

ADODB.Error, adStatus As ADODB.EventStatusEnum, ByVal pRecordset As ADODB.Recordset)

'This will display the current record position for this recordset

datPrimaryRS.Caption = "Record: " \& CStr(datPrimaryRS.Recordset.AbsolutePosition)

End Sub

Private Sub datPrimaryRS_WillChangeRecord(ByVal adReason As ADODB.EventReasonEnum, ByVal cRecords As Long, adStatus As ADODB.EventStatusEnum, ByVal pRecordset As ADODB.Recordset)

'This is where you put validation code

'This event gets called when the following actions occur

Dim bCancel As Boolean

Select Case adReason

Case adRsnAddNew

Case adRsnClose

Case adRsnDelete

Case adRsnFirstChange

Case adRsnMove

Case adRsnRequery

Case adRsnResynch

Case adRsnUndoAddNew

Case adRsnUndoDelete

Case adRsnUndoUpdate

Case adRsnUpdate

End Select

If $b$ Cancel Then adStatus $=$ adStatusCancel

End Sub

Private Sub cmdAdd_Click()

On Error GoTo AddErr

datPrimaryRS.Recordset.AddNew

Exit Sub

AddErr:

MsgBox Err.Description

End Sub

Private Sub cmdDelete_Click()

On Error GoTo DeleteErr

With datPrimaryRS.Recordset

.Delete

.MoveNext

If .EOF Then .MoveLast

End With

Exit Sub

DeleteErr:

MsgBox Err.Description 


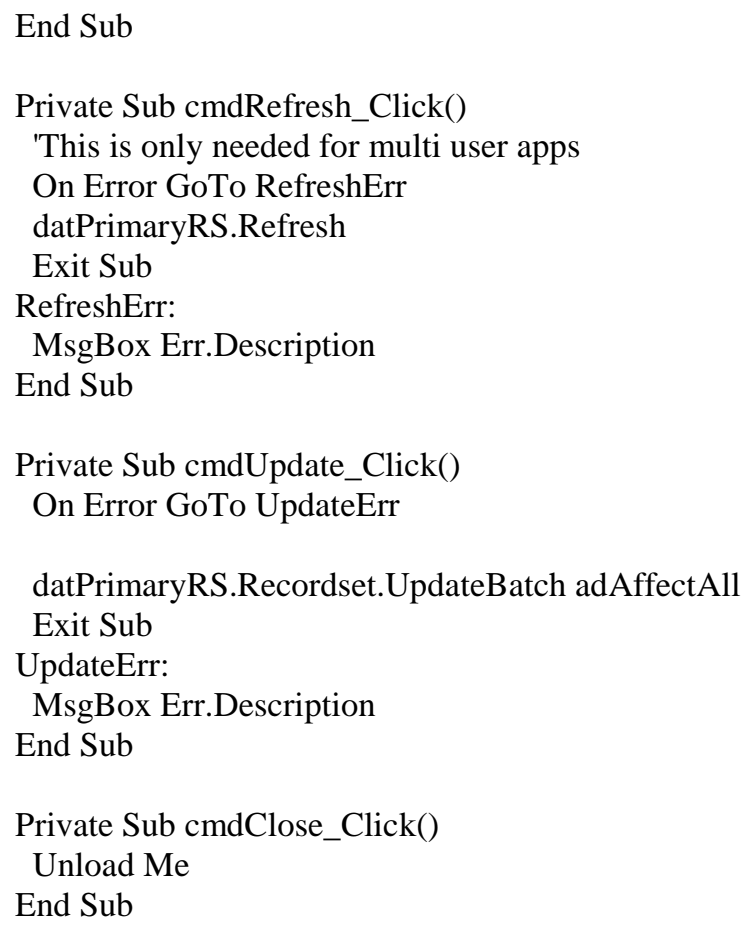

\subsection{Invalid Password Code:}

Private Sub Command1_Click()

If Text1.Text = "a" Then

Unload frmpassword

frmmain. Show 1

Else

Unload frmpassword

MsgBox ("Invalid password")

frmpassword. Show 1

Text1.Text $=$ ""

End If

End Sub

\subsection{Percent Dissociated Code:}

Private Sub Form_Unload(Cancel As Integer)

Screen.MousePointer $=$ vbDefault

End Sub

Private Sub datPrimaryRS_Error(ByVal ErrorNumber As Long, Description As String, ByVal Scode As Long, ByVal Source As String, ByVal HelpFile As String, ByVal HelpContext As Long, fCancelDisplay As Boolean)

'This is where you would put error handling code

'If you want to ignore errors, comment out the next line

'If you want to trap them, add code here to handle them

MsgBox "Data error event hit err:" \& Description

End Sub 
Private Sub datPrimaryRS_MoveComplete(ByVal adReason As ADODB.EventReasonEnum, ByVal pError As ADODB.Error, adStatus As ADODB.EventStatusEnum, ByVal pRecordset As ADODB.Recordset)

'This will display the current record position for this recordset

datPrimaryRS.Caption = "Record: " \& CStr(datPrimaryRS.Recordset.AbsolutePosition)

End Sub

Private Sub datPrimaryRS_WillChangeRecord(ByVal adReason As ADODB.EventReasonEnum, ByVal cRecords As Long, adStatus As ADODB.EventStatusEnum, ByVal pRecordset As ADODB.Recordset)

'This is where you put validation code

'This event gets called when the following actions occur

Dim bCancel As Boolean

Select Case adReason

Case adRsnAddNew

Case adRsnClose

Case adRsnDelete

Case adRsnFirstChange

Case adRsnMove

Case adRsnRequery

Case adRsnResynch

Case adRsnUndoAddNew

Case adRsnUndoDelete

Case adRsnUndoUpdate

Case adRsnUpdate

End Select

If $b$ Cancel Then adStatus $=$ adStatusCancel

End Sub

Private Sub cmdAdd_Click()

On Error GoTo AddErr

datPrimaryRS.Recordset.AddNew

Exit Sub

AddErr:

MsgBox Err.Description

End Sub

Private Sub cmdDelete_Click()

On Error GoTo DeleteErr

With datPrimaryRS.Recordset

.Delete

.MoveNext

If .EOF Then .MoveLast

End With

Exit Sub

DeleteErr:

MsgBox Err.Description

End Sub

Private Sub cmdRefresh_Click()

'This is only needed for multi user apps

On Error GoTo RefreshErr

datPrimaryRS.Refresh

Exit Sub

RefreshErr: 


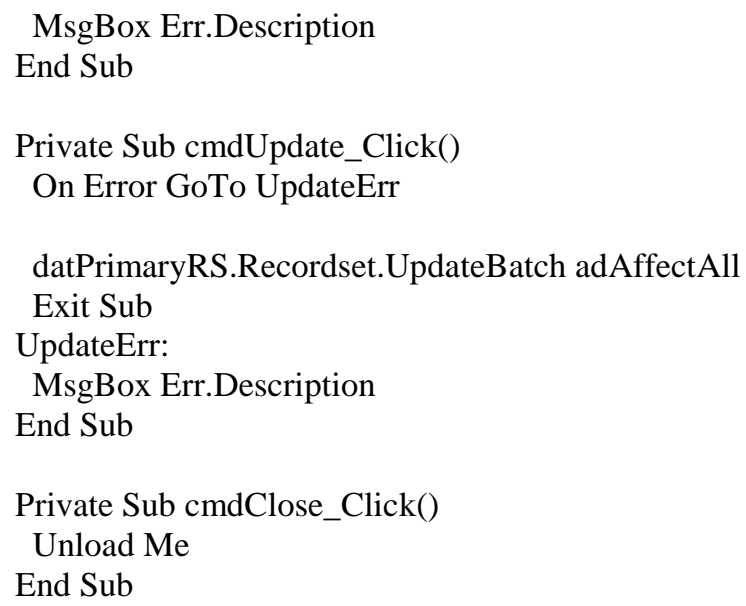

\subsection{Form Query on boiling point:}

Private optCheck As Boolean

Private Sub Option1_Click()

optCheck $=$ True

End Sub

Private Sub Option2_Click()

optCheck $=$ False

End Sub

Private Sub Text1_KeyPress(KeyAscii As Integer)

If KeyAscii = 13 Then

If Text1.Text $=" "$ Then

MsgBox "Please enter a value for Boiling Point"

Exit Sub

Else

If optCheck Then

adoQmw.RecordSource $=$ "select $*$ from materials where boiling_point is not null and boiling_point $>$ " \&

Text1.Text \& " order by boiling_point" adoQmw.Refresh

Else

adoQmw.RecordSource $=$ "select $*$ from materials where boiling_point is not null and boiling_point $<$ " \&

Text1.Text \& " order by boiling_point" adoQmw.Refresh

End If

End If

End If

End Sub

\subsection{Query on Classification form code:}

Private optCheck As Boolean 
Private Sub Option1_Click()

optCheck $=$ True

End Sub

Private Sub Option2_Click()

optCheck $=$ False

End Sub

Private Sub Combo1_click()

adoQmw.RecordSource $=$ "select $*$ from materials where classification $=$ "' \& Combo1.Text \& "'"

adoQmw.Refresh

End Sub

Private Sub Form_Load()

Dim dbcs As Database

Dim rscs As Recordset

Dim i

Set dbcs = DBEngine. Workspaces(0).OpenDatabase("c:lvdemoldb2.mdb")

Set rscs $=$ dbcs.OpenRecordset("select distinct classification from materials")

Do Until rscs.EOF

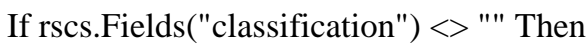

Combo1.AddItem rscs!classification

End If

rscs.MoveNext

Loop

rscs.Close

dbcs.Close

End Sub

\subsection{Query on Melting point code}

Private optCheck As Boolean

Private Sub Option1_Click()

optCheck $=$ True

End Sub

Private Sub Option2_Click()

optCheck $=$ False

End Sub

Private Sub Text1_KeyPress(KeyAscii As Integer)

If KeyAscii = 13 Then

If Text1.Text $=$ "" Then

MsgBox "Please enter a value for Melting Point"

Exit Sub

Else

If optCheck Then

adoQmw.RecordSource $=$ "select $*$ from materials where melting_point is not null and melting_point $>" \&$

Text1.Text \& " order by melting_point" adoQmw.Refresh

Else

adoQmw.RecordSource $=$ "select $*$ from materials where melting_point is not null and melting_point $<" \&$

Text1.Text \& " order by melting_point" 


adoQmw.Refresh
End If
End If
End If
End Sub

\subsection{Query on Molecular Weight code:}

Private optCheck As Boolean

Private Sub Option1_Click()

optCheck $=$ True

End Sub

Private Sub Option2_Click()

optCheck $=$ False

End Sub

Private Sub Text1_KeyPress(KeyAscii As Integer)

If KeyAscii $=13$ Then

If Text1.Text $=$ "" Then

MsgBox "Please enter a value for Molecular weight"

Exit Sub

Else

If optCheck Then

adoQmw.RecordSource $=$ "select $*$ from materials where molecular_wt is not null and molecular_wt $>$ " \&

Text1.Text \& " order by molecular_wt" adoQmw.Refresh

Else

adoQmw.RecordSource $=$ "select $*$ from materials where molecular_wt is not null and molecular_wt $<" \&$

Text1.Text $\&$ " order by molecular_wt" adoQmw.Refresh

End If

End If

End If

End Sub

\subsection{Query on VP Code}

Private optCheck As Boolean

Private Sub Option1_Click()

optCheck $=$ True

End Sub

Private Sub Option2_Click()

optCheck $=$ False

End Sub

Private Sub Text1_KeyPress(KeyAscii As Integer) 
If KeyAscii = 13 Then

If Text1.Text $=" "$ Then

MsgBox "Please enter a value for Vapor Pressure"

Exit Sub

Else

If optCheck Then

adoQmw.RecordSource $=$ "select $*$ from materials where vp is not null and vp $>$ " \& Text1.Text \& " order by $\mathrm{vp}^{\prime \prime}$ adoQmw.Refresh

Else

adoQmw.RecordSource $=$ "select $*$ from materials where vp is not null and vp $<" \&$ Text1.Text $\&$ " order by

vp"

adoQmw.Refresh

End If

End If

End If

End Sub

\subsection{Reactivity/Instability form code:}

Private Sub Form_Unload(Cancel As Integer)

Screen.MousePointer $=$ vbDefault

End Sub

Private Sub datPrimaryRS_Error(ByVal ErrorNumber As Long, Description As String, ByVal Scode As Long, ByVal Source As String, ByVal HelpFile As String, ByVal HelpContext As Long, fCancelDisplay As Boolean)

'This is where you would put error handling code

'If you want to ignore errors, comment out the next line

'If you want to trap them, add code here to handle them

MsgBox "Data error event hit err:" \& Description

End Sub

Private Sub datPrimaryRS_MoveComplete(ByVal adReason As ADODB.EventReasonEnum, ByVal pError As ADODB.Error, adStatus As ADODB.EventStatusEnum, ByVal pRecordset As ADODB.Recordset)

'This will display the current record position for this recordset

datPrimaryRS.Caption = "Record: " \& CStr(datPrimaryRS.Recordset.AbsolutePosition)

End Sub

Private Sub datPrimaryRS_WillChangeRecord(ByVal adReason As ADODB.EventReasonEnum, ByVal cRecords As Long, adStatus As ADODB.EventStatusEnum, ByVal pRecordset As ADODB.Recordset)

'This is where you put validation code

'This event gets called when the following actions occur

Dim bCancel As Boolean

Select Case adReason

Case adRsnAddNew

Case adRsnClose

Case adRsnDelete

Case adRsnFirstChange

Case adRsnMove

Case adRsnRequery

Case adRsnResynch

Case adRsnUndoAddNew

Case adRsnUndoDelete 


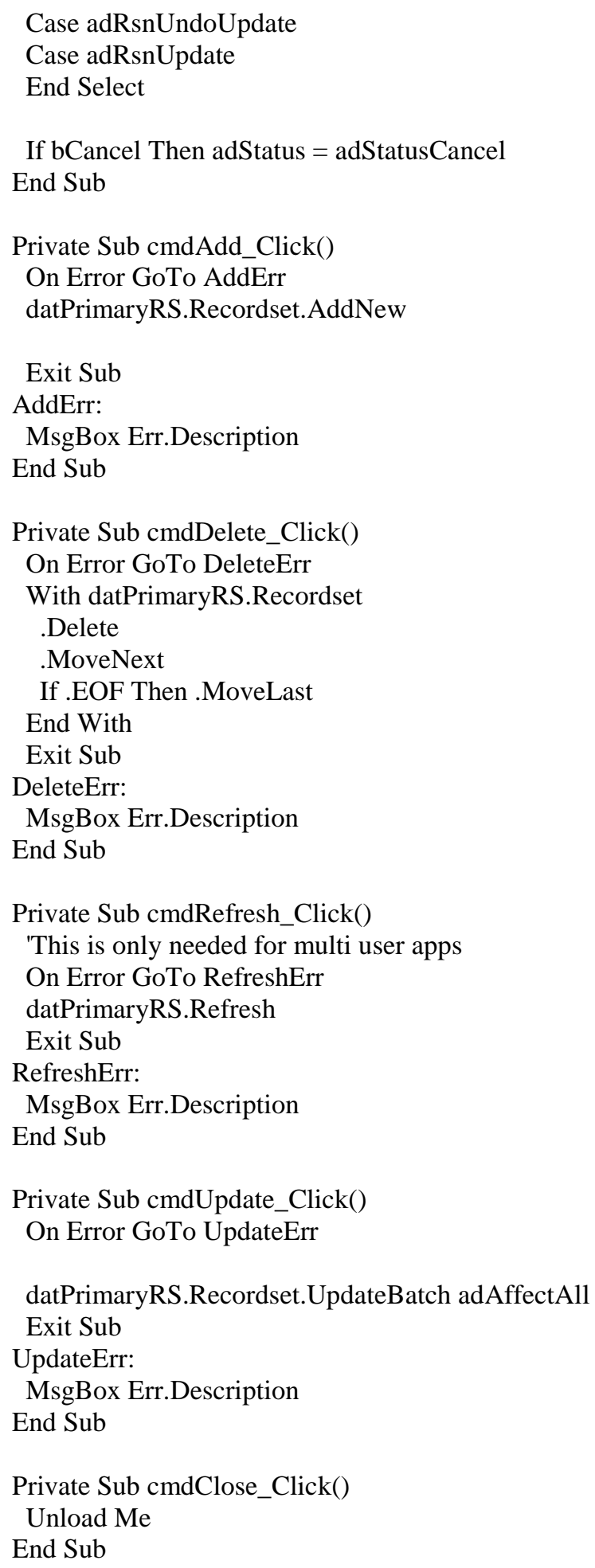

\subsection{Search By chemical Name Form code:}

Option Explicit

Private dbcs As Database

Private rscs As Recordset 


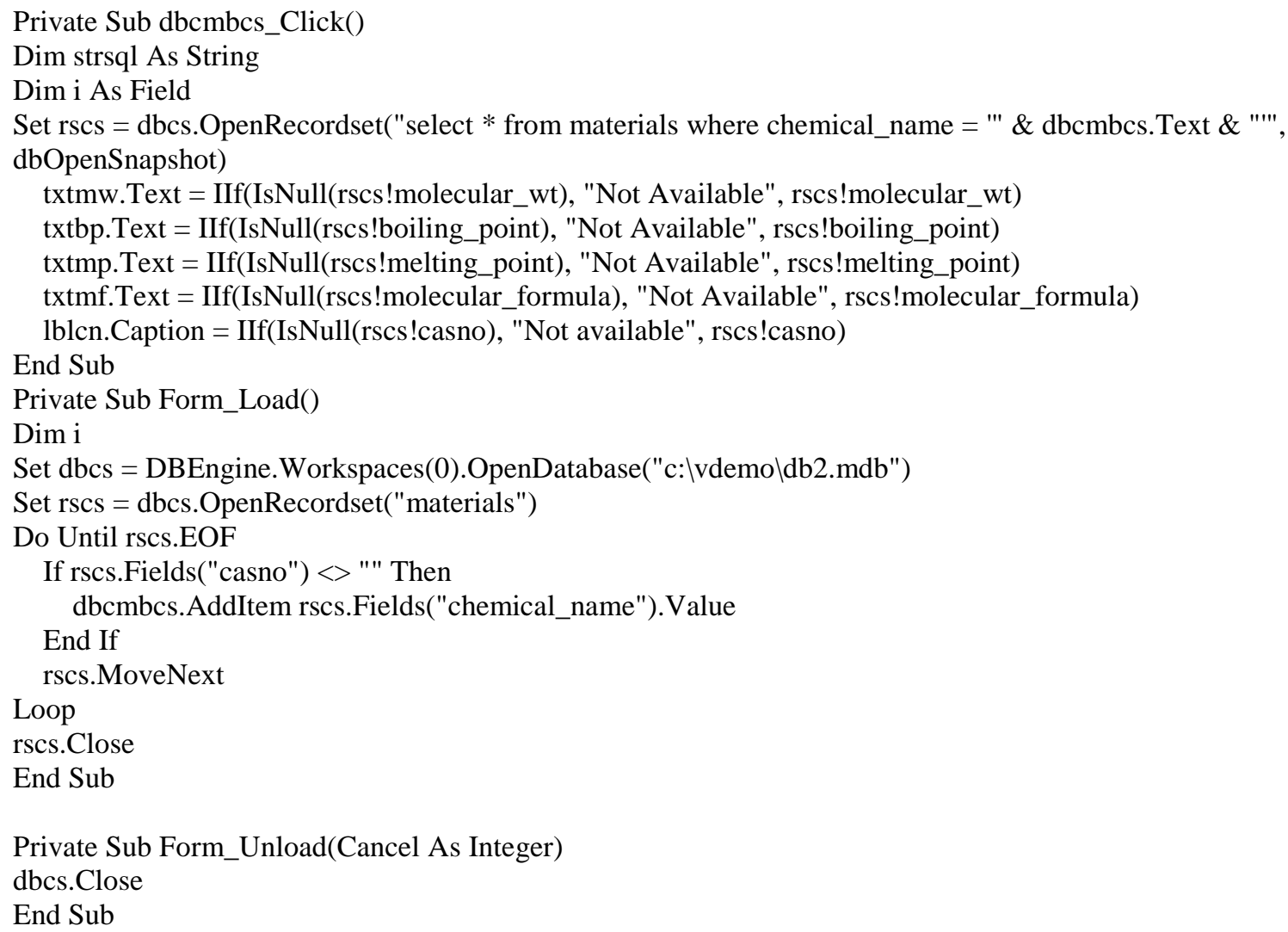

\subsection{Search By CAS number code:}

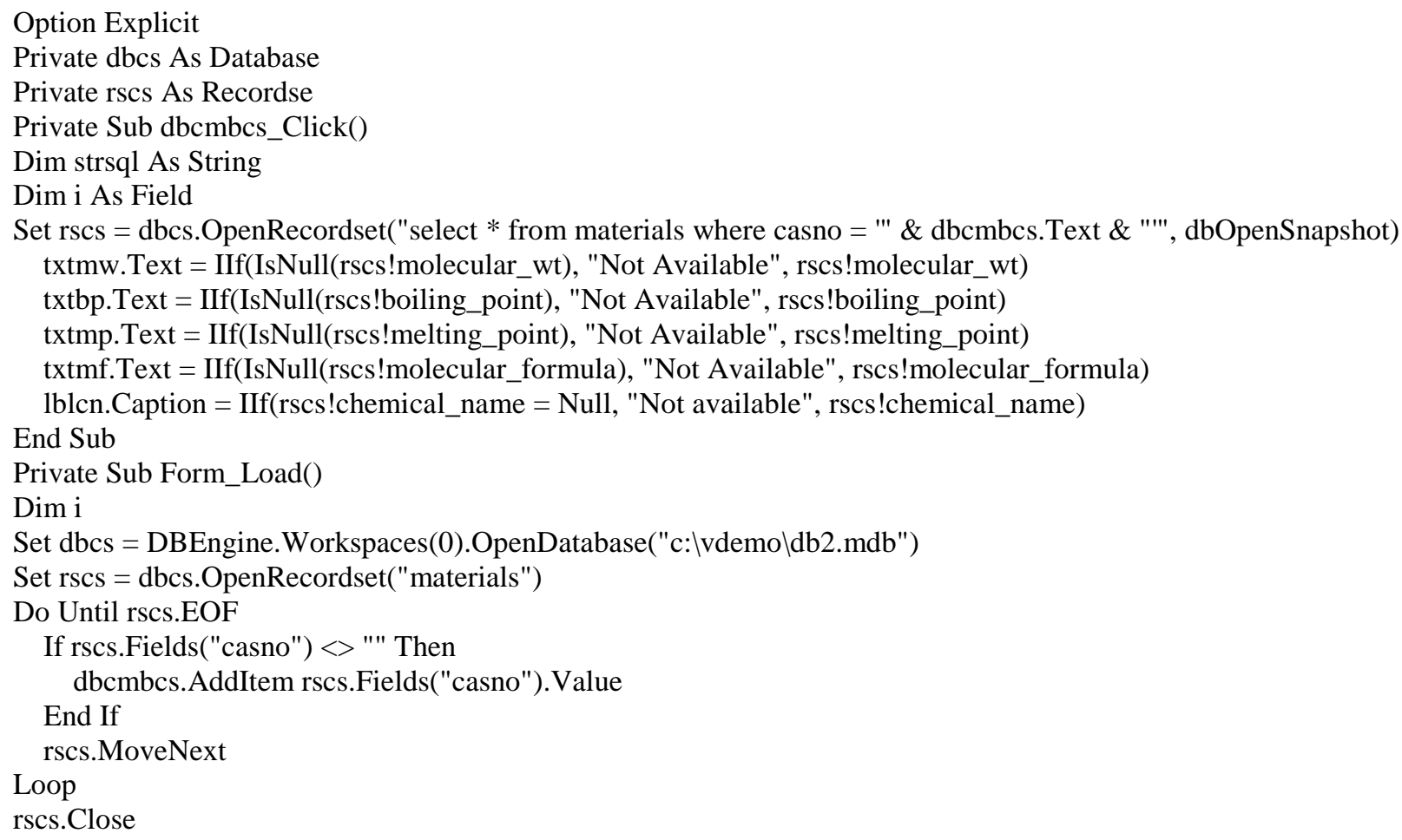


End Sub

Private Sub Form_Unload(Cancel As Integer)

dbcs.Close

End Sub

\subsection{Search by Molecular Formula Code}

Option Explicit

Private dbcs As Database

Private rscs As Recordset

Private Sub dbcmbcs_Click()

Dim strsql As String

Dim i As Field

Set rscs = dbcs.OpenRecordset("select $*$ from materials where molecular_formula = "' \& dbcmbcs.Text $\&$ "'", dbOpenSnapshot)

txtmw.Text = IIf(IsNull(rscs!molecular_wt), "Not Available", rscs!molecular_wt)

txtbp.Text = IIf(IsNull(rscs!boiling_point), "Not Available", rscs!boiling_point)

txtmp.Text = IIf(IsNull(rscs!melting_point), "Not Available", rscs!melting_point)

lblcn.Caption $=$ IIf(rscs!chemical_name = Null, "Not available", rscs!chemical_name)

End Sub

Private Sub Form_Load()

Dim i

Set dbcs = DBEngine. Workspaces(0).OpenDatabase("c:lvdemoldb2.mdb")

Set rscs = dbcs.OpenRecordset("materials")

Do Until rscs.EOF

If rscs.Fields("molecular_formula") <> "" Then dbcmbcs.AddItem rscs.Fields("molecular_formula").Value

End If

rscs.MoveNext

Loop

rscs.Close

End Sub

Private Sub Form_Unload(Cancel As Integer)

dbcs.Close

End Sub

\subsection{Start up form code:}

Private Sub Command1_Click()

frmpassword. Show 1

End Sub

Private Sub Command2_Click()

End

End Sub 


\subsection{Terrestrial Fate form code:}

Private Sub Form_Unload(Cancel As Integer)

Screen.MousePointer $=$ vbDefault

End Sub

Private Sub datPrimaryRS_Error(ByVal ErrorNumber As Long, Description As String, ByVal Scode As Long, ByVal Source As String, ByVal HelpFile As String, ByVal HelpContext As Long, fCancelDisplay As Boolean)

'This is where you would put error handling code

'If you want to ignore errors, comment out the next line

'If you want to trap them, add code here to handle them

MsgBox "Data error event hit err:" \& Description

End Sub

Private Sub datPrimaryRS_MoveComplete(ByVal adReason As ADODB.EventReasonEnum, ByVal pError As ADODB.Error, adStatus As ADODB.EventStatusEnum, ByVal pRecordset As ADODB.Recordset)

'This will display the current record position for this recordset

datPrimaryRS.Caption = "Record: " \& CStr(datPrimaryRS.Recordset.AbsolutePosition)

End Sub

Private Sub datPrimaryRS_WillChangeRecord(ByVal adReason As ADODB.EventReasonEnum, ByVal cRecords As Long, adStatus As ADODB.EventStatusEnum, ByVal pRecordset As ADODB.Recordset)

'This is where you put validation code

'This event gets called when the following actions occur

Dim bCancel As Boolean

Select Case adReason

Case adRsnAddNew

Case adRsnClose

Case adRsnDelete

Case adRsnFirstChange

Case adRsnMove

Case adRsnRequery

Case adRsnResynch

Case adRsnUndoAddNew

Case adRsnUndoDelete

Case adRsnUndoUpdate

Case adRsnUpdate

End Select

If $b$ Cancel Then adStatus $=$ adStatusCancel

End Sub

Private Sub cmdAdd_Click()

On Error GoTo AddErr

datPrimaryRS.Recordset.AddNew

Exit Sub

AddErr:

MsgBox Err.Description

End Sub

Private Sub cmdDelete_Click()

On Error GoTo DeleteErr

With datPrimaryRS.Recordset

.Delete 


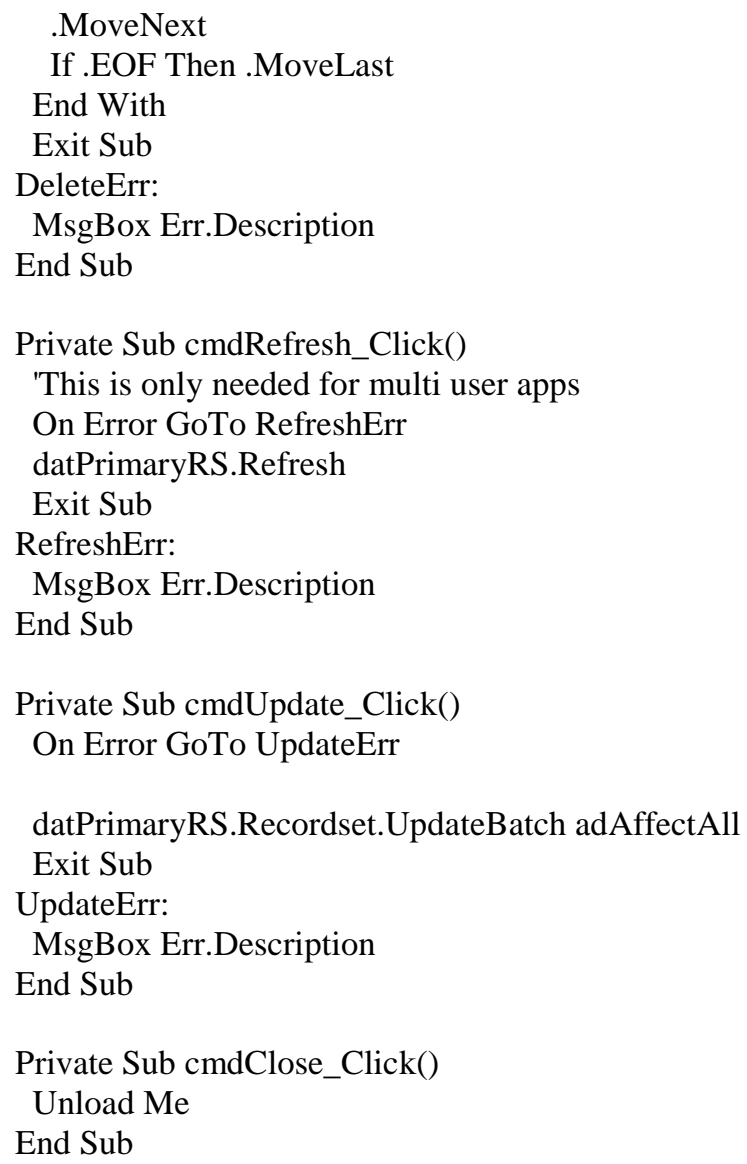

\subsection{Water Solubility Form code:}

Private Sub Form_Unload(Cancel As Integer)

Screen.MousePointer $=$ vbDefault

End Sub

Private Sub datPrimaryRS_Error(ByVal ErrorNumber As Long, Description As String, ByVal Scode As Long, ByVal Source As String, ByVal HelpFile As String, ByVal HelpContext As Long, fCancelDisplay As Boolean)

'This is where you would put error handling code

'If you want to ignore errors, comment out the next line

'If you want to trap them, add code here to handle them

MsgBox "Data error event hit err:" \& Description

End Sub

Private Sub datPrimaryRS_MoveComplete(ByVal adReason As ADODB.EventReasonEnum, ByVal pError As ADODB.Error, adStatus As ADODB.EventStatusEnum, ByVal pRecordset As ADODB.Recordset)

'This will display the current record position for this recordset

datPrimaryRS.Caption = "Record: " \& CStr(datPrimaryRS.Recordset.AbsolutePosition)

End Sub

Private Sub datPrimaryRS_WillChangeRecord(ByVal adReason As ADODB.EventReasonEnum, ByVal cRecords As Long, adStatus As ADODB.EventStatusEnum, ByVal pRecordset As ADODB.Recordset)

'This is where you put validation code

'This event gets called when the following actions occur

Dim bCancel As Boolean 
Select Case adReason

Case adRsnAddNew

Case adRsnClose

Case adRsnDelete

Case adRsnFirstChange

Case adRsnMove

Case adRsnRequery

Case adRsnResynch

Case adRsnUndoAddNew

Case adRsnUndoDelete

Case adRsnUndoUpdate

Case adRsnUpdate

End Select

If bCancel Then adStatus $=$ adStatusCancel

End Sub

Private Sub cmdAdd_Click()

On Error GoTo AddErr

datPrimaryRS.Recordset.AddNew

Exit Sub

AddErr:

MsgBox Err.Description

End Sub

Private Sub cmdDelete_Click()

On Error GoTo DeleteErr

With datPrimaryRS.Recordset

.Delete

.MoveNext

If .EOF Then .MoveLast

End With

Exit Sub

DeleteErr:

MsgBox Err.Description

End Sub

Private Sub cmdRefresh_Click()

'This is only needed for multi user apps

On Error GoTo RefreshErr

datPrimaryRS.Refresh

Exit Sub

RefreshErr:

MsgBox Err.Description

End Sub

Private Sub cmdUpdate_Click()

On Error GoTo UpdateErr

datPrimaryRS.Recordset.UpdateBatch adAffectAll

Exit Sub

UpdateErr:

MsgBox Err.Description

End Sub 
Private Sub cmdClose_Click()

Unload Me

End Sub

\subsection{Worker Exposure Criteria (acute-CEIL) CODE;}

Private Sub Form_Unload(Cancel As Integer)

Screen.MousePointer $=$ vbDefault

End Sub

Private Sub datPrimaryRS_Error(ByVal ErrorNumber As Long, Description As String, ByVal Scode As Long, ByVal Source As String, ByVal HelpFile As String, ByVal HelpContext As Long, fCancelDisplay As Boolean)

'This is where you would put error handling code

'If you want to ignore errors, comment out the next line

'If you want to trap them, add code here to handle them

MsgBox "Data error event hit err:" \& Description

End Sub

Private Sub datPrimaryRS_MoveComplete(ByVal adReason As ADODB.EventReasonEnum, ByVal pError As

ADODB.Error, adStatus As ADODB.EventStatusEnum, ByVal pRecordset As ADODB.Recordset)

'This will display the current record position for this recordset

datPrimaryRS.Caption = "Record: " \& CStr(datPrimaryRS.Recordset.AbsolutePosition)

End Sub

Private Sub datPrimaryRS_WillChangeRecord(ByVal adReason As ADODB.EventReasonEnum, ByVal cRecords As Long, adStatus As ADODB.EventStatusEnum, ByVal pRecordset As ADODB.Recordset)

'This is where you put validation code

'This event gets called when the following actions occur

Dim bCancel As Boolean

Select Case adReason

Case adRsnAddNew

Case adRsnClose

Case adRsnDelete

Case adRsnFirstChange

Case adRsnMove

Case adRsnRequery

Case adRsnResynch

Case adRsnUndoAddNew

Case adRsnUndoDelete

Case adRsnUndoUpdate

Case adRsnUpdate

End Select

If bCancel Then adStatus $=$ adStatusCancel

End Sub

Private Sub cmdAdd_Click()

On Error GoTo AddErr

datPrimaryRS.Recordset.AddNew

Exit Sub

AddErr:

MsgBox Err.Description 


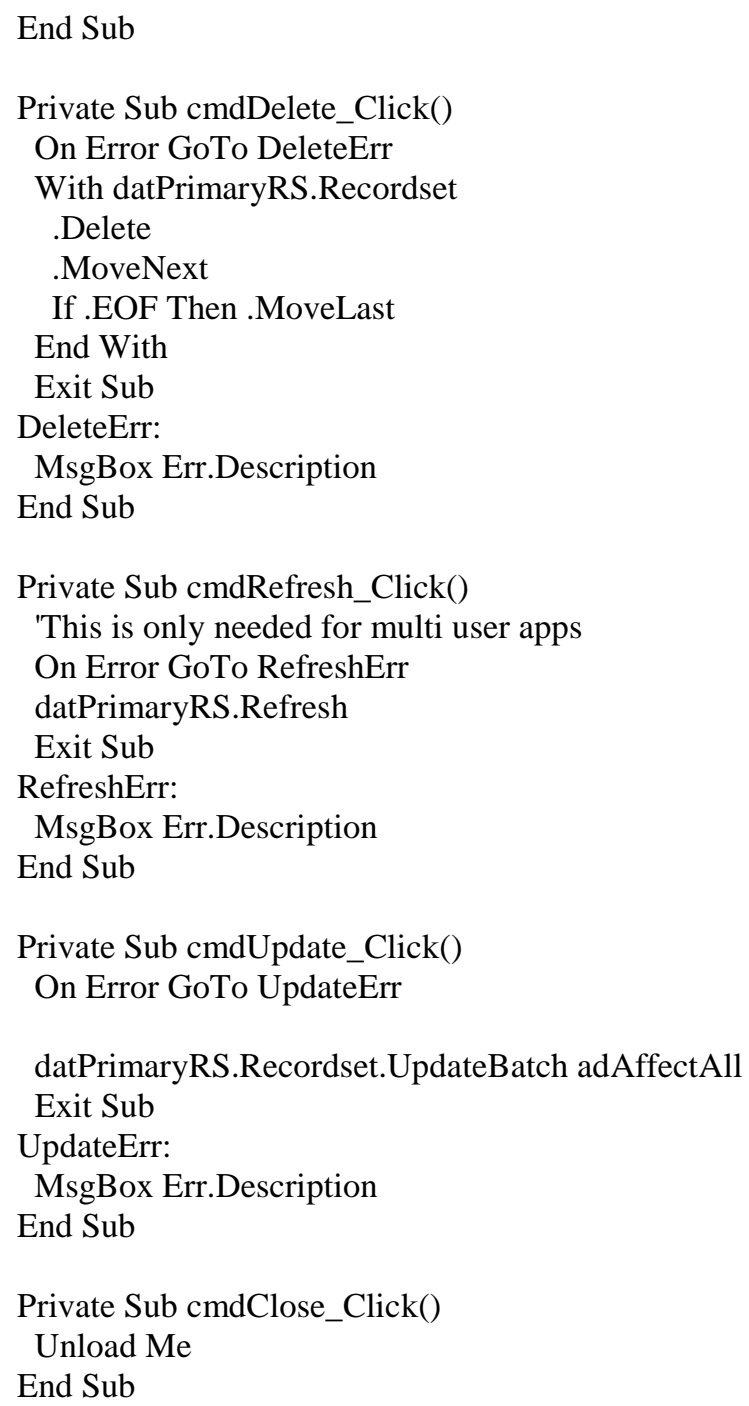

\subsection{Worker Exposure Criteria (acute-STEL) code}

Private Sub Form_Unload(Cancel As Integer)

Screen.MousePointer $=$ vbDefault

End Sub

Private Sub datPrimaryRS_Error(ByVal ErrorNumber As Long, Description As String, ByVal Scode As Long, ByVal Source As String, ByVal HelpFile As String, ByVal HelpContext As Long, fCancelDisplay As Boolean)

'This is where you would put error handling code

'If you want to ignore errors, comment out the next line

'If you want to trap them, add code here to handle them

MsgBox "Data error event hit err:" \& Description

End Sub

Private Sub datPrimaryRS_MoveComplete(ByVal adReason As ADODB.EventReasonEnum, ByVal pError As ADODB.Error, adStatus As ADODB.EventStatusEnum, ByVal pRecordset As ADODB.Recordset)

'This will display the current record position for this recordset

datPrimaryRS.Caption = "Record: " \& CStr(datPrimaryRS.Recordset.AbsolutePosition) 


\section{End Sub}

Private Sub datPrimaryRS_WillChangeRecord(ByVal adReason As ADODB.EventReasonEnum, ByVal cRecords As Long, adStatus As ADODB.EventStatusEnum, ByVal pRecordset As ADODB.Recordset)

'This is where you put validation code

'This event gets called when the following actions occur

Dim bCancel As Boolean

Select Case adReason

Case adRsnAddNew

Case adRsnClose

Case adRsnDelete

Case adRsnFirstChange

Case adRsnMove

Case adRsnRequery

Case adRsnResynch

Case adRsnUndoAddNew

Case adRsnUndoDelete

Case adRsnUndoUpdate

Case adRsnUpdate

End Select

If bCancel Then adStatus $=$ adStatusCancel

End Sub

Private Sub cmdAdd_Click()

On Error GoTo AddErr

datPrimaryRS.Recordset.AddNew

Exit Sub

AddErr:

MsgBox Err.Description

End Sub

Private Sub cmdDelete_Click()

On Error GoTo DeleteErr

With datPrimaryRS.Recordset

.Delete

.MoveNext

If .EOF Then .MoveLast

End With

Exit Sub

DeleteErr:

MsgBox Err.Description

End Sub

Private Sub cmdRefresh_Click()

'This is only needed for multi user apps

On Error GoTo RefreshErr

datPrimaryRS.Refresh

Exit Sub

RefreshErr:

MsgBox Err.Description

End Sub

Private Sub cmdUpdate_Click() 
On Error GoTo UpdateErr

datPrimaryRS.Recordset.UpdateBatch adAffectAll

Exit Sub

UpdateErr:

MsgBox Err.Description

End Sub

Private Sub cmdClose_Click()

Unload Me

End Sub

\subsection{Worker Exposure Criteria (chronicTWA) code:}

Private Sub Form_Unload(Cancel As Integer)

Screen.MousePointer $=$ vbDefault

End Sub

Private Sub datPrimaryRS_Error(ByVal ErrorNumber As Long, Description As String, ByVal Scode As Long, ByVal Source As String, ByVal HelpFile As String, ByVal HelpContext As Long, fCancelDisplay As Boolean)

'This is where you would put error handling code

'If you want to ignore errors, comment out the next line

'If you want to trap them, add code here to handle them

MsgBox "Data error event hit err:" \& Description

End Sub

Private Sub datPrimaryRS_MoveComplete(ByVal adReason As ADODB.EventReasonEnum, ByVal pError As ADODB.Error, adStatus As ADODB.EventStatusEnum, ByVal pRecordset As ADODB.Recordset)

'This will display the current record position for this recordset

datPrimaryRS.Caption = "Record: " \& CStr(datPrimaryRS.Recordset.AbsolutePosition)

End Sub

Private Sub datPrimaryRS_WillChangeRecord(ByVal adReason As ADODB.EventReasonEnum, ByVal cRecords As Long, adStatus As ADODB.EventStatusEnum, ByVal pRecordset As ADODB.Recordset)

'This is where you put validation code

'This event gets called when the following actions occur

Dim bCancel As Boolean

Select Case adReason

Case adRsnAddNew

Case adRsnClose

Case adRsnDelete

Case adRsnFirstChange

Case adRsnMove

Case adRsnRequery

Case adRsnResynch

Case adRsnUndoAddNew

Case adRsnUndoDelete

Case adRsnUndoUpdate

Case adRsnUpdate

End Select

If $b$ Cancel Then adStatus $=$ adStatusCancel

End Sub 


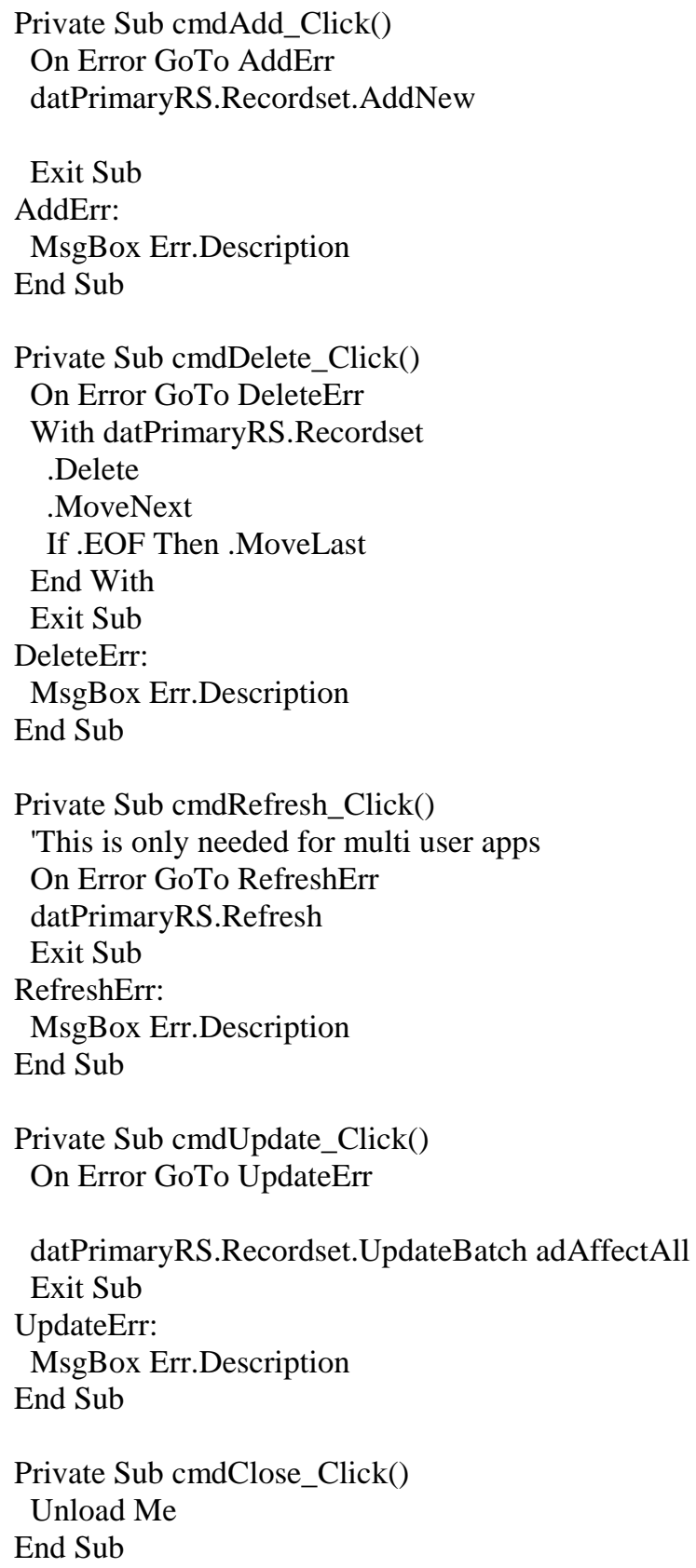




\section{APPENDIX 26 - RELATIONSHIPS DIAGRAM OF TABLES IN THE DATABASE}

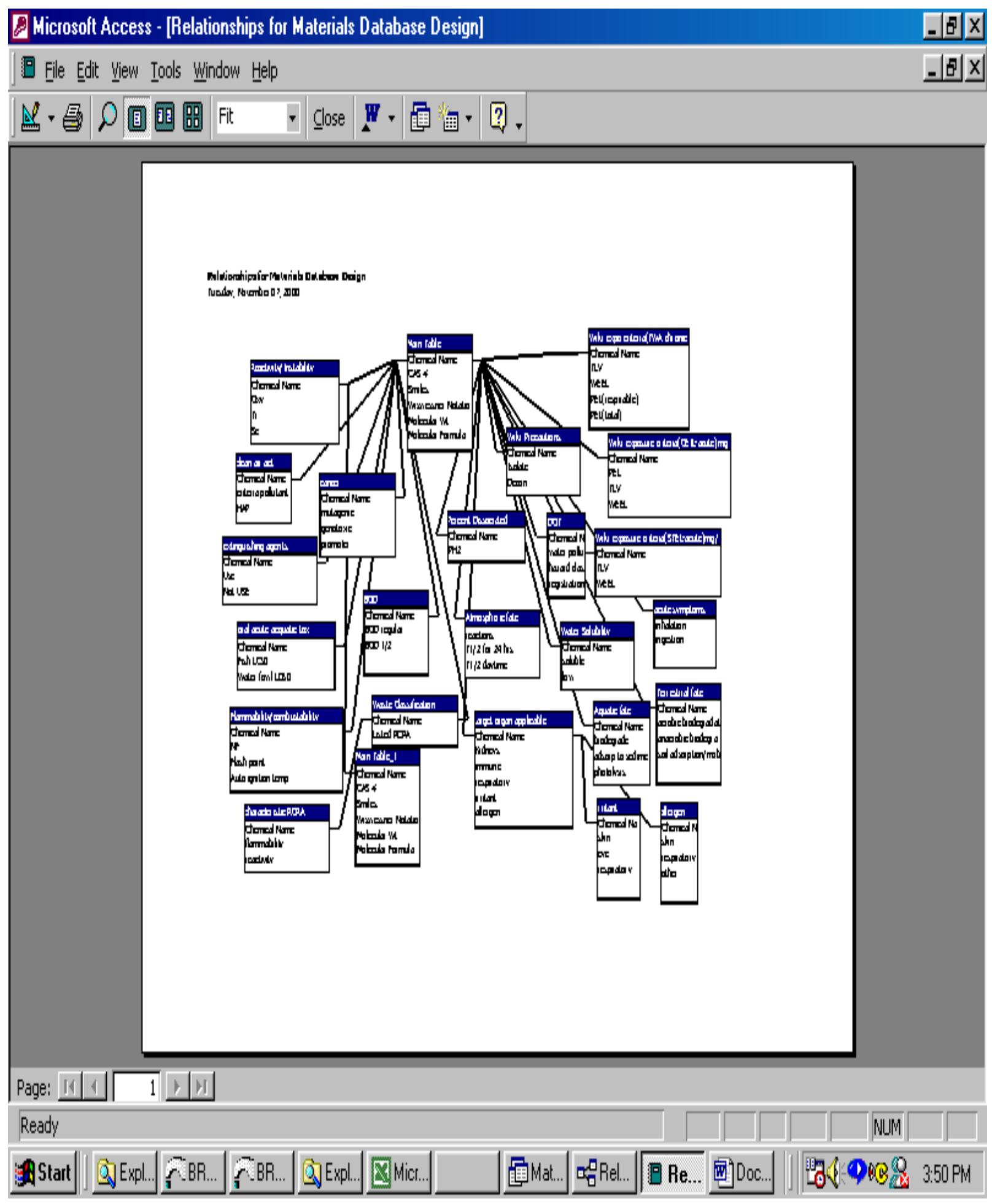

